\title{
Structure Analysis of the Pohlmeyer-Rehren Lie Algebra and \\ Adaptations of the Hall Algorithm to Non-Free Graded Lie Algebras
}

\section{Dissertation}

zur Erlangung des mathematisch-naturwissenschaftlichen Doktorgrades

"Doctor rerum naturalium"

der Georg-August-Universität Göttingen

im Promotionsprogramm "Mathematical Sciences (SMS)"

der Georg-August University School of Science (GAUSS)

vorgelegt von

Nils Bahne Hansen

aus Hamburg

Göttingen, 2020 


\title{
Betreuungsausschuss:
}

Prof. Dr. Dorothea Bahns,

Mathematisches Institut, Fakultät für Mathematik und Informatik

Karl-Henning Rehren, Institut für Theoretische Physik, Fakultät für Physik

\section{Mitglieder der Prüfungskommission:}

\author{
Referentin: \\ Prof. Dr. Dorothea Bahns, \\ Mathematisches Institut, \\ Fakultät für Mathematik und Informatik \\ Korreferent: \\ Prof. Dr. Karl-Henning Rehren, \\ Institut für Theoretische Physik, \\ Fakultät für Physik
}

\section{Weitere Mitglieder der Prüfungskommission:}

Prof. Dr. Thomas Schick,

Mathematisches Institut,

Fakultät für Mathematik und Informatik

Prof. Dr. Chenchang Zhu,

Mathematisches Institut,

Fakultät für Mathematik und Informatik

Prof. Dr. Laurent Bartholdi,

Mathematisches Institut,

Fakultät für Mathematik und Informatik

Prof. Dr. Max Wardetzky,

Institut für Numerische und Angewandte Mathematik, Fakultät für Mathematik und Informatik

Tag der mündlichen Prüfung: 18. 11. 2020 
Diese Dissertation ist gewidmet meiner Familie. Ich bin dankbar für ihre Unterstützung, ihre Geduld und ihre Liebe, ohne die dies nicht möglich gewesen wäre.

Meiner Betreuerin Frau Bahns danke ich für ihre Unterstützung dieses Vorhabens. 


\section{Contents}

$\begin{array}{ll}\text { Contents } & 4\end{array}$

\begin{tabular}{ll}
\hline Abstract & 7
\end{tabular}

\begin{tabular}{ll}
\hline Introduction & 9
\end{tabular}

\begin{tabular}{|lll}
\hline 1 & The Pohlmeyer-Rehren Lie Algebra & 13
\end{tabular}

1.1 The shuffle Hopf algebra . . . . . . . . . . . . . . . . . . . . . . . . . 13

1.2 The Eulerian idempotent $\ldots \ldots \ldots \ldots \ldots$. . . . . . . . . . . . . . . . . . . . . . . . . . . .

1.3 Lyndon words and Euler-Lyndon elements . . . . . . . . . . . . . . . . 20

1.4 The Pohlmeyer-Rehren Lie algebra . . . . . . . . . . . . . . . . . . . 24

1.5 Measures of growth of the Pohlmeyer-Rehren Lie algebra . . . . . . . . . . . 27

1.6 The spin basis . . . . . . . . . . . . . . . . . . . . . . . . . . . . . . 29

1.7 The Pohlmeyer-Rehren Lie algebra is not Kac-Moody . . . . . . . . . . . . . . 32

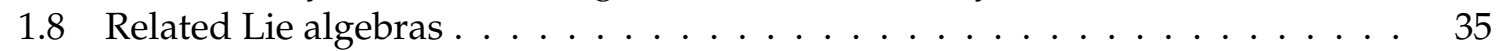

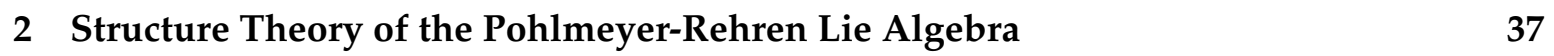

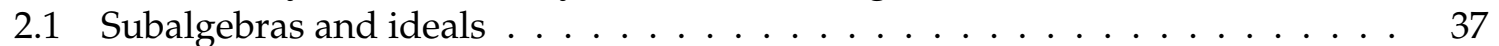

2.2 Structure of the subalgebra $g_{0} \ldots \ldots \ldots \ldots \ldots \ldots$

$2.2 .1 \mathfrak{g}_{0}$ is isomorphic to $\mathfrak{s v}(d, \mathbb{C}) \ldots \ldots \ldots \ldots \ldots$

2.2 .2 Cartan subalgebra of $g_{0} \quad \ldots \ldots \ldots \ldots$. . . . . . . . . . . . . 41

2.2.3 General outline of the root space decomposition . . . . . . . . . . . . 43

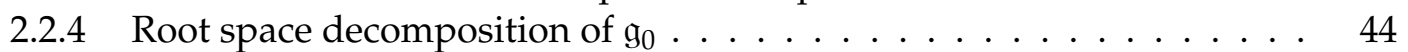

2.3 Weight space decomposition . . . . . . . . . . . . . . . . . . . . . . . . 49

$2.3 .1 \quad$ Overview of the general theory . . . . . . . . . . . . . . . . . 49

2.3.2 Application to the Pohlmeyer-Rehren Lie algebra . . . . . . . . . . 52

2.3.3 Explicit weight space decompositions of $\mathfrak{g}_{0}$ and $\mathfrak{g}_{1}$ for $d=3,4 \ldots 61$

2.4 Lie brackets of multiplets from the viewpoint of tensor and exterior products 65

3 Excursus: String Quantization and Leading Terms of Exceptional Elements of the $\begin{array}{ll}\text { Invariant Algebra } & 69\end{array}$

3.1 Pohlmeyer's approach . . . . . . . . . . . . . . . . . . . . . . . 69

3.1 .1 The Poisson algebra of invariant charges . . . . . . . . . . . . . 70

3.2 Poisson algebra of invariant charges in the shuffle Hopf language . . . . . . 72

3.2.1 Induced Poisson structure $i \ldots \ldots \ldots \ldots \ldots$. . . . . . . . . . 72

3.2 .2 Poisson algebra of invariant charges $\mathfrak{h} \ldots \ldots \ldots \ldots$. . . . . . . . 74

3.2 .3 The rest frame . . . . . . . . . . . . . . . . . . . . . 74 
3.2 .4 Generation of $\mathfrak{h}_{m}$ as a Poisson algebra . . . . . . . . . . . . . 75

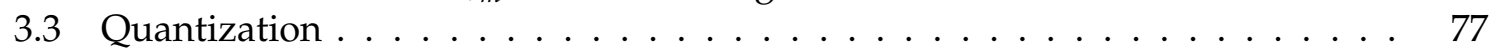

3.3.1 Pohlmeyer's original programme . . . . . . . . . . . . . . . . . 77

3.3.2 Meusburger-Rehren quantization . . . . . . . . . . . . . . . . . 77

3.3.3 Quantization of Poisson algebras . . . . . . . . . . . . . . . . . . . 79

3.3.4 Construction of the Meusburger-Rehren quantization . . . . . . . . . . 81

3.4 Leading terms of exceptional elements . . . . . . . . . . . . . . 83

\begin{tabular}{|lll}
4 & Computational Applications of Hall-Bases in Non-Free Graded Lie Algebras & 87
\end{tabular}

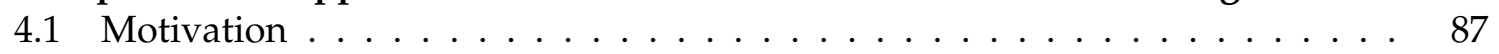

4.2 The Philip Hall algorithm for free Lie algebras . . . . . . . . . . . . . . . . . . . . 88

4.2 .1 Basic definitions $\ldots \ldots \ldots \ldots \ldots$

4.2 .2 Hall sets $\ldots \ldots \ldots \ldots \ldots$

4.2 .3 HaLL's algorithms . . . . . . . . . . . . . . . . . . . . . . . . 92

4.3 Extension to non-free Lie algebras $\ldots \ldots \ldots \ldots$. . . . . . . . . . . 95

4.3.1 Application to the Pohlmeyer-Rehren Lie algebra . . . . . . . . . . . . . . 104

4.4 Generation in order of an external, positive gradation . . . . . . . . . . . . 105

4.4.1 Application: Observations about the multiplet ${ }^{1} \mathfrak{g}_{1} \ldots \ldots$. . . . . . . 108

4.5 Admitting generators of degree zero $\ldots \ldots \ldots \ldots \ldots$

4.5 .1 Iterative extension to a basis . . . . . . . . . . . . . . . . . . . 110

$4.5 .2 \quad$ Iterative generation and subsequent extension to a basis $\ldots . . .115$

$\begin{array}{lll}5 & \text { Outlook } & 119\end{array}$

5.1 Interplay of the Hall algorithm and representation theory . . . . . . . . . . . . 119

5.2 Open questions regarding the Pohlmeyer-Rehren Lie algebra . . . . . . . . . . 120

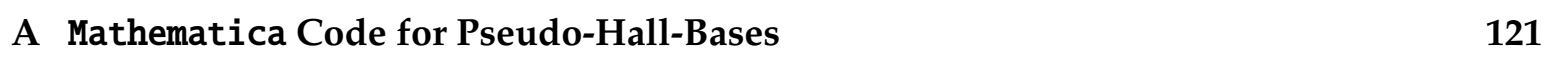

A.1 Orders . . . . . . . . . . . . . . . . . . . . . . . . . 121

A.2 Hall's original algorithm . . . . . . . . . . . . . . . . . . . . . . . . 122

A.3 Variants of the Hall algorithm . . . . . . . . . . . . . . . . . . . 124

B Mathematica Code for the Pohlmeyer-Rehren Lie algebra $\quad 131$

B.1 Shuffle product . . . . . . . . . . . . . . . . . . . . 131

B.2 Rewriting Euler elements in terms of Euler-Lyndon elements . . . . . . . . . . 133

B.3 Dimension formulas $\ldots \ldots \ldots \ldots \ldots \ldots \ldots \ldots \ldots$

B.4 Pohlmeyer-Rehren Lie bracket. . . . . . . . . . . . . . . . . . . . . . 137

B.5 Linear operators . . . . . . . . . . . . . . . . . . . . . . . . . . . . 138

B.6 Linear algebra tools $\ldots \ldots \ldots \ldots$. . . . . . . . . . . . . . . . . . . 139

C Calculations Regarding the Generation of Leading Terms of Exceptional Elements in $\hat{\mathfrak{b}} \quad 145$

D A Pseudo-Hall-Basis up to $l=4$ of $\mathfrak{g}$ for $d=3$

\begin{tabular}{ll}
\hline Bibliography & 201
\end{tabular} 



\begin{abstract}
The Pohlmeyer-Rehren Lie algebra $\mathfrak{g}$ is an infinite-dimensional $\mathbb{Z}$-graded Lie algebra that was discovered in the context of string quantization in $d$-dimensional spacetime by $\mathrm{K}$. Pohlmeyer and his collaborators and has more recently been reformulated in terms of the Euler-idempotents of the shuffle Hopf algebra.

This thesis is divided into two major parts. In the first part, the structure theory of $\mathfrak{g}$ is discussed. $\mathfrak{g}_{0}$, the stratum of degree zero, is isomorphic to the classical Lie algebra $\mathfrak{s}(d, \mathbb{C})$. Now, each stratum is considered as a $\mathfrak{g}_{0}$-module, and a formula for the number of irreducible $\mathfrak{g}_{0}$-modules of each highest weight that occur is given. It is also shown that $\mathfrak{g}$ is not a Kac-Moody algebra.

Based on computer-aided calculations, $\mathfrak{g}$ is conjectured to be generated by the strata of degrees 0 and 1, but not freely. In an effort to classify the relations, in the second part, the Philip Hall algorithm that iteratively lists (linear) basis elements of a Lie algebra $L(X)$ freely generated by a finite set of generators $X$ is modified. Any non-free finitely generated Lie algebra can be written as $L(X) / I$ with an ideal $I$ encoding the relations. Intended for cases where $I$ is not explicitly known, a variant of the algorithm iteratively lists a basis of $L(X) / I$ and a self-reduced basis of $I$. Further modifications that take advantage of restrictions enforced by a gradation on $L(X) / I$ are also given.
\end{abstract}





\section{Introduction}

\section{Strings}

A string is a one-dimensional object, modeled by a curve, open or closed, that moves through spacetime. As such, it is a generalization of a classical particle, which is conceived of as a zero-dimensional object, and many of the fundamental ideas can be transferred from the description of the particle.

As a particle moves through spacetime, it traces out a worldline, a curve which maps a parameter (usually the particle's proper time) to the particle's corresponding position in spacetime. The particle's dynamics can be described alternatively by its worldline being a solution to the particle's equations of motion, or equivalently, following HamiLton's principle, by the worldline being a stationary point of the action functional.

Analogously, the string moving through spacetime traces out a worldsheet, a two-dimensional surface which similarly is distinguished by the property that the action functional encapsulating the string's dynamics becomes stationary. A free string is described by the NamвU-Goто action, which is proportional to the worldsheet's surface area. We will only deal with closed strings, so their worldsheet can be described by a map

$$
x: S^{1} \times \mathbb{R} \rightarrow \mathbb{R}^{d},
$$

where the codomain is $d$-dimensional spacetime, equipped with the Minkowski metric, such that for each parameter $\tau$, the map $S^{1} \rightarrow \mathbb{R}^{d}, \sigma \mapsto x(\sigma, \tau)$ is a spacelike closed curve.

Since the parametrization $x$ of the worldsheet is thought of as having no physical significance other than fixing its image, the worldsheet, the worldsheet must be invariant under reparametrizations.

The usual approach to quantize the Nambu-Goto string that found its way into textbooks such as [Zwi04] and [BBS07] is to use methods of conformal field theory. Unfortunately it turns out that reparametrization invariance is broken when these methods are used. While invariance can be restored, it was first shown by C. Lovelace [Lov71] that this is only possible in spacetimes of a critical dimension of $d=26$ (or $d=10$ with supersymmetry) which leaves a number of dimensions that have to be compactified to explain that, apparently, we live in a spacetime with $d=4$.

\section{Pohlmeyer's approach to string quantization}

K. Pohlmeyer [Poh82] put forward a different approach to avoid these problems. In this approach, the string's worldsheet is described by a set of quantities that are defined in terms of functionals on the worldsheet that are invariant under reparametrization and are hence 
called the string's invariant charges or more concisely its invariants. The invariant charges form a $\mathbb{Z}$-graded Poisson algebra designated by $\mathfrak{h}$. The elements of $\mathfrak{h}$ encode the string's physical properties; Pohlmeyer and K.-H. Rehren proved [PR88, chapter III] that for a certain class of worldsheets, the worldsheet can be recovered from $\mathfrak{h}$ uniquely up to global translations in spacetime.

Originally, the Poisson algebra of invariant charges $\mathfrak{h}$ was constructed the following way. Using a LAx pair, a system of linear differential equations describing the string's dynamics is set up and solved. The power series expansion of the monodromy matrix of the differential equations leads to coefficients in the form of path-ordered integrals of products of $n$ factors of the so-called left and right movers (each designated by one of the dimensions of spacetime $\left.a_{i}\right)$, and are known as the tensors $\mathcal{R}_{a_{1} \ldots a_{n}}^{ \pm}$. Additionally, one can also perform a power series expansion on the logarithm of the monodromy matrix and obtain the so-called homogeneous tensors, in particular the truncated tensors $\mathcal{R}_{a_{1} \ldots a_{n}}^{ \pm t}$, or expand the trace of the logarithm of the monodromy matrix and obtain the homogeneous invariants. The term "invariant" is justified by the fact that they are in fact invariant under reparametrizations of the string's worldsheet. Due to the related construction of the aforementioned tensors and invariants, there are a number of relations between them; for instance the homogeneous invariants are called that way because they are homogeneous polynomials in the truncated tensors.

The homogeneous invariants generate the Poisson algebra of invariant charges $\mathfrak{h}$ where the multiplication is the regular multiplication of path-ordered integrals and the Poisson bracket is induced by the Poisson bracket on the left and right movers. A consequence of this is that the multiplication of the tensors $\mathcal{R}^{ \pm}$can be written using a shuffle product of indices,

$$
\mathcal{R}_{a_{1}, \ldots, a_{n}}^{ \pm} \mathcal{R}_{b_{1}, \ldots, b_{m}}^{ \pm}:=\mathcal{R}_{\left[\begin{array}{l} 
\pm a_{1}, \ldots, a_{n} \\
b_{1}, \ldots, b_{m}
\end{array}\right]}^{b^{\prime}} .
$$

Furthermore, the Poisson bracket of invariants can be understood as the derivative extension (with respect to the multiplication mentioned above) of a Lie bracket ${ }^{1}$ on the truncated tensors which turns the span of the truncated tensors into a Lie algebra called herein the Pohlmeyer-Rehren Lie algebra g.

However, not all elements of the Poisson algebra (called $\mathfrak{i}$ ) constructed this way are actually invariants. Instead, the Poisson algebra of invariants $\mathfrak{h}$ is a subalgebra of $\mathfrak{i}-$ to be more precise, the kernel of a derivation. In addition to the homogeneity degree, $\mathfrak{h}$ is equipped with a gradation such that multiplication is of degree +1 and the Poisson bracket is of degree 0 .

Rehren and Meusburger proposed an approach to quantization using the fact that $\mathfrak{h}$ is the kernel of a derivation. Based on the contained truncated tensors, they assign to each invariant of homogeneity degree two (called quadratic invariant) a quantized invariant, an element of the kernel of another derivation. The quantized invariants coincide with their classical counterparts except for a quantum correction of lower degree.

If the so-called quadratic generation hypothesis is true, the quadratic invariants generate the Poisson algebra of invariants, and we can therefore quantize non-quadratic invariants via their decomposition into quadratic invariants.

Because of its immediate physical significance, most work has been centered on the Poisson algebra of invariants $\mathfrak{h}$, while the Lie algebra $\mathfrak{g}$ was treated as a stepping stone in the

\footnotetext{
${ }^{1}$ Traditionally, this Lie bracket is called the modified Poisson bracket.
} 
construction, and while some interesting results on its structure were sought, found and used in the description of the structure of $\mathfrak{h}$, it was itself of rather secondary concern of research.

This dissertation however has $\mathfrak{g}$ and its structure as its primary focus. Not only is it of value to the question of quantization of the Poisson algebra of invariant charges of the Nambu-Goto string; as an infinite-dimensional graded Lie algebra it is interesting in its own right.

\section{Outline of this thesis}

In chapter 1. the basic terminology of the Pohlmeyer-Rehren Lie algebra is introduced. Instead of the original construction as an auxiliary Lie algebra used to construct the Poisson algebra of invariants of the Nambu-Goto string, a more concise construction due to D. BAHNs and J. Meinecke as the image of the Eulerian idempotent in the shuffle Hopf algebra is used.

It is proved that the Pohlmeyer-Rehren Lie algebra is not a Kac-Moody algebra, a large and well-researched class of infinite-dimensional Lie algebras that can be thought of as a generalization of finite-dimensional semisimple Lie algebras. In the end of the chapter, some related Lie algebras with the property that the sum of their Lie brackets is the PohlmeyerRehren Lie bracket are discussed.

Chapter 2 contains the main part of the structural analysis of the Pohlmeyer-Rehren Lie algebra given in this thesis. In particular, we use the fact that any $\mathbb{Z}$-graded Lie algebra (and any stratum of such a Lie algebra) is a module of its 0 -th stratum. It is proved that the 0 -th stratum $\mathfrak{g}_{0}$ is isomorphic to the special orthogonal Lie algebra $\mathfrak{s}(d, \mathbb{C})$.

Due to this fact, we can apply the method of weight space decomposition to $\mathfrak{g}$. After a brief overview of the general theory of weight spaces, the different strata of $\mathfrak{g}$ are decomposed into the weight spaces relative to $\mathfrak{g}_{0}$. By using counting arguments, a theorem (2.3.11) for the multiplicities of all occurring weights is given. This result is then applied to obtain a result (2.3.13) for the number of irreducible $\mathfrak{g}_{0}$-modules of a given highest weight.

Regarding a graded Lie algebra as a module of its 0 stratum does not take account of the Lie structure (except for the action of the stratum $\mathfrak{g}_{0}$ ). Lie brackets of entire irreducible $\mathfrak{g}_{0}$-modules (called multiplets) can be written as a direct sum of other multiplets. A discussion of this issue, which is known as the Clebsch-Gordan problem, concludes the chapter.

Chapter 3 provides a link to the original research that motivated this thesis. This chapter contains a brief introduction to POHLMEYER's approach to the description of a string's worldsheet by the Poisson algebra $\mathfrak{h}$ of its invariants and to the Meusburger-Rehren approach to string quantization by constructing a quantized algebra $\hat{\mathfrak{h}}$ of invariants as the kernel of a derivation, analogous to the fact that $\mathfrak{h}$ is the kernel of another derivation. The chapter ends with some insights 3.4 .3 and 3.4.2) into the generation of leading terms of exceptional elements.

Chapter 4 discusses applications of Hall bases to the problem of computing relations in non-free Lie algebras. Any Lie algebra can be viewed as the quotient of a free Lie algebra modulo an ideal containing all relations. A linear basis of a free Lie algebra is given by the Hall basis, which is a set of particular multiple Lie brackets of generators of the Lie algebra. The chapter now discusses modifications of an algorithm that lists elements of a Hall basis in order of their monomial degree to the non-free case, where it is used to find a generating set of the ideal of relations, and further adapts the algorithm in several steps to the specific situation of the Pohlmeyer-Rehren Lie algebra. 
Two appendices list Mathematica source code that was used for calculations in the context of this thesis. Appendix A provides implementations of the variants of the algorithm discussed in chapter 4 along with some examples.

Appendix $B$ lists code related to the Pohlmeyer-Rehren Lie algebra, among them implementations of the shuffle product, PoHLMEYER's algorithm for rewiting Euler words as EulerLyndon words and the Lie bracket on $\mathfrak{g}$. Furthermore, wrappers of in-built Mathematica linear algebra modules are given that are useful in the context of the thesis.

Appendix Cserves to document some partial results obtained in the pursuit of a proof of a conjecture (3.4.2) on the generation of leading terms of exceptional elements in the quantized algebra of invariants $\hat{\mathfrak{h}}$.

Finally, appendix $\mathrm{D}$ lists a basis of the Pohlmeyer-Rehren Lie algebra for spacetime dimension $d=3$ and a generating set of the ideal of relations up to stratum $l=4$, calculated by the algorithm PHallSecondGrad described in chapter 4 


\section{Chapter 1}

\section{The Pohlmeyer-Rehren Lie Algebra}

The Pohlmeyer-Rehren Lie algebra, the structure of which will be a main focus of this thesis, was discovered in the context of string quantization by K. Pohlmeyer and K.-H. Rehren (an account of the connection of this thesis to the work of PoHLMEyer and his collaborators can be found in chapter 3 .

Instead of following their research to establish a definition, a more straightforward definition based on the shuffle Hopf algebra that has been proved to be equivalent by D. BAHNs and J. MeINECKe [BM11] will be given. While the proofs used in the newer formulation generally follow the ideas from Pohlmeyer's and ReHren's work, they will frequently be cited here because they introduce the notation used in this thesis and are therefore easier to compare with it.

Before the Pohlmeyer-Rehren Lie algebra can be introduced, we need to establish some prerequisites. The discussion of standard facts and notions up to section 1.4 mainly follows MeInecke's account ([Mei09, chapters 3 and 4]) from her Diplom thesis, the first part of which is based on KasseL's book on quantum groups [Kas95, Chapters I through III], and the article [BM11]. Some notation used here is taken from DE GRAAF's book on algorithms for Lie algebras [dG00, section 6.1] to keep consistency with some similar concepts discussed in chapter 4 of this dissertation.

\subsection{The shuffle Hopf algebra}

Definition 1.1.1 (alphabet, letter, word, concatenation, free monoid, word algebra). Let $X$ be a set. We call $X$ the alphabet and its elements letters ${ }^{1}$ The $n$-tuples of letters are called words (of length $n$ ), and form the set $X^{n}$, where $n \in \mathbb{N}_{0}$. We call $X^{*}:=\bigcup_{n \in \mathbb{N}_{0}} X^{n}$ the set of words, noting that it includes the empty word, denoted by $1_{\emptyset}$ (which is the only element of $X^{0}$ ), and we define the concatenation of words,

$$
\begin{aligned}
X^{*} \times X^{*} & \rightarrow X^{*} \\
\left(x_{1} \ldots x_{n}\right) \frown\left(y_{1} \ldots y_{m}\right) & :=\left(x_{1} \ldots x_{n} y_{1} \ldots y_{m}\right),
\end{aligned}
$$

which equips $X^{*}$ with the structure of a monoid, called the free monoid on $X$. Its unit is the empty word $1_{\emptyset}$. Since the concatenation is associative, the parentheses delimiting the words

\footnotetext{
${ }^{1}$ In the literature, frequently only finite alphabets such as $X=\{0, \ldots d-1\}$ are used.
} 
and the concatenation symbol $\frown$ are often omitted. We denote repetitions of letters or other words by

$$
x^{n}:=\underbrace{x \ldots x}_{n}
$$

for $x \in X^{*}$ and $n \in \mathbb{N}_{0}$.

Let further $\mathbb{K}$ be a field of characteristic 0 , then the $\mathbb{K}$-vector space $\mathbb{K}\langle X\rangle$ is defined by the span of $X^{*}$ over $\mathbb{K}$. The concatenation can be extended bilinearly to an associative linear map $\mathbb{K}\langle X\rangle \times \mathbb{K}\langle X\rangle \rightarrow \mathbb{K}\langle X\rangle$, which makes $\left(\mathbb{K}\langle X\rangle, \frown, 1_{\emptyset}\right)$ an associative unital algebra, called the free associative unital algebra or the word algebra over $\mathbb{K}$. It is obviously graded by word length.

In addition to the concatenation, we can endow $\mathbb{K}\langle X\rangle$ with a further multiplication.

Definition 1.1.2 (shuffle permutations, shuffle product). $\quad 1$. Let $n, m \in \mathbb{N}_{0}$. We call the set

$$
S_{n, m}:=\left\{\rho \in S_{n+m} \mid \rho^{-1}(1)<\ldots<\rho^{-1}(n) \text { and } \rho^{-1}(n+1)<\ldots<\rho^{-1}(n+m)\right\}
$$

(where $S_{n}$ designates the set of permutations of $n$ elements) the set of shuffle permutations.

2. The shuffle product is the map $\mu: \mathbb{K}\langle X\rangle \otimes \mathbb{K}\langle X\rangle \rightarrow \mathbb{K}\langle X\rangle$ defined by extending the following map $\mu: X^{*} \times X^{*} \rightarrow \mathbb{K}\langle X\rangle$ bilinearly:

let $x=x_{1} \ldots x_{n} \in X^{n} \subset X^{*}, y=x_{n+1} \ldots x_{n+m} \in X^{m} \subset X^{*}$ be words. Then their shuffle product is defined as

$$
x \# y:=\left[\begin{array}{l}
x \\
y
\end{array}\right]:=\mu(x \otimes y):=\sum_{\pi \in S_{n, m}} x_{\pi(1)} \ldots x_{\pi(n+m)} \in \mathbb{K}\langle X\rangle_{n+m} .
$$

3. Define the unity $\eta: \mathbb{K} \rightarrow \mathbb{K}\langle X\rangle$ by

$$
\eta(k):=k \cdot 1_{\emptyset} \text { for all } k \in \mathbb{K} .
$$

Remark 1.1.3 (notation of the shuffle product). To understand the term "shuffle product", it is illustrative to imagine the words $x, y$ above as decks of playing cards, and a single shuffle permutation as one particular way to shuffle the two decks together; the shuffle product is the sum over all possible shuffles. In the mathematical literature the notations $x \# y$ and $x ш y$ (the latter from the cyrillic letter $m$, pronounced "sha") are frequently used for the shuffle product. The box notation $\begin{aligned} & x \\ & y\end{aligned}$ is due to Pohlmeyer, nicely conveys the image of two decks of cards being shuffled into each other from above and below, and is very well suited to situations where shuffles and concatenations are being used together, in particular when individual letters are being considered. It does, however, become cumbersome when shuffle products of many factors are used. Consider the examples of both notations

$$
\begin{aligned}
& e\left(\begin{array}{l|l|l}
x_{1} \ldots x_{i-1} & y_{j-1} \ldots y_{1} \\
x_{n} \ldots x_{i+1} & y_{j+1} \ldots y_{m}
\end{array}\right)=e\left(x_{1} \ldots x_{i-1} \# x_{n} \ldots x_{i+1} \frown y_{j-1} \ldots y_{1} \# y_{j+1} \ldots y_{m}\right), \\
& w_{1} \# \ldots \# w_{k}=\left[\begin{array}{c}
w_{1} \\
\vdots \\
w_{k}
\end{array} .\right.
\end{aligned}
$$

In the sequel, we will use both notations whereever they are more convenient. 
There is also a widely known recursive identity for the shuffle product that provides an equivalent, iterative, definition and is often convenient to use in proofs.

Lemma 1.1.4 (recursive formula for the shuffle product). Let $x=x_{1} \ldots x_{n} \in X^{n}$ and $y=y_{1} \ldots y_{m} \in X^{m}$ be words. Then:

$$
\left.\begin{array}{l}
x_{1} \ldots x_{n} \\
y_{1} \ldots y_{m}
\end{array}=\begin{array}{c}
x_{1} \ldots x_{n} \\
y_{1} \ldots y_{m-1}
\end{array} y_{m}+\begin{array}{l}
x_{1} \ldots x_{n-1} \\
y_{1} \ldots y_{m}
\end{array} x_{n}=x_{1} \begin{array}{l}
x_{2} \ldots x_{n} \\
y_{1} \ldots y_{m}
\end{array}\right]+y_{1} \begin{aligned}
& x_{1} \ldots x_{n} \\
& y_{2} \ldots y_{m}
\end{aligned} .
$$

Example 1.1.5. We calculate the shuffle product of the two words $a b$ and $a c$ in the alphabet $X=\{a, b, c\}$ :

$$
\left[\begin{array}{l}
a b \\
a c
\end{array} \mid=a b a c+2 a a b c+2 a a c b+a c a b .\right.
$$

For easy reference, we recapitulate some very basic definitions that will be used throughout this thesis.

Definition 1.1.6. (algebra, associative, commutative, abelian, unital, homo-/epi-/mono-/isomorphism, subalgebra, ideal, gradation, derivation (along a homomorphism))

1. Let $A$ be a vector space over a field $\mathbb{K}$ and let $\mu: A \times A \rightarrow A$ be a $\mathbb{K}$-bilinear map. We write $a \cdot a^{\prime}$ for $\mu\left(a, a^{\prime}\right)$. Then $(A, \mu)$ or $(A, \cdot)$ is called an algebra over $\mathbb{K}$.

2. An algebra $(A, \cdot)$ that satisfies $a \cdot b=b \cdot a$ for all $a, b \in A$ is called commutative or abelian.

3. An algebra $(A, \cdot)$ that satisfies $a \cdot(b \cdot c)=(a \cdot b) \cdot c$ for all $a, b, c \in A$ is called associative.

4. An algebra $(A, \cdot)$ over a field $\mathbb{K}$ equipped with a $\mathbb{K}$-linear map $\eta: \mathbb{K} \rightarrow A$ (called the unity) such that $\eta\left(1_{\mathbb{K}}\right) \cdot a=a=a \cdot \eta\left(1_{\mathbb{K}}\right)$ for all $a \in A$ is called unital.

5. Let $(A, \cdot)$ and $(B, *)$ be algebras over a field $\mathbb{K}$. A homomorphism / epimorphism / monomorphism / isomorphism of $\mathbb{K}$-vector spaces $\varphi: A \rightarrow B$ is called a homomorphism / epimorphism / monomorphism / isomorphism of (K-) algebras if

$$
\varphi\left(a \cdot a^{\prime}\right)=\varphi(a) * \varphi\left(a^{\prime}\right) \text { for all } a, a^{\prime} \in A .
$$

If an isomorphism between two algebras exists, they are called isomorphic.

6. Let $(A, \cdot)$ be an algebra and let $B$ be a vector subspace of $A$. If $B \cdot B \subset B$, we call $B$ a subalgebra of $A$, and if $A \cdot B \subset B$ and $B \cdot A \subset B$ (these conditions are the same if $A$ is a commutative or Lie algebra), we call $B$ an ideal of $A$. The kernel $\operatorname{ker} \varphi:=\varphi^{-1}(0) \subset A$ of any homomorphism of algebras $\varphi: A \rightarrow C$ is an ideal of $A$.

7. Let $(A, \cdot)$ be an algebra over a field $\mathbb{K}$ with ideal $B \subset A$. We can then define the set $A / B:=\{a+B \mid a \in A\}$, obtain the canonical projection $\pi: A \rightarrow A / B, a \mapsto a+B$ and define the multiplication

$$
\begin{aligned}
*: A / B \times A / B & \rightarrow A / B, \\
\left(a+B, a^{\prime}+B\right) & \mapsto a \cdot a^{\prime}+B .
\end{aligned}
$$

Now $(A / B, *)$ is an algebra over $\mathbb{K}$ called the quotient algebra $A$ mod $B$ and we have $B=\operatorname{ker} \pi$. 
8. Let $(I,+)$ be a semigroup. An algebra $A$ is called I-graded or equipped with a I-gradation if

$$
A=\bigoplus_{i \in I} A_{i}
$$

(direct sum of vector spaces; the summands $A_{i}$ are called strata) and

$$
A_{i} \cdot A_{i^{\prime}} \subset A_{i+i^{\prime}} \text { for all } i, i^{\prime} \in I \text {. }
$$

9. Let $(A, \cdot)$ be an algebra over a field $\mathbb{K}$. An endomorphism of $\mathbb{K}$-vector spaces $\partial: A \rightarrow A$ is called a derivation of $A$ if

$$
\partial\left(a \cdot a^{\prime}\right)=a \cdot \partial\left(a^{\prime}\right)+\partial(a) \cdot a^{\prime} \text { for all } a, a^{\prime} \in A .
$$

If $(B, *)$ is another algebra over $\mathbb{K}$ and $\varphi: A \rightarrow B$ is a homomorphism, then a homomorphism of $\mathbb{K}$-vector spaces $\partial: A \rightarrow B$ is called a derivation along the homomorphism $\varphi$ if

$$
\partial\left(a \cdot a^{\prime}\right)=\varphi(a) * \partial\left(a^{\prime}\right)+\partial(a) * \varphi\left(a^{\prime}\right) \text { for all } a, a^{\prime} \in A \text {. }
$$

Theorem 1.1.7. $(\mathbb{K}\langle X\rangle, \mu, \eta)$ is an associative, commutative, unital algebra, called the shuffle algebra $\operatorname{Sh}(X)$. It is graded by word length.

Proof. This immediately follows from the definition.

In addition to being an algebra, the shuffle algebra is equipped with more algebraic stucture. We quickly recapitulate $\mathrm{\Omega}^{2}$ some further commonly found definitions.

Definition 1.1.8. (coassociative counital coalgebra, bialgebra, convolution product, antipode, Hopf algebra, gradation, connectedness)

1. Let $H$ be a vector space over a field $\mathbb{K}$ and let $\Delta: H \rightarrow H \otimes H$ and $\epsilon: H \rightarrow \mathbb{K}$ be $\mathbb{K}$-linear maps with the property that the diagrams

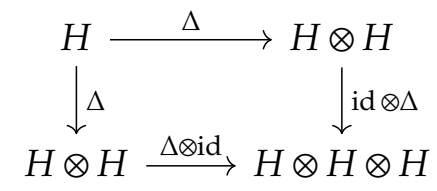

(coassociativity) and

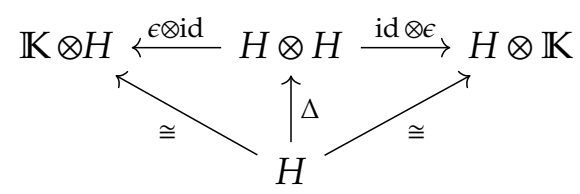

(counitality) commute. Then the triple $(H, \Delta, \epsilon)$ is called a coassociative counital coalgebra with the coproduct $\Delta$ and counit $\epsilon$.

2. Let $(H, \mu, \eta)$ be an associative unital algebra and $(H, \Delta, \epsilon)$ be a coassociative counital coalgebra. If $\Delta$ and $\epsilon$ are morphisms of algebras, then $(H, \mu, \eta, \Delta, \epsilon)$ is called a bialgebra.

\footnotetext{
${ }^{2}$ See [HGK10, Chapters 2 and 3] or [Kas95, Chapter III] for a comprehensive introduction to Hopf algebras.
} 
3. Let $(H, \mu, \eta, \Delta, \epsilon)$ be a bialgebra. The convolution product is defined by

$$
\begin{aligned}
*: \operatorname{End}_{\mathbb{K}}(H) \times \operatorname{End}_{\mathbb{K}}(H) & \rightarrow \operatorname{End}_{\mathbb{K}}(H), \\
f * g & :=\mu \circ(f \otimes g) \circ \Delta .
\end{aligned}
$$

It follows fom the definitions of (associative unital) algebra and (coassociative counital) coalgebra that $*$ is associative and $\eta \epsilon$ is its identity element.

4. Let $(H, \mu, \eta, \Delta, \epsilon)$ be a bialgebra. An endomorphism $S \in \operatorname{End}_{\mathbb{K}}(H)$ is called antipode if

$$
S * \mathrm{id}_{H}=i d_{H} * S=\eta \circ \epsilon: H \rightarrow H .
$$

The tuple $(H, \mu, \eta, \Delta, \epsilon, S)$ is then called a Hopf algebra.

5. If $I$ is a semigoup (usually written as an addition), a Hopf algebra $H$ is called I-graded if $H=\bigoplus_{i \in I} H_{i}(i$ is called the degree) and both multiplication and comultiplication are additive with respect to the degree:

$$
\begin{aligned}
\mu\left(H_{i} \times H_{j}\right) & \subset H_{i+j}, \\
\Delta\left(H_{k}\right) & \subset \bigoplus_{i+j=k} H_{i+j} .
\end{aligned}
$$

6. If $M$ is a monoid, a $M$-graded Hopf algebra is called connected if its grade 0 component is the ground field $\mathbb{K}$.

For the shuffle Hopf algebra, these general objects can be defined as follows:

Definition 1.1.9 (deconcatenation coproduct, counit, antipode).

1. The deconcatenation coproduct is defined as the map

$$
\begin{aligned}
\Delta: \mathbb{K}\langle X\rangle & \rightarrow \mathbb{K}\langle X\rangle \otimes \mathbb{K}\langle X\rangle, \\
x_{1} \ldots x_{n} & \mapsto \sum_{k=0}^{n} x_{1} \ldots x_{k} \otimes x_{k+1} \ldots x_{n} .
\end{aligned}
$$

2. The counit is defined as the map $\epsilon: \mathbb{K}\langle X\rangle \rightarrow \mathbb{K}$ obtained by linear extension of the map $\epsilon: X^{*} \rightarrow \mathbb{K}$,

$$
\epsilon(x):=\left\{\begin{array}{ll}
1_{\mathbb{K}} & \text { if } x=1_{\emptyset} \\
0 & \text { if } x \in X^{*} \backslash X^{0}
\end{array} .\right.
$$

3. Finally, the antipode is defined as the map

$$
\begin{aligned}
S: \mathbb{K}\langle X\rangle & \rightarrow \mathbb{K}\langle X\rangle, \\
x_{1} \ldots x_{n} & \mapsto(-1)^{n} x_{n} \ldots x_{1} .
\end{aligned}
$$

The names given to the objects we just defined are justified by the following theorem. In fact, the shuffle Hopf algebra is an example of a Hopf algebra commonly encountered in books about Hopf algebras, for instance [HGK10, Example 3.4.6].

Theorem 1.1.10 (shuffle algebra is a Hopf algebra). ( $K\langle X\rangle, \mu, \eta, \Delta, \epsilon, S)$ is a commutative, but not cocommutative Hopf algebra. It is $\mathbb{N}_{0}$-graded by word length and connected.

Proof. A proof of this result can be found in [Lod94] or [HGK10]. 


\subsection{The Eulerian idempotent}

Definition 1.2.1 (Eulerian idempotent). Let $\left(H=\bigoplus_{n=1}^{\infty} H_{n}, \mu, \Delta, \eta, \epsilon\right)$ be a commutative graded connected Hopf algebra with convolution product $*$ over a field of characteristic 0 . Let further $f: H \rightarrow H$ be a $\mathbb{K}$-linear map that satisfies $f(1)=0$.

1. The first Eulerian idempotent eul ${ }^{(1)}$ is defined by

$$
\operatorname{eul}^{(1)}(f):=\ln _{*}(f+\eta \epsilon)=\sum_{i=1}^{\infty}(-1)^{i+1} \frac{f^{* i}}{i} .
$$

(where $\ln _{*}$ means that the power series of the natural logarithm is applied to the convolution product). Note that the above power series is actually finite because if $h \in H$ is an element of degree $n$, then $f^{* k}(h)=0$ for all $k>n$.

2. Higher Eulerian idempotents eul ${ }^{(k)}$ are defined by

$$
\operatorname{eul}^{(k)}(f):=\frac{\left(\mathrm{eul}^{(1)}(f)\right)^{* k}}{k !}
$$

By substituting $f:=i d-\eta \epsilon$, equation (1.22) immediately implies

$$
\mathrm{eul}^{(1)}(\mathrm{id}-\eta \epsilon)=\sum_{k \geq 1} \frac{(-1)^{k+1}}{k}(\mathrm{id}-\eta \epsilon)^{* k} .
$$

All subsequent mentions of the Eulerian idempotent will be with this particular $f$, and for convenience, we introduce some shorter notation:

$$
\begin{aligned}
e^{(k)} & :=\operatorname{eul}^{(k)}(\mathrm{id}-\eta \epsilon) \in \operatorname{End}_{\mathbb{K}}(H) \text { and } \\
e & :=e^{(1)} .
\end{aligned}
$$

The definition of the Eulerian idempotent implies some relations that hold for all Hopf algebras for which it has been defined above. Among them are the following ones:

Theorem 1.2.2 (properties of the Eulerian idempotent). Let $\left(H=\bigoplus_{n=1}^{\infty} H_{n}, \mu, \Delta, \eta, \epsilon\right)$ be a commutative graded connected Hopf algebra with convolution product $*$ over a field $\mathbb{K}$ of characteristic 0 . Also write $\cdot$ for the product $\mu$. Then the following assertions hold:

1. Denote by $e_{n}^{(i)}$ the $i$-th Eulerian idempotent restricted to homogeneous elements of degree $n$. Then:

$$
\left.\mathrm{id}^{* k}\right|_{H_{n}}=\left.\sum_{i=1}^{n} k^{i} e_{n}^{(i)}\right|_{H_{n}}
$$

for all $n \in \mathbb{N}_{+}$.

2.

$$
e_{n}^{(i)} e_{n}^{(j)}=\delta_{i j} e_{n}^{(i)}
$$

for all $n \in \mathbb{N}$, justifying the term "idempotent". 
3. Let $l, k \in \mathbb{N}_{+}$and $x_{1}, \ldots, x_{l} \in H \backslash H_{0}$. Then

$$
e^{(k)}\left(x_{1} \cdot \ldots \cdot x_{l}\right):=\left\{\begin{array}{ll}
0 & \text { if } k<l \\
\sum_{k_{1} \ldots k_{l} \in \mathbb{N}_{+} \text {with } \sum k_{i}=k} e^{\left(k_{1}\right)}\left(x_{1}\right) \cdot \ldots \cdot e^{\left(k_{l}\right)}\left(x_{l}\right) & \text { if } k \geq l
\end{array} .\right.
$$

4. All relations are homogeneous in the following sense: the finite sum

$$
\sum_{k=0}^{k_{\max }} \sum_{x_{1}, \ldots, x_{k} \in H} c_{x_{1}, \ldots, x_{k}} e\left(x_{1}\right) \cdot \ldots \cdot e\left(x_{k}\right)
$$

with coefficients $c_{x_{1}, \ldots, x_{k}} \in \mathbb{K}$ vanishes if and only if all the inner sums vanish themselves.

5. In addition to the original gradation, $H$ is also graded by homogeneity degree,

$$
\begin{aligned}
H & =\bigoplus_{k \in \mathbb{N}_{0}} H^{(k)}, \\
H^{(k)} & =\operatorname{span}_{\mathbb{K}}\left\{e\left(x_{1}\right) \cdot \ldots \cdot e\left(x_{k}\right) \mid x_{i} \in H\right\} .
\end{aligned}
$$

Proof. 1. follows from the definition using a relation between the power series of exp and $\log$ (See [Mei09, p. 37] for details.).

2. See [Lod94, Proposition 4.5.3].

3. Follows from 2 (see [BM11, p. 4]).

4. Follows from 3 (see [BM11, p. 4]).

5. Direct consequence of 4 .

We now leave the general theory of the Eulerian idempotent on Hopf algebra and consider the particular case of the shuffle Hopf algebra and some consequences of the above proposition. In the sequel, we will call the image of a word under the Eulerian idempotent an Euler element. Evaluating the proposition in our particular case, we conclude that we can re-write Euler elements in terms of words and vice versa.

Corollary 1.2.3. Let $x \in X^{n} \subset X^{*}$. Then (note that the $x_{i}$ used below are defined as words, not necessarily single letters):

1.

$$
x=\sum_{k=1}^{n} e^{(k)}(x)=\sum_{k=1}^{n} \frac{1}{k !} \sum_{\substack{x_{1}, \ldots, x_{k} \in X^{*} \backslash X^{0} \text { s.t. } \\ x_{1} \sim \ldots \frown x_{k}=x}} e\left(x_{1}\right) \# \ldots \# e\left(x_{k}\right)
$$

2. and

$$
e(x)=\sum_{k=1}^{n} \frac{(-1)^{k+1}}{k} \sum_{\substack{x_{1}, \ldots, x_{k} \in X^{*} \backslash X^{0} \\ x_{1} \simeq \ldots . x_{k}=x}} x_{1} \# \ldots \# x_{k} .
$$


Note that the factors of the shuffle products on the right hand side of the above equation (1.34) are words whose lengths add up to $n$. Therefore, $e\left(X^{n}\right) \subset \mathbb{K}\langle X\rangle_{n}$.

Since one special case of equation (1.29) is instrumental in the construction of a basis of ime, it is worth stating explicitly in the context of the shuffle Hopf algebra.

Corollary 1.2.4. Let $x, y \in X^{*} \backslash X^{0}$. Then

$$
e(x \# y)=0 .
$$

Proof. This is the special case of equation (1.29) for $l=2$. In this form, the equation was independently proved in [PR86, Proposition $5 \mathrm{f}$.].

There are also rules for reordering letters within Euler elements. The following theorem allows us to draw any letter to the beginning or end of a word. Unlike the other identities in this section, it does not hold in all Hopf algebras but seems to be specific to the shuffle Hopf algebra. This is due to the fact that in addition to the generic Hopf algebra product, a second product - the concatenation - is used. This situation is unusual in the conventional study of Hopf algebras but has in recent times been formalized using the notion of quadri-algebras introduced by M. Aguilar and J.-L. Loday [AL04].

Proposition 1.2.5 ("Proposition 7"). Let $x=x_{1} \ldots x_{n}$ in $X^{*}$. Then:

$$
e\left(x_{1} \ldots x_{n}\right)=(-1)^{i+1} e\left(x_{i}\left[\begin{array}{c}
x_{i-1}^{x_{i+1} \ldots x_{1}} \\
x_{n}
\end{array}\right)=(-1)^{n+j} e\left(\left[\begin{array}{l}
x_{1} \ldots x_{j-1} \\
x_{n} \ldots x_{j+1}
\end{array} x_{j}\right)=(-1)^{n+1} e\left(x_{n} \ldots x_{1}\right) .\right.\right.
$$

Proof. Originally proved in [PR86][Proposition 7.]. The assertion can be deduced from the recursive formula for the shuffle product 1.1.4 and corollary 1.2.4.

\subsection{Lyndon words and Euler-Lyndon elements}

Corollary 1.2.4 allowed Pohlmeyer and Rehren to formulate an algorithm to write any Euler element $e(x), x=x_{1} \ldots x_{n} \in X^{*}$ as a linear combination of Euler-Lyndon elements, and as we will see, the Euler-Lyndon elements are also linearly independent, hence a basis of the set of Euler elements. To appreciate this, we need to recapitulate some basic definitions.

The following two total orders on $X^{*}$ are extremely well known, but since they will show up in several places in this thesis, they deserve to be properly defined for the sake of completeness.

Definition 1.3.1 (lexicographic and graded lexicographic orders on words). Let $X$ be an alphabet with a total order $<_{X}$. Let $x=x_{1} \ldots x_{n}, y=y_{1}, \ldots, y_{m} \in X^{*}$ be words.

1. The lexicographic order (also universally familiar as the dictionary order) $<_{\text {Lex }}$ on $X^{*}$ is defined by

$$
x<_{\text {Lex }} y: \Leftrightarrow\left\{\begin{array}{l}
\exists k \in\{1, \ldots, \min (n, m)\} \text { such that } x_{i}=y_{i} \forall i \in\{1, \ldots, k-1\} \text { and } x_{k}<x \quad y_{k} . \\
x_{i}=y_{i} \forall i \in 1, \ldots n \text { and } n<m
\end{array} .\right.
$$


2. The graded lexicographic order $<$ DegLex on $X^{*}$ is defined by

$$
x<_{\text {DegLex }} y: \Leftrightarrow\left\{\begin{array}{l}
n<m \\
n=m \text { and } x<_{\text {Lex }} y
\end{array} .\right.
$$

Definition 1.3.2 (cyclic rotation, Lyndon word, Euler-Lyndon element).

1. Let $x=x_{1}, \ldots, x_{n} \in X^{n}$ be a word. Its cyclic rotations, also called shifts (to the left by $i$ positions), are the words

$$
x_{i+1} \ldots x_{n} x_{1} \ldots x_{i}
$$

where $i \in\{0, \ldots, n-1\}$.

2. Let $X$ be a totally ordered alphabet. A word that is uniquely minimal among its cyclic rotations is called a Lyndon word. The set of all Lyndon words over the alphabet $X$ is designated by $\operatorname{Lyn}(X)$, the set of all Lyndon words of length $n$ by $\operatorname{Lyn}(X, n)$.

3. We call the image of a Lyndon word under the Eulerian idempotent an Euler-Lyndon element.

In a slight misuse of notation, we sometimes call the Lyndon words occurring in EulerLyndon elements Euler-Lyndon words.

Lyndon words were discovered by A. SHIRshov (in 1953) and extensively studied by R. C. Lyndon (in 1954) [Lyn54], which is why the term "Lyndon-Shirshov words" is frequently used in the literature. They have a large number of applications in different areas of mathematics and computer science, and their theory is well developed. For instance, all words have a unique factorization (with respect to concatenation) into a nonincreasing sequence of Lyndon words (this is known as the Chen-Fox-Lyndon theorem), and there is an efficient algorithm [Reu93, section 7.3] that finds the successor (in the set of all Lyndon words up to a given length and with respect to the lexicographic order) of a given Lyndon word, which can be used to enumerate the set of all Lyndon words up to a given length.

The requirement of unique minimality among the cyclic rotations implies that periodic words cannot be Lyndon words. In the nomenclature used by POHLMEYer, Lyndon words are called cyclically minimal, a colloquialism for "uniquely cyclically minimal", which is always meant.

Now that the required definitions are in place, we can turn to PoHLMEYeR's and ReHren's algorithm, described in detail in [Poh89].

Remark 1.3.3 (Euler elements in terms of Euler-Lyndon elements). Let $e(x)$ be an Euler element and $x=x_{1} \ldots x_{n} \in X^{n}$ the corresponding word. The following algorithm rewrites $e(x)$ as a linear combination of Euler-Lyndon elements.

If $x$ already is a Lyndon word, we are finished. Otherwise, we begin by rearranging the letters of $x$ into words $y_{1}, \ldots, y_{r} \in X^{*}$ the following way.

How these $y_{i}$ are chosen depends on whether $x$ is periodic, i.e. if there exists a proper divisor $p$ of $n$ such that $x_{i}=x_{j}$ for all $i, j \in\{1, \ldots, n\}$ with $i=j \bmod p$ (the period of $x$ is the minimal such $p$ ). 
1. In the case that $x$ is nonperiodic, we can find a unique critical integer $i \in\{0, \ldots, n-1\}$ such that the word $x_{i+1} \ldots x_{n} x_{1} \ldots x_{i}$ is minimal among the cyclic rotations of $x$-i.e. a Lyndon word.

If $i=0$, then $x$ itself is already a Lyndon word.

Otherwise, set $y_{1}:=x_{1} \ldots x_{i}$ as well as $y_{2}:=x_{i+1} \ldots x_{n}$ and $c:=1$.

2. In the case that $x$ is periodic, we can similarly find a unique critical integer $i \in\{0, \ldots, p-1\}$ such that the word $x_{i+1} \ldots x_{p} x_{1} \ldots x_{i}$ is Lyndon.

Now, if $i>0$, again set $y_{1}:=x_{1} \ldots x_{i}$ as well as $y_{2}:=x_{i+1} \ldots x_{n}$ and $c:=1$.

Otherwise, set $c:=(n / p)$ ! and $y_{1}:=\ldots:=y_{n / p}=x_{1} \ldots x_{p}$.

Based on ideas by Rehren, Pohlmeyer showed ([יPoh89]) that the words $y_{1}, \ldots, y_{r}$ and the number $c$ defined above now have the property that

$$
\text { lead } e\left(y_{1} \# \ldots \# y_{r}\right)=c \cdot e(x)
$$

(leading with respect to the lexicographic order).

At the same time, by theorem 1.2.4. $e\left(y_{1} \# \ldots \# y_{r}\right)=0$, so

$$
e(x)=e(x)-\frac{1}{c} e\left(y_{1} \# \ldots \# y_{r}\right)=\sum_{z \in X^{n}} c_{z} e(z)
$$

with coefficients $c_{z} \in \mathbb{Q}$. Now, $c_{z} \neq 0$ is only possible for words $z$ that are permutations of $x$ and that also satisfy $z<x$ (w.r.t. lexicographic order) because of equation (1.40).

We then iterate this process for all Euler elements with nonzero coefficient occurring on the right hand side until all occuring words are Lyndon words.

Since there are only finitely many permutations of $x$ and the occurring words are decreased monotonously, the algorithm terminates. An implementation of this algorithm in Mathematica is given in B.2.)

Theorem 1.3.4 (Euler-Lyndon basis). Let $X$ be a totally ordered alphabet. Then the set $e(\operatorname{Lyn}(X))$ is basis of $\operatorname{im}(e) \subset \operatorname{Sh}(X)$.

Proof. This was proved by D. E. RADFORD in 1979 [Rad79] and formulated in terms of Lyndon words by G. Melançon and C. Reutenauer [Mel89].

Pohlmeyer's aproach yields another proof for finite alphabets, $X=\{0, \ldots, d-1\}$. By remark 1.3.3, the Euler-Lyndon elements of length $n$ span $(\operatorname{im} e)_{n}$. Pohlmeyer and Rehren proved [PR86, lemma, p. 602f] around the same time as Reutenauer that (presented here using the language established by BAHNs and MeINECKE)

$$
\operatorname{dim}(\operatorname{im} e)_{n}=\operatorname{NumLyndon}(n, d),
$$

which by theorem 1.3.6 is the number of Euler-Lyndon elements. Hence they form a basis. Of course this alternative proof is not particularly elegant, but it shows how the different approaches in the field can interrelate.

Since we have now established the Euler-Lyndon elements as a basis of im $e$, the dimension of a stratum of ime is given by the number of Lyndon words of a given length. Fortunately, the formula for this number is known and widely disseminated in the literature. 
Definition 1.3.5 (Möbius function). The Möbius function is defined as

$$
\begin{aligned}
\mu: \mathbb{N}_{+} & \rightarrow\{-1,0,1\}, \\
n & \mapsto \begin{cases}+1 & \text { if } n \text { is square free and the number of prime factors of } n \text { is even } \\
-1 & \text { if } n \text { is square free and the number of prime factors of } n \text { is odd } . \\
0 & \text { if } n \text { is not square free }\end{cases}
\end{aligned}
$$

Theorem 1.3.6 (number of Lyndon words).

1. The number of Lyndon words of length $n$ from an finite alphabet of $d$ letters is

$$
\operatorname{NumLyndon}(n, d):=\frac{1}{n} \sum_{q \mid n} \mu(q) d^{n / q} .
$$

2. The number of Lyndon words where the letters $a_{i}$ occur exactly $n_{i}, i \in\{1, \ldots, p\}$ times is

$$
\frac{1}{n} \sum_{q \mid n_{i} \forall i} \mu(q)\left(\begin{array}{c}
n / q \\
n_{1} / q, \ldots, n_{p} / q
\end{array}\right),
$$

where $n:=\sum_{i} n_{i}$, using the multinomial coefficient defined as

$$
\left(\begin{array}{c}
n \\
k_{1}, \ldots, k_{p}
\end{array}\right):=\frac{n !}{k_{1} ! \cdot \ldots \cdot k_{p} !} .
$$

Proof. [Reu93][Theorem 7.1] gives these formulas for the number of primitive necklaces with the same restriction on letters as given in the premise of this theorem.

Necklaces are words modulo the cyclic rotation, so the map that maps a necklace to the

\begin{tabular}{|c|c|c|c|c|c|c|c|c|c|c|c|}
\hline \multirow{2}{*}{\multicolumn{2}{|c|}{$\operatorname{NumLyndon}(n, d)$}} & \multicolumn{10}{|c|}{$n$} \\
\hline & & 1 & 2 & 3 & 4 & 5 & 6 & 7 & 8 & 9 & 10 \\
\hline \multirow{10}{*}{ d } & 1 & 1 & 0 & 0 & 0 & 0 & 0 & 0 & 0 & 0 & 0 \\
\hline & 2 & 2 & 1 & 2 & 3 & 6 & 9 & 18 & 30 & 56 & 99 \\
\hline & 3 & 3 & 3 & 8 & 18 & 48 & 116 & 312 & 810 & 2184 & 5880 \\
\hline & 4 & 4 & 6 & 20 & 60 & 204 & 670 & 2340 & 8160 & 29120 & 104754 \\
\hline & 5 & 5 & 10 & 40 & 150 & 624 & 2580 & 11160 & 48750 & 217000 & 976248 \\
\hline & 6 & 6 & 15 & 70 & 315 & 1554 & 7735 & 39990 & 209790 & 1119720 & 6045837 \\
\hline & 7 & 7 & 21 & 112 & 588 & 3360 & 19544 & 117648 & 720300 & 4483696 & 28245840 \\
\hline & 8 & 8 & 28 & 168 & 1008 & 6552 & 43596 & 299592 & 2096640 & 14913024 & 107370900 \\
\hline & 9 & 9 & 36 & 240 & 1620 & 11808 & 88440 & 683280 & 5380020 & 43046640 & 348672528 \\
\hline & 10 & 10 & 45 & 330 & 2475 & 19998 & 166485 & 1428570 & 12498750 & 111111000 & 999989991 \\
\hline
\end{tabular}
word that is minimal among its cyclic rotations is a bijection. Primitivity of necklaces is the same as nonperiodicity of words, so this bijection actually maps the set of primitive necklaces bijectively to the set of Lyndon words.

For easy reference, a table of $\operatorname{NumLyndon}(n, d)$ for low $n$ and $d$ is provided.

Table 1.1: NumLyndon $(n, d)$ for low $n$ and $d$. 


\subsection{The Pohlmeyer-Rehren Lie algebra}

Before we begin constructing the Pohlmeyer-Rehren Lie algebra, let us recall some very basic definitions and facts for easy reference.

Definition 1.4.1. (Lie algebra, direct sum, simple, semisimple, Poisson algebra, derived series, solvable, lower central series, nilpotent, Poisson algebra, generation)

1. Let $G$ be vector space over a field $\mathbb{K}$. A $\mathbb{K}$-bilinear map $[\cdot, \cdot]: G \times G \rightarrow G$ is called a Lie bracket if

$$
\begin{aligned}
{[x, x] } & =0 \text { (alternativity) and } \\
{[x,[y, z]]+[y,[z, x]]+[z,[x, y]] } & =0 \text { (Jacobi identity) }
\end{aligned}
$$

are satisfied for all $x, y, z \in G$. Then $(G,[\cdot, \cdot])$ (or more concisely only $G$, if the Lie bracket is unambiguous) is called a Lie algebra (over $\mathbb{K}$ ). In particular, any Lie algebra is an algebra and all terms defined for algebras such as the ones from definition 1.1.6 apply. Because of equation (1.46), $[x, y]=-[y, x]$ for all $x, y \in G$ and abelian Lie algebras are those with the trivial Lie bracket 0.

2. The direct sum of Lie algebras $\left(G,[\cdot, \cdot]_{G}\right)$ and $\left(H,[\cdot, \cdot]_{H}\right)$ is defined as the vector space $G \oplus H$ with the Lie bracket $[\cdot, \cdot]: G \oplus H \times G \oplus H \rightarrow G \oplus H$ defined by

$$
\left[(g, h),\left(g^{\prime}, h^{\prime}\right)\right]:=\left(\left[g, g^{\prime}\right]_{G},\left[h, h^{\prime}\right]_{H}\right) \quad \forall g, g^{\prime} \in G, h, h^{\prime} \in H .
$$

3. A Lie algebra $G$ that has no ideals except 0 and $G$ itself is called simple. A Lie algebra that is a direct sum of simple Lie algebras is called semisimple.

4. Let $(G,[\cdot, \cdot])$ be a Lie algebra. Iteratively define two series of ideals, the derived series

$$
G^{(0)}:=G, \quad G^{(n+1)}:=\left[G^{(n)}, G^{(n)}\right] \forall n \in \mathbb{N}_{0}
$$

of $G$ and the lower central series

$$
G^{0}:=G, \quad G^{n+1}:=\left[G, G^{n}\right] \forall n \in \mathbb{N}_{0}
$$

of $G$. If $G^{(n)}=0$ for some $n \in \mathbb{N}_{0}$, then $G$ is called solvable. If $G^{n}=0$ for some $n \in \mathbb{N}_{0}$, then $G$ is called nilpotent. From the definition, one concludes that nilpotent Lie algebras are solvable.

5. Let $(G,\{\cdot, \cdot\})$ be a Lie algebra and let $(G, \cdot)$ be an associative algebra. If

$$
\{x \cdot y, z\}=x \cdot\{y, z\}+\{x, z\} \cdot y \text { for all } x, y, z \in G,
$$

then $(G,\{\cdot, \cdot\}, \cdot)$ is called a Poisson algebra and the Lie bracket $\{\cdot, \cdot\}$ is called a Poisson bracket.

6. Let $G$ be a (Lie) algebra / Poisson algebra / group / ideal with a subset $X \subset G$. Then $G$ is called generated (as a (Lie) algebra / Poisson algebra / group / ideal) by $X$ if no subset $H \varsubsetneqq G$ exists such that $X \subset H$ and $H$ is a (Lie) algebra / Poisson algebra / group / ideal. If a finite set $X$ exists such that $X$ generates $G$, then $G$ is called finitely generated. 
The Pohlmeyer-Rehren Lie algebra and its structure constitute a major focus of this thesis. It can be constructed from the shuffle algebra $S h(X)$ by equipping it with some additional structure. We begin by defining two closely related ${ }^{3}$ derivations of $\operatorname{Sh}(X)$ and then use them to define a Lie bracket on $\operatorname{im}(e)$.

\section{Theorem 1.4.2.}

1. Let $a \in X$. The maps $\partial_{a}^{R}, \partial_{a}^{L}: \operatorname{Sh}(X) \rightarrow \operatorname{Sh}(X)$ defined (on $X^{*}$, and on $\operatorname{Sh}(X)$ by linear extension)

$$
\begin{aligned}
\partial_{a}^{R}\left(x_{1} \ldots x_{n}\right) & :=\delta_{a, x_{1}} x_{2} \ldots x_{n}, \\
\partial_{a}^{L}\left(x_{1} \ldots x_{n}\right) & :=\delta_{a, x_{n}} x_{1} \ldots x_{n-1}, \\
\partial_{a}^{R}\left(1_{\emptyset}\right) & :=\partial_{b}^{L}\left(1_{\emptyset}\right):=0
\end{aligned}
$$

are derivations of $\operatorname{Sh}(X)$.

2. Let $X=\{0, \ldots, d-1\}$ and let $g$ be a symmetric $d \times d$-matrix (where rows and columns are indexed by X). Then the map $[\cdot, \cdot]: \operatorname{im}(e) \times \operatorname{im}(e) \rightarrow \operatorname{im}(e)$,

$$
[e(x), e(y)]:= \begin{cases}\sum_{a, b \in X} g_{a, b} e\left(\partial_{a}^{R} * S(x) \frown S * \partial_{b}^{L}(y)\right) & \text { if } n, m>1 \\ 0 & \text { if } n=1 \text { or } m=1\end{cases}
$$

is a Lie bracket.

3. In terms of Euler elements, this Lie bracket can be written as

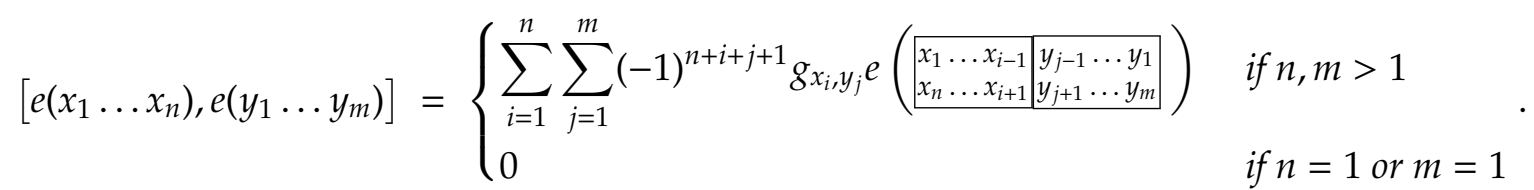

4. The Lie algebra $\mathfrak{g}:=(\operatorname{im} e,[\cdot, \cdot])$ is graded by word length-2;

$$
\begin{gathered}
\operatorname{deg} e\left(x_{1} \ldots x_{n}\right):=n-2 ; \\
\mathfrak{g}=\bigoplus_{l \geq-1} \mathfrak{g}_{l,} \\
{\left[\mathfrak{g}_{l,}, \mathfrak{g}_{l^{\prime}}\right] \subset \mathfrak{g}_{l+l^{\prime}} \text { for all } l, l^{\prime} \in \mathbb{N}_{0} \cup\{-1\}}
\end{gathered}
$$

where $\mathfrak{g}_{l}:=\{g \in \mathfrak{g} \mid \operatorname{deg} g=l\}$.

Proof. (mainly following [BM11]):

1. This follows directly from lemma 1.1.4.

\footnotetext{
${ }^{3} \operatorname{In}$ fact, $\partial_{a}^{R}=-S \circ \partial_{a}^{L} \circ S$
} 
2. This is can be demonstrated using 3: Alternativity then is a consequence of proposition 1.2.5 and the Jacobi identity can be proved by tracking the contributions of the cyclic product in the Jacobi identity according to the $g_{a, b}$ occurring, observing that they cancel for certain terms, and then using 1.2 .5 to rewrite all other contributions into such terms (see [BM11, Prop. 3] for details).

3. We evaluate the convolution product (notice the index shift for $j$ ):

$$
\begin{aligned}
& {[e(x), e(y)]=\sum_{a, b \in X} g_{a, b} e\left(\partial_{a}^{R} * S(x) \frown S * \partial_{b}^{L}(y)\right)} \\
& \stackrel{1.15}{=} \sum_{a, b \in X} g_{a, b} e\left(\mu \circ\left(\partial_{a}^{R} \otimes S\right) \circ \Delta(x) \frown \mu \circ\left(S \otimes \partial_{b}^{L}\right) \circ \Delta(y)\right) \\
& \stackrel{1.19}{=} \sum_{a, b \in X} g_{a, b} \sum_{i=0}^{n} \sum_{j=1}^{m+1} e\left(\begin{array}{l|l}
\mid \begin{array}{ll}
\partial_{a}^{R}\left(x_{1} \ldots x_{i}\right) \\
S\left(y_{i+1} \ldots x_{n}\right)
\end{array} & \left.\begin{array}{l}
\left(y_{b} \ldots y_{j-1}\left(y_{j} \ldots y_{m}\right)\right. \\
S\left(x_{i}\right)
\end{array}\right) \\
\hline
\end{array}\right. \\
& =\sum_{a, b \in X} g_{a, b} \sum_{i=1}^{n} \sum_{j=1}^{m} \delta_{a, x_{i}} \delta_{b, y_{j}}(-1)^{n-i}(-1)^{j-1} e\left(\begin{array}{l|l|l}
x_{1} \ldots x_{i-1} & \begin{array}{l}
y_{j-1} \ldots y_{1} \\
x_{n} \ldots x_{i+1} \\
y_{j+1} \ldots y_{m}
\end{array}
\end{array}\right) \\
& =\sum_{i=1}^{n} \sum_{j=1}^{m}(-1)^{n+i+j+1} g_{x_{i}, y_{j}} e\left(\begin{array}{l|l|l|}
x_{1} \ldots x_{i-1} & y_{j-1} \ldots y_{1} \\
x_{n} \ldots x_{i+1} & y_{j+1} \ldots y_{m}
\end{array}\right) \text {. }
\end{aligned}
$$

4. This is a simple counting argument: The word on the right hand side of equation (1.54) has two fewer letters than the words on the words on the left hand side have combined.

Note that the Lie bracket can also be understood as a sum over all possibilities which letters of $x$ to move to the right and of $y$ to move to the left using PoHLMEYer and ReHren's proposition 7 1.2.5), then deleting the moved letters with the derivations $\partial_{a}^{R}$ and $\partial_{a}^{L}$, weighing the summand with the entry of $g$ corresponding to the deleted letters and concatenating the results.

Definition 1.4.3 (Pohlmeyer-Rehren Lie algebra). If $g$ is proportional to the metric tensor $\eta$ of the Minkowski metric, i.e.

$$
g=\alpha \eta=\alpha \operatorname{Diag}(-1, \underbrace{1, \ldots, 1}_{d-1})
$$

with $\alpha \in \mathbb{C} \backslash\{0\}$ (where Diag denotes a diagonal matrix, its arguments being the entries of the diagonal), we call $\mathfrak{g}:=(\operatorname{im}(e),[\cdot, \cdot])$ the Pohlmeyer-Rehren Lie algebra.

For reasons that will be discussed later in remark 3.1 .2 the cases $d=3$ and $d=4$ will be of particular interest. We will later see (in section 1.8) how a decomposition of $g$ can be used to bring to bear some combinatorial arguments on the words involved.

Remark 1.4.4. Note that the lowest nonzero stratum of $\mathfrak{g}$ is not $l=0$ but $l=-1$. Since $\mathfrak{g}_{-1}$ is central by the defining equation of the Lie bracket (1.54), it is often not considered explicitly. In this sense, $\mathfrak{g}$ can be thought of as a $\mathbb{N}_{0}$-graded Lie algebra with added central elements instead of a $\mathbb{Z}$-graded Lie algebra in which all strata of degree less than -1 are zero. 
Remark 1.4.5. Since the Pohlmeyer-Rehren Lie algebra is the primary focus of this thesis, its history warrants some attention. It was first described by PoHLMEYER and REHREN ([PR86]). There, the Lie bracket was called the "modified Poisson bracket", and Lie algebra was not explicitly named, but its elements were called "truncated tensors". The context in which these notions were developed was PoHLMEYeR's approach to the quantization of the NAmbuGото string. The truncated tensors were defined by way of path-ordered integrals of the so called left and right movers, which are tangent vectors on the string's world surface. Many of the facts used in this thesis were proved in that context.

The considerably simpler definition used here is taken from BAHNS and Meinecke, who proved it to be equivalent to PohlmeYer's and Rehren's definition ([BM11, ]). Since it was not the focus of their work, they simply call it the "auxiliary Lie algebra", but since its structure is the main subject of this thesis and the term "auxiliary Lie algebra" will be used for an unrelated object in the context of Kac-Moody algebras later, it deserves its proper name, at least within this scope.

A more detailed account of the backdrop of the quantization of the Nambu-Goto string in which $g$ was discovered and the subsequent constructions in which it is featured is given in chapter 3 .

Remark 1.4.6. Using any other diagonal matrix with complex nonzero entries instead of $\alpha \eta$ in definition 1.4.3 of $\mathfrak{g}$ leads to an isomorphic Lie algebra; in this case the basis vectors can be multiplied with appropriate complex numbers to obtain the same structure constants. In Pohlmeyer's original work recounted in chapter 3 , the Minkowski metric is inherited from the physical problem of string quantization where it is the metric of $d$-dimensional spacetime (with one time dimension). We continue this special treatment of the time dimension by using the Minkowski metric in the definition of $\mathfrak{g}$ as well as some other special considerations of the letter 0 in our alphabet later.

In Pohlmeyer's original work $\alpha=2$ is used due to the way the Lie bracket is constructed there, but since multiplying the structure constants of a Lie algebra with a global nonzero factor is an isomorphism of Lie algebras, this is not relevant to the structure of $\mathfrak{g}$.

\subsection{Measures of growth of the Pohlmeyer-Rehren Lie algebra}

Corollary 1.5.1. 1. The dimension of $\mathfrak{g}_{l}$ (the l-th stratum of $\mathfrak{g}$ ) is

$$
\operatorname{dim} g_{l}=\frac{1}{l+2} \sum_{q \mid l+2} \mu(q) d^{(l+2) / q} .
$$

2. The Hilbert series of the Lie algebra $\mathfrak{g}_{\geq 1}=\bigoplus_{l+1}^{\infty} \mathfrak{g}_{l}$ is

$$
\sum_{l=1}^{\infty} \operatorname{dim} \mathfrak{g}_{l} t^{l}=\sum_{l=1}^{\infty} \frac{1}{l+2} t^{l} \sum_{q \mid l+2} \mu(q) d^{(l+2) / q} .
$$

3. The Hilbert series of the universal enveloping algebra of $\mathfrak{g}_{l \geq 1}$ is

$$
\prod_{l=1}^{\infty} \frac{1}{\left(1-t^{l}\right)^{\operatorname{dim} g_{l}}}=\prod_{l=1}^{\infty} \frac{1}{\left(1-t^{l}\right)^{\frac{1}{l+2} \sum_{q \mid l+2} \mu(q) d^{(l+2) / q}} .}
$$


Proof. 1. This follows immediately from theorem 1.3.61 and the definition of the gradation on $\mathfrak{g}$.

2. The left hand side of the equation is the Hilbert series of a graded algebra. Then use 1.

3. Similarly, the left hand side gives the Hilbert series of the universal enveloping algebra of a Lie algebra (see [Ufn98, S. 260 f.]).

Remark 1.5.2. Although we will not use the Hilbert series of $\mathfrak{g}_{\geq 1}$ in the sequel, it is given explicitly since there are many connections between the Hilbert series and other properties of a graded algebra (see [Ufn98] and [Ani82]). For instance by a theorem due to Govorov, the Hilbert series of any monomial finitely presented algebra is a rational function Ani82, Theorem 1.3]. Note that we have the following estimate of the Hilbert series of $\mathfrak{g}$ :

$$
\begin{aligned}
H_{\mathfrak{g}} & =\sum_{l=1}^{\infty} \frac{1}{l+2} t^{l} \sum_{q \mid l+2} \mu(q) d^{(l+2) / q} \\
& <\sum_{l=1}^{\infty} \frac{1}{l+2} t^{l} d^{l+2} \\
& =\frac{1}{t^{2}} \sum_{l=1}^{\infty} \frac{1}{l+2}(t d)^{l+2} \\
& =\frac{1}{t^{2}}\left(-t d-\frac{1}{2}(t d)^{2}+\sum_{l=1}^{\infty} \frac{1}{l}(t d)^{l}\right) \\
& =\frac{1}{t^{2}}\left(-t d-\frac{1}{2}(t d)^{2}-\ln (1-t d)\right) .
\end{aligned}
$$
$d=2$.

We can use the growth of $\mathfrak{g}$ for an observation about the exotic (cf. remark 3.1.2) case of

Proposition 1.5.3. In the case of $d=2, \mathfrak{g}_{1}$ does not generate $\mathfrak{g}$ (as a Lie algebra).

Proof. Due to antisymmetry, $\operatorname{dim}\left[\mathfrak{g}_{1}, \mathfrak{g}_{1}\right]=\frac{2 \cdot 1}{2}=1$, but $\operatorname{dim} \mathfrak{g}_{2}=3$.

Note that for $d>2$, this argument fails, since

$$
\frac{\operatorname{dim} \mathfrak{g}_{1} \cdot\left(\operatorname{dim} \mathfrak{g}_{1}-1\right)}{2}=\frac{1}{18}\left(d^{3}-d\right)\left(d^{3}-d-3\right)>\frac{1}{4}\left(d^{4}-d^{2}\right)=\operatorname{dim} \mathfrak{g}_{2}
$$

In fact, for $d=3$, explicit calculation (cf. page 67) yields the fact that $\left[\mathfrak{g}_{1}, \mathfrak{g}_{1}\right] \supset \mathfrak{g}_{2}$ (the other inclusion is trivial since $\mathfrak{g}$ is graded).

Since the (polynomial) degree of the left side is higher for increasing $l$, this approach will not be fruitful for higher $l$ as well. 


\subsection{The spin basis}

Definition 1.6.1 (spin basis). Let $X=\{0, \ldots d-1\}$ and let $\mathfrak{g}$ be the corresponding PohlmeyerRehren Lie algebra. We define the elements of the word algebra $\mathbb{C}\langle X\rangle$

$$
\pm_{i}:=\frac{1}{\sqrt{2}}((2 i-1) \pm \mathbf{i}(2 i))
$$

(the terms in the inner parentheses are letters and $\mathbf{i}$ is the imaginary unit) for $i \in\{1 \ldots\lfloor(d-1) / 2\rfloor\}$ and, if $d$ is even, additionally

$$
\pm_{0}:=\frac{1}{\sqrt{2}}( \pm(0)+(d-1)) \text {. }
$$

Using linearity of concatenation, this can be extended first to $\mathbb{C}(X)$ and then, using equation (1.34) and linearity of the shuffle product, to im $e$. For the sake of convenience, we now define the index sets

$$
I_{d}:=\left\{\begin{array}{ll}
\{1, \ldots,(d-1) / 2\} & \text { if } d \text { is odd } \\
\{0, \ldots,(d-2) / 2\} & \text { if } d \text { is even }
\end{array} .\right.
$$

Further define the alphabet

$$
X_{ \pm}:=\left\{0, \pm_{i} \mid i \in I_{d}\right\}
$$

if $d$ is odd and

$$
X_{ \pm}:=\left\{ \pm_{i} \mid i \in I_{d}\right\}
$$

if $d$ is even.

Since the above linear transformations are invertible, we can replace all letters of $X$ by letters of $X_{ \pm}$and obtain the same construction of the Pohlmeyer-Rehren Lie algebra. The only difference is that the metric tensor $g$ changes into

$$
g_{x, y}= \begin{cases}-\alpha & \text { if } x= \pm_{i}, y=\mp_{i} \text { for some } i \\ \alpha & \text { if } x=y=0 \quad \text { (note that this only occurs for odd } d \text { ) } \\ 0 & \text { else. }\end{cases}
$$

under the above linear transformation. This, again, is a symmetric $d \times d$ matrix, so we can continue with the construction of the Pohlmeyer-Rehren algebra unimpeded and find, using the same arguments as in chapter 1, that a basis of $\mathfrak{g}$ is given by the Euler-Lyndon elements with respect to $X_{ \pm}$. This is known as the spin basis or \pm basis of $\mathfrak{g}$. Unless stated otherwise, the order

$$
0<-{ }_{1}<+{ }_{1}<--_{2}<\ldots
$$

(for odd $d$ ) or

$$
-{ }_{0}<+_{0}<-{ }_{1}<+_{1}<--_{2}<\ldots
$$

(for even $d$ ) is used on $X_{ \pm}$. 
Remark 1.6.2. We could simply have chosen appropriate letters and defined our symmetric bilinear form $g$ to act this way in the first place. Due to the way the Euler elements (in the language used there, "truncated tensors") have been defined traditionally (connected to the dimensions of Minkowski spacetime) and the suggestive nature of plusses and minuses which will become apparent later on, we keep the traditional notation for the spin basis that goes back at least until [Hap93].

Likewise, the special consideration the letter 0 received in the traditional notation is not neccessary in light of the isomorphism from remark 1.4.6, but kept and built upon by introducing $I_{d}$ to maintain consistency.

Using the spin basis has some advantages: since the Lie bracket eliminates corresponding pairs of plusses and minusses, the differences between the numbers of plusses and corresponding minusses is not changed by the Lie bracket. We capture this fact in a definition.

Definition 1.6.3 (magnetic quantum numbers). Let $x=x_{1} \ldots x_{n} \in X_{ \pm}^{*}$. Define its magnetic quantum numbers

$$
m_{i}(x):=\sum_{j=1}^{n} \delta_{+_{i} x_{j}}-\delta_{-i x_{j}}
$$

for $i \in I_{d}$. We designate the ordered tuple of all magnetic quantum numbers with the letter $m(x)$. Often, the argument is suppressed (and for $d=3$, the index as well). Since the magnetic quantum numbers are invariant under the shuffle product, concatenation and deconcatenation (if the magnetic quantum number w.r.t. some $i$ of such a product is understood as the sum of all the occurring magnetic quantum numbers w.r.t. the same $i$ ), they can be extended in a straightforward way to Euler elements: $m(x)=m(e(x))$.

Lemma 1.6.4 (magnetic quantum numbers as a gradation). Further define $\mathrm{g}^{m}$ and $\mathfrak{g}_{l}^{m}$ as the subsets of elements of $\mathfrak{g}$ and $\mathfrak{g}_{l}$ respectively with magnetic number tuple $m$. Then the quadratic quantum numbers can be understood as a gradation:

1.

$$
\begin{aligned}
& \mathfrak{g}_{l}=\bigoplus_{m \text { with } \sum\left|m_{i}\right| \leq l+2} \mathfrak{g}_{l}^{m}, \\
& {\left[\mathfrak{g}_{l}^{m}, \mathfrak{g}_{l^{\prime}}^{m^{\prime}}\right] \subset \mathfrak{g}_{l+l^{\prime}}^{m+m^{\prime}} . }
\end{aligned}
$$

2.

$$
\begin{aligned}
& \mathfrak{g}=\bigoplus_{m} \mathfrak{g}^{m}, \\
& {\left[\mathfrak{g}^{m}, \mathfrak{g}^{m^{\prime}}\right] \subset \mathfrak{g}^{m+m^{\prime}} . }
\end{aligned}
$$

3. If $l \geq 0$ and $x \in \mathrm{g}_{l}$, then for all $i$, we have $\left|m_{i}\right| \leq l+1$.

4. If $d$ is even and $x \in \mathfrak{g}_{l}$, then $\sum_{i} m_{i}=l \bmod 2$. 
Proof. The sum in the first equation is direct because the Euler-Lyndon elements are a basis of $\mathfrak{g}$, each element of which has a defined tuple of magnetic quantum numbers. The rest of 1 , as well as 2 and 4 follow from simple counting arguments related to definition 1.6.3 about the numbers of plusses and minusses in a word. For 3 , we additionally observe that a word consisting of only a repetition of a single letter can be written as a shuffle product, more precisely

$$
x^{n}=\frac{1}{n}\left[\begin{array}{c}
x \\
x^{n-1}
\end{array}\right]
$$

for all $x \in X$ and $n \in \mathbb{N}_{+}$, so by lemma 1.2.4, $e(x \ldots x)=0$.

The magnetic quantum numbers can be obtained not only by counting letters, but they also occur in eigenvalues in the diagonalization of the adjunctions of particular elements of $\mathfrak{g}_{0}$.

Lemma 1.6.5. The elements of the spin basis are eigenvectors of the adjunction of the elements

$$
\tilde{h}_{i}:=\frac{1}{\alpha} e\left(-{ }_{i}+{ }_{i}\right) \text { for } i \in I_{d} .
$$

The corresponding eigenvalue of the eigenvector $e\left(x_{1} \ldots x_{n}\right)\left(\right.$ of $\left.\operatorname{ad}_{\tilde{h}_{i}}\right)$ is $m_{i}$.

Proof.

$$
\begin{aligned}
& \operatorname{ad}_{\tilde{h}_{i}} e\left(x_{1} \ldots x_{n}\right)=\left[\frac{1}{\alpha} e\left(-{ }_{i}+{ }_{i}\right), e\left(x_{1} \ldots x_{n}\right)\right] \\
& \stackrel{1.54}{=} \frac{1}{\alpha} \sum_{j=1}^{n}(-1)^{2+1+j+1} g_{-_{i} x_{j}} e\left(+i \stackrel{\left.\begin{array}{l}
x_{x_{j-1} \ldots x_{1}} \\
x_{j+1} \ldots x_{n}
\end{array}\right)}{)}\right. \\
& +(-1)^{2+2+j+1} g_{+_{i} x_{j}} e\left(--_{i} \begin{array}{l}
x_{j-1} \ldots x_{1} \\
x_{j+1} \ldots x_{n}
\end{array}\right) \\
& =\sum_{j=1}^{n}\left(\delta_{+_{i} x_{j}}-\delta_{-_{i} x_{j}}\right)(-1)^{j+1} e\left(x_{j}\left[\begin{array}{l}
\begin{array}{l}
x_{j-1} \ldots x_{1} \\
x_{j+1} \ldots x_{n}
\end{array} \\
x^{\prime}
\end{array}\right)\right. \\
& \stackrel{1.2 .5}{=} \sum_{j=1}^{n}\left(\delta_{x_{j}+i}-\delta_{x_{j-i}}\right) e\left(x_{1} \ldots x_{N}\right) \\
& \stackrel{1.72}{=} m_{i} \cdot e\left(x_{1} \ldots x_{n}\right) \text {. }
\end{aligned}
$$

While we have now proved these facts with combinatorial methods, they will be a stepping stone towards the representation theory that will be outlined in chapter 2 . There, we will see that the $\tilde{h}_{i}$ span a Cartan subalgebra of $\mathfrak{g}_{0}$ and understand the magnetic quantum numbers in terms of the more standard notions of roots and weights that stem from representation theory. 


\subsection{The Pohlmeyer-Rehren Lie algebra is not Kac-Moody}

Kac-Moody algebras are a class of algebras that have some similarities to the Pohlmeyer-Rehren Lie algebra's proven or conjectured properties.

- They are frequently infinite-dimensional.

- They have root space decompositions, similar to the one described in subsection 2.2.3.

- They are generated by a subalgebra (their Cartan subalgebra) and a finite number of generators.

Kac-Moody algebras are also a widely researched topic, so a large body of results already exists that could serve as a toolkit facilitating the exploration and classification of the Pohlmeyer-Rehren Lie algebra. Therefore, the question if the Pohlmeyer-Rehren Lie algebra is Kac-Moody is of great interest in its study.

It could be proved (corollary 1.7.3) that the Pohlmeyer-Rehren Lie algebra is not a KacMoody algebra. To provide some background as well as notation for the proof, we recapitulate the definition of Kac-Moody algebras from KAc's book about them [Kac82][chapter 1], omitting a significant part of the theory that he intersperses but that will not be needed for the proof.

Definition 1.7.1 (Kac-Moody algebra). 1. A matrix $A=\left(a_{i j}\right) \in \operatorname{Mat}(n \times n, \mathbb{C})$ of $\operatorname{rank} l$ is called a generalized Cartan matrix if it satisfies

$$
\begin{aligned}
& a_{i i}=2 \text { for all } i \in\{1, \ldots, n\}, \\
&-a_{i j} \in \mathbb{N}_{0} \text { for } i \neq j, \\
& a_{i j}=0 \Leftrightarrow a_{j i}=0 .
\end{aligned}
$$

2. A realization of $A$ is a triple $\left(\mathfrak{h}, \Pi, \Pi^{\vee}\right)$ of a $\mathbb{C}$-vector space $\mathfrak{h}$ and indexed subsets $\Pi=\left\{\alpha_{1}, \ldots \alpha_{n}\right\} \subset \mathfrak{h}^{*}$ and $\Pi^{\vee}=\left\{\alpha_{1}^{\vee}, \ldots \alpha_{n}^{\vee}\right\} \subset \mathfrak{h}$ such that

$$
\begin{aligned}
& \Pi \text { and } \Pi^{\vee} \text { are linearly independent, } \\
& \begin{aligned}
\alpha_{j}\left(\alpha_{i}^{\vee}\right) & =a_{i j}, \\
n-l & =\operatorname{dim} \mathfrak{h}-n .
\end{aligned}
\end{aligned}
$$

$\Pi$ is called a root basis and $\Pi^{\vee}$ is called a coroot basis and their elements simple roots or simple coroots respectively.

3. The auxiliary Lie algebra $\tilde{\mathfrak{g}}(A)$ is defined as the Lie algebra freely generated by $\mathfrak{h}$ and the finite set of Chevalley generators $4\left\{x_{i}, y_{i} \mid i \in\{1, \ldots, n\}\right\}$ modulo the ideal generated by the relations

$$
\begin{aligned}
{\left[x_{i}, y_{j}\right] } & =\delta_{i j} \alpha_{i}^{\vee}, \\
{\left[h, h^{\prime}\right] } & =0, \\
{\left[h, x_{i}\right] } & =\alpha_{i}(h) x_{i}, \\
{\left[h, y_{i}\right] } & =-\alpha_{i}(h) y_{i}
\end{aligned}
$$

for all $i, j \in\{1, \ldots, n\}$ and $h, h^{\prime} \in \mathfrak{h}$.

\footnotetext{
${ }^{4}$ This means that their structure constants are integers.
} 
4. Let $\mathfrak{r}$ be the (unique) maximal ideal of $\tilde{\mathfrak{g}}$ intersecting $\mathfrak{h}$ trivially. We define the quotient Lie algebra

$$
\mathfrak{g}(A):=\tilde{\mathfrak{g}}(A) / \mathfrak{r},
$$

and call it a Kac-Moody algebra 5 . We identify $\mathfrak{h}, x_{i}, y_{i}$ with their images under the canonical projection.

In order to see that the Pohlmeyer-Rehren Lie algebra $g$ is not Kac-Moody, we now use this definition to prove and make use of a more general fact about Kac-Moody algebras that, although alluded ${ }^{6}$ to in Kac's book, is not spelled out there explicitly.

Theorem 1.7.2. A Lie algebra with a nontrivial gradation in which all elements of negative degree are central is not a Kac-Moody algebra.

Proof. Proof by contradiction. Assume said Lie algebra is Kac-Moody and call it $\mathfrak{g}(A)$.

1. Then $\mathfrak{g}(A)$ is generated by the Cartan subalgebra $\mathfrak{h}$ and Chevalley generators $x_{i}, y_{i}$. If all these elements had degree zero, there could not be elements of $\mathfrak{g}(A)$ with positive degree. So there is an element of $\mathfrak{h} \cup\left\{x_{i}, y_{i} \mid i=1 . . n\right\}$ with positive degree.

2. There is an element $h \in \mathfrak{h}$ with $\operatorname{deg}(h)>0$. Suppose this were false. Then, at least one of the Chevalley generators $x_{p}$ or $y_{p}$ has positive degree. Then

$$
\left[x_{p}, y_{p}\right]=\alpha_{p} \neq 0, \alpha_{p} \in \mathfrak{h}
$$

and

$$
\operatorname{deg}\left(\left[\alpha_{p}\right]\right)=\operatorname{deg}\left(x_{p}\right)+\operatorname{deg}\left(y_{p}\right)>0,
$$

contradicting the presupposition.

3. Since the auxiliary Lie algebra $\tilde{\mathfrak{g}}(A)$ is defined via the relations given in 3 , these relations hold in its quotient Lie algebra $\mathfrak{g}(A):=\mathfrak{g}(A) / \mathfrak{r}$, as well. In particular, we have the relations

$$
\left[h, x_{i}\right]=\underbrace{\alpha_{i}(h)}_{\in \mathbb{C}} x_{i} \text { for all } i \in\{1, \ldots, n\}
$$

with the linearly independent root basis $\Pi=\left\{\alpha_{1}, \ldots, \alpha_{n}\right\} \subset \mathfrak{h}^{*}$.

4. Let now $h$ be an element of $\mathfrak{h}$ of positive degree from the last part of the proof. Then, the degree of the left hand side of equation (1.92) is strictly greater than that of the right hand side, violating the gradation, unless $\alpha_{i}(h)=0$. But since this is true for all $i \in\{1, \ldots, n\}$, then the $\alpha_{i}$ cannot be linearly independent. So $g$ is not a Kac-Moody algebra.

We immediately conclude

\footnotetext{
${ }^{5}$ Definitions 2 through 4 only require $A$ to be a complex square matrix of rank $l$, but $\mathfrak{g}(A)$ is only called a Kac-Moody algebra if $A$ is a generalized Cartan Matrix.

${ }^{6}$ See [Kac82][§1.5]
} 
Corollary 1.7.3. $\quad$ 1. $g$ and all its infinite-dimensional subalgebras are not Kac-Moody, since they contain elements of positive degree.

2. The only subalgebras of $\mathfrak{g}$ that are Kac-Moody are finite-dimensional and subalgebras of $\mathfrak{g}_{0}$

3. For $d \neq 3+1, \mathfrak{g}_{0} \cong \mathfrak{s} \mathfrak{D}(d)$ is simple, so in this case, it is the only Kac-Moody subalgebra of $\mathfrak{g}$.

4. For $d=3+1, \mathfrak{g}_{0} \cong \mathfrak{s} \mathfrak{D}(4) \cong \mathfrak{s l}_{2} \oplus \mathfrak{s l}_{2}$, so Kac-Moody subalgebras of $\mathfrak{g}$ are either isomorphic to $\mathfrak{s D}(4)$ or $\mathfrak{s l}_{2}$

One interesting question that remains open to speculation is whether the PohlmeyerRehren Lie algebra can be extended to a Kac-Moody Lie algebra. For this, elements of negative degree would have to be introduced, such that we can have $0 \neq\left[x_{i}, y_{i}\right] \in \mathfrak{h}$ (unlike in part 2 of the proof of theorem 1.7.2) and the other axioms of Kac-Moody algebras can be satisfied. 


\subsection{Related Lie algebras}

As we have seen in lemma 1.6.4 the Pohlmeyer-Rehren Lie bracket conserves the magnetic quantum number. By considering alternative metric tensors, we can construct other Lie brackets on the same underlying vector space that conserve further quantities.

Proposition 1.8.1. $\quad$ 1. For $k \in I_{d}$ (cf. equation (1.66) ) substituting

$$
g_{i, j}^{ \pm_{k}}:= \begin{cases}-\alpha & \text { if } i= \pm_{k}, j=\mp_{k} \\ 0 & \text { else }\end{cases}
$$

for $g$ into equation (1.54) yields a Lie bracket on ime (g as a vector space). Denote it as $[\cdot, \cdot]_{ \pm_{k}}$ and the Lie algebra as ${ }_{ \pm k} \mathrm{~g}$. For $d=3$, the $k$ can be suppressed in the notation.

2. Additionally, for $d=2 n+1$, substituting

$$
g_{i, j}^{0}:= \begin{cases}-\alpha & \text { if } i=j=0 \\ 0 & \text { else }\end{cases}
$$

for $g$ into equation (1.54) yields another Lie bracket on ime. Denote it as $[\cdot, \cdot]_{0}$ and the Lie algebra as ${ }_{0} \mathfrak{g}$.

3. For all $x, y \in \mathfrak{g}$,

$$
[x, y]=\sum_{k=0}^{n-1}[x, y]_{ \pm_{k}}
$$

if $d=2 n$ and

$$
[x, y]=[x, y]_{0}+\sum_{k=1}^{n}[x, y]_{ \pm_{k}}
$$

if $d=2 n+1$.

4. For all $k \in I_{d}$, the Lie algebras ${ }_{ \pm} \mathfrak{g}$ and ${ }_{0} \mathfrak{g}$ are graded by $l=$ word length -2 and magnetic quantum numbers $m_{k}$.

5. For all $k \in I_{d,}{ }_{ \pm k} \mathfrak{g}$ is graded by the number of $+_{i}$ (as well as the number of $-{ }_{i}$ ) for all $i \neq k$ and if $d=2 n+1$ the number of zeroes.

6. If $d=2 n+1,{ }_{0} g$ is graded by the number of $+_{i}$ (as well as the number of $-i$ ) for all $i$.

7. For all $k \in I_{d,}{ }_{ \pm k} \mathfrak{g}$ is graded by the (number of $\left.+_{k}\right)-1$ (as well as the (number of $\left.\left.-{ }_{k}\right)-1\right)$ ).

8. If $d=2 n+1,{ }_{0} g$ is graded by the (number of zeroes) -2 .

Proof. 1,2,4 can be proved exactly analogously to the equivalent statements regarding $\mathfrak{g} .3$ follows directly from the fact that $g=\sum_{k=0}^{n-1} g^{ \pm_{k}}$ for $d=2 n$ and $g=g^{0}+\sum_{k=1}^{n} g^{ \pm_{k}}$ for $d=2 n+1$.

The remaining statements are proved by considering that $g^{ \pm_{k}}$ and $g^{0}$ are chosen so that all respective coefficients of Euler elements that would violate the claim on the right hand side 
of equation (1.54) are zero: $[\cdot, \cdot]_{ \pm_{k}}$ is constructed so that the summands on the right hand side of (1.54) have the same letter content as both words on the left hand side combined except for one $t_{k}$ and $-_{k}$ fewer each, proving 5 and 7 . Similarly, for $[\cdot, \cdot]_{0}$, the letter content on the right hand side is the same as that of both words on the left hand side combined except for two zeroes fewer, which proves 6 and 8 .

As in every graded Lie algebra, the stratum defined by $l=0$ is a subalgebra that acts via the adjoint action on the entire Lie algebra (lemma 2.2.2). Therefore, it is interesting to consider these subalgebras a bit more.

Proposition 1.8.2. 1. ${ }_{ \pm k} \mathfrak{g}_{0}$ is solvable but not nilpotent.

2. ${ }_{0} \mathfrak{g}_{0}$ is nilpotent.

Proof. 1. The Lie bracket $[\cdot, \cdot]_{ \pm_{k}}$ reduces the numbers of $+_{k}$ and $-_{k}$ by one each. So $e\left(-{ }_{k}+{ }_{k}\right) \notin_{ \pm k} \mathfrak{g}_{0}{ }^{(1)}$ for all $k \in I_{d}$. This implies $\left[[w, x]_{ \pm_{k}},[y, z]_{ \pm_{k}}\right]_{ \pm_{k}}=0$ for all $w, x, y, z \in_{ \pm k} \mathfrak{g}_{0}$, because each of the inner brackets evaluates to zero or an Euler elements with at most one of the letters $+_{k},-{ }_{k}$ (adding the occurences). In other words, the derived series stabilizes at ${ }_{0} \mathfrak{g}_{0}{ }^{(2)}=0$.

The fact that ${ }_{ \pm k} \mathfrak{g}_{0}$ is not nilpotent can be proved by showing that $e\left(0 \pm_{k}\right) \in{ }_{ \pm_{k}} \mathfrak{g}_{0}^{i}$ by induction over $i$ along its lower central series, always choosing $e\left(-{ }_{k}+k\right)$ as the element of $\mathfrak{g}_{0}$ to adjoin.

2. The Lie bracket $[\cdot, \cdot]_{0}$ reduces the number of zeroes by two. So, any $[x, y]_{0}$ contains no zeroes or vanishes $\forall x, y \in{ }_{0} \mathfrak{g}_{0}$. Therefore $\left[[x, y]_{0}, z\right]_{0}=0 \forall x, y, z \in{ }_{0} \mathfrak{g}_{0}$, in other words the lower central series stabilizes at ${ }_{0} \mathfrak{g}_{0}{ }^{2}=0$.

In particular, this means that the strata ${ }_{ \pm_{k}} \mathfrak{g}_{0}$ and ${ }_{0} \mathfrak{g}_{0}$ are not isomorphic to the simple Lie algebra $\mathfrak{g}_{0}=\mathfrak{s v}(d, \mathbb{C})$, which in turn implies that ${ }_{ \pm_{k}} \mathfrak{g}$ and ${ }_{0} \mathfrak{g}$ are not $\mathfrak{s v}(d, \mathbb{C})$-modules.

Therefore, the Lie algebras defined in this chapter are not interesting in the same way that $\mathfrak{g}$ is, but using their Lie brackets can be a handy notation for the technique of ignoring those Euler-Lyndon words in the right hand side of a Lie bracket that have more zeroes or plusses and minusses than the elements inside it which can be traced back at least to [PR86]. Example of such applications can be found in the proof of proposition 3.4 .3 and in the calculations featured in appendix $\mathrm{C}$ 


\section{Chapter 2}

\section{Structure Theory of the Pohlmeyer-Rehren Lie Algebra}

\subsection{Subalgebras and ideals}

We begin our examination of the structure of the Pohlmeyer-Rehren Lie algebra with some easy observations about subalgebras and ideals that also provide an opportunity to introduce some notation to be used later.

Lemma 2.1.1 (subalgebras and ideals from gradations). The following subsets of $\mathrm{g}$ are subalgebras:

$$
\begin{gathered}
\bigoplus_{l=0} \mathfrak{g}, \\
\mathfrak{g}_{l} \text { with } r \in \mathbb{N}, \\
\bigoplus_{l \in r \mathbb{N}} \mathfrak{g}^{m} \text { with } r \in \mathbb{N}_{0} \quad i \in I_{d}, \\
\bigoplus_{m_{i} \in r \mathbb{Z}} \mathfrak{g}^{m} \text { with } r \in \mathbb{N}_{0} \quad i \in I_{d}, \\
\bigoplus_{m_{i} \geq r} \mathfrak{g}^{m} \text { with } r \in \mathbb{N}_{0} \quad i \in I_{d}, \\
\bigoplus_{m_{i} \leq-r} \mathfrak{g}_{l}^{m} \text { with } r \in \mathbb{N}_{0}, c, c_{i} \in \mathbb{Z} \forall i \in I_{d} .
\end{gathered}
$$

The following subsets of $\mathfrak{g}$ are not only subalgebras, but ideals:

$$
\mathfrak{g}_{\geq r}:=\bigoplus_{l \geq r} \mathfrak{g}_{l} \text { with } r \in \mathbb{N}
$$

Proof. All these assertions follow from the facts that degree and magnetic quantum numbers are additive and that the stratum $\mathfrak{g}_{-1}$ is central.

Using the fact that an intersection of any family of subalgebras of a Lie algebra is a subalgebra, we can construct further subalgebras of $\mathfrak{g}$ from the ones given in the above lemma. 
An ideal that exists in any Lie algebra is its center.

Definition 2.1.2 (center). Let $G$ be a Lie algebra. The center of $G$ is the ideal defined as

$$
Z(G):=\{z \in G \mid[z, g]=0 \text { for all } g \in G\} .
$$

Recall that for one-letter words $x \in X$, we have $[e(x), g]=0$ for all $g \in \mathfrak{g}$ by the definition of the Lie bracket (1.54). Are there other central elements?

Conjecture 2.1.3. The center of $\mathfrak{g}$ is $\mathfrak{g}_{-1}$.

Using the Mathematica module CenterTest (B.6.8), it was verified that there are no central elements in $\bigoplus_{l=1}^{8} \mathfrak{g}_{l}$ for $d=3$ and $d=4$. Note that there are some linearly independent elements $g, h \in \mathfrak{g}_{\geq 1}$ with $[g, h]=0$. The set of all such elements $g$ for a given $h$ can be calculated as ker $\operatorname{ad}_{h}$ using the Mathematica module Kernel.

Lemma 2.1.4 (subalgebras from restrictions of the alphabet). Let $X^{\prime \prime} \subset X^{\prime} \subset X$ and $r \in \mathbb{N}_{0}$. Then, the linear span of the elements

$$
e\left(x_{1}, \ldots, x_{n}\right)
$$

with $x_{i} \in X^{\prime}$ for all $i \in\{1, \ldots, n\}$, all $n \in \mathbb{N}_{+}$and $x_{1}, \ldots, x_{r}, x_{n-r+1}, \ldots, x_{n} \in X^{\prime \prime}$ constitute a Lie subalgebra of $\mathfrak{g}$.

Proof. Follows directly from formula (1.54) for the Lie bracket on $g$. Consider the Lie bracket of two basis elements $e\left(x_{1} \ldots x_{n}\right), e\left(y_{1} \ldots y_{m}\right)$. If $n=1$ or $m=1$, the statement is trivial. Otherwise, the Lie bracket is

$$
\left[e\left(x_{1} \ldots x_{n}\right), e\left(y_{1} \ldots y_{m}\right)\right]=\sum_{i=1}^{n} \sum_{j=1}^{m}(-1)^{n+i+j+1} g_{x_{i}, y_{j}} e\left(\begin{array}{l|l|l}
x_{1} \ldots x_{i-1} & y_{j-1} \ldots y_{1} \\
x_{n} \ldots x_{i+1} & y_{j+1} \ldots y_{m}
\end{array}\right) .
$$

Obviously, all words occurring on the right hand side are permutations of subwords of $x_{1} \ldots x_{n} y_{1} \ldots y_{m}$ (each with two letters removed), so their letters are all in $X^{\prime}$. By iteratively using the recursive formula for the shuffle product (lemma 1.1.4) $r$ times, we find that the first $r$ letters of the words occuring on the right hand side can only be elements of the set $\left\{x_{1}, \ldots, x_{r}, x_{n-r+1}, \ldots, x_{n}\right\} \subset X^{\prime \prime}$. Analogously, the last $r$ letters of the words occurring on the right hand side can only be elements of $\left\{y_{r}, \ldots, y_{1}, y_{m-r+1}, \ldots, y_{m}\right\} \subset X^{\prime \prime}$

Remark 2.1.5. Note that the elements described above are not Euler-Lyndon elements in general. The special case $X^{\prime \prime}=X \backslash\{0\}, X^{\prime}=X, r=1$ was used in [PR86, p. 622] to prove the fact (accounted of herein as proposition 3.2.7) that some elements of the Poisson algebra of invariants of the Nambu-Goto string are exceptional (cf. chapter 3). An alternative way to prove the fact that this is a subalgebra is that it can be written as

$$
\operatorname{ker} \partial_{0}^{R} \cap \operatorname{ker} \partial_{0}^{L}=\operatorname{span}\left(x \in X^{*} \text { with } x=1_{\emptyset} \text { or } x_{1}=x_{n}=0\right)
$$

and kernels of derivations and the intersections of other subalgebras always produce subalgebras.

Remark 2.1.6. Note that the list of subalgebras and ideals given in this section is not exhaustive. Some results regarding abelian subalgebras of $\mathfrak{g}$ including infinite-dimensional abelian subalgebras that are not Cartan subalgebras will be given in 2.3.18. 


\subsection{Structure of the subalgebra $\mathfrak{g}_{0}$}

The remainder of this section will primarily use representation theory to obtain some information on the Pohlmeyer-Rehren Lie algebra. We begin by recounting some basic definitions establishing the closely related notions of modules and representations for easy reference:

Definition 2.2.1. (commutator, general linear Lie algebra, representation, module, action, homo-/epi-/mono-/isomorphism of modules, adjoint representation, direct sum of modules semidirect product, reducible, irreducible)

1. Let $V$ be a vector space over a field $\mathbb{K}$. Then the general linear group GL $(V)$ together with the commutator $[\cdot, \cdot]: \mathrm{GL}(V) \times \mathrm{GL}(V) \rightarrow \mathrm{GL}(V)$,

$$
[A, B]:=A B-B A \text { for all } A, B \in \mathrm{GL}(V)
$$

is a Lie algebra over $\mathbb{K}$, designated as the general linear Lie algebra $\operatorname{gl}(V)$.

2. Let $G$ be a Lie algebra over a field $\mathbb{K}$ and let $V$ be a $\mathbb{K}$-vector space. A homomorphism of Lie algebras $\rho: G \rightarrow \operatorname{gl}(V)$ is then called a representation of $G$; equivalently $V$ is called a $G$-module. We call $g \cdot v:=\rho(g)(v)$ for all $g \in G, v \in V$ the action of $G$ on $V$.

3. Let $G$ be a Lie algebra and let $V, W$ be $G$-modules via the representations $\rho: G \rightarrow \mathfrak{g l}(V)$, $\sigma: G \rightarrow \mathfrak{g l}(W)$. A homomorphism / epimorphism / monomorphism / isomorphism of vector spaces $\phi: V \rightarrow W$ is called a homomorphism / epimorphism / monomorphism / isomorphism of G-modules if

$$
\phi(\rho(g)(v)):=\sigma(g)(\phi(v)) \text { for all } g \in G, v \in V .
$$

If an isomorphism of $G$-modules between two $G$-modules $V$ and $W$ exists, then $V$ and $W$ are called isomorphic and their representations are called equivalent.

4. Let $G$ be a Lie algebra. Then $G$ is a $G$-module via the adjoint representation ad : $G \rightarrow \mathfrak{g l}(G)$,

$$
\operatorname{ad}_{g}\left(g^{\prime}\right):=\left[g, g^{\prime}\right] \text { for all } g, g^{\prime} \in G .
$$

5. Let $G$ be a Lie algebra and let $V, W$ be $G$-modules via the representations $\rho: G \rightarrow \mathfrak{g l}(V)$, $\sigma: G \rightarrow \mathfrak{g l}(W)$. Then $V \oplus W$ (direct sum of vector spaces) with the action

$$
g .(v, w):=(\rho(g)(v), \sigma(g)(w)) \text { for all } g \in G, v \in V, w \in W
$$

is a $G$-module called the direct sum $V \oplus W$.

6. Let $\left(G,[\cdot, \cdot]_{G}\right)$ and $(H,[\cdot, \cdot])$ be Lie algebras such that $H$ is a $G$-module via the representation $\rho: G \rightarrow \mathfrak{g l}(H)$. Then $H \oplus G$ (direct sum of vector spaces) with the Lie bracket $[\cdot, \cdot]: G \oplus H \times G \oplus H \rightarrow G \oplus H$ defined by

$$
\left[(h, g),\left(h^{\prime}, g^{\prime}\right)\right]:=\left(\left[h, h^{\prime}\right]_{H}+\rho(g)\left(h^{\prime}\right)-\rho\left(g^{\prime}\right)(h),\left[g, g^{\prime}\right]_{G}\right) \text { for all } h, h^{\prime} \in H, g, g^{\prime} \in G
$$

is a Lie algebra called the semidirect product $H \rtimes_{\rho} G$. Note that any vector space $V$ that is a $G$-module can be used as $H$ in this construction since $V$ is a Lie algebra with the trivial Lie bracket 0 . 
7. Let $G$ be a Lie algebra and let $V$ be a $G$-module via the representation $\rho: G \rightarrow \operatorname{gl}(V)$. Then $V$ and $\rho$ are called reducible if $V$ can be written as a direct sum of nonzero Gmodules; otherwise, $V$ and $\rho$ are called irreducible.

The following simple general fact about $\mathbb{Z}$-graded Lie algebras shows us the significance of the stratum $\mathfrak{g}_{0}$ for the Pohlmeyer-Rehren Lie algebra.

Lemma 2.2.2 (graded Lie algebra as a module of its 0-th stratum). Let $G$ be a $\mathbb{Z}$-graded Lie algebra, i.e. $G=\bigoplus_{l \in \mathbb{Z}} G_{l}$ and $\left[G_{l}, G_{l^{\prime}}\right] \subset G_{l+l^{\prime}}$. Then:

1. $G_{0}$ is a subalgebra of $G$.

2. For all $l \in \mathbb{Z}, G_{l}$ is a $G_{0}$-module (via the adjoint action).

3. $G$ is a $G_{0}$-module (via the adjoint action).

Proof. 1. Follows directly from the additivity of the gradation with $l=l^{\prime}=0$.

2. Closedness directly follows from the additivity of the gradation using $l^{\prime}=0$. Everything else is a consequence of the fact that $G$ is a Lie algebra.

3. A direct sum of $G_{0}$-modules is a $G_{0}$-module.

\subsection{1 $\mathfrak{g}_{0}$ is isomorphic to $\mathfrak{s} \mathfrak{d}(d, \mathbb{C})$}

Lemma 2.2.3. As a Lie algebra, $\mathfrak{g}_{0}$ is isomorphic to a special orthogonal Lie algebra:

$$
\mathfrak{g}_{0} \cong \mathfrak{s o}(d, \mathbb{C}):=\left\{M \in \operatorname{Mat}(d \times d) \mid M^{t}+M=0\right\} .
$$

Proof. A basis of $\mathfrak{s p}(d, \mathbb{C})$ is given by $b_{i j}=E_{i j}-E_{j i}$, with $1 \leq i<j \leq d$, where $E_{i j}$ is the $d \times d$ matrix with a 1 in position $(i, j)$ and 0 everywhere else. The Lie bracket on these elements is

$$
\left[b_{i j}, b_{k l}\right]=\delta_{j k} b_{i l}-\delta_{j l} b_{i k}-\delta_{i k} b_{j l}+\delta_{i l} b_{j k} .
$$

Define the linear map

$$
\varphi: \mathfrak{s o}(d, \mathbb{C}) \rightarrow \mathfrak{g}_{0}, \quad b_{i j} \mapsto \frac{1}{\alpha} \mathbf{i}^{\delta_{i 1}} e(i-1 j-1) .
$$

Since it maps a basis to a basis, $\varphi$ is an isomorphism of vector spaces. It is also an isomorphism of Lie algebras:

$$
\begin{aligned}
\varphi\left(\left[b_{i+1 j+1}, b_{k+1 l+1}\right]_{\mathfrak{s o}(d, \mathbb{C})}\right)= & \varphi\left(\delta_{j k} b_{i+1 l+1}-\delta_{j l} b_{i+1 k+1}-\delta_{i k} b_{j+1 l+1}+\delta_{i l} b_{j+1 k+1}\right) \\
= & \frac{1}{\alpha}\left(\delta_{j k} \mathbf{i}^{\delta_{i 0}} e(i l)-\delta_{j l} \mathbf{i}^{\delta_{i 0}} e(i k)-\delta_{i k} \mathbf{i}^{\delta_{j 0}} e(j l)+\delta_{i l} \mathbf{i}^{j 0} e(j k)\right) \\
\stackrel{*}{=} & \frac{1}{\alpha}\left(\mathbf{i}^{\delta_{i 0}+\delta_{k 0}+\delta_{j 0}+\delta_{k 0}} \delta_{j k} e(i l)-\mathbf{i}^{\delta_{i 0}+\delta_{k 0}+\delta_{j 0}+\delta_{l 0}} \delta_{j l} e(i k)\right. \\
& \left.-\mathbf{i}^{\delta_{i 0}+\delta_{k 0}+\delta_{j 0}+\delta_{k 0}} \delta_{i k} e(j l)+\mathbf{i}^{\delta_{i 0}+\delta_{k 0}+\delta_{i 0}+\delta_{l 0}} \delta_{i l} e(j k)\right) \\
\stackrel{* *}{=} & \frac{1}{\alpha^{2}} \mathbf{i}^{\delta_{i 0}+\delta_{k 0}}\left(g_{j k} e(i l)-g_{j l} e(i k)-g_{i k} e(j l)+g_{i l} e(j k)\right) \\
\stackrel{1.54}{=} & \frac{1}{\alpha^{2}} \mathbf{i}^{\delta_{i 0}+\delta_{k 0}}[e(i j), e(k l)]_{\mathrm{g}_{0}} \\
= & {\left[\varphi\left(b_{i+1} j+1\right), \varphi\left(b_{k+1 l+1}\right)\right]_{\mathfrak{g}_{0}}, }
\end{aligned}
$$


where in (*) the fact that $0 \leq i<j, 0 \leq k<l \Rightarrow \delta_{j 0}=\delta_{k 0}=0$ is used; in (**) we use the following alternative description of the Minkowski metric:

$$
g_{a b}=\alpha \delta_{a b} \mathbf{i}^{\delta_{a 0}+\delta_{b 0}}
$$

Remark 2.2.4. Note that the inverse isomorphism $\mathfrak{g}_{0} \rightarrow \mathfrak{s v}(d, \mathbb{C})$ is given by

$$
g_{i j} \mapsto \alpha(-\mathbf{i})^{\delta_{i 0}} b_{i+1} j+1 .
$$

The Lie algebras $\mathfrak{s}(d, \mathbb{C})$ are among the classical Lie algebras whose structure and representations are thoroughly explored, and the above isomorphism allows to immediately transfer this information to $\mathfrak{g}_{0}$

Corollary 2.2.5. $\quad$ 1. $\mathfrak{g}_{0}$ is a semisimple Lie algebra.

2. For $d \neq 4, \mathrm{~g}_{0}$ is a simple Lie algebra (i.e. it has no proper ideals).

Proof. The above statements hold for $\mathfrak{s} \mathfrak{D}(d, \mathbb{C})$.

In the same vein, all the structure of $\mathfrak{g}_{0}$ including the statements in the remainder of this and the following section can be recovered using lemma 2.2.3 (see [Hei90][p. 229ff] for a concise account of the root space decomposition of all the classical simple Lie algebras), but since we have an opportunity to see directly how the structure theory arises from the way that Lie brackets of Euler-Lyndon words are formed from contracting corresponding plusses and minusses (equation (1.54)), it is instructive to develop the root space decomposition of finitedimensional semisimple Lie algebras directly, i.e. without making use of the isomorphism from lemma 2.2.3. Having the root space decomposition in these concrete terms is also useful for the subsequent task of decomposing the entire Lie algebra $g$ by weight spaces in section 2.3. Throughout the remainder of this section, we will recount some standard theory, taking definitions from the account of [Hei90][p 223f.] and develop the application to $\mathfrak{g}_{0}$.

\subsubsection{Cartan subalgebra of $\mathfrak{g}_{0}$}

Since Cartan subalgebras play a key role in the theory of Lie algebras, it is useful to ask if we can identify them.

Definition 2.2.6 (Cartan subalgebra). Let $G$ be a Lie algebra. A Cartan subalgebra of $G$ is a subalgebra $H$ of $G$ that is nilpotent and is its own normalizer in $G$, i.e. satisfies

$$
[g, h] \in H \text { for all } h \in H \Rightarrow g \in H .
$$

The Cartan subalgebras of the subalgebras $\mathfrak{g}_{0} \cong \mathfrak{s v}(d)$ are well known in the literature.

Proposition 2.2.7. The basis vectors

$$
\tilde{h}_{i}:=e\left(-{ }_{i}+{ }_{i}\right) \text { for } i \in I_{d}
$$

span a Cartan subalgebra of $\mathfrak{g}_{0}$ that we will designat ${ }^{1} \mathfrak{H}$.

\footnotetext{
${ }^{1}$ We use an uppercase $\mathfrak{H}$ to distinguish this Cartan subalgebra in notation from the Poisson algebra of invariant charges $\mathfrak{h}$ that will be introduced later in $\mathrm{thm}$. 3.2 .3
} 
Proof. We obtain both nilpotency and self-normalization as a consequence of lemma 1.6.5. by that lemma, all Euler-Lyndon elements in $\mathfrak{g}_{0}$ (which form a basis of $\mathfrak{g}_{0}$ ) satisfy the equation with $\left[\tilde{h}_{i}, x\right]=m_{i} x$. The right hand side is zero for all $i$ if and only if all $m_{i}$ are zero, in other words if all the words in $x$ contain the same numbers of corresponding plusses and minusses - since we only consider the case of $l=0$, this is only possible for the $\tilde{h}_{j}$.

Remark 2.2.8. The preceding proposition can alternatively be proved by considering the root system to be introduced in remark 2.2.16 and applying SERRE's theorem [Hum72, section 18.3].

While $\mathfrak{H}$ is a Cartan subalgebra of $\mathfrak{g}_{0}, \mathfrak{H}$ is not a Cartan subalgebra of the whole of $\mathfrak{g}$ because $\mathfrak{H}$ no longer is self-normalizing (as subalgebras of $\mathfrak{g}$ instead of $\mathfrak{g}_{0}$ ). Consider the example case $d=3$. Here, $\mathfrak{H}=\mathbb{C} \cdot e(-+)$, and the entire subalgebra $\mathfrak{g}^{0}$ commutes with this element.

But is $\mathfrak{g}^{0}$ (defined by $m=0$, cf. 1.6.4), a subalgebra of which is $\mathfrak{H}$, a Cartan subalgebra of $\mathfrak{g}$ ? No, because $\mathfrak{g}^{0}$ is not nilpotent, as the following result shows.

Proposition 2.2.9. For $d \geq 3$, every subalgebra of $\mathfrak{g}^{0}$ that contains the elements of $\mathfrak{g}_{1}^{0}$ is infinitedimensional and non-nilpotent.

Proof. $\quad$ 1. For every $l \geq 0$, the Euler-Lyndon word $e\left(-0^{l}+\right)$ is an element of $\mathfrak{g}^{0}$. Since they are all of different degrees, these infinitely many elements are linearly independent, so $\mathrm{g}^{0}$ is infinite-dimensional.

2. To show that $\mathfrak{g}^{0}$ is not nilpotent, we will show that the element $e(0-+) \in \mathfrak{g}_{1}^{0}$ is not adnilpotent. With the abbreviation $Z_{l}:=$ \{words of $l+3$ letters with fewer than $l+1$ zeroes $\}$, we calculate

$$
\begin{aligned}
& \operatorname{ad}_{e(0-+)} e\left(-0^{l}+\right) \\
= & -\left[e\left(-0^{l}+\right), e(0-+)\right] \\
= & -((-1)^{l+2+l+2+2} \alpha e\left(-0^{l} \begin{array}{l}
0 \\
+
\end{array}\right)+(-1)^{l+2+1+3} \alpha \underbrace{\frac{1.2 .5}{-}(-1)^{l+1} e\left(-\begin{array}{c}
0 \\
0^{l+}
\end{array}\right)} \underbrace{e\left(+0^{l}-0\right)}_{w \in Z_{l}})+\sum_{w} c_{w} e(w) \\
= & -\alpha\left(e\left(-0^{l+1}+\right)+e\left(-0^{l}+0\right)-e\left(-0^{l}+0\right)-(l+1) e\left(-0^{l+1}+\right)\right)+\sum_{w \in Z_{l}} c_{w} e(w) \\
= & \left.\alpha l e\left(-0^{l+1}+\right)\right)+\sum_{w \in Z_{l}} c_{w} e(w)
\end{aligned}
$$

with some coefficients $c_{w} \in \mathbb{C} \forall w \in Z_{l}$. Because the words on the right hand side of the defining equation (1.54) of the Lie bracket never contain more instances of a particular letter than the words on the left hand side in total, this implies by induction:

$$
\left.\operatorname{ad}_{e(0-+)}^{n} e(-0+)=\alpha^{n} n ! e\left(-0^{n+1}+\right)\right)+\sum_{w \in Z_{n}} d_{w} e(w) \neq 0
$$

with some coefficients $d_{w} \in \mathbb{C} \forall w \in Z_{n}$. Since the elements of $\mathfrak{g}$ on the left-hand side of the above equation are both elements of $\mathfrak{g}_{1}^{0}$, the equation also shows that no subalgebra of $\mathfrak{g}$ containing $\mathfrak{g}_{1}^{0}$ can be nilpotent or finite-dimensional $:^{2}$

In fact, these two elements actually span the two-dimensional vector space $\mathfrak{g}_{0}^{1}$. 


\subsubsection{General outline of the root space decomposition}

Given a Cartan subalgebra, we can define roots and their corresponding root spaces.

Definition 2.2.10 (root, root space, base, simple root, positive root, negative root, $>$ ).

1. Let $G$ be a finite-dimensional semisimple Lie algebra with Cartan subalgebra $H$ and a linear form $\alpha \in H^{*}$. Define

$$
G^{\alpha}:=\{g \in G \mid[h, g]=\alpha(h) g \forall h \in H\} .
$$

If $G^{\alpha} \neq 0$ and $\alpha \neq 0$, then $\alpha$ is called a root of $G$, and $G^{\alpha}$ is called its root space. We designate the set of all roots by $R$.

2. A subset $B \subset R$ is called a base of $R$ if $B$ is $\mathbb{R}$-linearly independent and every root $\alpha \in R$ can be written as

$$
\alpha= \pm \sum_{\beta \in B} m_{\beta} \beta
$$

with coefficients $m_{\beta} \in \mathbb{N}_{0}$. The elements of $B$ are called simple roots; those roots with a plus (minus) sign on the right hand sign of the above equation are called positive (negative) roots, and the sets of positive (negative) roots are designated by $R^{ \pm}$.

3. If $B$ is a base, we finally define the partial order $>$ on $H^{*}$ by

$$
\alpha>\beta: \Leftrightarrow \alpha-\beta=\sum_{\gamma \in B} m_{\gamma} \gamma
$$

for $\alpha, \beta \in H^{*}$ with coefficients $m_{\gamma} \in \mathbb{N}_{0}$.

Among the key results in the theory of finite-dimensional semisimple Lie algebras are the fact that bases always exist and the so-called root space decomposition that can now be formulated using the notions just introduced:

Theorem 2.2.11 (root space decomposition). Under the preconditions of the above definition 2.2.10. we can decompose the Lie algebra $G$ into a direct sum:

$$
G=H \oplus \bigoplus_{\alpha \in R} G^{\alpha}
$$

Proof. See [Hei90, p. 223f].

There are symmetries of the root system enforced by the Weyl group:

Definition 2.2.12 (Weyl group). The Weyl group $\mathcal{W}$ is the subgroup of $\mathrm{GL}\left(H^{*}\right)$ generated by the reflections $\sigma_{\alpha}, \alpha \in R$,

$$
\sigma_{\alpha}(\beta):=\beta-\frac{2(\beta, \alpha)}{(\alpha, \alpha)} \alpha
$$

for all $\beta \in H^{*}$. 
The scalar product $(\cdot, \cdot)$ of roots is defined the following way: since $G$ is semisimple, the restriction of the Killing form $\kappa: G \times G \rightarrow \mathbb{K}$,

$$
\kappa(x, y):=\operatorname{trace}\left(\operatorname{ad}_{x} \circ \operatorname{ad}_{y}\right) \text { for all } x, y \in G
$$

to the Cartan subalgebra $H$ is nondegenerate, so for any $\varphi \in H^{*}$ we can find a unique $t_{\varphi} \in H$ such that $\varphi(h)=\kappa\left(t_{\varphi}, h\right)$ holds for all $h \in H$. Then define the scalar product $(\cdot, \cdot): H^{*} \times H^{*} \rightarrow \mathbb{C}$,

$$
(\alpha, \beta):=\kappa\left(t_{\alpha}, t_{\beta}\right)
$$

for $\alpha, \beta \in H^{*}$.

Theorem 2.2.13 (properties of the Weyl group). Let $B$ be a base of the root system $R$ and let $W$ be the corresponding Weyl group.

1. If $B^{\prime}$ is another base of $R$, then there is a $\sigma \in \mathcal{W}$ such that $\sigma\left(B^{\prime}\right)=B$.

2. For any $\alpha \in R$, there is $a \sigma \in \mathcal{W}$ such that $\sigma(\alpha) \in B$.

3. $\mathcal{W}$ is generated by $\left\{\sigma_{\beta} \mid \beta \in B\right\}$.

Proof. See [Hum72, Thm. 10.3]

\subsubsection{Root space decomposition of $\mathfrak{g}_{0}$}

Let us now return from the general theory to the special case of the stratum $\mathfrak{g}_{0}$ of the Pohlmeyer-Rehren Lie algebra $\mathfrak{g}$. Since we are only considering the subalgebra $\mathfrak{g}_{0}$, we are in the finite-dimensional case, and a (linear) basis of the dual space $\mathfrak{H}^{*}$ is given by the dual basis vectors $\gamma_{i}, i \in I_{d}$ of $\mathfrak{H}$ characterized by

$$
\gamma_{i}\left(\tilde{h}_{j}\right)=\delta_{i j}
$$

With this basis in mind, we can immediately use lemma 1.6 .5 to find the roots of $\mathfrak{g}_{0}$. 
Theorem 2.2.14 (roots and root spaces of $\mathfrak{g}_{0}$ ).

1. If $d$ is even, the roots of $\mathrm{g}_{0}$ are

$$
R=\left\{a \gamma_{i}+b \gamma_{j} \mid a, b= \pm 1, i \neq j, i, j \in I_{d}\right\} .
$$

The corresponding root spaces are

$$
\begin{aligned}
\mathfrak{g}_{0}^{\gamma_{i}+\gamma_{j}} & =\left\langle e\left(+_{i}+{ }_{j}\right)\right\rangle, \\
\mathfrak{g}_{0}^{-\gamma_{i}+\gamma_{j}} & =\left\langle e\left(-_{i}+{ }_{j}\right)\right\rangle, \\
\mathfrak{g}_{0}^{-\gamma_{i}-\gamma_{j}} & =\left\langle e\left(-_{i}-{ }_{j}\right)\right\rangle .
\end{aligned}
$$

A set of positive roots is given by

$$
R^{+}=\left\{ \pm \gamma_{i}+\gamma_{j} \mid i<j, i, j \in I_{d}\right\}
$$

and a base by

$$
\begin{aligned}
\alpha_{0} & :=\gamma_{0}+\gamma_{1}, \\
\alpha_{i} & :=-\gamma_{i-1}+\gamma_{i} \text { for } i \in I_{d} \backslash\{0\} .
\end{aligned}
$$

2. If $d$ is odd, the roots of $\mathfrak{g}_{0}$ are

$$
R=\left\{a \gamma_{i}+b \gamma_{j} \mid a= \pm 1, b \in\{0, \pm 1\}, i \neq j, i, j \in I_{d}\right\} .
$$

The corresponding root spaces are

$$
\begin{aligned}
\mathfrak{g}_{0}^{\gamma_{i}} & =\left\langle e\left(0+_{i}\right)\right\rangle, \\
\mathfrak{g}_{0}^{-\gamma_{i}} & =\left\langle e\left(0-{ }_{i}\right)\right\rangle, \\
\mathfrak{g}_{0}^{\gamma_{i}+\gamma_{j}} & =\left\langle e\left(+_{i}+_{j}\right)\right\rangle, \\
\mathfrak{g}_{0}^{-\gamma_{i}+\gamma_{j}} & =\left\langle e\left(-_{i}+_{j}\right)\right\rangle, \\
\mathfrak{g}_{0}^{-\gamma_{i}-\gamma_{j}} & =\left\langle e\left(-_{i}{ }_{j}\right)\right\rangle .
\end{aligned}
$$

A set of positive roots is given by

$$
\begin{aligned}
R^{+}= & \left\{\gamma_{i} \mid i \in I_{d}\right\} \\
\cup & \left\{ \pm \gamma_{i}+\gamma_{j} \mid i<j, i, j \in I_{d}\right\},
\end{aligned}
$$

and a base by

$$
\begin{aligned}
\alpha_{1} & :=\gamma_{1} \\
\alpha_{i} & :=-\gamma_{i-1}+\gamma_{i} \text { for } i \in I_{d} \backslash\{1\} .
\end{aligned}
$$


Proof. - We begin by showing that the linear forms given above are actually roots and simultaneously calculating their root spaces. Since the $\tilde{h}_{k}$ with $k \in I_{d}$ defined in 1.6.5 are a basis of the Cartan subalgebra $\mathfrak{H}$ and due to linearity, the condition defining a root from equation (2.21) can be reformulated to

$$
\mathfrak{g}^{\alpha}=\left\{g \in \mathfrak{g} \mid\left[\tilde{h}_{k}, g\right]=\alpha\left(\tilde{h}_{k}\right) g \forall k \in I_{d}\right\} .
$$

We can now make use of the fact that we already calculated the Lie brackets of the elements of the Cartan basis and the other Euler-Lyndon elements in lemma 1.6.5. Since all calculations are very similar, we only consider two examples, first for even $d$ :

$$
\begin{aligned}
& \mathfrak{g}_{0}^{\gamma_{i}+\gamma_{j}}=\left.\left\{g \in \mathfrak{g}_{0} \mid\left[\tilde{h}_{k}, g\right]=\left(\gamma_{i}+\gamma_{j}\right)\left(\tilde{h}_{k}\right)\right] g \forall k \in I_{d}\right\} \\
& \stackrel{\text { 2.2.4 }}{=}\left\{g \in \mathfrak{g}_{0} \mid\left[\tilde{h}_{k}, g\right]=\left(\delta_{i k}+\delta_{j k}\right) g \forall k \in I_{d}\right\} \\
& \stackrel{1.6 .5}{=}\left\{c \cdot e\left(x_{1} x_{2}\right) \mid m_{k}\left(e\left(x_{1} x_{2}\right)\right)=\left(\delta_{i k}+\delta_{j k}\right) \forall k \in I_{d}, c \in \mathbb{C}, x_{1}, x_{2} \in X_{ \pm}\right\} \\
& \stackrel{1.6 .3}{=}\left\langle e\left(++_{i}+{ }_{j}\right)\right\rangle .
\end{aligned}
$$

The calculations for the roots $\gamma_{i}-\gamma_{j}$ and $-\gamma_{i}-\gamma_{j}$ are analogous. If $d$ is odd, we also encounter the slightly different calculation

$$
\begin{aligned}
& \mathfrak{g}_{0}^{\gamma_{i}}=\ldots \\
& \quad=\left\{c \cdot e\left(x_{1} x_{2}\right) \mid m_{k}\left(e\left(x_{1} x_{2}\right)\right)=\delta_{i k} \forall k \in I_{d}, c \in \mathbb{C}, x_{1}, x_{2} \in X_{ \pm}\right\} \\
& \stackrel{1.6 .3}{=}\left\langle e\left(0+{ }_{i}\right)\right\rangle,
\end{aligned}
$$

and, analogously, $\mathfrak{g}_{0}^{\gamma_{i}}=\left\langle e\left(0+{ }_{i}\right)\right\rangle$. Calculations of this type show that the linear forms are roots and their root spaces are as indicated. Since we can establish an obvious bijection between the root spaces and the Euler-Lyndon elements, and the latter are a linear basis of $\mathfrak{g}_{0}$ by theorem 1.3.4. the direct sum of $\mathfrak{H}$ and the root spaces is (all of) $\mathfrak{g}_{0}$. A consequence from this and theorem 2.2.11 is that there can be no other root.

- It is obvious that $R= \pm R^{+}$.

- To show that the linear forms $\alpha_{i}, \alpha_{i}$ given are actually simple roots, we represent all positive roots as sums of them. Recall $i, j \in I_{d}$ and $i<j$. If $d$ is even,

$$
\begin{aligned}
-\gamma_{i}+\gamma_{j} & =\sum_{k=i+1}^{j} \alpha_{k}, \\
\gamma_{i}+\gamma_{j} & =\alpha_{0}+\sum_{k=1}^{i} \alpha_{k}+\sum_{k=1}^{j} \alpha_{k} .
\end{aligned}
$$

If $d$ is odd,

$$
\begin{aligned}
\gamma_{i} & =\alpha_{1}+\sum_{k=2}^{i} \alpha_{k}, \\
\gamma_{i}+\gamma_{j} & =2 \alpha_{1}+\sum_{k=2}^{i} \alpha_{k}+\sum_{k=2}^{j} \alpha_{k}, \\
-\gamma_{i}+\gamma_{j} & =\sum_{k=i+1}^{j} \alpha_{k} .
\end{aligned}
$$


Remark 2.2.15. At the beginning of this section, we proved that $\mathfrak{g}_{0}$ is isomorphic to $\mathfrak{s}(d, \mathbb{C})$ by directly giving an isomorphism. The same statement can alternatively be proved using DYNKIN's celebrated classification of the finite-dimensional simple Lie algebras by their root systems and the fact that theorem 2.2.14 describes the root system of $\mathfrak{g}_{0}$. These root systems are the following:

- For $d=2, \mathrm{~g}_{0} \cong \mathfrak{s} \mathfrak{D}(2, \mathbb{C})$ is one-dimensional, hence abelian.

- For $d=3, \mathfrak{g}_{0} \cong \mathfrak{s} \mathfrak{0}(3, \mathbb{C}) \cong \mathfrak{s l}(2)$ has root system type $A_{1}$.

- For $d=4, \mathfrak{g}_{0} \cong \mathfrak{s v}(4, \mathbb{C}) \cong \mathfrak{s l}(2) \times \mathfrak{s l}(2)$ has root system type $A_{1} \times A_{1}$.

- For $d=2 n+1, n \geq 2, \mathfrak{g}_{0} \cong \mathfrak{s} \mathfrak{v}(d, \mathbb{C})$ has root system type $B_{n}$.

- For $d=2 n, n \geq 3, \mathfrak{g}_{0} \cong \mathfrak{s} \mathfrak{0}(d, \mathbb{C})$ has root system type $D_{n}$.

Remark 2.2.16. For any semisimple finite-dimensional complex Lie algebra $G$ with Cartan subalgebra $H$ and set of roots $R$, one can find elements $h_{\alpha} \in H, x_{\alpha} \in G^{\alpha}$, such that

$$
\begin{aligned}
\alpha\left(h_{\alpha}\right) & =2, \\
{\left[x_{\alpha}, x_{-\alpha}\right] } & =h_{\alpha}
\end{aligned}
$$

for all $\alpha \in R$. In particular, for a base $\left(\alpha_{i}\right)_{i \in I}$, with index set $I$ we can define

$$
h_{i}:=h_{\alpha_{i}}, x_{i}:=x_{\alpha_{i}}, y_{i}:=x_{-\alpha_{i}}
$$

(cf. [Hei90, p. 235]). In case of $\mathfrak{g}_{0}$, such $h_{i}, x_{i}, y_{i}$ are given by

1. if $d$ is even,

$$
\begin{aligned}
h_{0} & :=\frac{1}{\alpha}\left(e\left(--_{0}+0\right)+e\left(-{ }_{1}+1\right)\right), \\
h_{i} & :=\frac{1}{\alpha}\left(-e\left(-_{i-1}+{ }_{i-1}\right)+e\left(-{ }_{i}+{ }_{i}\right)\right) \text { for } i \in I_{d} \backslash\{0\}, \\
x_{0} & :=\frac{1}{\alpha} e\left(+_{0}+1\right), \\
x_{i} & :=\frac{1}{\alpha} e\left(-{ }_{i-1}+{ }_{i}\right) \text { for } i \in I_{d} \backslash\{0\}, \\
y_{0} & :=-\frac{1}{\alpha} e(-0-1), \\
y_{i} & :=-\frac{1}{\alpha} e\left(+_{i-1}-i\right) \text { for } i \in I_{d} \backslash\{0\},
\end{aligned}
$$


2. if $d$ is odd,

$$
\begin{aligned}
h_{1} & :=\frac{2}{\alpha} e(-1+1), \\
h_{i} & :=\frac{1}{\alpha}\left(-e\left(-{ }_{i-1}+{ }_{i-1}\right)+e\left(-{ }_{i}+{ }_{i}\right)\right) \text { for } i \in I_{d} \backslash\{1\}, \\
x_{1} & :=\frac{\sqrt{2}}{\alpha} e\left(0++_{1}\right), \\
x_{i} & :=\frac{1}{\alpha} e\left(-{ }_{i-1}+{ }_{i}\right) \text { for } i \in I_{d} \backslash\{1\}, \\
y_{1} & :=\frac{\sqrt{2}}{\alpha} e\left(0--_{1}\right), \\
y_{i} & :=-\frac{1}{\alpha} e\left(+_{i-1}-{ }_{i}\right) \text { for } i \in I_{d} \backslash\{1\} .
\end{aligned}
$$

The Weyl group can be given in a way well suited to the description of the spin basis:

Lemma 2.2.17. 1. For odd $d$, the Weyl group $\mathcal{W}$ of $\mathfrak{g}_{0}$ is the subgroup of $\mathrm{GL}\left(\mathfrak{H}^{*}\right)$ generated by permutations of indices and sign changes of the spin basis.

2. For even $d$, the Weyl group $\mathcal{W}$ of $\mathfrak{g}_{0}$ is the subgroup of $\mathrm{GL}\left(\mathfrak{H}^{*}\right)$ generated by permutations of indices and even numbers of sign changes of the spin basis.

Proof. Because of lemma 1.6.5, the scalar product on $\mathfrak{S}^{*}$ is $\left(\gamma_{i}, \gamma_{j}\right)=c \cdot \delta_{i, j}$ with a constant $c \in \mathbb{Z}$ that depends on $d$. Using this and formula (2.25) we calculate the value of the reflections $\sigma_{\alpha_{i}}$ that generate the Weyl group on the basis vectors $\gamma_{i}$ of $\mathfrak{H}^{*}$ (only the results are given).

1. For odd $d$ :

$$
\begin{aligned}
\sigma_{\alpha_{1}}\left(\gamma_{1}\right) & =-\gamma_{1}, \\
\sigma_{\alpha_{i}}\left(\gamma_{i-1}\right) & =\gamma_{i}, \\
\sigma_{\alpha_{i}}\left(\gamma_{i}\right) & =\gamma_{i-1}, \\
\sigma_{\alpha_{i}}\left(\gamma_{j}\right) & =\gamma_{j} \text { for }|i-j| \geq 2 .
\end{aligned}
$$

This means that $\sigma_{\alpha_{1}}$ corresponds to the linear operator replacing $\pm_{1}$ by $\mp_{1}$ ("changing the first sign"), and the $\sigma_{\alpha_{i}}$ with $i>1$ to the one replacing $\pm_{i}$ by $\pm_{i-1}$ and vice versa. Since such transpositions generate the symmetric group, all other permutations can be written as compositions of several $\sigma_{\alpha_{i}}, i>1$. Changing other signs can be written as a composition of permutations and changing the first sign.

2. For even $d$ :

$$
\begin{aligned}
\sigma_{\alpha_{0}}\left(\gamma_{0}\right) & =-\gamma_{1}, \\
\sigma_{\alpha_{0}}\left(\gamma_{1}\right) & =-\gamma_{0}, \\
\sigma_{\alpha_{i}}\left(\gamma_{i-1}\right) & =\gamma_{i}, \\
\sigma_{\alpha_{i}}\left(\gamma_{i}\right) & =\gamma_{i-1}, \\
\sigma_{\alpha_{i}}\left(\gamma_{j}\right) & =\gamma_{j} \text { for }|i-j| \geq 2 .
\end{aligned}
$$

The rest of the argument is analogous to the odd case, with the exception that $\sigma_{\alpha_{0}} \sigma_{\alpha_{1}}$ changes of the 0th and 1st signs simultaneously. 
These symmetries were known to Pohlmeyer (at least for the Poisson algebra of invariant charges $\mathfrak{h}$ constructed from $\mathfrak{g}$ in 3.2.3) and his collaborators (for instance, cf. [Poh94, p. 632]) and can be seen as arising from sign changes in spacetime coordinates .

\subsection{Weight space decomposition}

\subsubsection{Overview of the general theory}

Since no Cartan subalgebra of $\mathfrak{g}$ is known and the sitation is more complicated in the infinitedimensional case, we cannot use the same approach we took to the structure of $\mathfrak{g}_{0}$ (in subsection 2.2.4) to the entire Lie algebra g. However, by lemma 2.2.2, $\mathfrak{g}$, and indeed each individual stratum $\mathfrak{g}_{l}$, is a module of the subalgebra $\mathfrak{g}_{0}$, and so we can make use of the well-developed theory of representations of semisimple Lie algebras, in particular the weight space decomposition, to better understand the structure of the Pohlmeyer-Rehren Lie algebra g. Before we go into the specifics of the Pohlmeyer-Rehren Lie algebra, we begin with an overview of some of the needed basic definitions and facts, mainly adapted from [Hei90, p. 235ff.].

Definition 2.3.1 (weight, highest weight, multiplicity). Let $G$ be a semisimple finite-dimensional complex Lie algebra, $H$ a Cartan subalgebra of $G$ and $\left\{\alpha_{1}, \ldots, \alpha_{n}\right\}$ a base of $G$. Let further be $V$ be a finite-dimensional G-module via the representation $\rho: G \rightarrow \mathfrak{g l}(V)$.

1. A linear form $\lambda \in H^{*}$ is called a weight of $V$ (with respect to $H$ ) if

$$
V^{\lambda}:=\{v \in V \mid \rho(h)(v)=\lambda(h) v \forall h \in H\} \neq\{0\} .
$$

We then call $V^{\lambda}$ the weight space of $\lambda$. The set of all weights of a module is designated by $\Gamma$.

2. A weight $\lambda \in \Gamma$ is called a highest weight if

$$
\lambda+\alpha \notin \Gamma \text { for all } \alpha \in R^{+} .
$$

Then, every $v \in V^{\lambda} \backslash\{0\}$ is called a highest weight vector $\}^{3}$

3. Let $\mu \in H^{*}$. Define the multiplicity of $\mu$ (with respect to the G-module $V$ ) as

$$
n_{V}(\mu):=\left\{\begin{array}{ll}
\operatorname{dim} V^{\mu} & \text { if } \mu \in \Gamma \\
0 & \text { else }
\end{array} .\right.
$$

If the $G$-module meant is unambiguous, the index $V$ can be suppressed.

Not only is the defining equation (2.70) of weights and weight spaces similar to the defining equation (2.21) of roots and root spaces; in fact, any Lie algebra is a module of itself via the adjoint representation. A quick comparison of the definitions shows that the weights

\footnotetext{
${ }^{3}$ By replacing condition 2.71 by

$$
\lambda+\alpha \notin \Gamma \text { for all } \alpha \in R^{-},
$$

one analogously obtains a definition of the lowest weight and lowest weight vectors. With this, the theory works analogously when interchanging positive and negative roots in all other places as well.
} 
of this module are exactly the roots, with the exception that 0 is allowed as a weight, but not as a root. Because of this, the weight space $G^{0}$ is not a root space - instead it is the Cartan subalgebra $H$. In this sense, the theory of weights is a natural extension of the theory of roots. Let us now recount some details of the theory of weights that highlight their importance.

Theorem 2.3.2. Under the preconditions of definition 2.3.1.

1. we have the weight space decomposition

$$
V=\bigoplus_{\lambda \in \Gamma} V^{\lambda}
$$

2. $\rho\left(x_{\alpha}\right)\left(V^{\lambda}\right) \subset V^{\lambda+\alpha}$ for all $\alpha \in R, \lambda \in H^{*}$.

3. a highest weight exists.

Proof. This is the lemma from [Hei90, p.236]

Theorem 2.3.3. Let now in addition to the preconditions of definition 2.3.1 be the G-module $V$ irreducible, let $v_{0}$ be a highest weight vector of weight $\sigma$, and let $y_{i}$ be as in remark 2.2.16. Then the following statements hold.

1. $V$ is spanned by the vectors

$$
\rho\left(y_{i_{1}}\right) \ldots \rho\left(y_{i_{k}}\right)\left(v_{0}\right), i_{v} \in I, k \in \mathbb{N}_{0} .
$$

2. Every weight of $\rho$ can be written as

$$
\sigma-\sum_{i=1}^{n} k_{i} \alpha_{i}
$$

with coefficients $k_{i} \in \mathbb{N}_{0}$ for all $i \in 1, \ldots, n$

3. $n(\sigma)=1$.

4. $\sigma$ is the only highest weight.

5. Let $V^{\prime}$ be another irreducible G-module with highest weight $\sigma^{\prime}$. Then $V$ and $V^{\prime}$ are equivalent if and only if $\sigma=\sigma^{\prime}$.

Proof. See [Hei90, p. 236f] or [Hum72, p.107ff].

Since weights can be thought of as a generalization of roots, it is not surprising that the Weyl group also controls the symmetries of the weights.

Theorem 2.3.4. Let $G$ be a semisimple finite-dimensional complex Lie algebra with Weyl group $W$ and let $V$ be a finite-dimensional G-module. Then the set of weights $\Gamma$ of $\rho$ is permuted by $\mathcal{W}$ and

$$
n_{V} \sigma(\mu)=n_{V}(\sigma \mu)
$$

for all $\sigma \in \mathcal{W}$ and all $\mu \in \Gamma$.

Proof. See [Hum72, Thm. 21.2] 
In the context of the Pohlmeyer-Rehren Lie algebra and the Poisson algebra of invariants constructed from it (cf. chapter 3), the parity operation of exchanging plusses and minusses in the index words is known. The above theorem, along with lemma 2.2.17, put this in a general context.

Remark 2.3.5. In light of theorem 2.3.35, we can write $\rho_{\sigma}$ for a representation and ${ }^{\sigma} V$ for its corresponding module with highest weight $\sigma$. This defines $\rho_{\sigma}$ and ${ }^{\sigma} V$ uniquely up to equivalence/isomorphism. We also write

$$
n_{\sigma}(\mu)
$$

for the multiplicity of weight $\mu$ in a module of highest weight $\sigma$.

A way to calculate the multiplicities of certain weights is given by FreUdentHAL's formula.

Theorem 2.3.6 (Freudenthal's formula). Let $G$ be a semisimple complex Lie algebra with Cartan subalgebra $H$ and set of roots $R$. Let further $V$ be an irreducible $G$-module of highest weight $\sigma$. For all weights $\mu \in \Gamma$, the equation

$$
((\sigma+\delta, \sigma+\delta)-(\mu+\delta, \mu+\delta)) n(\mu)=2 \sum_{\alpha>0} \sum_{i=1}^{\infty}(\mu+i \alpha, \alpha) \cdot n(\mu+i \alpha)
$$

holds with

$$
\delta:=\frac{1}{2} \sum_{\alpha \in R^{+}} \alpha
$$

Proof. See [Hum72, Section 22.3].

Remark 2.3.7. Freudenthal's formula allows to calculate the multiplicities of the weights of a module recursively, starting from the highest weight $\lambda$, for which (by theorem 2.3.3 3) we already know the multiplicity $n(\lambda)=1$. In particular ${ }^{4}$, for irreducible $\mathfrak{s}(3, \mathbb{C})$-modules and $\mathfrak{s o}(4, \mathbb{C})$-modules, we have

$$
n(\mu)= \begin{cases}1 & \text { if }-\lambda>\mu>\lambda \\ 0 & \text { else }\end{cases}
$$

using the partial order $>$ defined in equation (2.23).

We conclude this overview of some basic theory of weights with a very important and well known result of representation theory that will have consequences in the next section.

Theorem 2.3.8 (WEYL's theorem on complete reducibility). Let $G$ be a semisimple Lie algebra and let $V$ be a finite-dimensional $G$-module. Then $V$ is completely reducible, i.e. a finite direct sum of irreducible G-modules.

Proof. See [Hum72, Section 6.3].

\footnotetext{
${ }^{4}$ This can alternatively be proved without using the Freudenthal formula using the fact that the multiplicities are invariant under the Weyl group by theorem 2.3.4 and the fact that for $d \in\{3,4\}$, the elements $y_{i}$ and with then their adjoints commute. There also is a Mathematica application called LieART ([FK15] $)$ that is able to calculate multiplicities of all weights for all finite-dimensional irreducible modules of the classical Lie algebras.
} 


\subsubsection{Application to the Pohlmeyer-Rehren Lie algebra}

After the preceding outlined of the general theory of weights, it can be applied to the Pohlmeyer-Rehren Lie algebra. We begin with some notations suited to the case at hand.

Remark 2.3.9 (notation). Due to their definition 2.3.1 as linear functionals on the Cartan subalgebra $H$, weights can be described by their values on a basis $\left(h_{i}\right)_{i \in I_{d}}$ of $H$, and the defining equation of the weight space becomes

$$
\mathfrak{g}_{l}^{\lambda}:=\left\{x \in \mathfrak{g} \mid \operatorname{ad}_{h_{i}}(x)=\lambda\left(h_{i}\right) x \forall i \in I_{d}\right\} .
$$

In other words, the weight space $\mathfrak{g}_{l}^{\lambda}$ is the common eigenspace of the adjunction of the basis elements of $H$. Consequently, the weight tuple $\left(\mu_{i}\right)_{i \in I_{d}}$ of their eigenvalues

$$
\mu_{i}:=\lambda\left(h_{i}\right)
$$

can be used to describe the weight $\lambda$ and the weight space $\mathfrak{g}_{l}^{\lambda}$. Therefore, we also write $\mathfrak{g}_{l}^{\left(\mu_{i}\right)_{i \in I_{d}}}$ instead of $\mathrm{g}_{l}^{\lambda}$.

Due to the form the basis of the Cartan subalgeba of $\mathfrak{g}_{0}$ given in remark 2.2.16 and lemma 1.6.5. the values $\mu_{i}$ can be expressed as sums and differences of magnetic quantum numbers, specifically, if $d$ is even,

$$
\begin{aligned}
\mu_{0} & =m_{0}+m_{1}, \\
\mu_{i} & =-m_{i-1}+m_{i} \text { for } i \in I_{d} \backslash\{0\},
\end{aligned}
$$

and, if $d$ is odd,

$$
\begin{aligned}
\mu_{1} & =2 m_{1}, \\
\mu_{i} & =-m_{i-1}+m_{i} \text { for } i \in I_{d} \backslash\{1\} .
\end{aligned}
$$

Since the map between the tuples $\left(\mu_{i}\right)_{i \in I_{d}}$ and $\left(m_{i}\right)_{i \in I_{d}}$ is a bijection, we can also use the magnetic quantum numbers to describe weights, writing $\mathfrak{g}_{l}^{\left(m_{i}\right)_{i \in I_{d}}}$ in a slight abuse of notation. Consequently, the weight spaces are just the vector spaces of elements having a particular tuple of magnetic quantum numbers. As magnetic quantum numbers can be read off somewhat more easily than the corresponding weights (by their definition 1.6.3 just by counting the differences of corresponding plusses and minusses), we will use them in the next theorem to describe the weight spaces and give a formula for their dimensions.

We can calculate how adjoining the positive and negative roots given in remark 2.2.16 connect the weight spaces:

$$
\begin{aligned}
\operatorname{ad}_{x_{j}} \mathfrak{g}_{l}^{\left(\mu_{i}\right)_{i \in I_{d}}} \subset \mathfrak{g}_{l}^{\left(\mu_{i}+2 \delta_{i j}\right)_{i \in I_{d}}}, \\
\operatorname{ad}_{y_{j}} \mathfrak{g}_{l}^{\left(\mu_{i}\right)_{i \in I_{d}}} \subset \mathfrak{g}_{l}^{\left(\mu_{i}-2 \delta_{i j}\right)_{i \in I_{d}}} .
\end{aligned}
$$

This notation for weights can be applied to highest weights of irreducible $\mathfrak{g}_{0}$-modules as well; define the highest weight tuple $\left(\sigma_{i}\right)_{i \in I_{d}}$ of the highest weight $\sigma$ of the irreducible $\mathfrak{g}_{0}$-module. Now, the highest weight $\sigma$ determines (again, using 2.3.35) the irreducible $\mathfrak{g}_{0}$-module up to isomorphism. 
Remark 2.3.10 (physicist's notation for $d=3$ ). In the special case $d=3$, the situation is particularly simple because $m:=m_{1}$ is the only magnetic quantum number and we have $\mu_{1}=2 m$, so it is convenient to use the magnetic quantum number directly instead of $\mu$ to refer to weights. The theory of irreducible representations of $\mathfrak{s l}_{2}$ (which is isomorphic to $\mathfrak{g}_{0}$ for $d=3$ ) is familiar in physics, where irreducible $\mathfrak{s l}_{2}$-modules (with highest weight of magnetic quantum number s) are called multiplets (of spin s) and the adjunctions $\mathrm{ad}_{x}$ and $\operatorname{ad}_{y}$ are called ladder operators. We will slightly generalize this terminology and call irreducible $\mathfrak{g}_{0}$-modules multiplets, regardless of the dimension $d$. Returning to $d=3$, in this notation, remark 2.3.7 means that any multiplet of spin $s$ has all integer weights $m$ such that $-s \leq m \leq s$, each with multiplicity 1. Expressed with magnetic quantum numbers, equations (2.85) become

$$
\begin{aligned}
& \operatorname{ad}_{x} \mathfrak{g}_{l}^{m} \subset \mathfrak{g}_{l}^{m+1}, \\
& \operatorname{ad}_{y} \mathfrak{g}_{l}^{m} \subset \mathfrak{g}_{l}^{m-1} .
\end{aligned}
$$

Write (cf. definition 2.3.13 $n_{s}(m)$ for the multiplicity of the weight corresponding to the magnetic quantum number $m$ in an irreducible $\mathfrak{g}_{0}$-module of highest weight $s$.

We can apply WeYL's theorem on complete reducibility to the finite-dimensional module $\mathfrak{g}_{l}$ of the semisimple Lie algebra $\mathfrak{g}_{0}$ and write $\mathfrak{g}_{l}$ as a finite direct sum of irreducible $\mathfrak{g}_{0}$-modules. These are determined (up to isomorphism) by their highest weight (denoted by $\sigma$ ), so we can pick any irreducible $\mathfrak{g}_{0}$-module with highest weight $\sigma$, call it ${ }^{\sigma} V$, and have an isomorphism of $\mathfrak{g}_{0}$-modules

$$
\mathfrak{g}_{l} \cong \bigoplus_{\left(\sigma_{i}\right)_{i \in I_{d}} \in \mathbb{N}_{0}^{I_{d}}} v_{l}(\sigma) \cdot{ }^{\sigma} V,
$$

using the notation

$$
v \cdot V:=\bigoplus_{i=1}^{v} V
$$

and allowing $v_{l}(\sigma)$ to be zero for $\mathfrak{g}_{0}$-modules that do not occur in the direct sum. An obvious question to ask is how many copies of each type of irreducible $\mathfrak{g}_{0}$-module there are for a given $l$, in other words what values the $v_{l}(\sigma)$ have. In this section, a theorem (2.3.13) answering this question using the weight space decomposition of $\mathfrak{g}_{l}$ will be given (we will revisit the motivation given above with a little more detail in the proof). We begin by treating the entire stratum $\mathfrak{g}_{l}$ as a single, reducible, $\mathfrak{g}_{0}$-module, considering the weight space decomposition (as in 2.3.2.

$$
\mathfrak{g}_{l}=\bigoplus_{\mu \in \mathbb{N}_{0}^{I_{d}}} \mathfrak{g}_{l}^{\mu}
$$

and applying the combinatorial theorem 1.3.6 on the number of Lyndon words with a specific letter content on the dimensions of the weight spaces. 
Theorem 2.3.11 (dimension of weight spaces). Using the notation $M:=\sum_{i \in I_{d}}\left|m_{i}\right|$ and $m_{i}^{ \pm}:=\frac{\left|m_{i}\right| \pm m_{i}}{2}$ as well as for any $x \in \mathbb{N}_{0}$,

$$
\operatorname{part}_{I_{d}}(x):=\left\{\pi \in \mathbb{N}_{0}^{I_{d}} \mid \sum_{i \in I_{d}} \pi_{i}=x\right\}
$$

the set of partitions of $x$ into $\# I_{d}=n$ nonnegative integer 5 . indexed by $I_{d}$, the dimension of the weight space $\mathfrak{g}_{l}^{m}$ can be expressed by the following formulas, using the following notation for multinomial coefficients

$$
\left(\begin{array}{c}
n \\
k_{i}, j_{i}
\end{array}\right)_{i \in I}:=\frac{n !}{\prod_{i \in I} k_{i} ! j_{i} !}, \quad\left(\begin{array}{c}
n \\
l, k_{i}, j_{i}
\end{array}\right)_{i \in I}:=\frac{n !}{l ! \cdot \prod_{i \in I} k_{i} ! j_{i} !}
$$

where I is a finite index set and $n, l, k_{i}, j_{i} \in \mathbb{N}_{0} \forall i \in I$.

1. For $d=2 n$, if $M+l=0 \bmod 2$, then

$$
\operatorname{dim} \mathfrak{g}_{l}^{m}=\frac{1}{l+2} \sum_{\pi \in \operatorname{part}_{I_{d}}\left(\frac{l+2-M}{2}\right)} \sum_{q\left|m_{i}, q\right| \pi_{i}} \mu(q)\left(\begin{array}{c}
(l+2) / q \\
\pi_{i} / q,\left(\left|m_{i}\right|+\pi_{i}\right) / q
\end{array}\right)_{i \in I_{d}} .
$$

If $M+l=1 \bmod 2$, then

$$
\operatorname{dim} \mathfrak{g}_{l}^{m}=0 .
$$

2. For $d=2 n+1$,

$$
\begin{aligned}
& \operatorname{dim} \mathfrak{g}_{l}^{m}=\frac{1}{l+2} \sum_{\left.r \in\left\{0, \ldots, L \frac{l+2-M}{2}\right\rfloor\right\}} \sum_{\pi \in \operatorname{part}_{I_{d}}(r)} \sum_{q|l+2, q| m_{i}, q \mid \pi_{i}} \mu(q) \\
& \cdot\left(\begin{array}{c}
(l+2) / q \\
(l+2-M-2 r) / q, \pi_{i} / q,\left(\left|m_{i}\right|+\pi_{i}\right) / q
\end{array}\right)_{i \in I_{d}} .
\end{aligned}
$$

Proof. Since by definition 1.6.3 the magnetic quantum numbers $m_{i}$ are the differences of the occurrences $t_{i}$ and $-{ }_{i}$, any word $w$ of length $l+2$ with $e(w)$ of magnetic quantum number tuple $m=\left(m_{i}\right)_{i \in I_{d}}$ must contain at least $m_{i}^{+}$plusses and $m_{i}^{-}$minuses for all indices $i \in I_{d}$. Since this fixes $M$ letters, the remaining $l+2-M$ spaces must be filled by a combination of letters that does not change the magnetic quantum numbers.

1. For $d=2 n$, if $l+2-M$ is odd, there is no way to satisfy the requirement on the $m_{i}$, proving equation (2.93). Otherwise, we can only fill in $(l+2-M) / 2$ pairs of corresponding plusses and minuses. In other words, we can choose any partition $\pi \in \operatorname{part}_{I_{d}}\left(\frac{l+2-M}{2}\right)$ and have the number of plusses/minuses relating to $i$ be $m_{i}^{ \pm}+\pi_{i}$. Theorem 1.3 .6 on the number of Lyndon words consisting of particular numbers of letters then yields

$$
\operatorname{dim} \mathfrak{g}_{l}^{m}=\frac{1}{l+2} \sum_{\pi \in \operatorname{part}_{I_{d}}\left(\frac{l+2-M}{2}\right)} \sum_{q \mid\left(m_{i}^{ \pm}+\pi_{i}\right)} \mu(q)\left(\begin{array}{c}
(l+2) / q \\
\left(m_{i}^{-}+\pi_{i}\right) / q,\left(m_{i}^{+}+\pi_{i}\right) / q
\end{array}\right)_{i \in I_{d}} .
$$

\footnotetext{
${ }^{5}$ For odd $d$, $\operatorname{part} I_{d}(x)=\operatorname{part}(x)_{n}$, the usual partition of $x$ into $n$ integers; for even $d$, the index is shifted by -1 . This is done solely to reflect the (unnecessary) special consideration of the letter 0 in the alphabet (cf. remark 1.4.6.
} 
Assertion 1 follows from two related facts. First, for all $i \in I_{d}$, we have $m_{i}^{+}=0$ or $m_{i}^{-}=0$, so the condition on $q$ in the last sum is equivalent to $q \mid m_{i}$ and $q \mid \pi_{i}$. Second, always one of the two corresponding values $m_{i}^{-}+\pi_{i}$ and $m_{i}^{+}+\pi_{i}$ occurring in the multinomial coefficients is $\left|m_{i}\right|+\pi_{i}$ while the other is $\pi_{i}$, and their order does not affect the multinomial coefficient.

2. Similarly, for $d=2 n+1$ we can begin filling the remaining $l+2-M$ positions with $r \in$ $\left\{0, \ldots,\left\lfloor\frac{l+2-M}{2}\right\rfloor\right\}$ pairs of plusses and minuses as above; then the remaining $l+2-M-2 r$ spaces must be filled with zeroes. Again, theorem 1.3.6 gives us the number of Lyndon words that match this construction:

$$
\begin{aligned}
\operatorname{dim} \mathfrak{g}_{l}^{m}=\frac{1}{l+2} & \sum_{\left.r \in\left\{0, \ldots, L \frac{l+2-M}{2}\right\rfloor\right\}} \sum_{\pi \in \operatorname{part}_{I_{d}}(r)} \sum_{q|l+2-M-2 r, q|\left(m_{i}^{ \pm}+\pi_{i}\right)} \mu(q) \\
& \cdot\left(\begin{array}{c}
(l+2-M-2 r) / q,\left(m_{i}^{-}+\pi_{i}\right) / q,\left(m_{i}^{+}+\pi_{i}\right) / q
\end{array}\right)_{i \in I_{d}} .
\end{aligned}
$$

The requirement $q \mid l+2-M-2 r$ can be shortened to $q \mid l+2$, since we also demand $q \mid m_{i}+\pi_{i}$, which implies $q \mid \sum\left(m_{i}^{ \pm}+\pi_{i}\right)=M+2 r$. Now, the same arguments from the end of the proof of 1 also prove 2 .

\section{Corollary 2.3.12.}

1. Let $\sigma$ be a permutation of $I_{d}$ and $\left(\alpha_{i}\right)_{i \in I_{d}} \in\{-1,1\}^{I_{d}}$. Define

$$
\tilde{m}_{i}:=\alpha_{i} m_{\sigma(i)}, \quad \tilde{m}:=\left(\tilde{m}_{i}\right)_{i \in I_{d}} .
$$

Then

$$
\operatorname{dim} \mathfrak{g}_{l}^{\tilde{m}}=\operatorname{dim} \mathfrak{g}_{l}^{m}
$$

2. If $M>l+2$, then

$$
\operatorname{dim} \mathfrak{g}_{l}^{m}=0
$$

3. If $\left|m_{i}\right|>l+1>0$ for any $i \in I_{d}$, then

$$
\operatorname{dim} \mathfrak{g}_{l}^{m}=0 .
$$

Proof. 1. The dimension formulas only use the absolute values of the $m_{i}$; the multinomial coefficients are invariant under permutation of their lower arguments.

2. In this case, the sets $\operatorname{part}_{I_{d}}\left(\frac{l+2-M}{2}\right)$ and $\operatorname{part}_{I_{d}}(r)$ are empty.

3. If $\left|m_{j}\right|>l+2$ for any $j \in I_{d}$, we are in case 2 . If $\left|m_{j}\right|=l+2$, then $\pi_{i}=0$ for all $i \in I_{d}$ (in the odd-dimensional case also $r=0$ ) and all multinomial coefficients occurring are equal to 1 . We are left with the sum

$$
\operatorname{dim} \mathfrak{g}_{l}^{m}=\frac{1}{l+2} \sum_{q \mid m_{j}} \mu(q) .
$$


The sum on the right hand side is zero due since $\left|m_{j}\right|>1$ and the identity

$$
\sum_{q \mid p} \mu(q)=\delta_{p 1}
$$

for the Möbius function with the Kronecker delta $\delta$ holds.

The results of the somewhat unwieldy formulas of theorem 2.3.11 for low degree and weights, already using the weight tuple notation, are tabulated on page 58 and 59 for $d=3$ and $d=4$ respectively.

We can now tackle the question how many multiplets of each highest weight there are in each stratum. For $d=4$, it is a bit more natural to use the weight tuples instead of the magnetic quantum numbers there because they reflect the action of $\mathfrak{g}_{0}$ in a somewhat more straightforward way (by equations (2.85) and (2.86)).

Theorem 2.3.13 (numbers of multiplets per stratum and highest weight).

1. For all $d, l \in \mathbb{N}_{0}$ with $d \geq 3$, the stratum $\mathfrak{g}_{l}$ can be decomposed into multiplets (irreducible $\mathfrak{g}_{0}$-modules):

$$
\mathfrak{g}_{l}=\bigoplus_{j \in J} j \mathfrak{g}_{l} \cong \bigoplus_{s \in \mathbb{N}_{0}^{I_{d}}} v_{l}(s) \cdot{ }^{s} V
$$

with a finite index set $J$.

2. For all $d, l \in \mathbb{N}_{0}$ with $d \geq 3$,

$$
\operatorname{dim} \mathfrak{g}_{l}^{m}=\sum_{s \in \mathbb{N}_{0}^{I_{d}}} v_{l}(s) n_{s}(m)
$$

3. For $d=3$ and for all $l \in \mathbb{N}_{0}$, no multiplet of highest weight $s>l+1$ occurs in $\mathfrak{g}_{l}$;

$$
v_{l}(s)=0 \text { for all } s>l+1 \text {. }
$$

4. For $d=3$, the number of multiplets of highest weight $s$ in $\mathfrak{g}_{l}$ is

$$
\begin{aligned}
v_{l}(s) & =\operatorname{dim} \mathfrak{g}_{l}^{s}-\sum_{k=s+1}^{l+1} v_{l}(k) \\
& =\operatorname{dim} \mathfrak{g}_{l}^{s}-\operatorname{dim} \mathfrak{g}_{l}^{s+1} \\
& =\frac{1}{l+2} \sum_{k=s}^{s+1}(-1)^{k-s} \sum_{i=k}^{\left\lfloor\frac{k+l+2}{2}\right\rfloor} \sum_{q|i, q| k, q \mid l+2} \mu(q)\left(\begin{array}{c}
l+2 / q \\
(l+2+k-2 i) / q,(i-k) / q, i / q
\end{array}\right)
\end{aligned}
$$

for all $l \in \mathbb{N}_{0}$.

5. In particular, for $d=3$ and for all $l \in \mathbb{N}_{0}$,

$$
v_{l}(l+1)=1 .
$$


6. For $d=4$, if $\sigma_{i}>l+2$ or $\sigma_{i}+l=1 \bmod 2$ for any $i \in\{0,1\}$, then

$$
v_{l}\left(\sigma_{0}, \sigma_{1}\right)=0 .
$$

7. For $d=4$, the number of multiplets of highest weight $\left(\sigma_{0}, \sigma_{1}\right)$ in $\mathfrak{g}_{l}$ is

$$
v_{l}\left(\sigma_{0}, \sigma_{1}\right)=\operatorname{dim} \mathfrak{g}_{l}^{\left(\sigma_{0}, \sigma_{1}\right)}-\operatorname{dim} \mathfrak{g}_{l}^{\left(\sigma_{0}, \sigma_{1}+2\right)}-\operatorname{dim} \mathfrak{g}_{l}^{\left(\sigma_{0}+2, \sigma_{1}\right)}+\operatorname{dim} \mathfrak{g}_{l}^{\left(\sigma_{0}+2, \sigma_{1}+2\right)} .
$$

Proof. 1. We return to the motivating considerations given before theorem 2.3.11 in greater detail. Recall that the individual strata $g_{l}$ are finite-dimensional modules of the semisimple Lie algebra $\mathfrak{g}_{0}$ by lemma 2.2.2. Therefore, using WEYL's theorem on complete reducibility 2.3.8, $\mathfrak{g}_{l}$ can be decomposed into a finite direct sum of irreducible $\mathfrak{g}_{0}$-modules ${ }_{j} \mathfrak{g}_{l}$ with $j \in J$, where $J$ is a finite index set. By theorem 2.3.3, these ${ }_{j} \mathfrak{g}_{l}$ are only determined (up to isomorphism) by their highest weight (indicated by the spin tuples $s_{j}$ ), so we can write

$$
\mathfrak{g}_{l}=\bigoplus_{j \in J}{ }_{j} \mathfrak{g}_{l}=\bigoplus_{\sigma \in \mathbb{N}_{0}^{I_{d}}} \bigoplus_{j \in J \mid \sigma_{j}=\sigma} j_{j} \cong \bigoplus_{\sigma \in \mathbb{N}_{0}^{I_{d}}} v_{l}(\sigma) \cdot{ }^{\sigma} V
$$

with

$$
v_{l}(\sigma):=\#\left\{j \in J \mid \sigma_{j}=\sigma\right\} .
$$

2. Both $\mathfrak{g}_{l}$ and ${ }^{\sigma} V$ are $\mathfrak{g}_{0}$-modules. Apply a weight space decomposition to both sides of equation (2.109), obtaining

$$
\bigoplus_{\mu} \mathfrak{g}_{l}^{\mu}=\mathfrak{g}_{l} \cong \bigoplus_{\sigma \in \mathbb{N}_{0}^{I_{d}}} v_{l}(\sigma) \cdot{ }^{\sigma} V=\bigoplus_{\sigma \in \mathbb{N}_{0}^{I_{d}}} v_{l}(\sigma) \cdot \bigoplus_{\mu}{ }^{\sigma} V^{\mu}=\bigoplus_{\mu} \underset{\sigma \in \mathbb{N}_{0}^{I_{d}}}{\bigoplus} v_{l}(\sigma) \cdot{ }^{\sigma} V^{\mu}
$$

Now compare the parts of equal weight and use the fact that $\operatorname{dim}^{\sigma} V^{\mu}=: n_{\sigma}(\mu)$ (cf. remark 2.3.5 and definition 2.3.13).

3. Due to $2.3 .7($ recall that $\mu=2 m)$,

$$
n_{s}(m)=\left\{\begin{array}{ll}
1 & \text { if }-s \leq m=\leq s \\
0 & \text { else }
\end{array},\right.
$$

so if $s>l+1$,

$$
0 \stackrel{2.3 .1233}{=} \operatorname{dim} \mathfrak{g}_{l}^{s} \stackrel{2.103}{=} \sum_{s^{\prime}=0}^{\infty} v_{l}\left(s^{\prime}\right) n_{s^{\prime}}(s) \stackrel{2.112}{=} \sum_{s^{\prime}=s}^{\infty} v_{l}\left(s^{\prime}\right),
$$

and since the $v_{l}(s)$ are nonnegative, the assertion follows.

4. Similarly,

$$
\operatorname{dim} \mathfrak{g}_{l}^{m} \stackrel{2.103}{=} \sum_{s=0}^{\infty} v_{l}(s) n_{s}(m) \stackrel{2.112}{=} \sum_{s=m}^{\infty} v_{l}(s) \stackrel{3}{=} \sum_{s=m}^{l+1} v_{l}(s),
$$

from which the rest follows using equation (2.94) for the value of the dimensions occurring. 
5. Here, in equation (2.105), $\operatorname{dim} \mathfrak{g}_{l}^{l+1}=1$ (because $e(0+\ldots+)$ is the only Euler-Lyndon word in $\mathfrak{g}_{l}$ with magnetic quantum number $l+1$ ) and $\operatorname{dim} \mathfrak{g}_{l}^{l+2}=0$ because of 3 .

6. Analogous to 3, using corollary 2.3.12/2 and equation (2.93) respectively instead of statement 3

7. Analogous to 4 .

Because the formulas for the number of multiplets are a bit unwieldy to calculate quickly by hand, values for low $l$ and $d=3$ as well as $d=4$ are tabulated below and on page 60 for $d=3$ and $d=4$ respectively.

\begin{tabular}{|r|r|rrrrrrrrrrrr|}
\hline$l$ & $\operatorname{dim} \mathfrak{g}_{l}$ & $d_{l}^{0}$ & $d_{l}^{1}$ & $d_{l}^{2}$ & $d_{l}^{3}$ & $d_{l}^{4}$ & $d_{l}^{5}$ & $d_{l}^{6}$ & $d_{l}^{7}$ & $d_{l}^{8}$ & $d_{l}^{9}$ & $d_{l}^{10}$ & $d_{l}^{11}$ \\
\hline 0 & 3 & 1 & 1 & & & & & & & & & & \\
1 & 8 & 2 & 2 & 1 & & & & & & & & \\
2 & 18 & 4 & 4 & 2 & 1 & & & & & & & \\
3 & 48 & 10 & 9 & 6 & 3 & 1 & & & & & & \\
4 & 116 & 22 & 21 & 14 & 8 & 3 & 1 & & & & & \\
5 & 312 & 56 & 51 & 38 & 23 & 11 & 4 & 1 & & & & \\
6 & 810 & 136 & 127 & 96 & 63 & 32 & 14 & 4 & 1 & & & \\
7 & 2184 & 348 & 323 & 256 & 172 & 98 & 46 & 17 & 5 & 1 & & & \\
8 & 5880 & 890 & 835 & 672 & 474 & 282 & 145 & 60 & 21 & 5 & 1 & & \\
9 & 16104 & 2332 & 2188 & 1805 & 1305 & 822 & 447 & 207 & 80 & 25 & 6 & 1 & \\
10 & 44220 & 6136 & 5798 & 4846 & 3603 & 2352 & 1353 & 668 & 286 & 100 & 29 & 6 & 1 \\
\hline
\end{tabular}

Table 2.1: Dimensions $d_{l}^{m}:=\operatorname{dim} \mathfrak{g}_{l}^{m}$ of the weight space of the weight indicated by magnetic quantum number $m$ in stratum $\mathfrak{g}_{l}$ for low $l$ and $m$ for $d=3$.

\begin{tabular}{|r|r|rrrrrrrrrrrr|}
\hline$l$ & $\operatorname{dimg}_{l}$ & $v_{l}(0)$ & $v_{l}(1)$ & $v_{l}(2)$ & $v_{l}(3)$ & $v_{l}(4)$ & $v_{l}(5)$ & $v_{l}(6)$ & $v_{l}(7)$ & $v_{l}(8)$ & $v_{l}(9)$ & $v_{l}(10)$ & $v_{l}(11)$ \\
\hline 0 & 3 & 0 & 1 & & & & & & & & & & \\
1 & 8 & 0 & 1 & 1 & & & & & & & & & \\
2 & 18 & 0 & 2 & 1 & 1 & & & & & & & & \\
3 & 48 & 1 & 3 & 3 & 2 & 1 & & & & & & & \\
4 & 116 & 1 & 7 & 6 & 5 & 2 & 1 & & & & & & \\
5 & 312 & 5 & 13 & 15 & 12 & 7 & 3 & 1 & & & & & \\
6 & 810 & 9 & 31 & 33 & 31 & 18 & 10 & 3 & 1 & & & & \\
7 & 2184 & 25 & 67 & 84 & 74 & 52 & 29 & 12 & 4 & 1 & & & \\
8 & 5880 & 55 & 163 & 198 & 192 & 137 & 85 & 39 & 16 & 4 & 1 & & \\
9 & 16104 & 144 & 383 & 500 & 483 & 375 & 240 & 127 & 55 & 19 & 5 & 1 & \\
10 & 44220 & 338 & 952 & 1243 & 1251 & 999 & 685 & 382 & 186 & 71 & 23 & 5 & 1 \\
\hline
\end{tabular}

Table 2.2: Number $v_{l}(s)$ of multiplets of spin $s$ in the stratum $\mathfrak{g}_{l}$ for $d=3$. 


\begin{tabular}{|c|c|c|c|c|c|c|}
\hline $\operatorname{dim} \mathfrak{g}_{0}^{\left(\mu_{0}, \mu_{1}\right)}$ & $\mu_{0}=0$ & $\mu_{0}=2$ & & & & \\
\hline$\mu_{1}=0$ & 2 & 1 & & & & \\
\hline$\mu_{1}=2$ & 1 & & & & & \\
\hline $\operatorname{dim} \mathfrak{g}_{1}^{\left(\mu_{0}, \mu_{1}\right)}$ & $\mu_{0}=1$ & $\mu_{0}=3$ & & & & \\
\hline$\mu_{1}=1$ & 3 & 1 & & & & \\
\hline$\mu_{1}=3$ & 1 & & & & & \\
\hline $\operatorname{dim} \mathfrak{g}_{2}^{\left(\mu_{0}, \mu_{1}\right)}$ & $\mu_{0}=0$ & $\mu_{0}=2$ & $\mu_{0}=4$ & & & \\
\hline$\mu_{1}=0$ & 8 & 6 & 1 & & & \\
\hline$\mu_{1}=2$ & 6 & 4 & 1 & & & \\
\hline$\mu_{1}=4$ & 1 & 1 & & & & \\
\hline $\operatorname{dim} \mathfrak{g}_{3}^{\left(\mu_{0}, \mu_{1}\right)}$ & $\mu_{0}=1$ & $\mu_{0}=3$ & $\mu_{0}=5$ & & & \\
\hline$\mu_{1}=1$ & 20 & 10 & 2 & & & \\
\hline$\mu_{1}=3$ & 10 & 5 & 1 & & & \\
\hline$\mu_{1}=5$ & 2 & 1 & & & & \\
\hline$\overline{\operatorname{dim}} \mathfrak{g}_{4}^{\left(\mu_{0}, \mu_{1}\right)}$ & $\mu_{0}=0$ & $\mu_{0}=2$ & $\mu_{0}=4$ & $\mu_{0}=6$ & & \\
\hline$\mu_{1}=0$ & 66 & 50 & 20 & 3 & & \\
\hline$\mu_{1}=2$ & 50 & 36 & 15 & 2 & & \\
\hline$\mu_{1}=4$ & 20 & 15 & 6 & 1 & & \\
\hline$\mu_{1}=6$ & 3 & 2 & 1 & & & \\
\hline $\operatorname{dim} \mathfrak{g}_{5}^{\left(\mu_{0}, \mu_{1}\right)}$ & $\mu_{0}=1$ & $\mu_{0}=3$ & $\mu_{0}=5$ & $\mu_{0}=7$ & & \\
\hline$\mu_{1}=1$ & 175 & 105 & 35 & 5 & & \\
\hline$\mu_{1}=3$ & 105 & 63 & 21 & 3 & & \\
\hline$\mu_{1}=5$ & 35 & 21 & 7 & 1 & & \\
\hline$\mu_{1}=7$ & 5 & 3 & 1 & & & \\
\hline 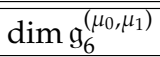 & $\mu_{0}=0$ & $\mu_{0}=2$ & $\mu_{0}=4$ & $\mu_{0}=6$ & $\mu_{0}=8$ & \\
\hline$\mu_{1}=0$ & 608 & 490 & 242 & 70 & 8 & \\
\hline$\mu_{1}=2$ & 490 & 392 & 196 & 56 & 7 & \\
\hline$\mu_{1}=4$ & 242 & 196 & 96 & 28 & 3 & \\
\hline$\mu_{1}=6$ & 70 & 56 & 28 & 8 & 1 & \\
\hline$\mu_{1}=8$ & 8 & 7 & 3 & 1 & & \\
\hline$\overline{\operatorname{dim}} \mathfrak{g}_{7}^{\left(\mu_{0}, \mu_{1}\right)}$ & $\mu_{0}=1$ & $\mu_{0}=3$ & $\mu_{0}=5$ & $\mu_{0}=7$ & $\mu_{0}=9$ & \\
\hline$\mu_{1}=1$ & 1764 & 1176 & 504 & 126 & 14 & \\
\hline$\mu_{1}=3$ & 1176 & 783 & 336 & 84 & 9 & \\
\hline$\mu_{1}=5$ & 504 & 336 & 144 & 36 & 4 & \\
\hline$\mu_{1}=7$ & 126 & 84 & 36 & 9 & 1 & \\
\hline$\mu_{1}=9$ & 14 & 9 & 4 & 1 & & \\
\hline $\operatorname{dim}_{g_{8}^{\left(\mu_{0}, \mu_{1}\right)}}$ & $\mu_{0}=0$ & $\mu_{0}=2$ & $\mu_{0}=4$ & $\mu_{0}=6$ & $\mu_{0}=8$ & $\mu_{0}=10$ \\
\hline$\mu_{1}=0$ & 6350 & 5292 & 3024 & 1134 & 252 & 25 \\
\hline$\mu_{1}=2$ & 5292 & 4400 & 2520 & 940 & 210 & 20 \\
\hline$\mu_{1}=4$ & 3024 & 2520 & 1440 & 540 & 120 & 12 \\
\hline$\mu_{1}=6$ & 1134 & 940 & 540 & 200 & 45 & 4 \\
\hline$\mu_{1}=8$ & 252 & 210 & 120 & 45 & 10 & 1 \\
\hline$\mu_{1}=10$ & 25 & 20 & 12 & 4 & 1 & \\
\hline$\overline{\operatorname{dim}} \mathfrak{g}_{9}^{\left(\mu_{0}, \mu_{1}\right)}$ & $\mu_{0}=1$ & $\mu_{0}=3$ & $\mu_{0}=5$ & $\overline{\mu_{0}=7}$ & $\mu_{0}=9$ & $\mu_{0}=11$ \\
\hline$\mu_{1}=1$ & 19404 & 13860 & 6930 & 2310 & 462 & 42 \\
\hline$\mu_{1}=3$ & 13860 & 9900 & 4950 & 1650 & 330 & 30 \\
\hline$\mu_{1}=5$ & 6930 & 4950 & 2475 & 825 & 165 & 15 \\
\hline$\mu_{1}=7$ & 2310 & 1650 & 825 & 275 & 55 & 5 \\
\hline$\mu_{1}=9$ & 462 & 330 & 165 & 55 & 11 & 1 \\
\hline$\mu_{1}=11$ & 42 & 30 & 15 & 5 & 1 & \\
\hline
\end{tabular}

Table 2.3: Dimensions $\operatorname{dim} \mathfrak{g}_{l}^{\left(\mu_{0}, \mu_{1}\right)}$ of the weight space of the weight $\left(\mu_{0}, \mu_{1}\right)$ in stratum $\mathfrak{g}_{l}$ for low $l$ and $\mu_{0}, \mu_{1}$ for $d=4$. 


\begin{tabular}{|c|c|c|c|c|c|c|}
\hline$v_{0}\left(\sigma_{0}, \sigma_{1}\right)$ & $\sigma_{0}=0$ & $\sigma_{0}=2$ & & & & \\
\hline$\sigma_{1}=0$ & 0 & 1 & & & & \\
\hline$\sigma_{1}=2$ & 1 & & & & & \\
\hline$v_{1}\left(\sigma_{0}, \sigma_{1}\right)$ & $\sigma_{0}=1$ & $\sigma_{0}=3$ & & & & \\
\hline$\sigma_{1}=1$ & 1 & 1 & & & & \\
\hline$\sigma_{1}=3$ & 1 & & & & & \\
\hline$v_{2}\left(\sigma_{0}, \sigma_{1}\right)$ & $\sigma_{0}=0$ & $\overline{\sigma_{0}}=2$ & $\overline{\sigma_{0}}=4$ & & & \\
\hline$\sigma_{1}=0$ & 0 & 2 & 0 & & & \\
\hline$\sigma_{1}=2$ & 2 & 2 & 1 & & & \\
\hline$\sigma_{1}=4$ & 0 & 1 & & & & \\
\hline$v_{3}\left(\sigma_{0}, \sigma_{1}\right)$ & $\sigma_{0}=1$ & $\sigma_{0}=3$ & $\sigma_{0}=5$ & & & \\
\hline$\sigma_{1}=1$ & 5 & 4 & 1 & & & \\
\hline$\sigma_{1}=3$ & 4 & 3 & 1 & & & \\
\hline$\sigma_{1}=5$ & 1 & 1 & & & & \\
\hline$v_{4}\left(\sigma_{0}, \sigma_{1}\right)$ & $\sigma_{0}=0$ & $\sigma_{0}=2$ & $\sigma_{0}=4$ & $\sigma_{0}=6$ & & \\
\hline$\sigma_{1}=0$ & 2 & 9 & 4 & 1 & & \\
\hline$\sigma_{1}=2$ & 9 & 12 & 8 & 1 & & \\
\hline$\sigma_{1}=4$ & 4 & 8 & 4 & 1 & & \\
\hline$\sigma_{1}=6$ & 1 & 1 & 1 & & & \\
\hline$v_{5}\left(\sigma_{0}, \sigma_{1}\right)$ & $\sigma_{0}=1$ & $\sigma_{0}=3$ & $\sigma_{0}=5$ & $\sigma_{0}=7$ & & \\
\hline$\sigma_{1}=1$ & 28 & 28 & 12 & 2 & & \\
\hline$\sigma_{1}=3$ & 28 & 28 & 12 & 2 & & \\
\hline$\sigma_{1}=5$ & 12 & 12 & 5 & 1 & & \\
\hline$\sigma_{1}=7$ & 2 & 2 & 1 & & & \\
\hline$v_{6}\left(\sigma_{0}, \sigma_{1}\right)$ & $\sigma_{0}=0$ & $\sigma_{0}=2$ & $\overline{\sigma_{0}}=4$ & $\overline{\sigma_{0}}=6$ & $\sigma_{0}=8$ & \\
\hline$\sigma_{1}=0$ & 20 & 52 & 32 & 13 & 1 & \\
\hline$\sigma_{1}=2$ & 52 & 96 & 72 & 24 & 4 & \\
\hline$\sigma_{1}=4$ & 32 & 72 & 48 & 18 & 2 & \\
\hline$\sigma_{1}=6$ & 13 & 24 & 18 & 6 & 1 & \\
\hline$\sigma_{1}=8$ & 1 & 4 & 2 & 1 & & \\
\hline$v_{7}\left(\sigma_{0}, \sigma_{1}\right)$ & $\overline{\sigma_{0}=1}$ & $\sigma_{0}=3$ & $\overline{\sigma_{0}}=5$ & $\overline{\sigma_{0}=7}$ & $\sigma_{0}=9$ & \\
\hline$\sigma_{1}=1$ & 195 & 225 & 126 & 37 & 5 & \\
\hline$\sigma_{1}=3$ & 225 & 255 & 144 & 43 & 5 & \\
\hline$\sigma_{1}=5$ & 126 & 144 & 81 & 24 & 3 & \\
\hline$\sigma_{1}=7$ & 37 & 43 & 24 & 7 & 1 & \\
\hline$\sigma_{1}=9$ & 5 & 5 & 3 & 1 & & \\
\hline$v_{8}\left(\sigma_{0}, \sigma_{1}\right)$ & $\overline{\bar{c} \sigma_{0}=0}$ & $\overline{\bar{c} \sigma_{0}=2}$ & $\overline{\bar{c} \sigma_{0}=4}$ & $\overline{\sigma_{0}=6}$ & $\overline{\bar{\sigma} \sigma_{0}=8}$ & $\overline{\sigma_{0}}=10$ \\
\hline$\sigma_{1}=0$ & 166 & 388 & 310 & 152 & 37 & 5 \\
\hline$\sigma_{1}=2$ & 388 & 800 & 680 & 310 & 82 & 8 \\
\hline$\sigma_{1}=4$ & 310 & 680 & 560 & 265 & 67 & 8 \\
\hline$\sigma_{1}=6$ & 152 & 310 & 265 & 120 & 32 & 3 \\
\hline$\sigma_{1}=8$ & 37 & 82 & 67 & 32 & 8 & 1 \\
\hline$\sigma_{1}=10$ & 5 & 8 & 8 & 3 & 1 & \\
\hline$\overline{v_{9}\left(\sigma_{0}, \sigma_{1}\right)}$ & $\overline{\bar{c} \sigma_{0}=1}$ & $\overline{\bar{c} \sigma_{0}=3}$ & $\overline{\bar{\sigma} \sigma_{0}=5}$ & $\overline{\bar{c} \sigma_{0}=7}$ & $\overline{\sigma_{0}=9}$ & $\overline{\sigma_{0}}=11$ \\
\hline$\sigma_{1}=1$ & 1584 & 1980 & 1320 & 528 & 120 & 12 \\
\hline$\sigma_{1}=3$ & 1980 & 2475 & 1650 & 660 & 150 & 15 \\
\hline$\sigma_{1}=5$ & 1320 & 1650 & 1100 & 440 & 100 & 10 \\
\hline$\sigma_{1}=7$ & 528 & 660 & 440 & 176 & 40 & 4 \\
\hline$\sigma_{1}=9$ & 120 & 150 & 100 & 40 & 9 & 1 \\
\hline$\sigma_{1}=11$ & 12 & 15 & 10 & 4 & 1 & \\
\hline
\end{tabular}

Table 2.4: Number $v_{l}\left(\sigma_{0}, \sigma_{1}\right)$ of multiplets of a given highest weight indicated by $\sigma_{0}, \sigma_{1}$ in the stratum $\mathfrak{g}_{l}$ for $d=4$. 


\subsubsection{Explicit weight space decompositions of $\mathfrak{g}_{0}$ and $\mathfrak{g}_{1}$ for $d=3,4$}

Remark 2.3.14. If $G$ is a semisimple finite-dimensional Lie algebra, the theorems featured in subsection 2.3.1 give rise to a method for decomposing a finite-dimensional $G$-module $V$ (via the representation $\rho: G \rightarrow \mathfrak{g l}(V))$ into irreducible $G$-modules:

1. First, decompose $V$ into weight spaces

$$
V=\bigoplus_{\lambda \in \Gamma} V^{\lambda}
$$

by finding the eigenvectors in the defining equation of weight spaces (2.70). This is always possible by theorem 2.3.2 1 .

2. For each weight $\lambda \in \Gamma$, we now find the subspace of the weight space $V^{\lambda}$ for which $\lambda$ is the highest weight. This can be done using definition 2.3.1/2 and 2.3.2 2 as

$$
\left.\bigcap_{\alpha \in R^{+}} \operatorname{ker} \rho\left(x_{\alpha}\right)\right|_{V^{\lambda}}
$$

Pick a basis of this vector space. Note that this is not necessarily unique.

3. Because of 2.3.33, each of the basis vectors now is the highest weight vector of an irreducible G-module, and due to 2.3.31, we can reconstruct the entirety of each irreducible $G$-module by iterative action of the negative roots.

Remark 2.3.15. For the Pohlmeyer-Rehren Lie algebra, step 1 is particularly easy because the weight spaces are spanned by the Euler-Lyndon words of particular magnetic quantum numbers. Mathematica code for step 2 can be found in example B.6.4.

Remark 2.3.16. The method given above is not the only way to explicitly decompose a stratum $\mathfrak{g}_{l}$ of the Pohlmeyer-Rehren Lie algebra into irreducible $\mathfrak{g}_{0}$-modules. For instance, it is possible to find the direct sum of all multiplets of a given highest weight as the eigenspace of the Casimir operator; the eigenvalue depends on the highest weight. This is implemented in Mathematica in example B.6.3.

Remark 2.3.17. Note that for $d=3,4$ and $l=0,1$, the selection of a basis in step 2 is unique up to scalars because (as can be deduced from theorem 2.3.13) in these cases, there is at most one irreducible $\mathfrak{g}_{0}$-module of any given highest weight. For $l \geq 2$, this is not the case.

For $l=0$, a canonical basis consisting of the Chevalley generators of $\mathfrak{g}_{0}$ (as a subalgebra) was already described for the root space decomposition in remark 2.2.16. Since they will later feature as conjectured generators of $\mathfrak{g}_{\geq 1}$ - in any case, they generate a significant subalgebra - we take this opportunity to also define basis vectors ${ }^{s} v^{m}$ of the weight spaces ${ }^{s} \mathfrak{g}_{1}^{m}$ for $d=3$ and basis vectors ${ }^{\sigma_{0}, \sigma_{1}} v^{\mu_{0}, \mu_{1}}$ of the weight spaces ${ }^{\sigma_{0}, \sigma_{1}} \mathfrak{g}_{1}^{\mu_{0}, \mu_{1}}$ for $d=4$, and plot them in the diagrams below, arranged by their magnetic quantum numbers.

These basis vectors were calculated using the method given above (this is simplified by the fact that for $d=3$, there is only one negative root, and for $d=4, y_{0}$ and $y_{1}$ commute, so the right hand side of equation (2.74) only depends on the multiplicities of $\mathrm{ad}_{y_{0}}$ and $\mathrm{ad}_{y_{1}}$, not their order).

A point in the diagram represents a weight space in an irreducible $\mathfrak{g}_{0}$-module, a line between two weight spaces means that one can be obtained from the other by adjoining 
an appropriate $x_{i}$ or $y_{i}$, so the connected components of the graphs in the diagrams can be understood as the irreducible $\mathfrak{g}_{0}$-modules. Highest weight vectors of each irreducible $\mathfrak{g}_{0^{-}}$ module are circled. Since we are only considering $\mathfrak{g}_{0}$ as a $\mathfrak{g}_{0}$-module instead of a subalgebra, the $x, y, h$ given below do not satisfy the canonical commutation relations of $\mathfrak{s l}_{2}$ (a basis that does is given in remark 2.2.16, but are scaled to allow for easy computations.

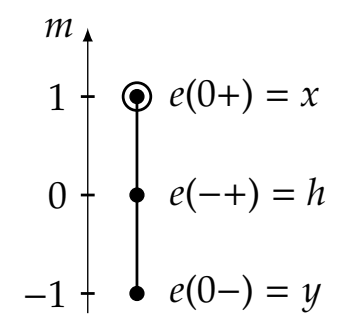

Figure 2.1: The subalgebra $\mathfrak{g}_{0}={ }^{1} \mathfrak{g}_{0}$ as the irreducible $\mathfrak{g}_{0}$-module via adjunction and its root spaces for $d=3$ as explained in remark 2.3.17.
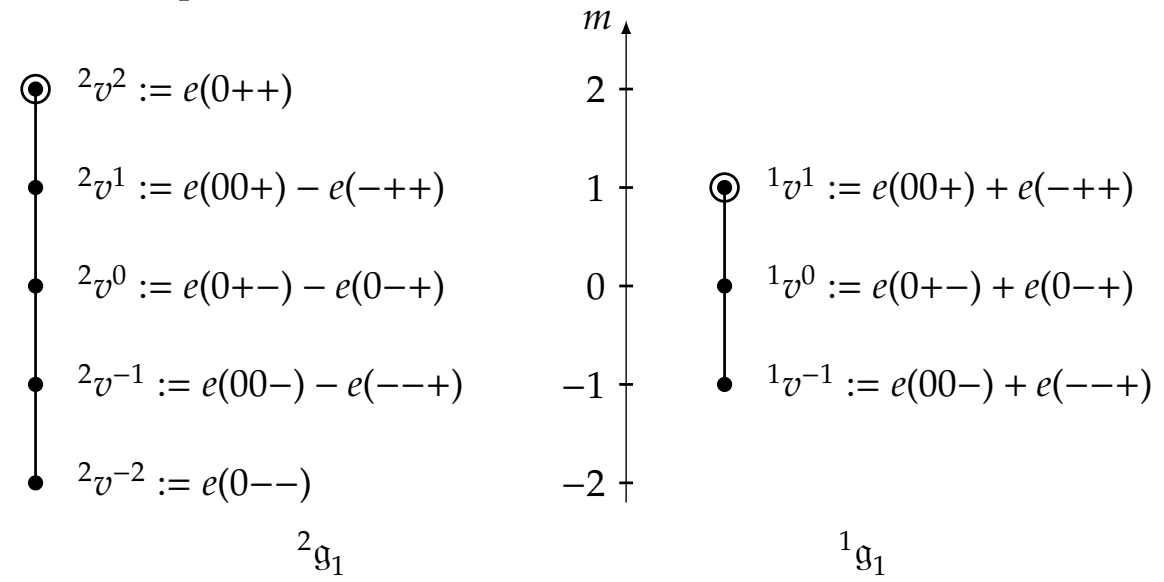

Figure 2.2: The two irreducible $\mathfrak{g}_{0}$-modules in the direct sum ${ }^{2} \mathfrak{g}_{1} \oplus{ }^{1} \mathfrak{g}_{1}=\mathfrak{g}_{1}$ and their weight spaces ${ }^{s} \mathfrak{g}_{1}^{m}$ (indicated by one of their respective basis vectors ${ }^{s} v^{m}$ each) for $d=3$ as explained in remark 2.3.17.
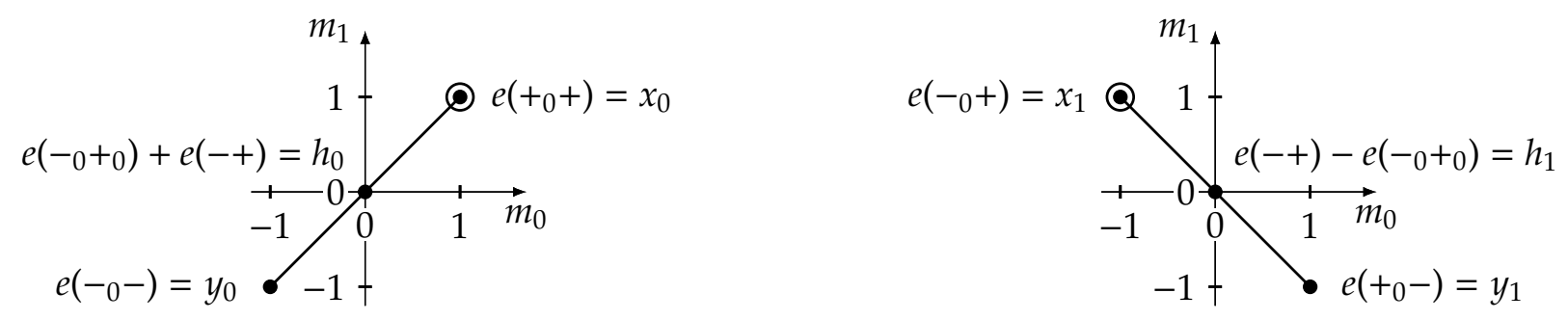

Figure 2.3: The subalgebra $\mathfrak{g}_{0}={ }^{2,0} \mathfrak{g}_{0} \oplus^{0,2} \mathfrak{g}_{0}$ as the $\mathfrak{g}_{0}$-module via adjunction and the root spaces of its irreducible components for $d=4$ as explained in remark 2.3.17. 

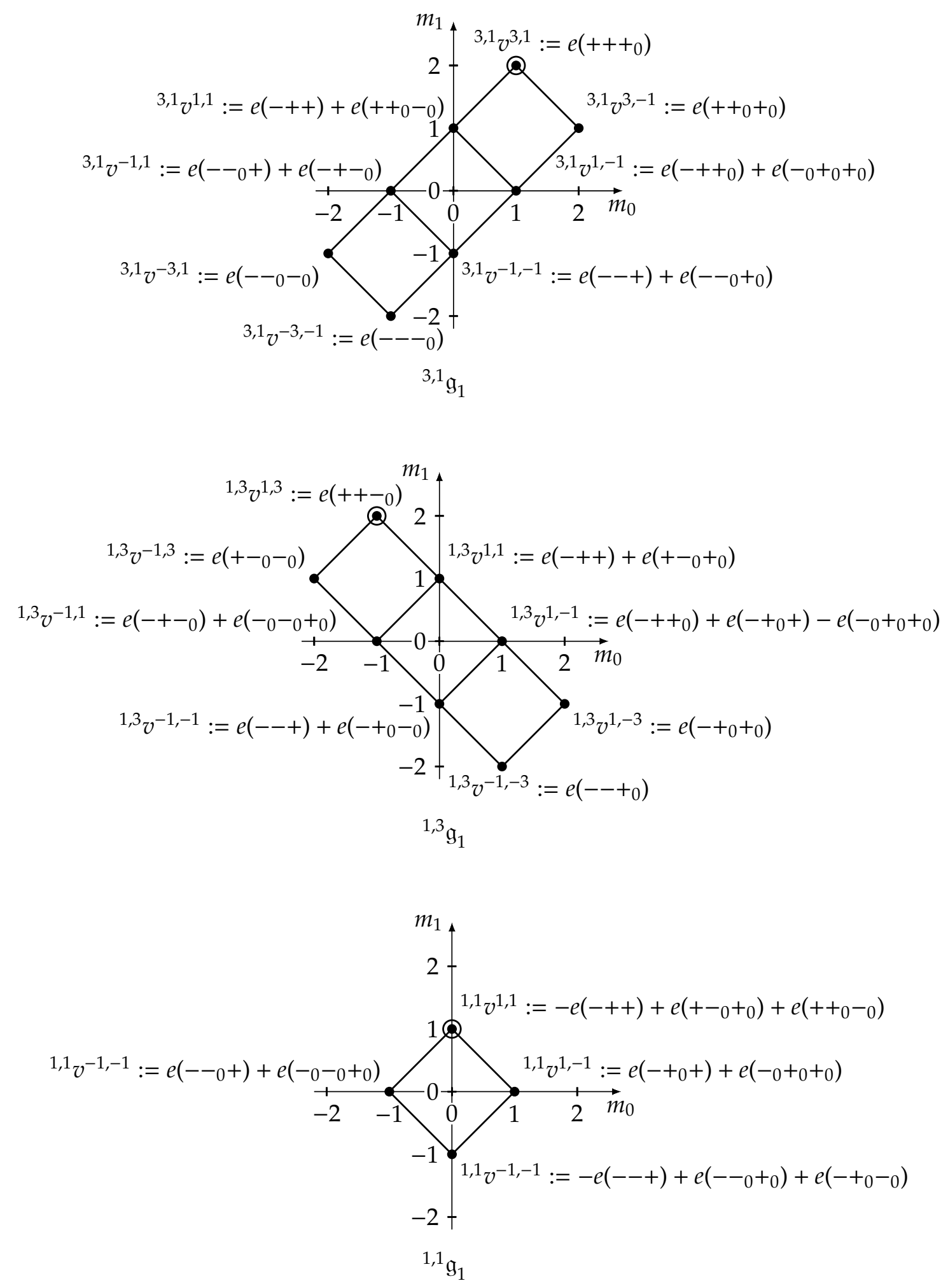

Figure 2.4: The three irreducible $\mathfrak{g}_{0}$-modules in the direct sum ${ }^{3,1} \mathfrak{g}_{1} \oplus^{1,3} \mathfrak{g}_{1} \oplus^{1,1} \mathfrak{g}_{1}=\mathfrak{g}_{1}$ and their weight spaces ${ }^{\sigma_{0}, \sigma_{1}} \mathrm{~g}_{1}^{\mu_{0}, \mu_{1}}$ (indicated by one of their respective basis vectors ${ }^{\sigma_{0}, \sigma_{1}} v^{\mu_{0}, \mu_{1}}$ each) for $d=4$ as explained in remark 2.3.17. 
In 1988, Pohlmeyer and Rehren gave [PR88] a maximal abelian subalgebra of the algebra of invariant charges $\mathfrak{h}$, the construction of which from the Pohlmeyer-Rehren Lie algebra $\mathfrak{g}$ will be outlined in chapter 3. Unfortunately, no obvious sign of a similar structure in $\mathfrak{g}$ presented itself. Nevertheless, the question of large abelian subalgebras of $\mathfrak{g}$ is interesting.

Can, in particular, the Cartan subalgebra of the stratum $\mathfrak{g}_{0}$ (that acts upon $\mathfrak{g}$ ) be expanded to an abelian subalgebra of the entire Lie algebra $\mathfrak{g}$ ? We begin to explore this question for our toy model $d=3$ where the Cartan subalgebra is spanned by the single element $h$. Other abelian subalgebras of $\mathfrak{g}$ of dimension greater than 1 exist.

Proposition 2.3.18 (Abelian subalgebras of $\mathfrak{g}$ for $d=3$ ). $\quad$ 1. $\mathfrak{g}^{0}$ lies in the centralizer (cf. defining equation (4.27) of $h$.

2. But $\mathrm{g}^{0}$ is not an abelian subalgebra of $\mathfrak{g}$.

3. It is not even possible to construct an infinite-dimensional abelian subalgebra of $\mathfrak{g}$ by picking a single element $x_{l}$ out of each $\mathfrak{g}_{l}^{0}$ such that all $x_{l}, l \in \mathbb{N}_{0} \cup\{-1\}$ commute.

4. The elements

$$
e(0 \pm \ldots \pm)
$$

(only plusses or only minusses) span infinite-dimensional abelian subalgebras of $\mathfrak{g}$.

Proof. 1. This is a direct consequence of lemma 1.6.5.

2. The Lie bracket of the two basis vectors ${ }^{1} v^{0},{ }^{2} v^{0}$ of $\mathfrak{g}_{1}^{0}$ given in remark 2.3 .17 is

$$
\left[{ }^{1} v^{0},{ }^{2} v^{0}\right]=2 \alpha(e(00-+)+e(00+-)+e(0-0+)+2 e(--++)) \neq 0 .
$$

3. Consider $l_{1}=1$ and $l_{2}=2$. Bases for $\mathfrak{g}_{1}^{0}$ and $\mathfrak{g}_{2}^{0}$ are given in 2.3.17 and B.6.3 respectively. Pick general elements of $x_{1}$ and $x_{2}$, and calculate their Lie bracket. This Lie bracket vanishes if and only if all coefficients of the Euler-Lyndon-elements vanish. This leads to a system of equations for the coefficients that is only solved by $x_{1}=0$ or $x_{2}=0$.

4. Obviously, these elements span an infinite-dimensional subvectorspace of $\mathfrak{g}$. It is in fact an abelian subalgebra because of corollary 2.3.12 2.

While statements 1., 2. and 4. also hold (or can be extended) for arbitrary $d$ and similar calculations to the proof of 3 . can be performed, we leave the context because these results are negative or, in the case of 4., do not have an easy application.

For instance, the abelian subalgebras considered in 4 . are not Cartan subalgebras because they are not self-normalizing.

This follows from the decomposition of $\mathfrak{g}_{l}$ into irreducible $\mathfrak{g}_{0}$-modules of which the elements considered above are highest (or lowest) weight vectors - in fact, the highest (or lowest) weight occurring in the entire stratum $\mathfrak{g}_{l}$. Therefore, adjunction of either $x$ or $y$ does not vanish. 


\subsection{Lie brackets of multiplets from the viewpoint of tensor and exterior products}

So far we have decomposed the individual strata of the Pohlmeyer-Rehren Lie algebra into irreducible $\mathfrak{g}_{0}$-modules and further decomposed those into weight spaces. While this took into account the action of $\mathfrak{g}_{0}$, it fails to illuminate the structure of the Lie brackets of all other elements. The following basic consideration of tensor products of irreducible modules of subalgebras of a given Lie algebra provides a starting point for this. We begin with a lemma highlighting the structural similarities between the tensor product and the Lie bracket.

Lemma 2.4.1. Let $G$ be a Lie algebra with subalgebra $H$. Let $V, V^{\prime} \subset G$ be H-modules via the adjunction. Then:

1. $\left[V, V^{\prime}\right]$ is a H-module (using the adjunction).

2. The tensor product $V \otimes V^{\prime}$ is a $H$-module using the action

$$
h .\left(v \otimes v^{\prime}\right)=h . v \otimes v^{\prime}+v \otimes h . v^{\prime}
$$

for all $h \in H, v \in V$ and $v^{\prime} \in V^{\prime}$.

3. The exterior product $V \wedge V$ is a H-module using the action

$$
h .\left(v \wedge v^{\prime}\right)=h . v \wedge v^{\prime}+v \wedge h \cdot v^{\prime}
$$

for all $h \in H$ and $v, v^{\prime} \in V$.

4. Let further designate

$$
\begin{aligned}
\psi: V \times V^{\prime} & \rightarrow V \otimes V^{\prime}, \\
\left(v, v^{\prime}\right) & \mapsto v \otimes v^{\prime} .
\end{aligned}
$$

Then the map

$$
\begin{aligned}
\Psi: V \otimes V^{\prime} & \rightarrow\left[V, V^{\prime}\right], \\
v \otimes v^{\prime} & \mapsto\left[v, v^{\prime}\right]
\end{aligned}
$$

is the unique linear map such that the diagram

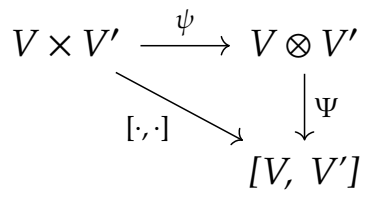

commutes. Furthermore, $\Psi$ is an epimorphism of $H$-modules. 
5. Let similarly designate

$$
\begin{aligned}
\varphi: V \times V & \rightarrow V \wedge V, \\
\left(v, v^{\prime}\right) & \mapsto v \wedge v^{\prime} .
\end{aligned}
$$

Then the map

$$
\begin{aligned}
\Phi: V \wedge V & \rightarrow[V, V] \\
v & \wedge v^{\prime} \mapsto\left[v, v^{\prime}\right]
\end{aligned}
$$

is the unique linear map such that the diagram

$$
V \times V \stackrel{\varphi}{\longrightarrow} V \wedge V
$$

commutes. $\Phi$ is an epimorphism of H-modules as well.

Proof. 1. This can be proved with a simple calculation using the facts that (due to the Jacobi identity), the adjunction is a derivation, equal terms with opposing signs can be eliminated, $V$ and $V^{\prime}$ are $H$-modules by assumption, and finally, again, that the adjunction is a derivation. Let $v \in V, v^{\prime} \in V^{\prime}$ and $h, h^{\prime} \in H$ and calculate

$$
\begin{aligned}
& h \cdot h^{\prime} \cdot\left[v, v^{\prime}\right]-h^{\prime} \cdot h \cdot\left[v, v^{\prime}\right] \\
= & h \cdot\left(\left[h^{\prime} \cdot v, v\right]+\left[v, h^{\prime} \cdot v^{\prime}\right]\right)-h^{\prime} .\left([h \cdot v, v]+\left[v, h \cdot v^{\prime}\right]\right) \\
= & {\left[h \cdot h^{\prime} \cdot v, v^{\prime}\right]+\left[h^{\prime} \cdot v, h \cdot v^{\prime}\right]+\left[h \cdot v, h^{\prime} \cdot v^{\prime}\right]+\left[v, h \cdot h^{\prime} \cdot v^{\prime}\right] } \\
& -\left[h^{\prime} \cdot h \cdot v, v^{\prime}\right]-\left[h \cdot v, h^{\prime} \cdot v^{\prime}\right]-\left[h^{\prime} \cdot v, h \cdot v^{\prime}\right]-\left[v, h^{\prime} \cdot h \cdot v^{\prime}\right] \\
= & {\left[\left[h, h^{\prime}\right] \cdot v, v^{\prime}\right]+\left[v,\left[h, h^{\prime}\right] \cdot v^{\prime}\right] } \\
= & {\left[h, h^{\prime}\right] \cdot\left[v, v^{\prime}\right] . }
\end{aligned}
$$

2. A calculation very similar to the proof of 1

3. Again, a very similar calculation.

4. Any Lie bracket is a bilinear map; in this situation the universal property of the tensor product guarantees that there is exactly one linear map such that the diagram commutes. It is just a matter of notation to check that $\Psi$ satisfies this and (simply writing out the respective actions of $H$ ) is an epimorphism of $H$-modules. Since $v \otimes v^{\prime}$ is a preimage of $\left[v, v^{\prime}\right]$ for all $v \in V, v^{\prime} \in V^{\prime}$, the map $\Psi$ is surjective.

5. Analogous.

If $V$ and $V^{\prime}$ are finite-dimensional $H$-modules, then $V \otimes V^{\prime}$ is a finite-dimensional module as well, and so is its image $\Psi\left(V \otimes V^{\prime}\right)=\left[V, V^{\prime}\right]$. If furthermore $H$ is semisimple, then we can apply WEYL's theorem on complete reducibility (2.3.8) on both $V \otimes V^{\prime}$ and $\left[V, V^{\prime}\right]$ and decompose them into a direct sum of irreducible $H$-modules. For $V \otimes V^{\prime}$, this is known as 
the Clebsch-Gordan problem, and with the epimorphism of $H$-modules $\Psi$ (or, if $V=V^{\prime}$, the epimorphism $\Phi)$ we can transfer some of the information to $\left[V, V^{\prime}\right]$. For instance, we have the estimate of dimensions

$$
\operatorname{dim}\left[V, V^{\prime}\right]=\operatorname{dim} \Psi\left(V \otimes V^{\prime}\right) \leq \operatorname{dim} V \otimes V^{\prime}=\operatorname{dim} V \cdot \operatorname{dim} V^{\prime} .
$$

Note that the maps $\Psi$ and $\Phi$ are not necessarily injective, so we only have the inequality $\operatorname{dim}\left[V, V^{\prime}\right] \leq \operatorname{dim} V \cdot \operatorname{dim} V^{\prime}$. We only consider the simplest case of $d=3$ to illustrate the method and its limitations. In this case, the Clebsch-Gordan problem has an easy solution.

Theorem 2.4.2 (Clebsch-Gordan formulas for $\mathfrak{s l}_{2}$ ). Let ${ }^{s} V$ designate irreducible $\mathfrak{s l}_{2}$-modules of highest weight $s$. Let $s_{1}, s_{2} \in \mathbb{N}_{0}$ with $s_{1} \geq s_{2}$. Then there are isomorphisms of $\mathfrak{s l}_{2}$-modules

$$
{ }^{s_{1}} V \otimes{ }^{s_{2}} V \cong \bigoplus_{s=s_{1}-s_{2}}^{s_{1}+s_{2}}{ }^{s} V
$$

and

$$
{ }^{s} V \wedge{ }^{s} V \cong \bigoplus_{n=1}^{s}{ }^{2 n-1} V .
$$

Proof. This is covered in many introductory texts on representations of Lie algebras, for instance [Hal03, Theorem D.1, note different notations for the modules of a given highest weight.]

We can use the above theorem to consider the generation of $\mathfrak{g}$ from certain multiplets, simplifying notation by suppressing the unnamed isomorphisms from the theorem for the remainder of this section. In subsection 2.3.3 we had seen that $\mathfrak{g}_{1}={ }^{2} \mathfrak{g}_{1} \oplus{ }^{1} \mathfrak{g}_{1} \cong{ }^{2} V \oplus{ }^{1} V$. So we calculate (note that $\left[{ }^{2} \mathfrak{g}_{1},{ }^{1} \mathfrak{g}_{1}\right]=\left[{ }^{1} \mathfrak{g}_{1},{ }^{2} \mathfrak{g}_{1}\right]$ ) due to antisymmetry of the Lie bracket)

$$
\begin{aligned}
& {\left[\mathfrak{g}_{1}, \mathfrak{g}_{1}\right]} \\
& =\left[{ }^{2} \mathfrak{g}_{1} \oplus^{1} \mathfrak{g}_{1},{ }^{2} \mathfrak{g}_{1} \oplus^{1} \mathfrak{g}_{1}\right] \\
& =\left[{ }^{2} \mathfrak{g}_{1},{ }^{2} \mathfrak{g}_{1}\right]+\left[{ }^{2} \mathfrak{g}_{1},{ }^{1} \mathfrak{g}_{1}\right]+\left[{ }^{1} \mathfrak{g}_{1},{ }^{2} \mathfrak{g}_{1}\right]+\left[{ }^{1} \mathfrak{g}_{1},{ }^{1} \mathfrak{g}_{1}\right] \\
& \cong \Phi\left({ }^{2} V \wedge{ }^{2} V\right)+\Psi\left({ }^{2} V \otimes{ }^{1} V\right)+\Phi^{\prime}\left({ }^{1} V \wedge{ }^{1} V\right) \\
& \stackrel{2.4 .2}{\cong} \Phi\left({ }^{3} V \oplus^{1} V\right)+\Psi\left({ }^{3} V \oplus^{2} V \oplus^{1} V\right)+\Phi^{\prime}\left({ }^{1} V\right) \text {. }
\end{aligned}
$$

Comparing this to the decompositon of $\mathfrak{g}_{2}$ into multiplets, by theorem 2.3.13 (cf. table 2.2)

$$
\mathfrak{g}_{2} \cong{ }^{3} V \oplus{ }^{2} V \oplus 2 \cdot{ }^{1} V,
$$

it becomes obvious that not all of the morphisms of $\mathfrak{s l}_{2}$-modules $\Phi, \Phi^{\prime}$ and $\Psi$ are injective or that (some of) the sums are not direct $[6$ In other words, there are relations between the

\footnotetext{
${ }^{6}$ By actually computing the decomposition of the Lie brackets of the pairs of irreducible modules in equation 2.128, for instance $\left[{ }^{2} \mathrm{~g}_{2},{ }^{2} \mathfrak{g}_{2}\right] \cong{ }^{3} V \oplus^{1} V$ and comparing this to the results of the Clebsch-Gordan formulas (theorem 2.4.2, for instance ${ }^{2} V \wedge{ }^{2} V \cong{ }^{3} V \oplus^{1} V$, one can see that the morphisms $\Phi, \Phi^{\prime}$ and $\Psi$ are all injective in this case. But the morphisms from lemma 2.4.1 are not neccessarily injective: take for example the multiplets ${ }^{2} \mathrm{~g}_{1} \cong{ }^{2} V$ and ${ }^{3} \mathfrak{g}_{2} \cong{ }^{3} V$ (which are uniquely determied by their highest weight and degree due to theorem 2.3.13 3). The decomposition of their Lie bracket is $\left[{ }^{2} \mathfrak{g}_{1},{ }^{3} \mathfrak{g}_{2}\right] \cong{ }^{4} V \oplus{ }^{3} V \oplus{ }^{2} V \oplus{ }^{1} V$, while by Clebsch-Gordan formula [2.126, ${ }^{2} V \otimes{ }^{3} V \cong{ }^{5} V \oplus^{4} V \oplus^{3} V \oplus^{2} V \oplus^{1} V$. In this case, the difference exists because there can be no module of type ${ }^{5} V$ in stratum $l=5$ due to theorem 2.3 .135
} 
elements of $\left[\mathfrak{g}_{1}, \mathfrak{g}_{1}\right]$ that do not follow from the axioms of the Lie algebra. So certain Lie brackets of elements of $\mathfrak{g}_{1}$ can be expressed as linear combinations of others. An obvious task arises from this: find a basis of $\mathfrak{g}_{l}$ in terms of multiple brackets of $\mathfrak{g}_{1}$ that exposes as much structure as possible. We begin with an educated guess based on the observation that if the summands originating from the term $\left[{ }^{2} \mathfrak{g}_{1},{ }^{2} \mathfrak{g}_{1}\right]$ were missing, we would neatly be in line with equation 2.129, with injective $\Phi^{\prime}$ and $\Psi$ and direct sums.

Generalizing this to higher strata $\mathfrak{g}_{l}$, we can ask the question if it is possible to obtain the entirety of $\mathfrak{g}_{l}$ with injective morphisms and direct sums by iteratively adjoining $l-1$ copies of ${ }^{1} \mathfrak{g}_{1}$ to $\mathfrak{g}_{1}={ }^{2} \mathfrak{g}_{1} \oplus{ }^{1} \mathfrak{g}_{1}$. For $l=2$, we unsurprisingly obtain the same number and types of multiplets that make up the entirety of $\mathfrak{g}_{2}$, but our conjecture fails for $\mathfrak{g}_{3}$ (again, cf. table 2.2) because

$$
\begin{aligned}
& {\left[{ }^{1} \mathfrak{g}_{1}, \mathfrak{g}_{2}\right]} \\
& \stackrel{[2.129}{=}\left[{ }^{1} \mathfrak{g}_{1},{ }^{3} V\right]+\left[{ }^{1} \mathfrak{g}_{1},{ }^{2} V\right]+\left[{ }^{1} \mathfrak{g}_{1},{ }^{1} V\right]+\left[{ }^{1} \mathfrak{g}_{1},{ }^{1} V\right] \\
& \cong \Psi\left({ }^{1} V \otimes{ }^{3} V\right)+\Psi^{\prime}\left({ }^{1} V \otimes{ }^{2} V\right)+\Psi^{\prime \prime}\left({ }^{1} V \otimes{ }^{1} V\right)+\Psi^{\prime \prime \prime}\left({ }^{1} V \otimes{ }^{1} V\right) \\
& \stackrel{2.4 .2}{\cong} \Psi\left({ }^{4} V \oplus^{3} V \oplus^{2} V\right)+\Psi^{\prime}\left({ }^{3} V \oplus^{2} V \oplus^{1} V\right)+\Psi^{\prime \prime}\left({ }^{2} V \oplus^{1} V \oplus^{0} V\right)+\Psi^{\prime \prime \prime}\left({ }^{2} V \oplus^{1} V \oplus^{0} V\right) \text {, }
\end{aligned}
$$

but the decomposition of $\mathfrak{g}_{3}$ into multiplets is

$$
\mathfrak{g}_{3} \cong{ }^{4} V \oplus 2 \cdot{ }^{3} V \oplus 3 \cdot{ }^{2} V \oplus 3 \cdot{ }^{1} V \oplus{ }^{0} V,
$$

so again, there are relations. Additionally, we do not know a priori whether the morphisms of $\mathfrak{s l}_{2}$-modules $\Phi, \Psi, \Psi^{\prime}$ etc. are injective in the first place or if they cause relations themselves. A way to approach this problem is needed, and chapter 4 provides a part of a solution. 


\title{
Chapter 3
}

\section{Excursus: String Quantization and Leading Terms of Exceptional Elements of the Invariant Algebra}

\begin{abstract}
After some light has been shed on the structure of the Pohlmeyer-Rehren Lie algebra in the previous chapter, in this excursion, a brief account will be given of some of the theory developed with quantization of the algebra of invariant charges of the Nambu-Goto string in mind. This serves a dual purpose: to give a motivation why the Pohlmeyer-Rehren Lie algebra merits study, and to provide some context for a result (proposition 3.4.3) and a conjecture 3.4.2) that will be introduced at the end of this chapter. The proof of this result itself is very straightforward, but to understand the terminology and relevance some explanation is required.
\end{abstract}

\subsection{Pohlmeyer's approach}

Pohlmeyer's approach to string quantization, which motivates this thesis, goes back to the 1980s, but the reformulation using the more mainstream mathematical language of the Shuffle Hopf algebra and the Eulerian idempotent is a more recent development due to BAHNS and Meinecke. The following three sections serve the dual purpose to provide a brief overview of the physical theory motivating this thesis as well as a point of connection to previous works that use the original notions. They mostly summarize BAHNs' and MeInECKE's account [BM11] as well as Meinecke's Diplomarbeit (in German) [Mei09, Chapters 1 and 2] and the original papers [Poh82], [Poh85], [PR86] [PR88], to which the reader is referred for more detail.

In the setting of the closed bosonic string with the Nambu-Goto (i.e. proportional to the worldsheet's surface area) action outlined in the introduction (page 9), POHLMEYER proposed [Poh82] the following approach. Recall from the introduction that the map

$$
x: S^{1} \times \mathbb{R} \rightarrow \mathbb{R}^{d}
$$

(such that for all $\tau$ the map $S^{1} \rightarrow \mathbb{R}^{d}, \sigma \mapsto x(\sigma, \tau)$ is a spacelike closed curve) parametrizes the string's worldsheet. Using a Lax pair, a system of linear differential equations describing the string's dynamics is set up and solved. The expansion of a power series (in the free parameters 
occurring in the monodromy matrix) of the monodromy matrix of the differential equations leads to coefficients $\mathcal{R}_{x_{1} \ldots x_{n}}^{ \pm}$where the indices ${ }^{1} x_{1}, \ldots, x_{n} \in\{0, \ldots, d-1\}$ correspond to the dimensions of spacetime for $n \in \mathbb{N}_{+}$. These coefficients are tensors expressed by the path ordered integrals

$$
\mathcal{R}_{x_{1} \ldots x_{n}}^{ \pm}(\sigma, \tau):=\int_{\sigma \leq \sigma_{n} \leq \ldots \leq \sigma_{1} \leq \sigma+2 \pi} u_{x_{1}}^{ \pm}\left(\sigma_{1}, \tau\right) \cdot \ldots \cdot u_{x_{n}}^{ \pm}\left(\sigma_{n}, \tau\right) d \sigma_{1} \ldots d \sigma_{n}
$$

with fixed $\sigma, \tau$, where the expressions $u^{ \pm}:=\partial_{\sigma} x \pm \partial_{\tau} x$ are the so-called left and right movers ${ }^{2}$ that are known from ordinary string theory, and $u_{x_{i}}^{ \pm}$is their $x_{i}$-th component.

A consequence of definition (3.1) of the $\mathcal{R}^{ \pm}$as path-ordered integrals is that they obey the multiplication rule

$$
\mathcal{R}_{x_{1} \ldots x_{n}}^{ \pm} \cdot \mathcal{R}_{y_{1} \ldots y_{m}}^{ \pm}=\mathcal{R}_{\left[\begin{array}{l} 
\pm \\
x_{1} \ldots x_{n} \\
y_{1} \ldots y_{m}
\end{array}\right.}^{ \pm} \cdot
$$

Since the algebraic relations between the mathematical objects introduced in this subsection only depend on the tensors' indices which are shuffled under multiplication, BAHNS and MeinecKe interpreted [BM11] the tensors $\mathcal{R}^{ \pm}$as words of their indices, the elements of the shuffle Hopf algebra (definition 1.1.2), and reformulated the remaining constructions - that will now be briefly introduced in their original forms - in these terms.

\subsubsection{The Poisson algebra of invariant charges}

The tensors $\mathcal{R}^{ \pm}$depend ${ }^{3}$ on the parameters $\sigma$ and $\tau$, but their cyclic symmetrizations,

$$
\mathcal{Z}_{x_{1} \ldots x_{n}}^{ \pm}(\sigma, \tau):=\mathcal{R}_{x_{1} \ldots x_{n}}^{ \pm}(\sigma, \tau)+\mathcal{R}_{x_{n} x_{1} \ldots x_{n-1}}^{ \pm}(\sigma, \tau)+\ldots+\mathcal{R}_{x_{2} \ldots x_{n} x_{1}}^{ \pm}(\sigma, \tau)
$$

could be shown to be independent of $\sigma$ and (if $x$ actually parametrizes the worldsheet, so that we are in the on-shell case also) $\tau$. Therefore, the $\mathcal{Z}^{ \pm}$are called the invariant charges (also known as Pohlmeyer charges) of the Nambu-Goto string.

A Poisson bracket on the invariant charges can be defined as follows:

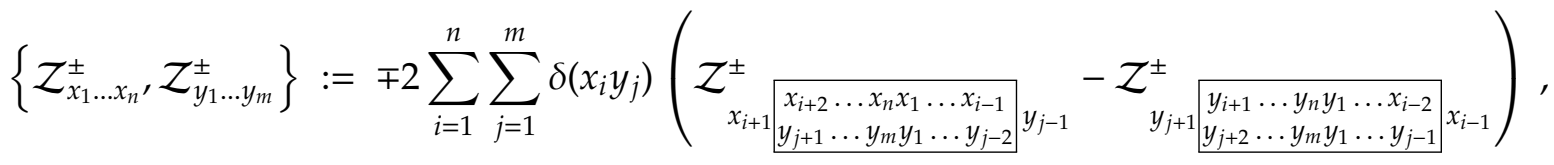

$$
\begin{aligned}
& \left\{\mathcal{Z}_{x_{1}}^{ \pm}, \mathcal{Z}_{y_{1} \ldots y_{n}}^{ \pm}\right\}:=0 ， \\
& \left\{\mathcal{Z}_{x_{1} \ldots x_{n}}^{ \pm}, \mathcal{Z}_{y_{1} \ldots y_{m}}^{\mp}\right\}:=0
\end{aligned}
$$

for $n, m \geq 2$. Since the Poisson brackets of $\mathcal{Z}^{ \pm}$and $\mathcal{Z}^{\mp}$ vanish and those of only one type differ only by a global sign, it has become customary to only focus on $\mathcal{Z}^{+}$and suppress the superscript + in the notation.

\footnotetext{
${ }^{1}$ Note that the indices $x_{i}$ are not components of the parametrization $x$. This somewhat confusing notation in this section was chosen for the sake of consistency with the rest of this thesis, where no risk of such a confusion exists.

${ }^{2}$ The left and right movers also have the property that their lightlikeness is equivalent to the constraints on the canonical coordinates on the string.

${ }^{3}$ By choosing an appropriate gauge, $\partial_{\tau} \mathcal{R}^{ \pm}=0$ can be achieved, cf. [MR03, p. 73].
} 
In [PR88, section III], Pohlmeyer and Rehren showed that the invariant charges are complete in the sense that for a certain class of worldsheets, the string's worldsheet can be reconstructed uniquely up to global translations in spacetime from $\mathfrak{h}$.

If we expand the power series of the logarithm of the monodromy matrix instead of the power series of the monodromy matrix itself, we obtain the so-called homogeneous tensors, in particular those of homogeneity degree 1 , which are called the truncated tensors $\mathbb{R}^{4}$.

Since equations (3.5) and (3.6) mirror equation (1.33), and we had already identified the tensors $\mathcal{R}$ with words, we can identify homogeneous tensors with (higher) Euler idempotents of words and in particular the truncated tensors with Euler elements.

From the construction of the different types of tensors introduced in this section, several relations between them can be deduced. The tensors $\mathcal{R}$ can be written as a linear combination of homogeneous tensors (of degrees $k$ ) with the same index word,

$$
\mathcal{R}_{x_{1} \ldots x_{n}}=\sum_{k=1}^{n} \mathcal{R}_{x_{1} \ldots x_{n}}^{(k)}
$$

while the homogeneous tensors of degree $k>1$ are called that way because they are homogeneous polynomials of degree $k$ in the truncated tensors:

$$
\mathcal{R}_{x_{1} \ldots x_{n}}^{(k)}=\frac{1}{k !} \sum_{0<i_{1}<\ldots i_{k-1}<n} \mathcal{R}_{x_{1} \ldots x_{i_{1}}}^{t} \cdot \mathcal{R}_{x_{i_{1}+1} \ldots x_{i_{2}}}^{t} \cdot \ldots \cdot \mathcal{R}_{x_{i_{k-1}+1} \ldots x_{n}}^{t} \cdot
$$

Remark 3.1.1. Historically, the Poisson bracket (3.4) on the invariants originally was arrived at as a Poisson bracket induced by the Poisson bracket on the space spanned by the left and right movers. That Poisson bracket was in turn induced by the one on the canonical coordinates of position and momentum. When extending the original bracket to the truncated tensors (Euler elements), one obtains an antisymmetric bilinear map which does not satisfy the Jacobi equation. Under cyclic summation however, the terms violating the Jacobi equation cancel out. Removing those terms form the Poisson bracket yields what was originally called the "modified Poisson bracket" and which is called the Pohlmeyer-Rehren Lie bracket (1.54) here. Because the removed terms vanish under the cyclic summation, derivative extension as discussed above (resulting in (3.4) ) leads back to the original Poisson bracket.

Remark 3.1.2. We have now collected all the facts to give a list of reasons why $d=3$ and $d=4$ receive special consideration in this thesis. Since $\operatorname{dim} \mathfrak{g}_{l}=\operatorname{NumLyndon}(l+2, d)$, which is monotonously increasing with $d$ for a given $l$ (and an upper bound of which is given $d^{l+2}$ ), cases with lower $d$ are generally considerably more manageable.

- One major reason lies in the application to the quantization of the Nambu-Goto string.

- Since $d$ is the dimension of the string's surrounding spacetime and we apparently live in a four-dimensional spacetime, the case of $d=4$ is important to describe strings in the actual physical universe. This is especially important since the Pohlmeyer approach has the distinction of having no known obstruction to quantization in this spacetime dimension, unlike the usual conformal field theory methods of string quantization which require a critical dimension of $d=26$ (or $d=10$ with supersymmetry).

\footnotetext{
${ }^{4}$ In [MR03], they are called "monodromy variables".
} 
$-d=3$ can serve as a simpler toy model.

- Even lower dimensions are less well suited for this; for $d=2$, the preimage of almost every point on any (spacelike) closed curve parametrizing the string for a given parameter $\tau$ has at least two elements, in contrast to the usual picture of a simple (i.e. injective except for the points on the boundary of the parametrizing interval) curve, so degeneracy is to be expected.

- For $d=1$, no linearly independent time and space dimensions exist.

- The structure of $\mathfrak{g}$ itself, in particular as a $\mathfrak{g}_{0} \cong \mathfrak{s} \mathfrak{d}(d, \mathbb{C}$ )-module (cf. remark 2.2.15) gives further reasons:

- For $d=1$, there are no Lyndon words of more than a single letter, therefore $\mathfrak{g}$ is the one-dimensional Lie algebra spanned by $e(0) \in \mathfrak{g}_{-1}$, and $\mathfrak{g}_{0}=0$.

- For $d=2$, the stratum $\mathfrak{g}_{0} \cong \mathfrak{s}(2, \mathbb{C})$ is one-dimensional, hence abelian, and all irreducible $\mathrm{g}_{0}$-modules are one-dimensional as well. Additionally, it is known that $\mathfrak{g}_{1}$ does not generate $\mathfrak{g}_{\geq 1}$ (cf. proposition 1.5.3), while the opposite is conjectured for $d \geq 3$ (conjecture 4.1.2).

- So $d=3$ is the lowest dimension in which a rich representation theory (of $\left.\mathfrak{g}_{0} \cong \mathfrak{s} \mathfrak{D}(3, \mathbb{C}) \cong \mathfrak{s l}_{2}\right)$ develops.

- Regarding its representation theory, $d=4$ is an unusual case since it is the only dimension for which $\mathfrak{g}_{0} \cong \mathfrak{s}(4, \mathbb{C}) \cong \mathfrak{s l}_{2} \times \mathfrak{s l}_{2}$ is nonsimple. Connected to this is the fact that the ladder operators commute and for all weights, the multiplicity of every weight $\mu \in \Gamma$ (cf. remark 2.3.7) is $n(\mu)=1$.

\subsection{Poisson algebra of invariant charges in the shuffle Hopf lan- guage}

\subsubsection{Induced Poisson structure $\mathfrak{i}$}

In the sequel, we will use shuffle Hopf algebra language again. In order to do that, we have to catch up in notation and translate the concept of invariant charges, mostly following the accounts given in [Mei09] and [BM11] throughout this section again.

We have two multiplications on the shuffle algebra - the shuffle product (definition 1.1.22), which is commutative and associative, and the Pohlmeyer-Rehren Lie bracket (definition 1.4.3). We can now extend the Lie bracket derivatively to shuffle products of Euler elements, i.e.

$$
\begin{aligned}
\{e(x), e(y)\} & :=[e(x), e(y)], \\
\{e(x) \# e(y), e(z)\} & :=e(x) \#\{e(y), e(z)\},+\{e(x), e(z)\} \# e(y)
\end{aligned}
$$

for $x, y, z \in X^{*} .5$

\footnotetext{
${ }^{5}$ Cf. [Mei09, p. 50f]. There, it is not explicitly pointed out that this is well-defined because there are no multiplicative relations by theorem 1.2.2 4 - otherwise, it would have to be proved that rewriting the left hand side using such a relation could not lead to ambiguities.
} 
Remark 3.2.1. Relations (1.33) and (1.34) imply that

$$
\operatorname{Sh}(X)=\operatorname{span}_{\mathbb{K}}\left(e\left(x_{1}\right) \# \ldots \# e\left(x_{k}\right) \mid k \in \mathbb{N}_{0}, x_{i} \in X^{*} \forall i \in\{1, \ldots, k\}\right)
$$

as sets, and they can be used to calculate the Poisson brackets of two words. To calculate $\{x, y\}$ for words $x, y$ of lengths $n$ and $m$ respectively (cf. [Mei09, p. 51]),

1. rewrite $x$ and $y$ in terms of sums of shuffle products of Euler elements using equation (1.33), and use linearity of the Poisson bracket, yielding

$$
\{x, y\}=\sum_{k=1}^{n} \sum_{l=1}^{m} \frac{1}{k !} \frac{1}{l !} \sum_{\substack{x_{1}, \ldots, x_{k} \in X^{*} \backslash X^{0} \text { s.t. } \\ x_{1} \sim \ldots \smile x_{k}=x}} \sum_{\substack{y_{1}, \ldots, y_{l} \in X^{*} \backslash X^{0} \\ y_{1} \sim \ldots . t .}}\left\{e\left(x_{1}\right) \# \ldots \# e\left(x_{k}\right), e\left(y_{1}\right) \# \ldots \# e\left(y_{l}\right)\right\}
$$

2. use Leibniz's rule to move all shuffle products out of the brackets (since \# is commutative, we can collect all factors on one side),

$$
\begin{aligned}
& \ldots=\sum_{k=1}^{n} \sum_{l=1}^{m} \frac{1}{k !} \frac{1}{l !} \sum_{\substack{x_{1}, \ldots, x_{k} \in X^{*} \backslash X^{0}=\text { s.t. } \\
x_{1} \sim \ldots \sim x_{k}=x}} \sum_{\substack{y_{1}, \ldots, y_{l} \in X^{*} \backslash X^{0} \\
y_{1} \sim \ldots \sim y_{l}=y}} \sum_{\text {s.t. }}^{k} \sum_{i=1}^{l}\left\{e\left(x_{i}\right), e\left(y_{j}\right)\right\} \\
& \# e\left(x_{1}\right) \# \ldots \# e\left(x_{i-1}\right) \# e\left(x_{i+1}\right) \# \ldots \# e\left(x_{k}\right) \# e\left(y_{1}\right) \# \ldots e\left(y_{j-1}\right) \# e\left(y_{j+1}\right) \# \ldots \# e\left(y_{l}\right) \text {, }
\end{aligned}
$$

3. evaluate the remaining brackets of the form $\{e(v), e(w)\}$, leaving us with a linear combination of shuffles of Euler elements

4. re-write all the Euler elements in terms of words using equation (1.34), so we obtain a linear combination of shuffles of words

5. and finally evaluate the shuffles, which leaves us with the desired linear combination of words.

Theorem 3.2.2. $\quad$ 1. $(\operatorname{Sh}(X), \#,\{\cdot, \cdot\})$ is a Poisson algebra, henceforth to be designated $i$.

2. $i$ is graded by the combined degree defined as

$$
\operatorname{deg}\left(e\left(x_{1,1} \ldots x_{1, n_{1}}\right) \# \ldots \#\left(x_{k, 1} \ldots x_{k, n_{k}}\right)\right):=-k-1+\sum_{i=1}^{k} n_{i},
$$

and with $\mathfrak{i}^{l}:=\{i \in \mathfrak{i} \mid \operatorname{deg} i=l\}$, we have

$$
\begin{aligned}
\left\{\mathfrak{i}^{l}, \mathfrak{i}^{l^{\prime}}\right\} & \subset \mathfrak{i}^{l+l^{\prime}}, \\
\mathfrak{i}^{l} \# \mathfrak{l}^{l^{\prime}} & \subset \mathfrak{i}^{l+l^{\prime}+1} .
\end{aligned}
$$

Proof. 1. $(\operatorname{Sh}(X), \#,\{\cdot, \cdot\})$ inherits the axioms for the Poisson bracket from the Lie algebra g; compatibility with the shuffle product is by construction (cf. [Mei09][p. 50]).

2. can easily be proved by counting letters (this is [Mei09][Satz 4.8]). 


\subsubsection{Poisson algebra of invariant charges $\mathfrak{h}$}

The Poisson algebra of invariant charges can be constructed in the shuffle Hopf algebra language in an analogous way to equation (3.3).

Theorem 3.2.3 (Poisson algebra of invariant charges). Let $Z: \operatorname{Sh}(X) \rightarrow \operatorname{Sh}(X)$ be the cyclic symmetrization map, i.e. for all words $x_{1} \ldots x_{n} \in X^{*}$

$$
\left.Z\left(x_{1} \ldots x_{n}\right):=\sum_{i=1}^{n} x_{i} \ldots x_{n} x_{1} \ldots x_{i-1} \quad\left(=\mathcal{Z}_{x_{1}, \ldots x_{n}}\right) \text { in PohLMEYeR's notation }\right) .
$$

Then

$$
\mathfrak{h}:=\operatorname{im} Z \subset \mathfrak{i}(=\operatorname{Sh}(X))
$$

is a Poisson subalgebra of $\mathfrak{i}$ (that inherits the gradation from $\mathfrak{i}$ ) and is called the Poisson algebra of invariant charges.

Proof. This is [Mei09, Satz 4.9], based on [PR86, Proposition 12].

We have thus compiled a small dictionary of terms in Pohlmeyer's original language and

\begin{tabular}{|c|c|c|c|}
\hline original term & symbol & shuffle Hopf language term & symbol \\
\hline $\begin{array}{l}\text { tensor } \\
\text {... with cyclically minimal indices }\end{array}$ & $\mathcal{R}_{x_{1}, \ldots, x_{n}}$ & $\begin{array}{l}\text { word } \\
\text { Lyndon word }\end{array}$ & $x_{1} \ldots x_{n} \in X^{n}$ \\
\hline truncated tensor & & Euler element & $e\left(x_{1} \ldots x_{n}\right)$ \\
\hline invariant charge & $\mathcal{Z}_{x_{1}, \ldots x_{n}}$ & invariant charge & $Z\left(x_{1} \ldots x_{n}\right)$ \\
\hline tensorial multiplication & - (or omitted) & shuffle product & \# (or omitted) \\
\hline modified Poisson bracket & {$[\cdot, \cdot]$} & Pohlmeyer-Rehren Lie bracket & {$[\cdot, \cdot]$} \\
\hline
\end{tabular}
their analogues in the shuffle Hopf algebra language.

Table 3.1: Corresponding terms in the languages used in PoHLMEYeR's original formulation and the reformulation in terms of the shuffle Hopf algebra due to BaHNs and Meinecke.

Remark 3.2.4. For the same reason (lemma 2.2.2) that $\mathfrak{g}$ is a $\mathfrak{g}_{0}$-module, the Poisson algebra of invariant charges $\mathfrak{h}$ is a $\mathfrak{h}_{0}$-module, considering $\mathfrak{h}_{0}$ as a Lie subalgebra $\mathfrak{6}^{6}$ It was shown that $\mathfrak{h}_{0} \cong \mathfrak{s} \mathfrak{v}(d-1, \mathbb{C})$. Therefore, many of the features familiar from $\mathfrak{g}$ such as the decomposition into irreducible modules (multiplets) also occur in $\mathfrak{h}$. Note however, that for a given $d$, $\mathfrak{h}_{0} \cong \mathfrak{s} \mathfrak{v}(d-1, \mathbb{C}) \varsubsetneqq \mathfrak{s} \mathfrak{d}(d, \mathbb{C}) \cong \mathfrak{g}_{0}$.

\subsubsection{The rest frame}

A consequence [Poh99, p. 3] of definig equation (3.1) is that the Euler idempotents of singleletter words are the components of the total $d$-dimensional momentum $P$ of the string. In fact, we can write

$$
e(x)=x=\int u\left(\sigma_{1}, \tau\right) d \sigma_{1}=\int p_{x}\left(\sigma_{1}, \tau\right) d \sigma_{1}=P_{x} \quad \forall x \in X .
$$

\footnotetext{
${ }^{6}$ Note that $\mathfrak{h}_{0}$ is not a Poisson subalgebra of $\mathfrak{h}$ since the shuffle product is of degree +1 (cf. equation $(3.12)$ ).
} 
We can now distinguish two cases depending on the Lorentz square $P^{2}:=v_{x y} P_{x} P_{y}$ of the string. If $P^{2}=0$, we are in the massless case. If $m^{2}:=P^{2}>0$, we are in the massive case and call $m$ the string's invariant mass. Because the theory is much more developed for massive strings, we will only deal with this case.

Since the Poincaré group acts on $\mathfrak{h}$, leaving the combined degree $l$ invariant, we can use a Lorentz transformation to the string's relativistic rest frame, and in this situation, $e(0)=m$ is the string's invariant mass while $e(x)=0$ for all $x \in X \backslash\{0\}$. A useful consequence of this is that in expressions of invariant charges as Euler-Lyndon words, many terms disappear, for instance

$$
Z\left(e^{(2)}(012)\right)=2 m e(1,2)
$$

We designate the Poisson algebra of invariants for the string's rest frame by $\mathfrak{h}_{m}$. Having this simplification at our disposal, we can now turn our attention to some statements about the structure of $\mathfrak{h}_{m}$.

\subsubsection{Generation of $\mathfrak{h}_{m}$ as a Poisson algebra}

Theorem 3.2.5 (standard invariants). The elements of $\mathfrak{h}_{m}$

$$
\begin{aligned}
Z\left(e^{(2)}(0 a b)\right) & \in \mathfrak{h}_{m}^{0}, \\
\left.\frac{1}{K} Z\left(e^{(2)}\left(0 a 0^{K-1} b\right)\right)\right) & \in \mathfrak{h}_{m}^{K-1}, \\
(K-1) ! Z\left(e^{(K)}\left(0^{K-1} a x_{1} \ldots x_{K-1} b\right)\right) & \in \mathfrak{h}_{m}^{K-1}
\end{aligned}
$$

for $K>2$, where $a, b \in X \backslash\{0\}$ and $x_{i} \in X$ for all $i$ as well as $x_{i} \neq 0$ for at least one $i$, freely generate $\mathfrak{h}_{m}$ as an algebra (using shuffle multiplication). These generators are called the standard invariants.

\section{Proof. See [PR86, Proposition 17]}

Unlike for the shuffle multiplication, there are relations between (multiple) Poisson brackets of the standard invariants. A large subalgebra of $\mathfrak{h}_{m}$, denoted by $\mathfrak{d}$, is the subalgebra generated - as a Poisson algebra - by the elements of $\mathfrak{h}_{m}^{0}$ and $\mathfrak{h}_{m}^{1}$. But not all elements of $\mathfrak{h}_{m}$ are contained in $\mathfrak{U}$.

Definition 3.2.6 (exceptional element). An element of $\mathfrak{h}_{m}^{l}$ with $l \geq 2$ that is linearly independent from all (multiple) Poisson brackets of standard invariants of degree $<l$ is called exceptional element.

The subspace spanned by the exceptional elements is infinite-dimensional:

Proposition 3.2.7. The invariants

$$
L_{(1)}:=\sum_{\mu, v} g_{\mu v} \mathcal{Z}\left(e_{0 \mu 0^{l} v}^{(2)}\right)
$$

with $l=2 n+1, n \in \mathbb{N}_{+}$are exceptional elements. 
Proof. Pohlmeyer and Rehren's proof (see [PR86, p. 622]) works by considering the leading term, which is a multiple of

$$
\sum_{i=1}^{d-1} e(0) \# e(i 0 \ldots 0 i),
$$

the right hand factor of which is an element of the Lie subalgebra

$$
\operatorname{ker} \partial_{0}^{R} \cap \operatorname{ker} \partial_{0}^{L}=\operatorname{span}\left(x \in X^{*} \text { with } x=1_{\emptyset} \text { or } x_{1}=x_{n}=0\right)
$$

of $\mathfrak{g}$, and showing that this term cannot be produced as a linear combination of leading terms of other invariants.

All known exceptional elements could be modified by adding Poisson brackets of standard invariants such that the resulting modified exceptional elements commute with each other. This has lead to the following conjecture (cf. [Poh99, p. 8ff]):

\section{Conjecture 3.2.8.}

$$
\mathfrak{h}_{m}=\mathfrak{a} \ltimes \mathfrak{U},
$$

where $\mathfrak{a}$ is an abelian Lie algebra spanned by modified exceptional elements.

Since all known exceptional elements as well as the generators of $\mathfrak{U}$ have homogeneity degree 2, an additional conjecture was reached.

Conjecture 3.2.9 (Quadratic generation hypothesis). As Poisson algebras, $\mathfrak{h}_{m}$ as well as $\mathfrak{h}$ are generated by their elements of homogeneity degree 2 (also called "quadratic elements").

The quadratic generation hypothesis is not only interesting in its own right; it will be of great importance to the ReHREN-MeINECKE approach to string quantization that will be discussed in the next section.

Remark 3.2.10. For $d=3$, all relations between elements of $\mathfrak{a} \ltimes \mathfrak{H}$ up to degree $l=5$ have been computed [Hap93] by K. M. HAPpLE using computer algebra, applying Hall bases similar to the ones given in chapter 4

For $d=3$, a basis of $\mathfrak{h}_{m 0}$ is given by the element

$$
Q:=\frac{\mathbf{i}}{2 m} \mathcal{Z}_{0-+}^{(2)}=\frac{\mathbf{i}}{2} e(-+),
$$

and a basis of $\mathfrak{h}_{m 1}$ is given by the elements

$$
\begin{aligned}
& L_{2}:=\quad \mathcal{Z}_{00++}^{(2)}=\quad 2 m e(0++)-(e(0+))^{2}, \\
& L_{1}:=\quad-\sqrt{2} Z_{0-++}^{(2)}=\quad-\sqrt{2}(m e(-++)-e(0+) e(-+)),
\end{aligned}
$$

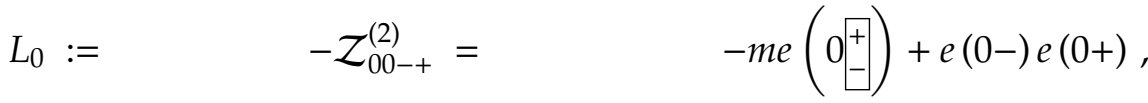

$$
\begin{aligned}
& L_{-1}:=\quad-\sqrt{2} Z_{0+--}^{(2)}=\quad \sqrt{2}(m e(--+)-e(0-) e(-+)) \text {, } \\
& L_{-2}:=\quad \mathcal{Z}_{00--}^{(2)}=\quad 2 m e(0--)-(e(0-))^{2}
\end{aligned}
$$

(this is [Hap93, Korollar 7.2]).

More on HAPPLE's work and its relationship to the algorithms developed in chapter $4 \mathrm{can}$ be found in remark 4.3.14. 
Remark 3.2.11. The exceptional elements $L_{(1)}$ given in equation (3.16) are not the only exceptional elements. Pohlmeyer also pointed out in his last publication [Poh06, p.3] (without a proof) that an exceptional element with leading term

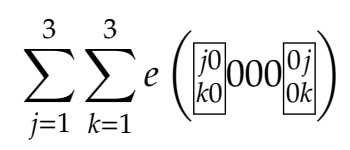

(for $d=3+1$ ) exists and conjectured that it was "probably" the first in a new infinite series of exceptional elements.

\subsection{Quantization}

\subsubsection{Pohlmeyer's original programme}

PohlmeYer's original approach to quantization [Poh99, p. 19ff] was to quantize $\mathfrak{h}_{m}$ without reference to other quantities. This is done by finding generators of $\mathfrak{h}_{m}$ and assigning to each of them one of the free generators of a filtered associative (noncommutative) algebra. The desired quantum algebra of invariants $\hat{\mathfrak{h}}$ is then constructed by dividing out an ideal of relations. The relations forming the ideal are constructed by deforming the classical relations such that quantization conditions are met, i.e. $\mathfrak{h}_{m}^{l} \cong \hat{\mathfrak{h}}_{m}^{l} / \hat{\mathfrak{h}}_{m}^{l-1}$ as vector spaces and the Poisson bracket is reproduced as a commutator except for quantum corrections of lower degree with positive powers of a formal parameter $h$. Similarly, the relations of $\mathfrak{h}$ are recovered in the quasiclassical limit $h \rightarrow 0$. These deformations are calculated by adding a linear combination of all possible (i.e. satisfying conditions such as on degree, spin, parity, degree in $h$ ) quantum corrections to a given relation and calculating their coefficients by performing consistency checks.

While this method has produced some valuable insights into the quantization of the Poisson algebra of invariants, it is very laborious and in principle unable to actually prove the existence of a quantization because it works stratum by stratum and therefore cannot rule out the possibility of contradictions in strata not considered yet.

\subsubsection{Meusburger-Rehren quantization}

The approach to the quantization of the Poisson algebra of invariant charges $\mathfrak{h}$ proposed by Rehren to Pohlmeyer and fleshed out by Meusburger in her Diplomarbeit [Meu01] starts from the fact that $\mathfrak{h}$ can be understood as the kernel of a derivation $7 \partial$. We continue following [BM11].

Theorem 3.3.1 (Poisson algebra of invariants as kernel of a derivation). 1. The linear map

$$
\begin{aligned}
\partial: \mathfrak{i} & \rightarrow \mathfrak{i} \otimes \mathfrak{i}, \\
x_{1} \ldots x_{n} & \mapsto \begin{cases}x_{1} \otimes\left(x_{2} \ldots x_{n}\right)-x_{n} \otimes\left(x_{1} \ldots x_{n-1}\right) & \text { if } n \geq 2 \\
0 & \text { else }\end{cases}
\end{aligned}
$$

for $x_{1}, \ldots, x_{n} \in X$ is a derivation along the morphism $f: \mathfrak{i} \rightarrow \mathfrak{i} \otimes \mathfrak{i}, \quad x \mapsto 1 \otimes x$.

\footnotetext{
${ }^{7}$ Originally[Meu01, Kap. 6 Lemma 1], a variant of the restriction of $\partial$ to im $e$ from statement 2 of theorem 3.3.1 was used. The principle is the same though, and the proofs are analogous.
} 
2. $\partial$ can be restricted to ime, obtaining

$$
e\left(x_{1} \ldots x_{n}\right) \mapsto \begin{cases}e\left(x_{1}\right) \otimes\left(e\left(x_{2} \ldots x_{n}\right)\right)-e\left(x_{n}\right) \otimes\left(e\left(x_{1} \ldots x_{n-1}\right)\right) & \text { if } n \geq 2 \\ 0 & \text { else }\end{cases}
$$

for $x_{1}, \ldots, x_{n} \in X$.

3.

$$
\mathfrak{h}=\operatorname{ker} \partial \subset \mathfrak{i} .
$$

Proof. This is [Mei09, Satz 4.10 and Satz 4.12] (using the recursive formula for the shuffle product 1.1.4.

To quantize $\mathfrak{g}$, we first introduce a formal variable $h$ and define $\mathfrak{g}_{h}$ as the Lie algebra obtained by equipping $\mathfrak{g}[[h]]$ (the ring of formal power series with coefficients in the Lie algebra $\mathfrak{g}$ in the variable $h$ ) with the Lie bracket defined like the Lie bracket on $\mathfrak{g}$, modified by multiplying the structure constants by global factor $h$.

The idea is to then construct a quantized algebra of invariants $\hat{\mathfrak{h}}$ in a similar fashion to theorem 3.3.1 as the kernel of another derivation $\delta$ in the universal enveloping algebra $U\left(\mathfrak{g}_{h}\right)$,

$$
\hat{\mathfrak{h}}=\operatorname{ker} \delta \subset U\left(\mathfrak{g}_{h}\right) .
$$

This is done in such a way that in the quasiclassical limit, $h \rightarrow 0$, the classical Poisson algebra of invariants is recovered. This concept reappears in the formalized notion of a deformation of a Poisson algebra (definition 3.3.5). But first, we should recall the definition of the universal enveloping algebra of a Lie algebra.

Definition 3.3.2 (universal enveloping algebra). Let $G$ be a Lie algebra, $U$ an unital associative algebra (both over the field $\mathbb{K}$ ) and $\phi: G \rightarrow U$ be homomorphism of Lie algebras (considering $U$ with the Lie bracket defined by the commutator

$$
[x, y]:=x y-y x \forall x, y \in U
$$

as a Lie algebra). Let further $(U, \phi)$ satisfy the following universal property: for any unital associative $\mathbb{K}$-algebra $A$ and homomorphism of Lie algebras $\phi^{\prime}: G \rightarrow A$ exists a unique homomorphism of algebras $\psi: U \rightarrow A$ such that the diagram

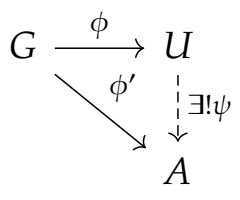

commutes. Then $U$ is called an universal enveloping algebra of $G$.

By the usual argument for universal objects, the universal enveloping algebra is unique (up to isomorphisms), and since it can be constructed as a quotient algebra of the tensor algebra $T(G)$ modulo the ideal generated by elements of the form $x \otimes y-y \otimes x-[x, y]$ (which guarantees that the quotient satisfies equation (3.29), it exists. This justifies calling $U$ the universal enveloping algebra of the Lie algebra $G$ and writing $U(G)$ for the universal enveloping algebra of a Lie algebra $G$. 
If the Lie algebra $G$ is graded, then $U(G)$ inherits some of that structure:

$$
U(G)_{l}:=\operatorname{span}\left\{x_{1} \cdot \ldots \cdot x_{k} \mid x_{i} \in G, 0 \leq \sum_{i} \operatorname{deg}\left(x_{i}\right)+k-1 \leq l\right\}
$$

is a filtration on $U(G)$ with the degree

$$
\operatorname{deg}(u):=\min \left(l \mid u \in U(G)_{l}\right) \text { for } u \in U(G) .
$$

From this definition, one can infer that the multiplication in $U(G)$ is of degree +1 and the commutator is of degree 0 :

$$
\begin{aligned}
U(G)_{l} \cdot U(G)_{l^{\prime}} & \subset U(G)_{l+l^{\prime}+1}, \\
{\left[U(G)_{l}, U(G)_{l^{\prime}}\right] } & \subset U(G)_{l+l^{\prime}} .
\end{aligned}
$$

The fact that the two sides of equation (3.29) now have different degrees explains why we have only equipped $U(G)$ with a filtration instead of a gradation.

When considering the universal enveloping algebra of $\mathfrak{g}_{h}$, note that the multiplication is no longer the (commutative) shuffle product, but the non-commutative multiplication in $U\left(\mathfrak{g}_{h}\right)$ with the property that the Lie bracket of $\mathfrak{g}$ is mapped to the commutator.

If a basis of a Lie algebra is known, a basis of its universal enveloping algebra is given by the Poincaré-Birkhoff-Witt theorem.

Theorem 3.3.3 (Poincaré-Birkhoff-Witt theorem). Let $G$ be a Lie algebra with basis $\left\{x_{i}\right\}_{i \in I}$, where I is a totally ordered index set. Then the set

$$
\left\{\phi\left(x_{i_{1}}\right) \cdot \ldots \cdot \phi\left(x_{i_{n}}\right) \mid i_{1} \leq \ldots \leq i_{n}\right\}
$$

is a basis of $U(G)$.

Proof. See for instance [Hum72, section 17.4].

In particular, all monomials in $U(G)$ can be reordered using equation 3.29 , and the result is independent of the sequence in which the different factors of a monomial are reordered.

\subsubsection{Quantization of Poisson algebras}

This shift from commutative to non-commutative situations is typical of quantization in physics and can be formalized using the following notions. We continue following [BM11], which adapts definitions and methods from [ES09, chapter 1].

Definition 3.3.4 (h-adic topology, topologically free module). $\quad 1$. Let $\mathbb{K}$ be a field. We denote by $K:=\mathbb{K}[[h]]$ (the ring of formal power series with coefficients in $\mathbb{K}$ in the variable $h$ ). In this situation $K$ is equipped with the h-adic norm defined by

$$
\left\|a_{n} h^{n}+a_{n+1} h^{n+1}+\ldots\right\|:=C^{-n} \quad\left(a_{n} \neq 0\right),
$$

where $C>1$ is any fixed constant. $K$ is complete with respect to the $h$-adic norm, and the topology defined by the $h$-adic norm is called the $h$-adic topology. 
2. Let $V$ be a vector space over a field $\mathbb{K}$. Define

$$
V[[h]]:=\left\{\sum_{n=0}^{\infty} v_{n} h^{n} \mid v_{n} \in V\right\} .
$$

Now, the $h$-adic norm and topology on $V[[h]]$ can be defined analogously to 1 .

3. A topological $K$-module (i.e. a $K$-module with a topology such that addition and scalar multiplication are continuous) that is isomorphic to $V[[h]]$ for some $\mathbb{K}$-vector space $V$ is called a topologically free K-module.

Definition 3.3.5 (deformation algebra, deformation). 1. A topologically free $K$-algebra, i.e. a topologically free $K$-module $A$ with a $K$-bilinear map $A \times A \rightarrow A$ such that $A$ with this map is an associative algebra is called a deformation algebra.

2. Let $A_{c}$ be an associative $\mathbb{K}$-algebra. A deformation algebra $A$ such that $A_{c}=A / h A$ is called deformation of $A_{c}$.

Definition 3.3.6 (quantization, quasiclassical limit). Let $A_{c}$ be an associative commutative $\mathbb{K}$-algebra with deformation algebra $A$. Let $f, g \in A_{c}$ with arbitrary liftings $\hat{f}, \hat{g} \in A$. Because $A_{c}$ is commutative, the commutator on $A_{c}$ identically vanishes, but one can recover a similar structure on $A_{c}$ from $A$ : define

$$
\{f, g\}:=\frac{1}{h}(\hat{f} \cdot \hat{g}-\hat{g} \cdot \hat{f}) \quad(\bmod h) \quad \in A_{c},
$$

where $\cdot$ is the (not necessarily commutative) multiplication on $A$. Then $\left(A_{c},\{\},\right)$ is a Poisson algebra, called the quasiclassical limit of $A$ and $A$ is called a quantization of $A_{c}$.

These definitions encode the physical concept that a formal parameter $h$ is introduced in such a way that in the quasiclassical limit (in the informal sense of) $h \rightarrow 0$, the commutators (having $h$ as a factor) vanish. Such a vanishing of commutators in the quasiclassical limit is typical for the relationship between physical quantum observables and their classical counterparts.

As the elements of $U(\mathfrak{g})_{l}$ can be understood as the non-commutative polynomials of degree $l$, the elements of $S(\mathfrak{g})_{l}$ are the commutative polynomials of degree $l$, and because the commutators are of lower degree with respect to the filtration, we obtain the quotient $U(\mathfrak{g})_{l} / U(\mathfrak{g})_{l-1} \cong S(\mathfrak{g})_{l}$. We will use $A_{c}:=S(\mathfrak{g})$, with the same filtration. $A_{c}$ not only carries the multiplication from $S(\mathfrak{g})$, but also the derivative extension of the Lie bracket from $\mathfrak{g}$, making $A_{c}$ a Poisson algebra.

To construct a quantization of $A_{c}$, we consider the topologically free algebra

$$
\begin{aligned}
\mathcal{A}_{\mathfrak{g}} & :=\left(\widehat{\bigoplus}_{l \geq 0} h^{l} U\left(\mathfrak{g}_{h}\right)_{l}\right)[[h]] \\
& :=\left\{\sum_{i \in \mathbb{N}_{0}} h^{i} v_{i} \mid v_{i} \in U\left(\mathfrak{g}_{h}\right) \text { s.t. } i \geq \operatorname{deg}\left(v_{i}\right) \forall i \in \mathbb{N}_{0}, i-\operatorname{deg}\left(v_{i}\right) \stackrel{i \rightarrow \infty}{\rightarrow} \infty\right\}
\end{aligned}
$$

and the $\operatorname{map} \varphi: \mathcal{A}_{\mathfrak{g}} \rightarrow A_{c}$

$$
\sum h^{i} v_{i} \mapsto \sum_{j=0}^{\infty} p \circ \operatorname{lead}_{j}\left(v_{j}\right)
$$


where lead ${ }_{j}\left(v_{j}\right)$ is the leading order contribution of $v_{j}$, i.e. the contribution with degree equal to $j$, and $p$ is the projection $\mathcal{A}_{\mathfrak{g}} \rightarrow A_{c}$. $\varphi$ is well defined because the sum on the right hand side is actually finite since $\operatorname{deg}\left(v_{j}\right)<j$ for almost all $j$ by defining equation (3.38). Now, as desired, $\mathcal{A}_{\mathrm{g}}$ is a quantization of $A_{c}$ because by construction $\operatorname{ker} \varphi=h \mathcal{A}_{\mathrm{g}}$.

Given an element $X \in A_{c}^{l}$, a lifting in $\mathcal{A}_{\mathfrak{g}}$ of the form

$$
\left(h^{l} X\right)+h\left(h^{l-1} Y_{l-1}\right)+\ldots+h^{l-1}\left(h Y_{1}\right)+h^{l}\left(Y_{0}\right)
$$

with $Y_{l^{\prime}} \in U\left(\mathfrak{g}_{h}\right)_{l^{\prime}}$ is called a quantum counterpart for $X$ while the summands from lower strata are called the quantum corrections of $X$.

\subsubsection{Construction of the Meusburger-Rehren quantization}

To construct the derivation $\delta$ intended to have a quantized algebra of invariants as its kernel (cf. p 78), we begin with an action.

\section{Lemma 3.3.7.}

The map

$$
\begin{aligned}
\mathfrak{g} \times \dot{\mathfrak{i}}_{-1} & \rightarrow \mathfrak{i}_{-1}, \\
(e(x), c) & \mapsto e(x) \cdot c:= \begin{cases}g_{a c} b-g_{b c} a & \text { if } x=a b \text { with } a, b \in X \\
0 & \text { if } x \notin X^{2}\end{cases}
\end{aligned}
$$

defines an action of $\mathfrak{g}$ on $\mathfrak{i}_{-1}$ (by defining equation 3.10 the one-letter words in $\operatorname{Sh}(X)$ ).

Proof. This is [MR03][Proposition 1 part 1].

This action now defines the semidirect product $\mathfrak{g}_{h} \rtimes \mathfrak{i}_{-1}$, the universal enveloping algebra of which will be the codomain of the desired derivation $\delta$.

Theorem 3.3.8. $\quad$ 1. The linear map

$$
\begin{aligned}
\delta: \quad \mathfrak{g}_{h} \rightarrow U\left(\mathfrak{g}_{h} \rtimes \mathrm{i}_{-1}\right), & \\
e\left(x_{1} \ldots x_{n}\right) & \mapsto \begin{cases}{\left[v_{x_{1}}, e\left(x_{2} \ldots x_{n}\right)\right]_{+}-\left[v_{x_{n}}, e\left(x_{1} \ldots x_{n-1}\right)\right]_{+}} & \text {if } n \geq 2 \\
0 & \text { else }\end{cases}
\end{aligned}
$$

(where $v_{0}, \ldots, v_{d-1}$ are basis vectors of $i_{-1}$ and $[x, y]_{+}:=\frac{1}{2}(x y+y x)$ is the anticommutator) is a derivation of Lie algebras.

2. Its (unique) extension $\delta: U\left(\mathfrak{g}_{h}\right) \rightarrow U\left(\mathfrak{g}_{h} \rtimes \mathrm{i}_{-1}\right)$ to the universal enveloping algebra $U\left(\mathfrak{g}_{h}\right)$ is a derivation with the property that

$$
\operatorname{im} Z \circ e^{(2)} \subset \operatorname{ker} \delta \subset U\left(\mathfrak{g}_{h}\right) .
$$

Proof. 1. See [BM11][Proposition 12]. This statement as well as the proof is based on [MR03][Proposition 1 part 1] but uses a slightly different derivation.

2. This is covered by [BM11][Proposition 12] as well. 
Remark 3.3.9. Note that the above theorem 3.3.8 is weaker than its classical analogue 3.3.1 in that only the quadratic quantum invariants are contained in the kernel of the derivation $\delta$ while all classical invariants are in the kernel of $\partial$, so that the postulate formulated in equation (3.28) is not met in its generality. In fact, Meusburger [Meu01][p. 63] demonstrated that the invariant (of homogeneity degree 3) $Z\left(e^{(3)}(001101)\right.$ ) does not lie in the kernel of $\delta$.

Corollary 3.3.10 (quantization). We can assign to each quadratic invariant $Z\left(e^{(2)}\left(x_{1} \ldots x_{n}\right)\right) \in \mathfrak{h}^{n-3}$ a quantized invariant

$$
\hat{Z}^{(2)}\left(x_{1} \ldots x_{n}\right):=h^{n-3} Z\left(\frac{1}{2} \sum_{i=1}^{n-1} e\left(x_{1} \ldots x_{i}\right) e\left(x_{i+1} \ldots x_{n}\right)\right) \in h^{n-3} U\left(\mathfrak{h}_{h}\right)_{n-3} .
$$

By theorem 3.3.8. $\hat{Z}^{(2)}(x) \in \operatorname{ker} \delta \subset \mathcal{A}_{\mathfrak{g}}$ for any word $x \in X^{*}$. If the quadratic generation hypothesis (conjecture 3.2.9) is true, then $\varphi$ maps ker $\delta$ surjectively to $\mathfrak{h}$ and

$$
\hat{\mathfrak{h}}:=\operatorname{ker} \delta
$$

is a quantization of $\mathfrak{h}$.

This is the main result (Proposition 4) of [MR03] as it was reformulated in [BM11]. Furthermore, Meusburger proved some correspondences between $\hat{\mathfrak{h}}$ and $\mathfrak{h}$ : the subalgebra $\mathfrak{U}$ and the abelian subalgebra a have quantized analoga $\hat{\mathfrak{U}}$ and $\hat{a}$ respectively, and like $\mathfrak{h}$, its quantum analogon $\hat{\mathfrak{h}}$ is equipped with an action of $\mathfrak{s}(3, \mathbb{C})($ for $d=4)$, the parity operation defined by exchanging corresponding plusses and minusses and complex conjugation [Meu01, Section 6.4]. It was however demonstrated by G. Handrich, C. Paufler, J. B. Tausk and M. Walter that the quantum analogon $\hat{\mathfrak{h}}=\hat{\mathfrak{a}} \ltimes \hat{\mathfrak{U}}$ of conjecture 3.2.8 is false [HPTW02, p. 4].

Example 3.3.11. We construct a quantized lifting $\left[\hat{L}_{2}, \hat{L}_{1}\right] \in \hat{\mathfrak{h}}$ of the invariant $\left\{L_{2}, L_{1}\right\} \in \mathfrak{h}$. This is done in detail because it serves as part of a motivation example of the upcoming conjecture 3.4.2. We begin by constructing quantized liftings of the invariants $L_{1}$ and $L_{2}$, arranging products to be ascending with respect to the DegLex order with order of letters given by equation (1.67):

$$
\begin{aligned}
L_{1} & =-\sqrt{2} \mathcal{Z}_{0-++}^{(2)} \\
& =-\sqrt{2}(m e(-++)-e(0+) e(-+)) \\
& \mapsto-\sqrt{2}\left(m e(-++)-[e(0+), e(-+)]_{+}\right) \\
& =-\sqrt{2}\left(m e(-++)-e(0+) e(-+)+\frac{1}{2}[e(0+), e(-+)]\right) \\
& =-\sqrt{2}\left(m e(-++)-e(0+) e(-+)-\frac{\alpha}{2} h e(0+)\right)=: \hat{L}_{1} .
\end{aligned}
$$

Since the quantum corrections arise from reordering factors of the non-commutative product in $U\left(\mathfrak{g}_{h}\right)$, their value depends on the order on $\mathfrak{g}$ that is imposed on products. In the example, if the order of $e(0+)$ and $e(-+)$ is reversed, the quantum correction changes its sign. Analogously, we calculate (here, the commutator vanishes)

$$
\begin{aligned}
L_{2} & =\mathcal{Z}_{00++}^{(2)} \\
& =2 m e(0++)-e(0+) e(0+) \\
& \mapsto 2 m e(0++)-e(0+) e(0+)=: \hat{L}_{2} .
\end{aligned}
$$


We can now calculate the commutator

$$
\begin{aligned}
& {\left[\hat{L}_{2}, \hat{L}_{1}\right]=-\sqrt{2}([2 m e(0++), m e(-++)]+[2 m e(0++),-e(0+) e(-+)]} \\
& +\left[2 m e(0++),-\frac{\alpha}{2} h e(0+)\right]+[-e(0+) e(0+), m e(-++)] \\
& \left.+[-e(0+) e(0+),-e(0+) e(-+)]+\left[-e(0+) e(0+),-\frac{\alpha}{2} h e(0+)\right]\right) \\
& =-\sqrt{2}\left(-6 \alpha m^{2} e(0+++)-2 \alpha e(0+) e(0+) e(0+)\right. \\
& +5 \alpha m e(0+) \alpha e(0++)+\alpha m e(0++) e(0+)) \\
& =-\sqrt{2} \alpha\left(-6 m^{2} e(0+++)+6 m e(0+) e(0++)\right. \\
& -h[\underline{e(0+), e(0++)]}-2 e(0+) e(0+) e(0+)) \\
& =2 \sqrt{2} \alpha\left(3 m^{2} e(0+++)-3 m e(0+) e(0++)+e(0+) e(0+) e(0+)\right) \text {. }
\end{aligned}
$$

Note that the quantum correction is zero again because the underlined bracket vanishes.

\subsection{Leading terms of exceptional elements}

After the above review of the basic background of quantization, this section introduces some new results on the leading terms of some exceptional elements. As it was mentioned before, an exceptional element $L_{(\mathbb{1})}$ in the Poisson algebra of invariants $\mathfrak{h}$ cannot be generated from invariants $h, h^{\prime}$ of lower degree because if it could be generated that way, the leading term of lead $L_{(1)}$ could be written as a Lie bracket of leading terms of invariants $h$ and $h^{\prime}$, which leads to a contradiction [PR86, p. 622]. While the argument itself does not exclude the possibility that lead $L_{(1)}$ could be written as a Lie bracket of any two Euler-Lyndon elements, it leaves open the question whether this is possible. If the answer were no, then $g$ would not be finitely generated because then there would be an infinite number of elements lead $L_{(1)}$ that could not be rewritten as Lie brackets.

Surprisingly, $e(-000+)=$ lead $L_{3}$, was found in quantum corrections in the stratum $\hat{\mathfrak{h}}_{5}$ of the quantum algebra of invariants. A calculation indicating this was first performed by Meusburger in the course of her Diplomarbeit and later verified in the Diplomarbeit [Han09]. Since the quantum correction was calculated using the Pohlmeyer-Rehren Lie algebra $\mathfrak{g}$, this points towards a positive answer to the question given above.

Example 3.4.1. In order to understand the occurrence of lead $L_{3}$ better, we calculate the contributions of the quantum lifting $\left[\left[\hat{L}_{2}, \hat{L}_{1}\right],\left[\hat{L}_{-2}, \hat{L}_{-1}\right]\right]_{+}$of the invariant $\left\{L_{2}, L_{1}\right\}\left\{L_{-2}, L_{-1}\right\}$ to lead $L_{(3)}$. The term "contribution" to lead $L_{(3)}$ is a shorthand for the projection onto the subspace of $\hat{\mathfrak{h}}$ spanned by elements that have a factor of an Euler word with the same letter content as lead $L_{(3)}$, i.e. three zeroes and one plus and minus each. In example 3.3.11, we calculated

$$
\left[\hat{L}_{2}, \hat{L}_{1}\right]=2 \sqrt{2} \alpha\left(3 m^{2} e(0+++)-3 m e(0+) e(0++)+e(0+) e(0+) e(0+)\right) .
$$


Analogously, we calculate

$$
\left[\hat{L}_{-2}, \hat{L}_{-1}\right]=2 \sqrt{2} \alpha\left(3 m^{2} e(0---)-3 m e(0-) e(0--)+e(0-) e(0-) e(0-)\right) .
$$

The anticommutator of these terms can be rewritten using linearity as a sum of nine anticommutators of the individual summands.

- The anticommutator $72 \alpha^{2} m^{4}[e(0+++), e(0---)]_{+}$has Euler elements with two zeroes total, so its quantum correction cannot contribute to lead $L_{(3)}$.

- First note that the Euler words in the anticommutator $72 \alpha^{2} m^{3}[e(0+++), e(0-) e(0--)]_{+}$ contain only three zeroes in total. So only the $[\cdot, \cdot]_{ \pm}$part of the decomposition $[\cdot, \cdot]=[\cdot, \cdot]_{ \pm}+[\cdot, \cdot]_{0}$ of the bracket obtained from reordering the noncommutative factors can contribute to lead $L_{3}$ and will henceforth be considered because $[\cdot, \cdot]_{0}$ reduces the number of zeroes. Now, using Leibniz's rule on the bracket, we obtain

$$
\begin{aligned}
& 36 \alpha^{2} m^{3}[e(0+++), e(0-) e(0--)]_{ \pm} \\
= & 36 \alpha^{2} m^{3}\left([e(0+++), e(0-)]_{ \pm} e(0--)+e(0-)[e(0+++), e(0--)]_{ \pm}\right) .
\end{aligned}
$$

Observe that the bracket in the right hand summand contains only two zeroes and therefore cannot contribute to lead $L_{3}$. The remaining term is

$$
\begin{aligned}
& 36 \alpha^{2} m^{3}[e(0+++), e(0-)]_{ \pm} e(0--) \\
= & -36 \alpha^{3} m^{3} h e(00++) e(0--) \\
= & -36 \alpha^{3} m^{3} h(e(0--) e(00++)+[e(0--), e(00++)]) .
\end{aligned}
$$

The first summand is in ascending order again, and the quantum correction stems from the second summand. Because only three zeroes occur, its contribution to lead $L_{(3)}$ is contained in

$$
-36 \alpha^{3} m^{3} h[e(0--), e(00++)]_{ \pm}=36 \alpha^{3} m^{3} h^{2}(e(000-+)+e(000+-)+e(00+0-)) .
$$

- Analogously, the contribution of the quantum correction of the anticommutator $72 \alpha^{2} m^{3}[e(0+) e(0++), e(0---)]_{+}$to lead $L_{3}$ is

$$
36 \alpha^{3} m^{3} h^{2}(e(000-+)+e(000+-)+e(00-0+)) .
$$

- Applying Leibniz's rule to brackets arising in the reordering of the remaining six anticommutators produces Euler elements that cannot contribute to lead $L_{3} \in \mathfrak{g}_{3}$, because they are of degree $l \leq 2$.

We have shown that the sum of the contributions to lead $L_{(3)}$ from all anticommutators is

$$
36 \alpha^{3} m^{3} h^{2}(2 e(000-+)+2 e(000+-)+e(00+0-)+e(00-0+))=36 \alpha^{3} m^{3} h^{2} e(-000+),
$$

which actually is a multiple of lead $L_{3}$. 
A question suggesting itself in the context of the discovery of lead $L_{(3)}$ in a quantum correction is if leading terms of other exceptional elements can also be found in quantum corrections of elements of higher degree. Being the only known obstacle, this would be a necessary condition for finite generation of the quantum algebra of invariants $\hat{\mathfrak{h}}$.

While a general statement for all $L_{(\mathbb{D}}$ could not be reached, we do have some tentative results that lead to a general conjecture.

Conjecture 3.4.2. The quantum corrections of the following terms contain the leading term of the exceptional elements $L_{(1)}$ for all $l=2 n+3, n \in \mathbb{N}_{0}$ :

$$
\left\{\ldots\{\left\{L_{-2}, L_{-1}\right\}, \underbrace{\left.\left.L_{0}\right\}, \ldots, L_{0}\right\}}_{n} \cdot\left\{\ldots\{\left\{L_{2}, L_{1}\right\}, \underbrace{\left.\left.L_{0}\right\}, \ldots, L_{0}\right\}}_{n} \in \mathfrak{h}_{2 n+5} \text {. }\right.\right.
$$

This conjecture is supported by two observations:

1. The conjecture has been verified in Mathematica up to $l=9$. For higher $l$, the computation was not possible due to lack of RAM.

2. In the rest frame, the $L_{i}$ are sums of terms of the form me(abc) and $e(a b) e(c d)$, where $a, b, c, d \in X$ and by linearity, the product above can be expanded into summands that inherit only one of those terms from each $L_{i}$. The sum can then be developed into a linear combination of linearly independent vectors including lead $L_{(\mathbb{D}}$. Finding the coefficient of lead $L_{(\mathbb{D})}$ is a straightforward but laborious calculation (in essence a generalized version of example 3.4.1) described in appendix C. A closed term for the coefficient could be found, but being composed of a large number of summations, binomial coefficients, powers of -1 and Kronecker delta functions all with arguments depending on each other, it was so unwieldy that it resisted attempts to be proven to be nonzero in the case of arbitrary $l$.

We now leave the question of finding leading terms of exceptional elements in quantum corrections in $\hat{\mathfrak{b}}$ and conclude the chapter with a positive answer to the question stated at the beginning of this section if is possible to generate the leading term of each of the exceptional elements $L_{(1)}$ in $\mathfrak{g}$.

Proposition 3.4.3. The leading terms of the exceptional elements $L_{(1)}$ with $l=2 n+1$ and $n \in \mathbb{N}_{+}$ can be generated the following way:

$$
\text { lead } L_{(1)}=e\left(-0^{l+2}+\right)=-\frac{1}{2 l}\left[e(0-0+), e\left(-0^{l}+\right)\right]+z,
$$

where $z$ is a linear combination of Euler-Lyndon elements with 1 zeroes.

Proof. Because of the construction of the Lie algebras $[\cdot, \cdot]_{ \pm}$and $[\cdot, \cdot]_{0}$ in proposition 1.8.1.

$$
\left[e(0-0+), e\left(-0^{l}+\right)\right]=\left[e(0-0+), e\left(-0^{l}+\right)\right]_{ \pm}+\left[e(0-0+), e\left(-0^{l}+\right)\right]_{0} .
$$

We begin by noting that the latter summand, which we call $2 l z$, is a linear combination of Euler-Lyndon elements with $l$ zeroes because the Lie bracket $[\cdot, \cdot]_{0}$ diminishes the number of zeroes by two. 
Considering the fact that $l$ is odd, we calculate

$$
\begin{aligned}
& {\left[e(0-0+), e\left(-0^{l}+\right)\right]_{ \pm} } \\
= & -2 \cdot\left((-1)^{4+4+l+2} e\left(\left[\begin{array}{l}
0 \\
+0
\end{array} 0^{l}-\right)+(-1)^{4+4+1} e\left(0-0^{l+1}+\right)\right)\right. \\
= & 2 \cdot\left(e\left(0+0^{l+1}-\right)+2 e\left(+0^{l+2}-\right)+(-1)^{l+2+2-2} e\left(\begin{array}{c}
0 \\
+0^{l+1}-
\end{array}-\right)\right) \\
= & 2 \cdot\left(e\left(0+0^{l+1}-\right)+2 e\left(+0^{l+2}-\right)\right. \\
& \left.-e\left(0+0^{l+1}-\right)-(l+2) e\left(+0^{l+2}-\right)\right) \\
= & -2 l \cdot e\left(+0^{l+2}-\right) \\
= & -2 l \cdot e\left(-0^{l+2}+\right) .
\end{aligned}
$$

It should be noted that this result seems to point in the direction of finite generation of $\mathfrak{g}$, but it does not prove it because neither are the elements considered the only exceptional elements in $\mathfrak{h}$ nor is it clear if another infinite series of elements of $\mathfrak{g}$ exists the entries of which cannot be written as a Lie bracket of elements of $\mathfrak{g}$ of lower degree. 


\section{Chapter 4}

\section{Computational Applications of Hall-Bases in Non-Free Graded Lie Algebras}

\subsection{Motivation}

This chapter is motivated by two related problems that arise when studying the PohlmeyerRehren Lie algebra g: the search for generators and a systematic exploration of the relations between the multiple Lie brackets of those generators. We know the structure of $\mathfrak{g}$ and its strata $\mathfrak{g}_{l}$ as vector spaces and can calculate the Lie bracket on a basis of $\mathfrak{g}$ by using formula (1.54). We don't however have a way to calculate the relations between multiple Lie brackets of some set of possible generators directly, i.e. without reference to this basis. Regarding generators of $\mathfrak{g}$, we have the following conjectures ${ }^{1}$.

Conjecture 4.1.1. $\mathrm{g}$ is finitely generated.

More specifically, for $d \geq 3$ (in contrast to proposition 1.5.3)

Conjecture 4.1.2. The subalgebra $\mathfrak{g}_{\geq 1}$ of the Pohlmeyer-Rehren Lie algebra $\mathfrak{g}$ is generated by a basis of its first stratum, $\mathfrak{g}_{1}$.

Since the entirety of $\mathfrak{g}_{1}$ is the direct sum of its irreducible $\mathfrak{g}_{0}$-modules that in turn are the orbits of their respective highest weight vectors under the action of $\mathfrak{g}_{0}$ (a direct application of theorem 2.3.311), the following conjecture would imply 4.1.2 although the number of generators is lower:

Conjecture 4.1.3. $\mathfrak{g}_{\geq 0}$ is generated by generators of $\mathfrak{g}_{0}$ and the highest weight vectors in $\mathfrak{g}_{1}$.

Computer algebra can be a useful tool insofar as it can be used to falsify specific candidates for sets of generators. Since the number of elements of $\mathfrak{g}$ that can be checked by direct computation for linear dependence from multiple brackets is necessarily finite (and this set of elements trivially generates itself), we cannot hope to falsify conjecture 4.1.1 this way. It could, however, be possible to find counterexamples to conjectures 4.1 .2 and 4.1 .3 this way.

\footnotetext{
${ }^{1}$ Note that the restriction to $\mathfrak{g}_{\geq 1}$ or $\mathfrak{g}_{\geq 0}$ is mostly cosmetic as the stratum $\mathfrak{g}_{-1}$ is central.
} 


\subsection{The Philip Hall algorithm for free Lie algebras}

A naive way to approach the problems lined out above is to consider all possible multiple brackets of elements of the set of generators $X$. We can identify them with elements of the free magma $M(X)$. Now we can catalogue all the relations between elements of $M(X)$, which is a basis of the free algebra $A(X)$. This is quite easy to implement on a computer, but it is inefficient because it does not take the Lie structure of $\mathfrak{g}$ into account, as will be explained in more detail at the beginning of the next section. So let us consider the Lie structure. In order to establish the notation that will be used here, let us review some basic definitions and facts. The following definitions are taken from [dG00, chapter 7], which this section largely follows except for the applications to the Pohlmeyer-Rehren Lie algebra.

\subsubsection{Basic definitions}

Definition 4.2.1 (free magma). Let $X$ be a set. Then the set $M(X)$ defined inductively by

1. $X \subset M(X)$,

2. if $m, n \in M(X)$, then also the ordered pair $(m, n) \in M(X)$

is called the free magma over $X$. Elements of $M(X)$ are called monomials.

The map $\cdot: M(X) \times M(X) \rightarrow M(X), m \cdot n:=(m, n)$ is known as the free (non-associative and (if $\# X>1)$ non-commutative) binary operation.

We also define the degree $\operatorname{deg}_{\mathrm{H}}$ by $\operatorname{deg}_{\mathrm{H}} x=1$ for all $x \in X$ and $\operatorname{deg}_{\mathrm{H}}(m, n)=\operatorname{deg}_{\mathrm{H}} m+\operatorname{deg}_{\mathrm{H}} n$. $\operatorname{deg}_{\mathrm{H}}$ is a gradation on $M(X)$, and in order to distinguish it from other gradations that will be introduced later, we call it the monomial degree.

Definition 4.2.2 (free algebra). Let $\mathbb{K}$ be a field. Then define $A(X)$ as the vector space spanned over $\mathbb{K}$ by $M(X)$. Extending the operation - from $M(X)$ bilinearly to $A(X)$ equips $A(X)$ with the structure of a (non-associative) algebra; $A(X)$ is called the free algebra over $X$.

The degree is extended to $A(X)$ by considering each element of $A(X)$ as a linear combination of monomials; the degree of an element of $A(X)$ is the maximum of the degrees of the monomials that occur with nonzero coefficient.

Definition 4.2.3 (free Lie algebra). Let $I_{\text {Lie }}$ be the ideal of $A(X)$ generated by the elements

$$
(m, m), \quad(m,(n, p))+(n,(p, m))+(p,(m, n))
$$

for $m, n, p \in M(X)$. Then $L(X):=A(X) / I_{\text {Lie }}$ is a Lie algebra, called the free Lie algebra over $X$.

Note that the inclusions $\iota_{M}, \iota_{A}$ and projection $\pi$ give rise to the canonical map

$$
X \stackrel{\iota_{M}}{\hookrightarrow} M(X) \stackrel{\iota_{A}}{\hookrightarrow} A(X) \stackrel{\pi}{\rightarrow} L(X)
$$

that allows us to identify elements of $X, M(X), A(X)$ with elements of $L(X)$ as a shorthand.

The following basic theorem shows that any free Lie algebra can be understood as a quotient of a free Lie algebra ${ }^{2}$

\footnotetext{
${ }^{2}$ But not as a subalgebra, since every subalgebra of a free Lie algebra is free itself by the Shirshov-Witt theorem.
} 
Theorem 4.2.4. Let $\mathrm{G}$ be a Lie algebra generated by a subset $X \subset \mathrm{G}$. Then

1. there is a surjective morphism $\phi: L(X) \rightarrow \mathrm{G}$ and

2. $\mathrm{G} \cong L(X) / I$ with the ideal $I=\operatorname{ker} \phi$.

Proof. See [dG00, Proposition 7.1.5 f.].

Remark 4.2.5. If all relations in $I$ are homogeneous with respect to the monomial degree, then $L(X) / I$ is graded by monomial degree as well. Due to theorem 1.2 .24 , this applies to the Pohlmeyer-Rehren Lie algebra $\mathfrak{g}$.

The above theorem 4.2 .4 tells us that we can learn more about $\mathrm{G}$ by studying $I$, which a large part of the remainder of this chapter will be concerned with. But before we do that, let us spend the rest of this section reviewing information about the free Lie algebra $L(X)$.

\subsubsection{Hall sets}

By construction of the free algebra, $M(X)$ is a (K-linear) basis of $A(X)$. Since $L(X)=A(X) / I_{\text {Lie, }}$, $M(X)$ spans $L(X)$ as well, but due to the relations introduced by the ideal $I_{\text {Lie, }}$, the set $M(X)$ is not linearly independent in $L(X)$.

Fortunately, the concept of Hall sets provides us with a family of subsets of $\pi(M(X))$ each of which is linearly independent but still spans $L(X)$.

Definition 4.2.6 (Hall set, Hall order). Let $X$ be a set and let $<$ be a total order on $M(X)$. We define the set $H \subset M(X)$ by

1. $X \subset H$,

2. if $h_{1}, h_{2} \in M(X)$ then $\left(h_{1}, h_{2}\right) \in H$ iff $h_{1}, h_{2} \in H$ and

(a) $h_{1}<h_{2}$

(b) $h_{2} \in X$ or $h_{2}=(a, b)$ with $a \leq h_{1}$.

If the order $<$ satisfies $\left(h_{1}, h_{2}\right)>h_{2}$ for all $h_{1}, h_{2} \in H$ such that $\left(h_{1}, h_{2}\right) \in H$, the order $<$ is called a Hall order and $H$ (which is uniquely determined by $<$ ) is called the Hall set corresponding to $<3$

In chapter 1 we had introduced the concept of the word algebra, which is just another term for the free associative (but non-commutative) algebra $\mathbb{K}\langle X\rangle$ over an alphabet $X$, spanned by the set of words $X^{*}$. It is not very surprising that there is a canonical connection ${ }^{4}$ to the concept of the free non-associative algebra $A(X)$ over the same alphabet spanned by the set of monomials $M(X)$.

\footnotetext{
${ }^{3}$ Note that the order $>$ is inverted compared to the notation in dG00, but consistent with the notation in [Ser65 p. 22]. This is done to maintain notational consistency with the degree of the Pohlmeyer-Rehren Lie algebra as well as the more intuitive convention of $\left(h_{1}, h_{2}\right)>h_{2}$ instead of $\left(h_{1}, h_{2}\right)<h_{2}$ as a condition for a Hall order. Several conventions for Hall sets that switch $h_{1}$ and $h_{2}$ or $a$ and $b$ exist, and due to the anticommutativity of the Lie bracket, they are all equivalent in the sense that theorem 4.2 .12 holds.

${ }^{4}$ Equivalently to the following definition (and similar to the definition of the free Lie algebra 4.2.3, the free associative algebra $\mathbb{K}\langle X\rangle$ can be defined as the quotient algebra $A(X) / I$ where $I$ is the ideal generated by elements of the form $(a,(b, c))-((a, b), c)$ for $a, b, c \in M(X)$, and the extension of $\phi$ as the canonical projection.
} 
Definition 4.2.7 (foliage, Hall word). Consider the map $\varphi: M(X) \rightarrow X^{*} \subset \mathbb{K}\langle X\rangle$ called foliage defined by

$$
\varphi(x):=\left\{\begin{array}{ll}
x & \text { if } x \in X \\
\varphi\left(x^{\prime}\right) \frown \varphi\left(x^{\prime \prime}\right) & \text { if } x=\left(x^{\prime}, x^{\prime \prime}\right) \in M(X) \backslash X
\end{array} .\right.
$$

Simply put, the foliage removes the non-associative order in which monomials are composed, turning them into (associative) words.

If $H$ is a Hall set relative to $<_{H}$ and $h \in H$, then $\varphi(h)$ is called a Hall word (relative to $<_{H}$ ). The foliage $\varphi$ can be linearly extended to a map $A(X) \rightarrow \mathbb{K}\langle X\rangle$.

We continue our general discussion of Hall sets with three basic examples of Hall orders.

Example 4.2.8 (some Hall orders). Let $m, n \in M(X)$.

1. The foliage $\varphi$ can be used to extend any order defined on $X^{*}$ to $M(X)$. Some of them are Hall orders. It is straightforward to show (cf. [dG00, lemma 7.8.4]) that the lexicographic order, defined by

$$
m<_{\text {Lex }} n: \Leftrightarrow \varphi(m)<_{\text {Lex }} \varphi(n)
$$

is a Hall order.

2. Analogously the graded lexicographic order is extended to $M(X)$ :

$$
m<\operatorname{DegLex} n: \Leftrightarrow \varphi(m)<_{\operatorname{DegLex}} \varphi(n) .
$$

3. $<$ DegLex is a special case of the following fact that is easy to demonstrate as well: Any order $>$ on $M(X)$ that satisfies

$$
\operatorname{deg}_{\mathrm{H}} m>\operatorname{deg}_{\mathrm{H}} n \Rightarrow m>n
$$

for all $m, n$ in $M(X)$ is a Hall order.

It is a useful fact that the set of Lyndon words, introduced in section 1.3, can be considered a particular Hall set.

Theorem 4.2.9 (Lyndon words as a Hall basis). The Hall words relative to $<_{\text {Lex }}$ are exactly the Lyndon words. $\left.\varphi\right|_{H}$ is injective.

Proof. See [dG00, Chapter 7]. De GraAf defines $\varphi(H)$ as the set of Lyndon words and proves lemma 7.8.5, which states that they can be characterized by the property used to define them in definition 1.3.2. He also gives an algorithm to compute the corresponding Hall element to a Hall word.

Since $\left.\varphi\right|_{H}$ is a bijection between elements of the Hall set and Hall words, we will use the terms synonymously, as the literature also frequently does. Its inverse $\left.\varphi\right|_{H} ^{-1}$ is called bracketing. 
Remark 4.2.10. The fact that $\left.\varphi\right|_{H}$ is bijective gives rise to an interesting question for the Pohlmeyer-Rehren Lie algebra: If the Euler-Lyndon words are Hall words, can we find generators $x_{1}, \ldots, x_{d} \in \mathfrak{g}$ such that the Lyndon word $\left(y_{1} \ldots y_{n}\right)$ in any Euler-Lyndon word $e\left(y_{1} \ldots y_{n}\right)$ corresponds to a way to generate that Euler-Lyndon word in the following way:

$$
e\left(y_{1} \ldots y_{n}\right)=\left.z \varphi\right|_{H} ^{-1}\left(x_{y_{1}} \ldots x_{y_{n}}\right) \text { with } z \in \mathbb{C} ?
$$

The right hand side of this equation is a multiple Lie bracket of the elements $x_{y_{1}} \ldots x_{y_{n}}$ where the order is controlled by the property that it is a Hall word.

We begin exploring this question for $d=3$. We also postulate that $x_{i}$ are compatible with the magnetic quantum numbers in the following way: $x_{+} \in \mathfrak{g}^{1}, x_{0} \in \mathfrak{g}^{0}, x_{-} \in \mathfrak{g}^{-1}$. If this is the case, the magnetic quantum numbers on both sides of equation (4.6) automatically match due to additivity of the magnetic quantum numbers (lemma 1.6.4). Otherwise, for the same reason, the multiple Lie brackets on the right hand side have summands with magnetic quantum numbers different from the left hand side that all have to vanish for every Hall word on the left hand side.

Note that for $n=2$, satisfying equation (4.6) is possible with

$$
x_{0}=e(-+), \quad x_{ \pm}=e(0 \pm)
$$

(a calculation that also shows that for $d=3, \mathfrak{g}_{0}$ is isomorphic to $\mathfrak{s l}_{2}$ by giving us the structure constants).

However, for $n=1$; the $x_{i}$ are fixed by equation (4.6) to be (up to complex constants)

$$
x_{0}=e(0), \quad x_{ \pm}=e( \pm),
$$

but these elements do not satisfy equation (4.6) for any $n>1$ because they are in the center of $\mathfrak{g}$ so the right hand side would be zero. So, the initial question can be answered in the negative.

But what if we exclude the central subalgebra $\mathrm{g}^{-1}$, in other words also presuppose $n \geq 2$ ? In general, we can not satisfy equation (4.6) for all $n$ with homogeneous $x_{i}$ (with respect to the usual gradation deg on $\mathfrak{g}$, cf. thm. 1.4.24) because then

$$
\operatorname{deg} x_{0}+2 \operatorname{deg} x_{+}=\operatorname{deg} e(0++)=1=\operatorname{deg} e(00+)=2 \operatorname{deg} x_{0}+\operatorname{deg} x_{+}
$$

which is impossible to satisfy with $x_{i} \in \mathbb{N}_{0}$. But as the following proposition shows, even inhomogeneous $x_{i}$ cannot satisfy equation (4.6) for all $n \geq 2$.

Proposition 4.2.11. There are no $x_{0} \in \mathfrak{g}^{0}, x_{ \pm} \in \mathfrak{g}^{ \pm 1}$ such that equation (4.6) holds for all $n \geq 2$.

Proof. We write $x_{0}, x_{+}$in terms of the basis of $\mathfrak{g}_{0}$ and $\mathfrak{g}_{1}$ lined out in subsection 2.3.3

$$
\begin{aligned}
& x_{+}=a e+b^{1} v^{1}+c^{2} v^{1}+u, \\
& x_{0}=i 2 h+j^{1} v^{0}+k^{2} v^{0}+w
\end{aligned}
$$

with coeffcients $a, b, c, i, j, k \in \mathbb{C}$ and remaining terms $u, w \in \mathfrak{g}_{\geq 2}$. Postulate 4.6 implies the equations (the complex coefficients $z$ can be absorbed into the $a, b, c, i, j, k$ )

$$
\begin{aligned}
{\left[x_{0}, x_{+}\right] } & =e(0+), \\
{\left[x_{+},\left[x_{0}, x_{+}\right]\right] } & =e(0++) .
\end{aligned}
$$


Substituting the values for $x_{0}$ and $x_{+}$from equation (4.9) and comparing the coefficients of the Euler-Lyndon basis for degrees 0 and 1 (noting that all terms with $u$ or $v$ are of degree $\geq$ 2 ), we obtain a system of equations which has the unique solution

$$
a=i=1 \text { and } b=c=j=k=0 .
$$

Analogously, from equation (4.10), we can deduce

$$
a k^{2}=1,
$$

in contradiction to the solution 4.11 of equation (4.9).

Now that we have established Hall sets, it is time to introduce a very important application of Hall bases that is eminently useful in the theory of free Lie algebras and also is the foundation of the entire chapter.

Theorem 4.2.12. Let $H$ be a Hall set in $M(X)$. Then $H$ is a (K-linear) basis of the free Lie algebra $L(X)$.

Proof. This is [dG00, Corollary 7.7.7].

In this situation, we refer to $H$ as a Hall basis of $L(X)$. An intuitive way to think about the Hall set in light of this theorem is that condition 2a (for the Hall set, definition 4.2.6) eliminates elements of $M(X)$ that are linerarly dependent because of the alternativity of the Lie bracket and condition $2 \mathrm{~b}$ eliminates elements of $M(X)$ that are linearly dependent because of its Jacobi relation.

Before an algorithm providing a way to list the elements of a Hall set for practical computations is given, we apply the previous two theorems to prove a classical result from the theory of free Lie algebras originally proved by WITT in 1937 [Wit37][p. 152f]. It allows us to calculate the dimension of the strata (with respect to the monomial degree) of the free Lie algebra in terms of theorem 1.3.6

Corollary 4.2.13 (WITT's formula). Let X be a finite set. Then

$$
\operatorname{dim} L(X)_{l}=\operatorname{NumLyndon}(l, \# X)=\frac{1}{l} \sum_{q \mid l} \mu(q)(\# X)^{l / q} .
$$

Proof. The dimension of $L(X)_{l}$ is independent of the choice of basis. The Hall set relative to $<_{\text {lex }}$ is a basis by theorem 4.2.12, and by theorem 4.2.9 the Hall words of degree $l$ can be identified with the Lyndon words of length $l$, the number of which is given by NumLyndon $(l, \# X)$.

\subsubsection{HALL's algorithms}

In order to actually use theorem 4.2.12 in practical applications, it is very helpful to be able to list the elements of a Hall set. The following two algorithms accomplish this, up to a given degree. In the sequel, we will impose the condition that $X$ be a finite set so we can represent it in a computer as a list of elements.

The definition of the Hall set (see 4.2.6) gives rise to the algorithm IsHallElement that allows us to compute whether any given monomial $h \in M(X)$ is a Hall element by iteratively considering all monomials $h$ is composed of. The input is $h \in M(X)$, the output is the (boolean) truth value of $h \in H$ where $H$ is the Hall set determined by the Hall order $<$. 

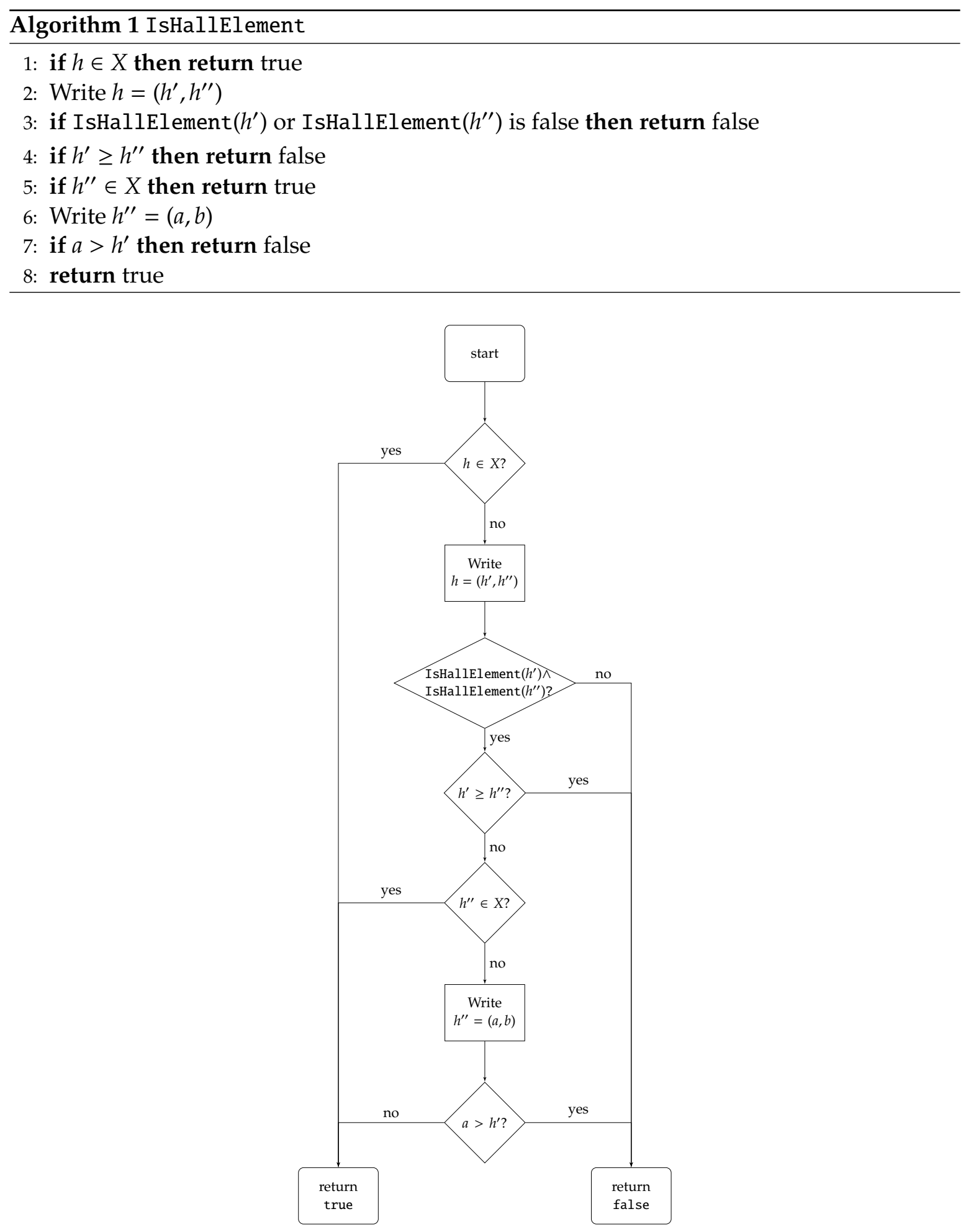

Figure 4.1: Flow chart of algorithm IsHallElement

Note that the algorithm IsHallElement can determine if a monomial is contained in $H$ without prior knowledge of $H$. But we can use it as part of the algorithm Hall to find all 
elements of a Hall set up to a given degree. Also note that free Lie algebras have infinite dimension, so Hall sets are infinite (except in the case of the free Lie algebra generated by one generator, which is abelian and therefore one-dimensional), and this is the best that can be expected from an algorithm that terminates.

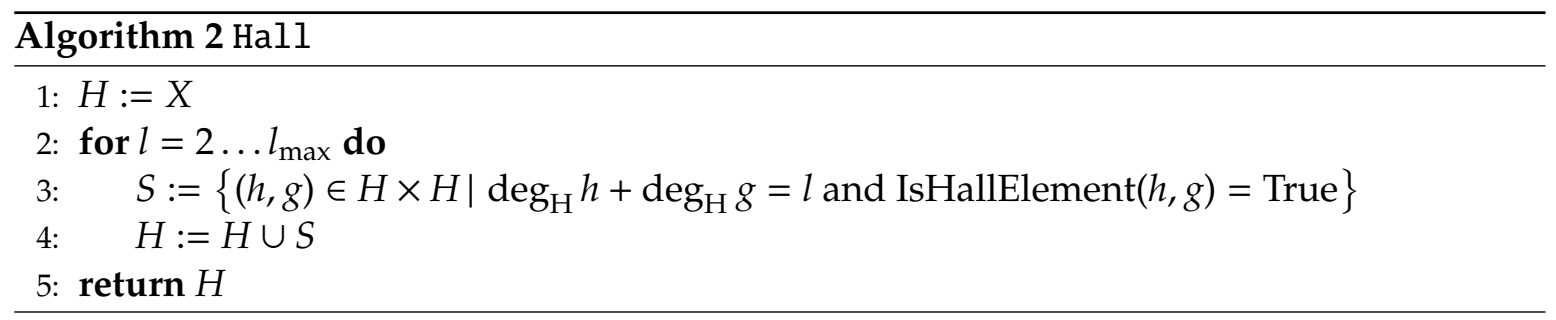

The input of this algorithm is the finite set $X$, the Hall order $<$ on $M(X)$ and a natural number $l_{\max }>1$. The output is the set

$$
\mathrm{H}=\left\{h \in H \mid \operatorname{deg}_{\mathrm{H}} h \leq l_{\max }\right\} .
$$

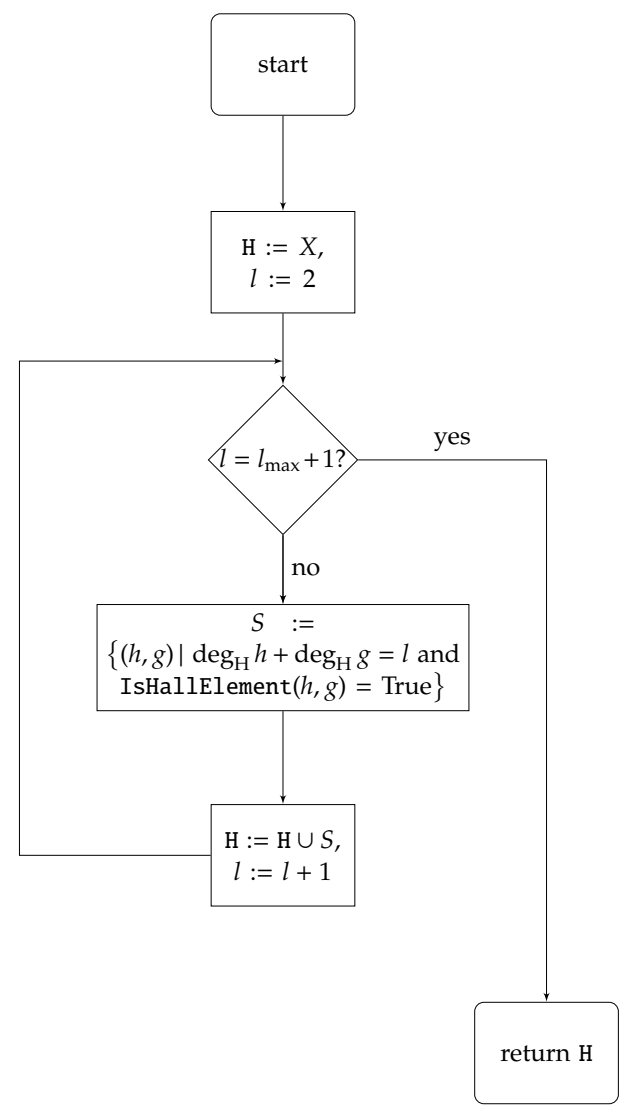

Figure 4.2: Flow chart of algorithm Hall

Summing up the key notions of this section, the algorithm Hall allows us to compute all elements up to a given degree $l_{\max }$ of the Hall set $H$ that is basis of the free Lie algebra $L(X)$ generated by a finite set $X$ relative to a given Hall order $<$. 
Example 4.2.14. We compute the elements of degree $l_{\max } \leq 5$ of the free Lie algebra generated by the elements $L_{1}, L_{2}$ using Hall order $<_{H}=$ DegLex. Note that the algorithm does not specify an order in which the elements $s \in S$ are computed.

\begin{tabular}{|c|c|}
\hline$l$ & $s \in S$ \\
\hline 2 & {$\left[L_{1}, L_{2}\right]$} \\
\hline 3 & {$\left[L_{1},\left[L_{1}, L_{2}\right]\right]$} \\
3 & {$\left[L_{2},\left[L_{1}, L_{2}\right]\right]$} \\
\hline 4 & {$\left[L_{1},\left[L_{1},\left[L_{1}, L_{2}\right]\right]\right]$} \\
4 & {$\left[L_{2},\left[L_{1},\left[L_{1}, L_{2}\right]\right]\right]$} \\
4 & {$\left[L_{2},\left[L_{2},\left[L_{1}, L_{2}\right]\right]\right]$} \\
\hline 5 & {$\left[L_{1},\left[L_{1},\left[L_{1},\left[L_{1}, L_{2}\right]\right]\right]\right]$} \\
5 & {$\left[L_{2},\left[L_{1},\left[L_{1},\left[L_{1}, L_{2}\right]\right]\right]\right]$} \\
5 & {$\left[L_{2},\left[L_{2},\left[L_{1},\left[L_{1}, L_{2}\right]\right]\right]\right]$} \\
5 & {$\left[L_{2},\left[L_{2},\left[L_{2},\left[L_{1}, L_{2}\right]\right]\right]\right]$} \\
5 & {$\left[\left[L_{1}, L_{2}\right],\left[L_{1},\left[L_{1}, L_{2}\right]\right]\right]$} \\
5 & {$\left[\left[L_{1}, L_{2}\right],\left[L_{2},\left[L_{1}, L_{2}\right]\right]\right]$} \\
\hline
\end{tabular}

\subsection{Extension to non-free Lie algebras}

Throughout this section, let $\mathrm{G}$ be a (not necessarily free) Lie algebra generated by a finite, linearly independent set $X \subset G$ and let $<_{H}$ be a Hall order on $M(X)$.

Theorem 4.2.4 told us that there is an ideal $I$ such that $G \cong L(X) / I$. After the brief discussion of $L(X)$ given above, let us discuss the ideal $I$. The underlying idea of this chapter is to find all relations between multiple Lie brackets of generators which were identified with $M(X)$, a basis of $A(X)$. So if we can compute, recursively by degree, all the terms of the form

$$
\phi \circ \pi(x) \in \mathrm{G} \cong L(X) / I, x \in M(X)
$$

and find all the relations between them, these relations will generate the ideal $\pi^{-1}(I)$, a subideal of which is $I_{\text {Lie }}$ (because $\pi^{-1}(0 \in I)=I_{\text {Lie }}$ ). If, as in the motivating case of the Pohlmeyer-Rehren Lie algebra, the structure of $G$ as a vector space is known, this is very easy to do in practice because the images under $\phi \circ \pi$ of the monomials are just all the different multiple Lie brackets of the generators. Since the number of monomials of a given monomial degree grows exponentially with monomial degree,

$$
\#\left\{x \in M(X) \mid \operatorname{deg}_{\mathrm{H}} x=l\right\}=(\# X)^{l},
$$

it is not very practical to do this by hand. However if we do this computation naively, i.e. without being able to recognize them (for instance using computer algebra systems), we will keep finding the elements of $I_{\mathrm{Lie}}$, the minimal relations of a Lie algebra, over and over again. Since we know both the number elements of $M(X)$ and the dimension of the free Lie algebra $L(X)$ (which is the largest Lie algebra generated by $X$ ) within the $l$-th stratum, a lower bound of the number of these relations in the $l$-th stratum is given by

$$
(\# X)^{l}-\operatorname{NumLyndon}(l, \# X) \text {. }
$$


This can be a significant number in practical computations, and it is helpful to suppress the elements of $I_{\text {Lie }}$ in our computations. This can be done by computing the relations between images of the elements of a Hall set over $X$ instead of the images of all the elements of $M(X)$.

Modifying the algorithm generating a Hall set given in the last section the following way achieves this: as opposed to the free case, the set $S$ is not linearly independent, but we can use it to iteratively extend to a basis of the stratum $\mathrm{G}_{l}$. In order to do this deterministically, we presuppose a total order $<_{s}$, called sorting order on $M(X)$ and treat $H$ and $B$ as tuples instead of sets (sometimes, we will bend the notation and use $\in$ to refer to entries of tuples). Thus we arrive at the following algorithm:

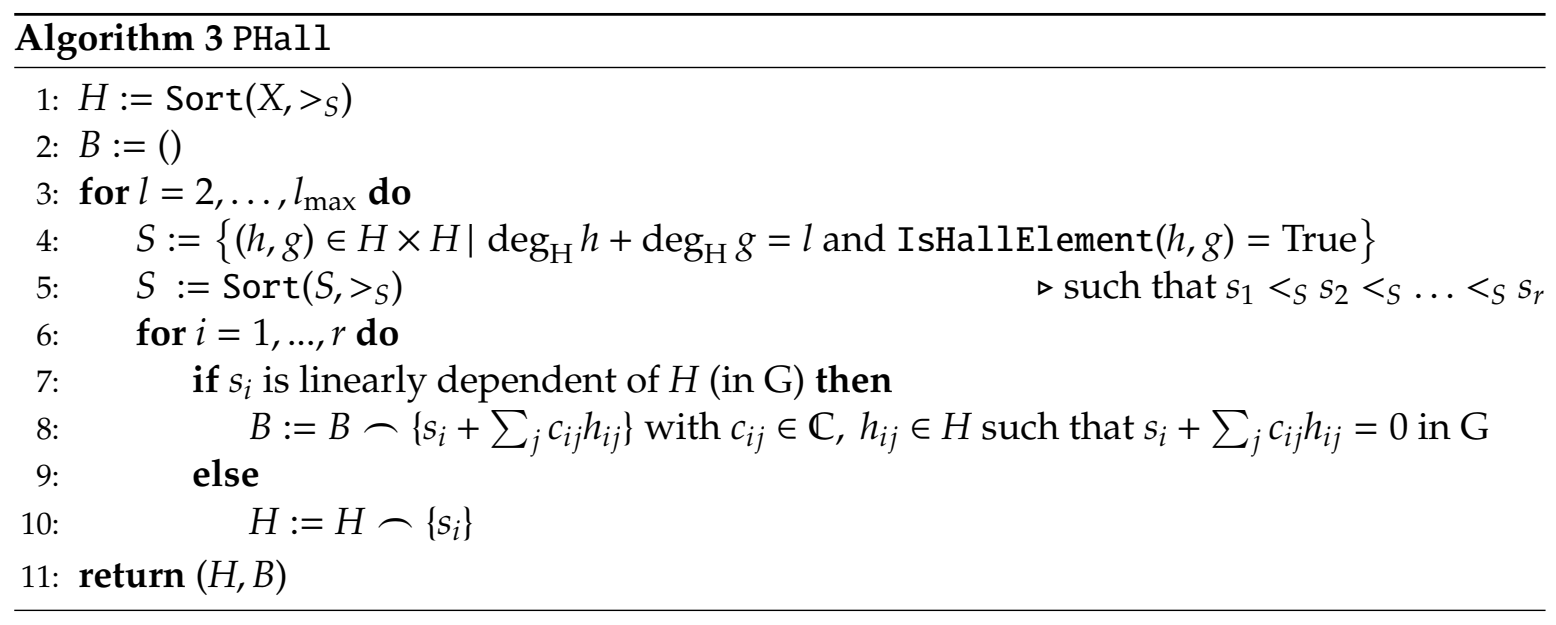

The input is a set $X$ generating $G$ and a maximal monomial degree $l_{\max }$ to compute up to. The outputs are ordered tuples $H$ and $B$. To prepare for later generalizations, we will speak of a gradation deg on $\mathrm{G}$ instead of the more specific $\operatorname{deg}_{\mathrm{H}}$ which is used in PHall. Define the subtuples of $H$ and $B$

$$
\begin{aligned}
H_{l} & :=\left(h_{i} \in H \mid \operatorname{deg} h=l\right)_{i}, \\
\bar{H}_{l} & :=\left(h_{i} \in H \mid \operatorname{deg} h \leq l\right)_{i}, \\
B_{l} & :=\left(b_{i} \in B \mid \operatorname{deg} b=l\right)_{i}, \\
\bar{B}_{l} & :=\left(b_{i} \in B \mid \operatorname{deg} b \leq l\right)_{i} .
\end{aligned}
$$

Note that the construction of the algorithm implies that $H_{k}$ and $B_{k}$ consist exactly of those elements that are added to $H$ and $B$ respectively during the iteration of the for loop where $l=k$. Furthermore, for PHall $H_{1}=X$, sorted by $<_{S}$, and $B_{1}=B_{0}=H_{0}=()$.

To be able to formulate some key properties of this algorithm, we give two connected definitions.

Definition 4.3.1 (leading monomial). ${ }^{5}$ Let $g \in A(X)$ and $<$ a total order on $M(X)$. Then the leading monomial (or leading term) $\mathrm{LM}(g)$ is the largest (with respect to $<$ ) monomial occurring in $g$ with a nonzero coefficient.

Definition 4.3.2 (self-reducedness). ${ }^{6}$ A set $S \subset A(X)$ is called self-reduced if $\operatorname{LM}(g)$ is no factor of $\operatorname{LM}(h)$ for $g, h \in S$ with $g \neq h$.

\footnotetext{
${ }^{5}$ cf. dG00 p. 261]

${ }^{6} \mathrm{cf}$.dG00 7.3 .4 (p. 262 f.)]
} 


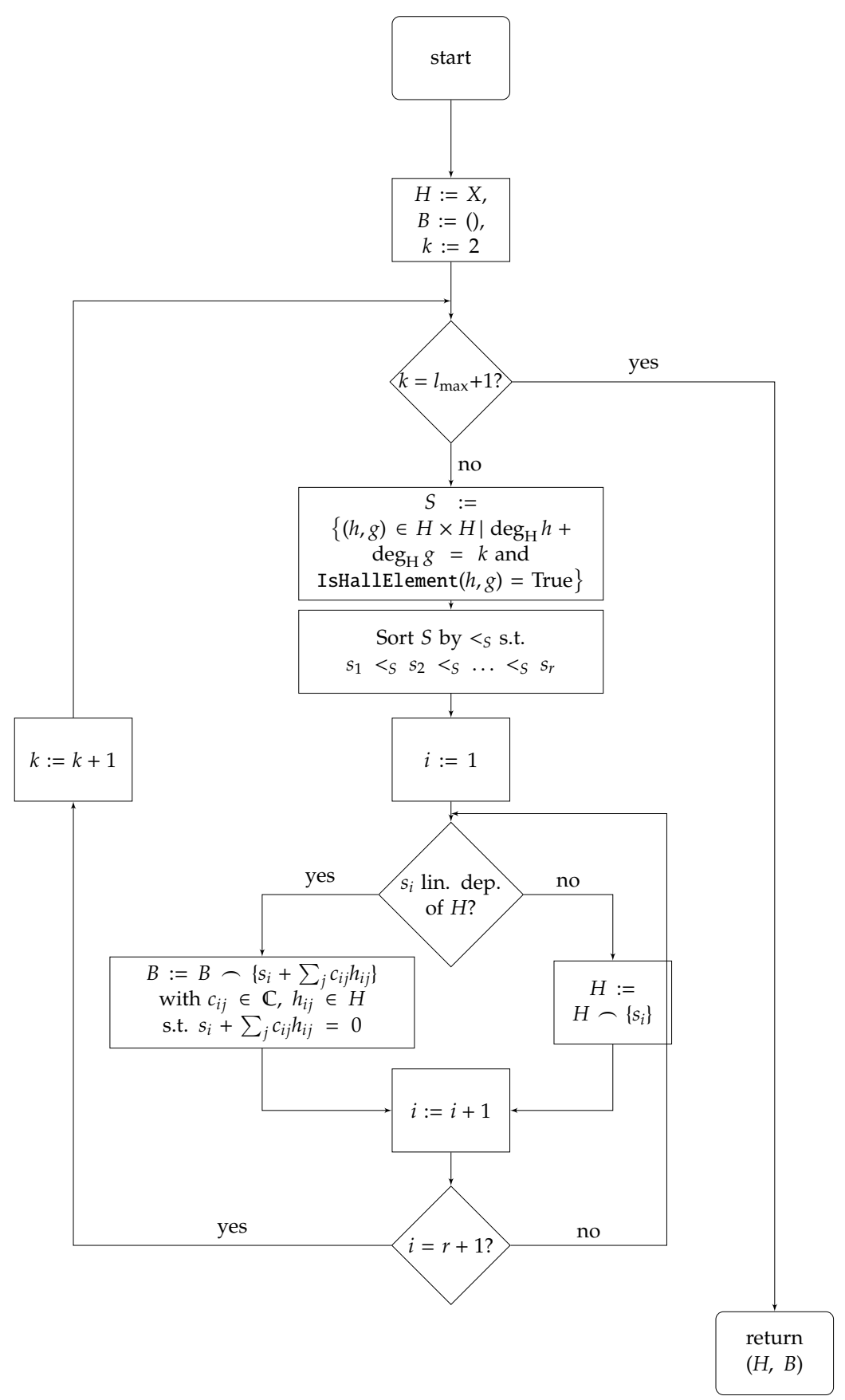

Figure 4.3: Flow chart of algorithm PHall 
Theorem 4.3.3 (properties of PHall). Designate $]^{7}$ the ideals of $L(X)$ generated by $B_{l}$ as $I_{l}$, as well as the ideal generated by $\bar{B}_{l}$ as $\bar{I}_{l}$ and define the vector spaces

$$
L_{l}:=\left\{x \in L(X) / \bar{I}_{l} \mid \operatorname{deg} x=l\right\}, \quad \bar{L}_{l}:=\bigoplus_{i=0}^{l} L_{i} .
$$

and

$$
\overline{\mathrm{G}}_{l}:=\bigoplus_{i=0}^{l} \mathrm{G}_{i}
$$

Then:

1. We have the following commutative diagram of linear maps (in fact the maps $\varphi_{l}$ are epimorphisms of Lie algebras):

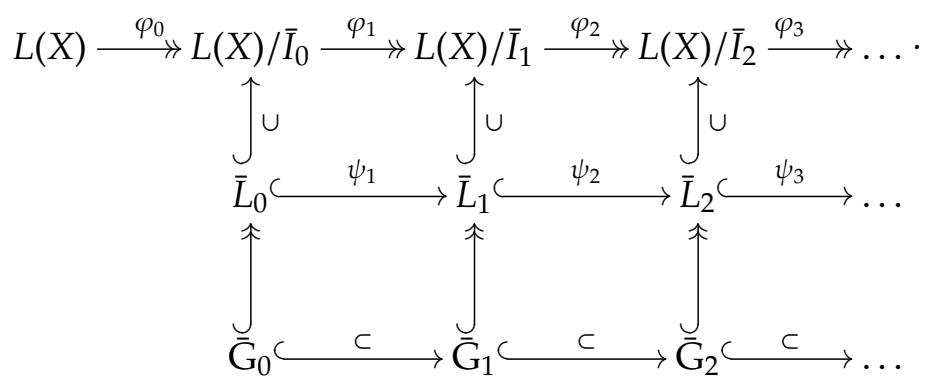

Use the notation $\bar{\varphi}_{l}:=\varphi_{l} \circ \varphi_{l-1} \circ \ldots \circ \varphi_{1} \circ \varphi_{0}$.

2. $H_{l}$ and $B_{l}$ are linearly independent tuples of elements of $L(X)$.

3. $\bar{\varphi}_{l} H_{l}$ is a basis of $L_{l}$,

4. $B$ is self-reduced (in the sense of 4.3.2).

5. Both $H$ and $B$ are in nondescenting order with respect to deg. $H_{l}$ and $B_{l}$ are in ascending order with respect to $<_{S}$. In particular, if $<_{S}$ satisfies

$$
\operatorname{deg} m>\operatorname{deg} n \Rightarrow m>_{S} n,
$$

then $H$ and $B$ are in ascending order with respect to $<_{S}$ as well.

6. The algorithm terminates.

Proof. 1. $\varphi_{0}$ is the canonical projection. For all $l \in \mathbb{N}_{+}$, because $I_{l+1}=\bar{I}_{l+1} / \bar{I}_{l}$, there is equality in the middle of

$$
L(X) / \bar{I}_{l} \stackrel{\pi_{l}}{\rightarrow}\left(L(X) / \bar{I}_{l}\right) / I_{l+1}=\left(L(X) / \bar{I}_{l}\right) /\left(\bar{I}_{l+1} / \bar{I}_{l}\right) \stackrel{v_{l}}{\hookrightarrow} L(X) / \bar{I}_{l+1}
$$

\footnotetext{
${ }^{7}$ Note that for PHall, as opposed to variations of this algorithm to be introduced later, $B_{0}=\{\}$, which implies $I_{0}=0, \bar{L}=0$ and $\bar{G}_{0}=0$, so the first column of the commutative diagram is trivial.
} 
where $\pi_{l}$ are the canonical projections and $v_{l}$ are the natural isomorphisms arising from the second isomorphism theorem for Lie algebras; their composition $\varphi_{l}:=v_{l} \circ \pi_{l}$ is the required epimorphism of Lie algebras.

Since $\operatorname{deg} I_{l+1} \geq \operatorname{deg} B_{l+1}=l+1$ and $\operatorname{deg} \bar{L}_{l} \leq l$, we conclude that the canonical projection $\bar{\pi}_{l}: \bar{L}_{l} \rightarrow \bar{L}_{l} / I_{l+1}$ is actually an isomorphism, and for all $l \in \mathbb{N}_{+}$set $\psi_{l}:=\bar{\iota}_{l} \circ \bar{\pi}_{l}^{-1}$ to be the composition of their inverses and the inclusions $\bar{l}_{l}:\left\{x \in L(X) / \bar{I}_{l+1} \mid \operatorname{deg} x \leq l\right\} \hookrightarrow \bar{L}_{l+1}$. By this construction, $\psi_{l}=\left.\varphi_{l}\right|_{\bar{L}_{l}}$ (except for different codomains) for all $l \in \mathbb{N}_{+}$, so the upper squares of the diagram commute.

The algorithm maps the basis elements of $\overline{\mathrm{G}}_{l}$ to all the basis elements of $\bar{L}_{l}$ (achieving this under different preconditions is the reason several variants of PHall will be introduced in the sequel), so the maps $\tau_{l}$ are isomorphisms of vector spaces for all $l \in \mathbb{N}_{0}$, and the lower squares of the diagrams commute because the degree is conserved.

2. This follows from the fact that in each step, $H_{l}$ and $B_{l}$ are disjunct subtuples of $S$, and $S$ is a basis of $\{g \in L(X) \mid \operatorname{deg} g=l\}$.

3. Follows from that fact that the elements of $H$ are selected by the if condition in step 7 so that their images under $\bar{\varphi}_{l}$ are linearly independent.

4. Let $b \in B$. This implies $\operatorname{LM}(b)=s_{i}$ in some iteration of the loop over $l$. If we factor $s_{i}=[h, g]$, then $h, g \in H$. But leading monomials of elements of $B$ are not contained in $H$.

5. Sorting by deg follows from the fact that the algorithm uses the degree as its iteration variable. Sorting by $>_{S}$ is implied by this and condition (4.22) (for elements of different degrees) and the sorting of $S$ by $>_{S}$ in step 5 (for elements of the same degree).

6 . For each $k, S$ is finite, so there are only finitely many steps to take.

Since these properties of the algorithm PHall and its variations are central to this chapter, we introduce a corresponding definition:

Definition 4.3.4 (pseudo-Hall-basis). Let $\mathrm{G}$ be a graded Lie algebra generated by the set $X$. A tuple $(H, B)$ with $H \subset M(X) \subset L(X)$ and $B \subset L(X)$ with properties 1$)$ through 4$)$ of the previous theorem is called a pseudo-Hall-basis of $L(X) / \bar{I}_{l_{\max }}$, or, more colloquially, of $\mathrm{G}$ up to degree $l_{\max }$.

Corollary 4.3.5. Obviously, $\mathrm{PHall}\left(\mathrm{X}, l_{\max }\right)$ is a pseudo-Hall-basis of $\mathrm{G}$ up to degree $l_{\max }$, justifying the algorithm's name.

Remark 4.3.6. The order $>_{S}$ used in step 5 determines the choice of the bases $H_{l}$ of $L_{l}$, and therefore it also determines the elements that will be considered as basis elements in later steps. An obvious possible choice for $>_{S}$ is the Hall order $>_{H}$. An minor advantage of using $>_{S}=>_{H}$ is that elements $\left(h^{\prime}, h^{\prime \prime}\right) \in M(X)$ with $h^{\prime} \leq h^{\prime \prime}$ (that do not need to be considered for $S$ because they are exactly those that fail the test in step 3 of IsHallElement without changing $H)$ can be omitted in the computation of $S$, which means that step 3 of IsHallElement can be omitted as well.

Note that any order $>$ that satisfies $\operatorname{deg}_{\mathrm{H}} m>\operatorname{deg}_{\mathrm{H}} n \Rightarrow m>n$ is a Hall order (this is example 4.2 .83 . In other words, any sorting of $S$ in each step of the algorithm can be produced by 
choosing a corresponding Hall order. But since the Hall order also determines which one of the elements of $M(X)$ whose images in $G$ are linearly dependant due to alternativity and Jacobi identity are suppressed, a user of this algorithm might have reasons to choose $>_{S} \neq>_{H}$ for certain applications.

Remark 4.3.7. Note that $B_{l}$ is not a linear basis of $\bar{I}_{l} \cap \mathrm{G}_{l}$. Such a basis could be found by using a the intersection of $G_{l}$ with a Hall set of $L(X)$ instead of $S$ in the algorithm. But by design of the algorithm PHall, $S$ is linearly independent in $L(X) / \bar{B}_{l-1}$, so it can contain fewer elements.

We can try to minimize the number of elements $\# S$ the following way. Depending on $<_{H}$, different Hall words can be subwords of different numbers of Hall words of a given higher - monomial degree. Therefore, for a given degree $l$, finding a basis of $L(X) / \bar{I}_{l}$ (which is controlled by $<_{S}$ ) consisting of Hall words that are subwords of few longer Hall words means that more relations between the elements of $L(X)$ are already contained in the ideal $\bar{I}_{l}$ generated by $\bar{B}_{l}$. This in turn reduces \#S, and with it size of the output of PHall as well as the computational cost.

Of course, this reduction is not necessarily the most important objective one follows. It is also possible to choose $<_{S}$ and $<_{H}$ in such a way that $H$ and $B$ have other desired properties. An example for different choices of $<_{S}$ will be given below in example 4.4.5.

Remark 4.3.8. $B$ is computed by the algorithm PHall, but it is not used as an input anywhere within it. So if the relations in $G$ are not of concern for our computation, solving the equation in step 8 for coefficients $c_{i j}$ can be omitted. Since this calculation is more computationally expensive than simply checking for linear independence, this can save a considerable amount of time. One example of a computation where only $H$ is important is a check if some finite subset $X \in G$ generates $G$ when a formula for $\operatorname{dim} G_{l}$ is known. If $\operatorname{dim} G_{l}=\# H_{l}$ for all $l=1, \ldots, l_{\max }$, then $X$ generates $G$ up to stratum $l_{\max }$ (more formally, $X$ generates the Lie algebra $L(X) / \bar{I}_{l_{\max }}$, whose linear subspace $\bar{L}_{l_{\max }}$ is isomorphic to $\bar{G}_{l_{\max }}$ as a vector space).

Remark 4.3.9. In practice, a major part of the actual running time of the algorithm is spent on the linear algebra operations in steps 7 and 8 because the dimensions of the vector spaces spanned by $H, B$ can be quite large (an upper limit is given by WITT's formula 4.2.13, which expresses the dimension in the free case). If it is known that no relations can exist between certain elements of $G, S$ can be split into smaller subsets on which the linear operations can, depending on the problem, be significantly faster. In practice, it is useful to compute $B_{l}$ in a separate step after the corresponding $H_{l}$ have been computed because this way, only a single matrix has to be inverted, and all other matrix operations are just multiplications. See appendix A.3.2 for a practical implementation.

Example 4.3.10. If all relations in $\mathrm{G}$ are homogeneous, in step 7 of the algorithm, only linear independence of $S_{i}$ from $H_{l}:=\{h \in H \mid \operatorname{deg} h=l\}$ (instead of $H$ ) has to be checked.

Example 4.3.11 (PHall in free Lie algebras). In the case of a free Lie algebra, the entire set $S$ is linearly independent by theorem 4.2.12, so the check in step 7 can be omitted. The output $H$ is the set of all Hall elements up to degree $l_{\max }$, and $B$ remains empty throughout the entire algorithm. Removing the now reduntant parts of PHall, we obtain Hall, which in this sense is a special case of PHall.

To conclude this section, we give an example of the results of PHall, which can be generalized. 
Example 4.3.12 ( $\mathrm{PHall}$ in $\mathfrak{s l}_{2}$ ). Consider the Lie algebra $\mathfrak{s l}_{2}$ with Chevalley generators $x, y, h$. Let $>_{H}$ and $>_{S}$ be DegLex and $l_{\max } \geq 2$. In step $l=2$, the algorithm produces $S=([y, x],[h, x])[h, y])$, which are all linearly dependent of $x, y, h$, to be more precise

$$
B_{2}=([y, x]-h,[h, x]-2 x,[h, y]+2 y) .
$$

Further, $H_{l}=()$ for $l>1$ and $B_{l}=()$ for $l>2$, so $H=(x, y, h)$ and $B=B_{2}$ for the following general reason:

Remark 4.3.13 (PHall in finite dimensional Lie algebras). For any finite-dimensional Lie algebra $G$, we can always find a $l_{\max , 0}$ large enough such that in the algorithm PHall, the set $\bar{H}_{l_{\max , 0}}$ spans all of $\mathrm{G}$ (otherwise we would have infinitely many elements of $H$ which are linearly independent). This implies $H_{l}=\{\}$ for $l>l_{\max , 0}$. Note that since elements of $S$ are Lie brackets of elements of $H$, the list $S$ remains empty for any $l>2 l_{\max , 0}$, so we also have $B_{l}=\{\}$ for $l>2 l_{\max , 0}$.

This also means that in the case of a finite-dimensional Lie algebra of known dimension, we can replace the exit condition of the loop over $l$ of the algorithm PHall by a check if $\# H=\operatorname{dim} G$, noting down $l_{\max , 0}:=l$, and continuing until $l=\min \left(l_{\max }, 2 l_{\max , 0}\right)$. An advantage of this is that the computer will never go through more iterations of the loop in PHall than necessary, at the cost of introducing another slightly altered variant of the Hall algorithm.

Remark 4.3.14 (Hall sets in HAPPLE's work on the Poisson algebra of invariant charges). The idea of using Hall sets this way to find the relations in a non-free lie algebra suggests itself. Hall sets have even been used in the narrow context of the Poisson algebra $\mathfrak{h}$ of invariants of the Nambu-Goto string (cf. 3.2.2). A major part of HAPPLE's doctoral dissertation about this Poisson algebra [Hap93] consisted in using a method that contained a similar approach to find all the relations in the Poisson algebra of invariant charges for $d=3$ up to $l=5$.

Following conjecture 3.2 .8 that $\mathfrak{h}_{m}=\mathfrak{a} \ltimes \mathfrak{U}$, HAPPLE uses as his generators a set of generators of the Poisson subalgebra $\mathfrak{U}$ (given in detail in remark 3.2.10, namely the element $Q$, which is a basis of the one-dimensional stratum $\mathfrak{h}_{m 0}$, and the basis elements $L_{i}, i=-2,-1,0,1,2$ of $\left.\mathfrak{h}_{m 1}\right)$ and the exceptional element $L_{(3)} \in \mathfrak{h}_{3}$, which is the only exceptional element that has relations of degree $\leq 5$.

Due to the Leibniz identity, all elements of a Poisson algebra can be written as sums of products of Poisson brackets of the Poisson algebra's generators.

Similarly to the algorithms in this section, HAPPLE's general idea is to use the fact that the Hall words of these generators are a basis of all their multiple Poisson brackets (this is theorem 4.2.12). One can then calculate all the different products of these Hall words; due to commutativity of the product, one can write them down in a standard order (HAPple uses DegLex) and thus obtain a set spanning $\mathfrak{h}$.

Again similar to all the different versions of the Hall algorithm given in this thesis, one can now choose a set of linearly independent products of Hall words and express all other products of Hall words as linear combinations (HAPple uses a modification of the Jordan algorithm [Hap93, p. 160f]). The relations that are not contained in the ideal generated by the relations of lower strata are called the "new relations".

In comparison, HAPPLE's problem of finding all relations of generators in the Poisson algebra is more complicated than the problem considered in this thesis of finding the relations of generators of a Lie algebra (i.e. there is no product beyond the Lie bracket), which is why 
the Hall basis is not given a particularly prominent role in HAPPLE's thesis. In particular, not much consideration was given to the role the Hall order plays in that thesis, or which ones of the linearly dependent elements of the Hall basis to choose for the continuing computations (which is treated herein by the introduction of the sorting order $<_{s}$ ).

In addition, the special case of the Poisson algebra of invariants for $d=3$ considered by HAPPLE allows for some significant simplifications which only have an analogue for the Pohlmeyer-Rehren Lie algebra for $d=2$ that was not considered here:

- By construction, all multiple Lie brackets of the generators used by HAPPLE are eigenvectors of adjunction of $Q$ (the magnetic quantum number being the corresponding eigenvalue), so a Hall word of the form $[Q, H]$ where $H$ is another Hall word of the generators is linearly dependent of $H$. HAPPLE presupposes these relations and does not use Hall words containing $Q$ other than $Q$ itself (as a Hall word of length 1 ).

Happle can also consider exceptional elements separately (cf. [Hap93, p. 66]). With these exceptions, all generators are of degree $l=1$. In other words, their degree is equal to their monomial degree, and Happle does not have a reason to consider any other cases, as it is done in sections 4.4 and 4.5 .

- Analogous to $\mathfrak{g}$, the Poisson algebra of invariants $\mathfrak{h}_{m}$, taken as a Lie algebra, is graded and can be decomposed into multiplets, which are irreducible modules of the stratum $\mathfrak{h}_{m 0}$, which in turn is isomorphic to $\mathfrak{s} \mathfrak{d}(d-1, \mathbb{C})$ (note the difference to $\mathfrak{g}_{0}$, which is isomorphic to $\mathfrak{s} \mathfrak{v}(d, \mathbb{C})$ ).

However, another consequence of HAPPLE's precondition of $d=3$ is that $\mathfrak{h}_{0}$ is onedimensional, and so are all its irreducible modules (there are no ladder operators). So no interesting multiplet structure exists for $d=3$, and HAPpLE has no reason to give special consideration to generators of degree 0 like the ladder operators, as it is done in 4.5 .

Furthermore, there is no clear concisive account known to the author of this thesis of concrete algorithms in the general context of ideals in Lie algebras, which was a further motivation for writing this chapter.

Remark 4.3.15 (self-reducedness and Gröbner bases). By theorem 4.3.3, the algorithm PHall finds self-reduced sets $\bar{B}_{k}$ generating the ideals $\bar{I}_{k}$.

In the general situation of a subset $\mathcal{G} \in L(X)$ such that $\mathcal{G}$ generates an ideal $I$, we call $\mathcal{G}$ a Gröbner basis of $I$ if for all $f \in I$, there is a $g \in \mathcal{G}$ such that $\operatorname{LM}(g)$ is a factor of $\operatorname{LM}(f)]^{8}$ Gröbner bases are an important tool in the field of computational algebra. For instance, they allow to efficiently compute if two elements $f, g \in L(X)$ are identical modulo to $I$ (this is sometimes called the word problem).

A theorem due to SHIRshov (see [dG00, 7.10.2]) leads to an explicit criterion ([dG00, 7.10.4]) for the conditions under which a self-reduced set generating an ideal is a Gröbner basis. However, in the case of infinite-dimensional Lie algebras such as the PohlmeyerRehren Lie algebra that this thesis is focused on, this criterion cannot be checked by explicit computations (that could be done by computer algebra) in finite time.

\footnotetext{
${ }^{8}$ Definition taken from [dG00, 7.10], also see [Ufn98] for a discussion of Gröbner bases in noncommutative algebras.
} 
De GraAf also gives an algorithm [dG00, GröbnerBasis, p. 297] that finds a Gröbner basis of an ideal generated by a finite set.

Finally, in the case of the Pohlmeyer-Rehren Lie algebra, even if a generating set $\bar{H}_{l}$ of one of the ideals $\bar{I}_{l}$ could be shown to be a Gröbner basis, $\bar{H}_{l}$ would not be a generating set of $I_{l+1}$, much less of $\bar{I}_{l}$, because a comparison of dimensions using theorem 1.3.6 shows that for each $l$, relations contained in $I_{l+1} \subset \bar{I}_{l+1}$ but not contained in $\bar{I}_{l}$ must exist. Therefore, this avenue of research has not been pursued further.

Before we apply the algorithm to the motivating Pohlmeyer-Rehren Lie algebra, we can consider a much simpler infinite-dimensional Lie algebra, the Witt algebra, to provide some intuitions.

Definition 4.3.16 (Witt algebra). The linear span of the elements $L_{n}, n \in \mathbb{Z}$ over $\mathbb{C}$ with the bracket

$$
\left[L_{m}, L_{n}\right]:=(m-n) L_{m+n}
$$

is a Lie algebra, called the Witt algebra $\mathfrak{w}$. It is obviously $\mathbb{Z}$-graded (by $n$ ), and its strata are one-dimensional;

$$
\mathfrak{w}_{n}=\mathbb{C} \cdot L_{n} .
$$

We can also consider subagebras of the Witt algebra that are generated by a finite set of elements. For instance, one can easily show by induction over $n$ (iteratively adjoining further copies of $L_{1}$ to $L_{2}$ ) that the elements $L_{1}$ and $L_{2}$ generate the subalgebra of elements of positive degree.

Example 4.3.17. We compute the first few steps of PHall for the subalgebra of $\mathfrak{w}$ generated by $L_{1}$ and $L_{2}$, using $<_{H}=<_{S}=$ DegLex with $L_{1}<L_{2}$ (cf. example 4.2.14).

\begin{tabular}{|cc|c|cc|}
\hline$k$ & $i$ & $s_{i} \in S$ (Hall element) & $s_{i}$ (standard basis) & lin. dep. of $H$ \\
\hline 2 & 1 & {$\left[L_{1}, L_{2}\right]$} & $-L_{3}$ & no \\
\hline 3 & 1 & {$\left[L_{1},\left[L_{1}, L_{2}\right]\right]$} & $2 L_{4}$ & no \\
3 & 2 & {$\left[L_{2},\left[L_{1}, L_{2}\right]\right]$} & $L_{5}$ & no \\
\hline 4 & 1 & {$\left[L_{1},\left[L_{1},\left[L_{1}, L_{2}\right]\right]\right]$} & $-6 L_{5}$ & yes \\
4 & 2 & {$\left[L_{2},\left[L_{1},\left[L_{1}, L_{2}\right]\right]\right]$} & $-4 L_{6}$ & no \\
4 & 3 & {$\left[L_{2},\left[L_{2},\left[L_{1}, L_{2}\right]\right]\right]$} & $-3 L_{7}$ & no \\
\hline 5 & 1 & {$\left[L_{2},\left[L_{1},\left[L_{1},\left[L_{1}, L_{2}\right]\right]\right]\right]$} & $24 L_{6}$ & yes \\
5 & 2 & {$\left[L_{2},\left[L_{2},\left[L_{1},\left[L_{1}, L_{2}\right]\right]\right]\right]$} & $16 L_{8}$ & no \\
5 & 3 & {$\left[L_{2},\left[L_{2},\left[L_{2},\left[L_{1}, L_{2}\right]\right]\right]\right]$} & $15 L_{9}$ & no \\
5 & 4 & {$\left[\left[L_{1}, L_{2}\right],\left[L_{1},\left[L_{1}, L_{2}\right]\right]\right]$} & $2 L_{7}$ & yes \\
5 & 5 & {$\left[\left[L_{1}, L_{2}\right],\left[L_{2},\left[L_{1}, L_{2}\right]\right]\right]$} & $2 L_{8}$ & yes \\
\hline
\end{tabular}

Notice that in step $k=4, i=1$, we obtain the first relation, $\left[L_{1},\left[L_{1},\left[L_{1}, L_{2}\right]\right]\right]+6\left[L_{2},\left[L_{1}, L_{2}\right]\right]=0$. A consequence of this is that the left summand is not an element of $S$. Since the right summand has lower degree than the left one, this does not depend on $<_{s}$. A further consequence of this is that the word $\left[L_{1},\left[L_{1},\left[L_{1},\left[L_{1}, L_{2}\right]\right]\right]\right]$ (while being a Hall word) is not an element of $S$. Also notice that for $k=5$, not only the order of the list $S$ but also its contents depends on $<_{S}$; if we had used another order $<_{S}$ such that

$$
\left[L_{2},\left[L_{2},\left[L_{1},\left[L_{1}, L_{2}\right]\right]\right]\right]>_{S}\left[\left[L_{1}, L_{2}\right],\left[L_{2},\left[L_{1}, L_{2}\right]\right]\right],
$$

then the element on the right hand side would have been included in $S$ instead of the one on the left hand side. 


\subsubsection{Application to the Pohlmeyer-Rehren Lie algebra}

Using the Mathematica module PHall given in appendix A.3.1, and setting

$$
<_{H}=<_{S}=\text { DegLex },
$$

the following results can be obtained.

For $d=3$ :

- Using as $X$ the basis (of dimension 8) of $\mathfrak{g}_{1}$ uniquely defined up to scalars by weight and multiplet (cf. remark 2.3.17 for the concrete values used), the lists $H_{l}$ and $B_{l}$ show the following growth up to the limit of computation within a few hours 9 .

\begin{tabular}{|l|rrrrr|}
\hline$l$ & 2 & 3 & 4 & 5 & 6 \\
\hline$\# H_{l}$ & 18 & 48 & 116 & 312 & 810 \\
$\# B_{l}$ & 10 & 66 & 216 & 571 & 1910 \\
$\operatorname{dim}_{l}$ & 18 & 48 & 116 & 312 & 810 \\
$t_{\text {comp }}[s]$ & 0.032 & 0.99 & 21.0 & 547 & $21.7 \mathrm{k}$ \\
NumLyndon $(l, 8)$ & 28 & 168 & 1008 & 6552 & 43596 \\
\hline
\end{tabular}

For reference $\operatorname{dim} \mathfrak{g}_{l}$, calculated by using theorem 1.3.6, as well as $t_{\text {comp }}$, the time to compute the given iteration for $l$ and NumLyndon $(l, 8)$, the number of elements of a Hall basis of degree $l$, are included.

- By comparing the lines giving $\# H_{l}$ and $\operatorname{dim} \mathfrak{g}_{l}$, we conclude that $\mathfrak{g}_{1}$ generates $\mathfrak{g}_{2}, \ldots, \mathfrak{g}_{6}$, consistent with conjecture 4.1.2.

- The sets $X \backslash\{v\}$ with $v \in\left\{{ }^{2} v^{2},{ }^{2} v^{1},{ }^{2} v^{-1},{ }^{2} v^{-1}\right\}$ do not generate $g_{2}$, so a forteriori they do not generate $\mathrm{g}$.

- All other subsets of $X$ containing seven elements generate $\mathfrak{g}_{2}$.

For $d=4$,

- Using as $X$ the basis (of dimension 20) of $\mathfrak{g}_{1}$ uniquely defined up to scalars by weight and multiplet (cf. remark2.3.17 for concrete values), the algorithm performs as follows:

\begin{tabular}{|l|rrrr|}
\hline$l$ & 2 & 3 & 4 & 5 \\
\hline$\# H_{l}$ & 60 & 204 & 670 & 2340 \\
$\# B_{l}$ & 130 & 757 & 2965 & 10014 \\
$\operatorname{dim} \mathfrak{g}_{l}$ & 60 & 204 & 670 & 2340 \\
$t_{\text {comp }}[s]$ & 0.84 & 42.6 & $18.1 \mathrm{k}$ & $124 \mathrm{k}$ \\
NumLyndon $(l, 20)$ & 190 & 2660 & 39900 & 639996 \\
\hline
\end{tabular}

- Again, the results are consistent with conjecture 4.1.2.

- Any subset of $X$ containing at least 18 elements generates $\mathfrak{g}_{2}$, but some subsets of $X$ containing 17 elements don't.

\footnotetext{
${ }^{9}$ All runtime data taken on the same Intel Core i7-3770K (4 CPU cores with hyperthreading at $3.5 \mathrm{GHz}$ ) with $32 \mathrm{GiB}$ of RAM running Linux.
} 
These findings illustrate why self-reducedness of $B$ is an important property of the algorithm: if we had begun by computing a Hall basis of $L(X)$ and then found the relations between them, NumLyndon $(l, \# X)$ elements would have had to be considered for extension to a basis. In the case of $d=4, l=5$, this would have entailed more than 50 times as many calculations as were needed using the algorithm PHall.

A similar consideration also indicates that we might be able to test the conjectures at the beginning of this chapter for larger $l$ if we use a smaller generating set than a basis of $\mathfrak{g}_{1}$. An obvious idea is to only use the highest (or lowest) weight vectors in $\mathfrak{g}_{1}$ and enough elements of $\mathfrak{g}_{0}$ so that the entirety of $\mathfrak{g}_{1}$ is generated. The rest of the chapter will deal with modifications to the Philip Hall algorithm that are tailored to this objective.

\subsection{Generation in order of an external, positive gradation}

So far our algorithm has generated the elements of a pseudo-Hall-basis of a Lie algebra $G$ inductively with a loop using the monomial degree as its counter. This is straightforward and convenient in some applications, but impractical in others:

- For instance, G might already be equipped with a different gradation, and we would want our output to be exactly all elements of the Pseudo-Hall-basis up to a given degree, sorted by degree (regarding that gradation).

- We might want to drop the restiction that $\# X$, the number of generators, is finite. But then, the number of brackets that have to be considered in the very first step (i.e. $l=2$ ) is infinite and the algorithm will never terminate. A strategy we could pursue here would be to equip $X$ with a gradation such that $X$ is degreewise finite, so that there are only finitely many generators of each degree that have to be considered within each iteration of the loop corresponding to a degree.

Taking this into account, for this section let G be Lie algebra that is graded with a $\mathbb{N}_{+}-$ $\operatorname{gradation}_{\mathrm{deg}}$ and generated by a degreewise finite set $X$. We will call $\mathrm{deg}_{\mathrm{e}}$ external gradation in the sequel to distinguish it from the monomial degree $\mathrm{deg}_{\mathrm{H}}$. As before, let $<_{S}$ be a total order on $M(X)$ and $<_{H}$ be a Hall order on $M(X)$.

Note that this implies that that $G$ is degreewise finite dimensional, i.e. $\operatorname{dim} G_{l}<\infty$ for all $l \in \mathbb{N}_{+}$, because by theorem 4.2.4. $G$ is a quotient algebra of the free Lie algebra $L(X)$, which has to be degreewise finite dimensional with respect to deg $_{\mathrm{e}}$.

To modify the algorithm accordingly, two aspects have to be taken into consideration.

Remark 4.4.1. Since elements of $X$ can have any degree now, instead of being considered only once during the initialization (in step 1 of PHall), they need to be included in $S$ in every step of the iteration over $l$ (which is done in step 4).

Remark 4.4.2. The requirement that $\operatorname{deg}_{\mathrm{e}}$ is strictly positive implies

$$
\operatorname{deg}_{\mathrm{e}}(m, n)=\operatorname{deg}_{\mathrm{e}}(m)+\operatorname{deg}_{\mathrm{e}}(n)>\max \left(\operatorname{deg}_{\mathrm{e}} m+\operatorname{deg}_{\mathrm{e}} n\right) .
$$

This implies that in step $l$, the arguments of the Lie brackets considered for $S$ are of strictly lower degree than $l$, and, as can be shown by induction over $l$, are elements of $\bar{H}_{l-1}$. If this condition is dropped, further modifications to the algorithm will have to be made as lined out in the next section. 
Considering this, we obtain the following modified algorithm. The input are the set $X$ of generators of $\mathrm{G}$ and a maximal (external) degree $l_{\max }$ to compute up to. The output are ordered tuples $H$ and $B$.

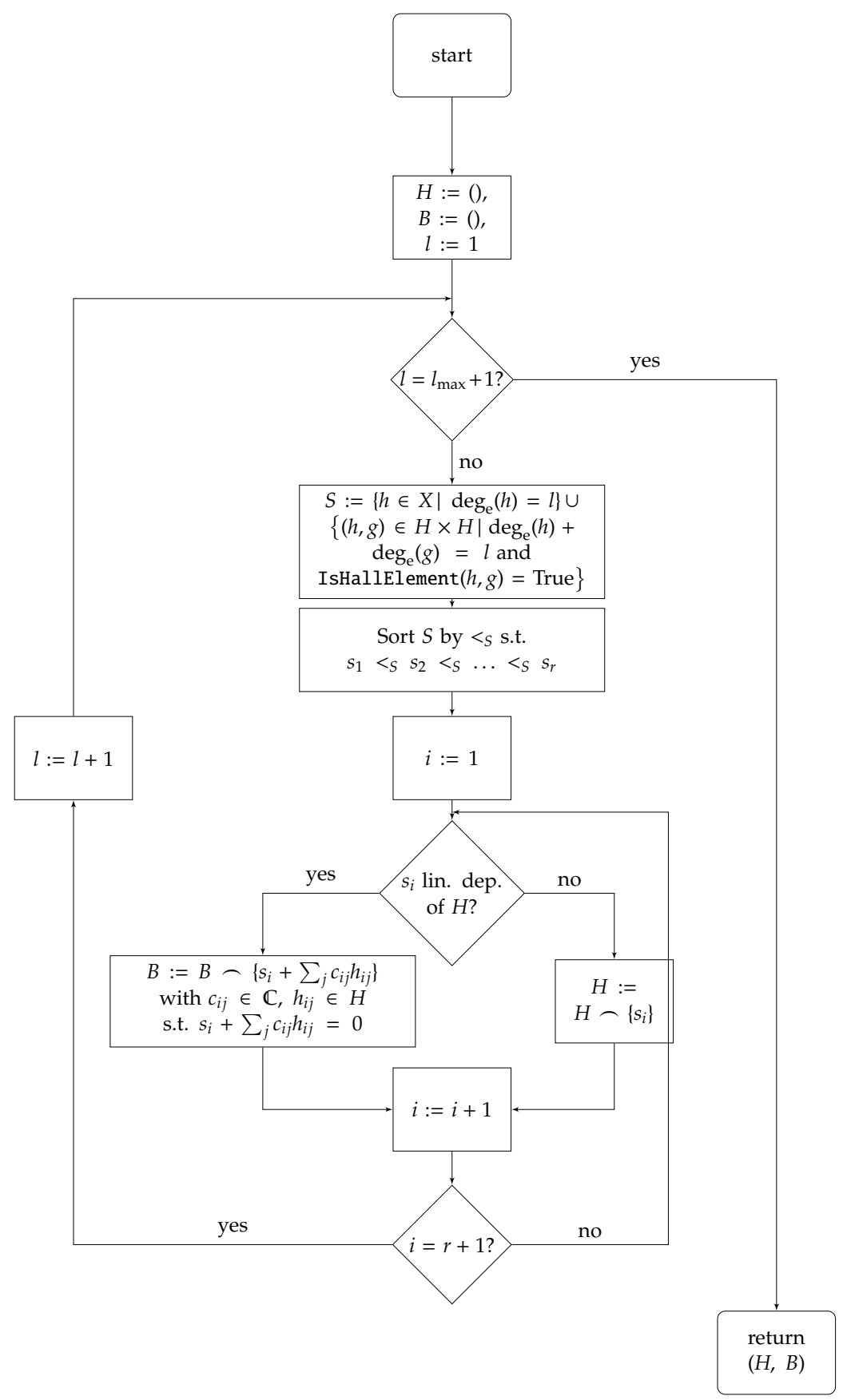

Figure 4.4: Flow chart of algorithm PHallPosExtGrad 


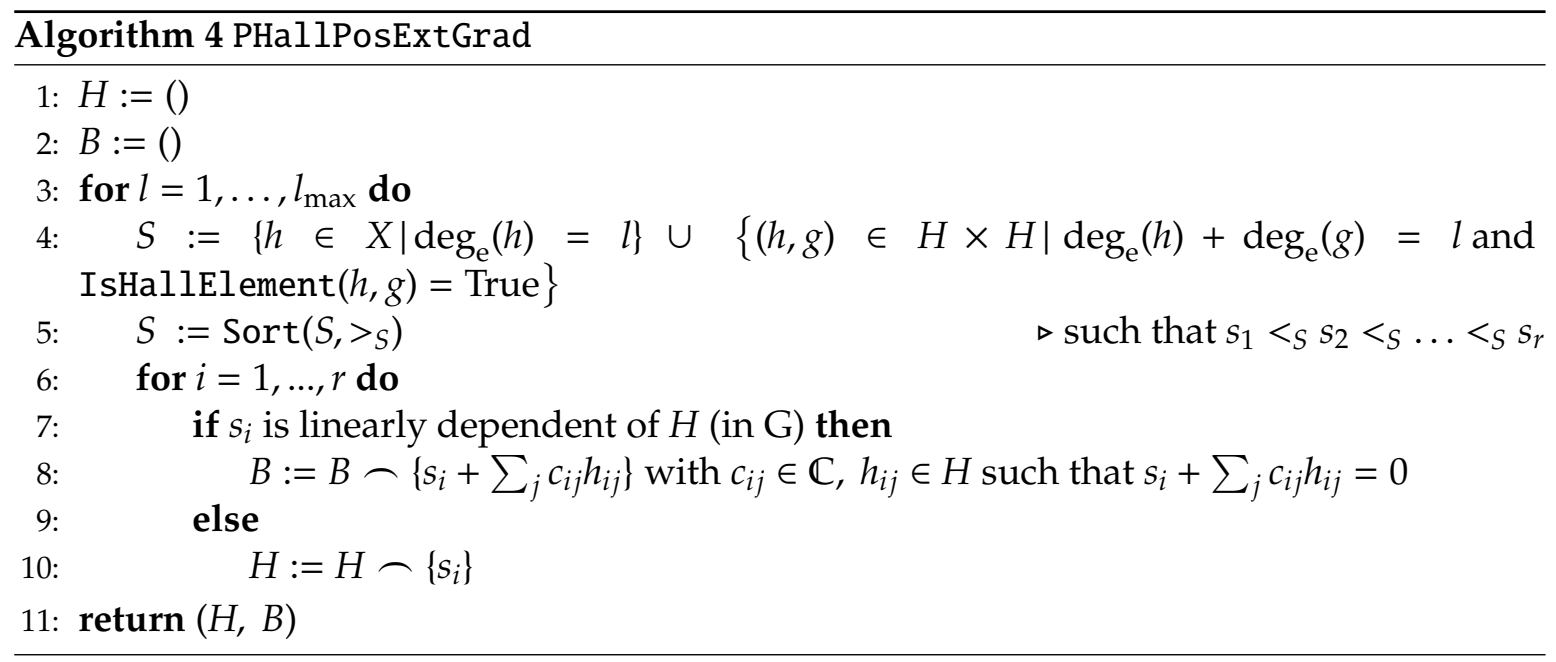

Remark 4.4.3. Theorem 4.3.3 and its proof still hold (with deg $=\mathrm{deg}_{\mathrm{e}}$ ), so $(H, B)=$ PHallPosExtGrad $\left(X, l_{\max }\right)$ is a pseudo-Hall-basis of $G$ up to external degree $l_{\max }$.

Remark 4.4.4. If the external degree used is the monomial degree, then $\mathrm{G}_{0}=\{\}$, and the algorithm coincides with the algorithm PHall for $l>1$.

Example 4.4.5. We compute the first few steps of PHallPosExtGrad for the $\left(\mathbb{N}_{+}\right.$-graded) subalgebra of the Witt algebra $\mathfrak{w}$ (def. 4.3.16) that is generated by $L_{1}$ and $L_{2}$, using $<_{H}=<_{S}$ DegLex with $L_{1}<L_{2}$ (cf. example 4.2.14 and 4.3.17).

\begin{tabular}{|cc|c|cc|}
\hline$l$ & $i$ & $s_{i}$ (Hall element) & $s_{i}$ (standard basis) & lin. dep. of $H$ \\
\hline 1 & 1 & $L_{1}$ & $L_{1}$ & no \\
\hline 2 & 1 & $L_{2}$ & $L_{2}$ & no \\
\hline 3 & 1 & {$\left[L_{1}, L_{2}\right]$} & $-L_{3}$ & no \\
\hline 4 & 1 & {$\left[L_{1},\left[L_{1}, L_{2}\right]\right]$} & $2 L_{4}$ & no \\
\hline 5 & 1 & {$\left[L_{2},\left[L_{1}, L_{2}\right]\right]$} & $L_{5}$ & no \\
5 & 2 & {$\left[L_{1},\left[L_{1},\left[L_{1}, L_{2}\right]\right]\right]$} & $-6 L_{5}$ & yes \\
\hline 6 & 1 & {$\left[L_{2},\left[L_{1},\left[L_{1}, L_{2}\right]\right]\right]$} & $-4 L_{6}$ & no \\
\hline 7 & 1 & {$\left[L_{2},\left[L_{2},\left[L_{1}, L_{2}\right]\right]\right]$} & $-3 L_{7}$ & no \\
7 & 2 & {$\left[\left[L_{1}, L_{2}\right],\left[L_{1},\left[L_{1}, L_{2}\right]\right]\right]$} & $2 L_{7}$ & yes \\
\hline
\end{tabular}

For contrast, if we use another sorting order, $<_{S}=$ Lex, we find

\begin{tabular}{|cc|c|cc|}
\hline$l$ & $i$ & $s_{i}$ (Hall element) & $s_{i}$ (standard basis) & lin. dep. of $H$ \\
\hline 1 & 1 & $L_{1}$ & $L_{1}$ & no \\
\hline 2 & 1 & $L_{2}$ & $L_{2}$ & no \\
\hline 3 & 1 & {$\left[L_{1}, L_{2}\right]$} & $-L_{3}$ & no \\
\hline 4 & 1 & {$\left[L_{1},\left[L_{1}, L_{2}\right]\right]$} & $2 L_{4}$ & no \\
\hline 5 & 1 & {$\left[L_{1},\left[L_{1},\left[L_{1}, L_{2}\right]\right]\right]$} & $-6 L_{5}$ & no \\
5 & 2 & {$\left[L_{2},\left[L_{1}, L_{2}\right]\right]$} & $L_{5}$ & yes \\
\hline 6 & 1 & {$\left[L_{1},\left[L_{1},\left[L_{1},\left[L_{1}, L_{2}\right]\right]\right]\right]$} & $24 L_{6}$ & no \\
6 & 2 & {$\left[L_{2},\left[L_{1},\left[L_{1}, L_{2}\right]\right]\right]$} & $-4 L_{6}$ & yes \\
\hline 7 & 1 & {$\left[L_{1},\left[L_{1},\left[L_{1},\left[L_{1},\left[L_{1}, L_{2}\right]\right]\right]\right]\right]$} & $-120 L_{7}$ & no \\
7 & 2 & {$\left[\left[L_{1}, L_{2}\right],\left[L_{1},\left[L_{1}, L_{2}\right]\right]\right]$} & $2 L_{7}$ & yes \\
7 & 3 & {$\left[L_{2},\left[L_{1},\left[L_{1},\left[L_{1}, L_{2}\right]\right]\right]\right]$} & $18 L_{7}$ & yes \\
\hline
\end{tabular}


Comparing the two results, it is apparent that not only the output depends on $<_{S}$, but also the number of computations required (cf. remark 4.3.7).

Remark 4.4.6. If $\mathrm{G}$ is a degreewise finite dimensional graded Lie algebra, any basis $b_{\mathrm{G}}$ of $\mathrm{G}$ satisfies the requirements for $X$ in this chapter. In conjunction with a slight modification of the algorithm given above, this can be used the following way (that emphasizes the practical importance of the choice of $<_{S}$ ) to iteratively expand a tuple $Y$ of elements of $G$ so that it generates $\overline{\mathrm{G}}_{l}$ with increasing $l$ :

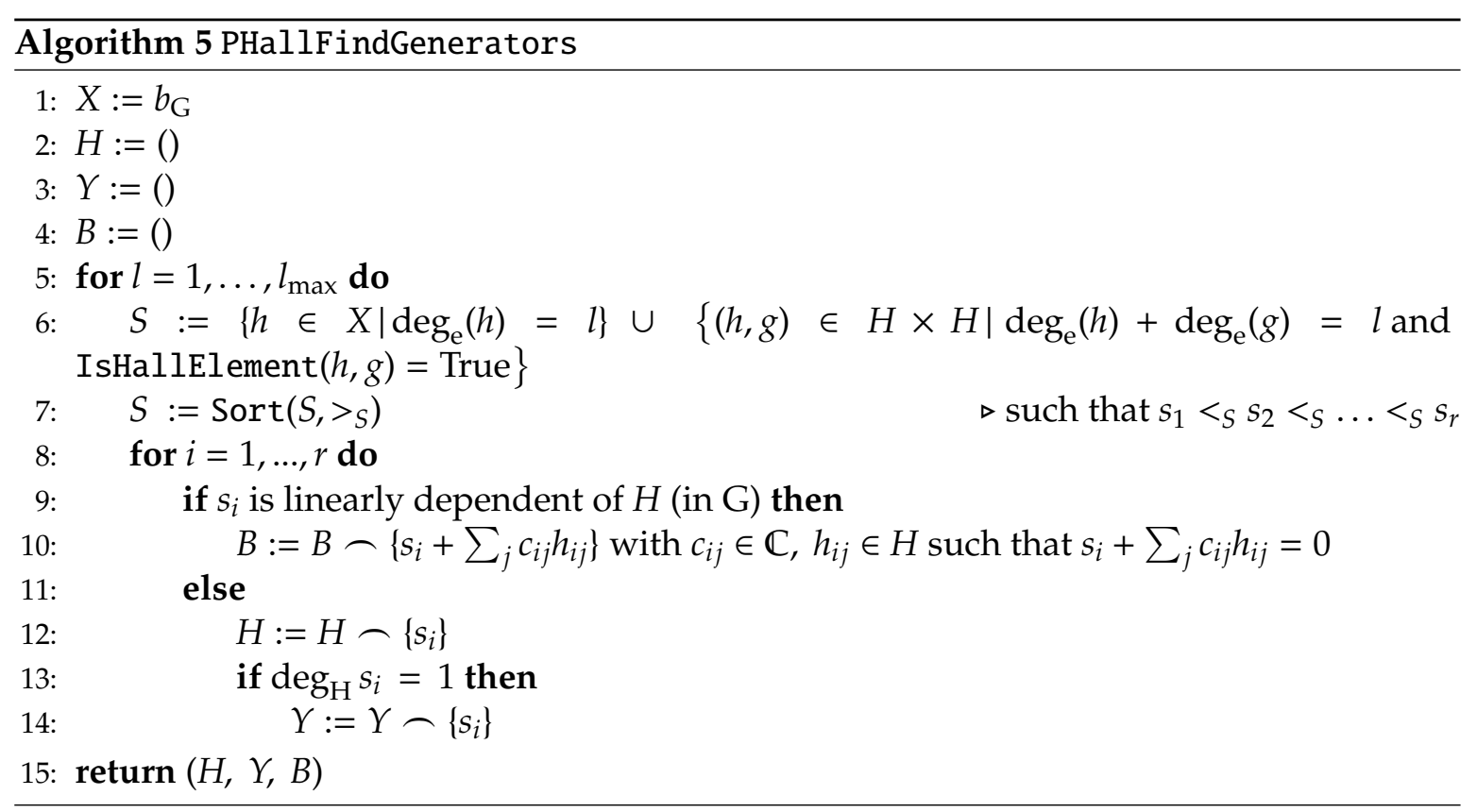

By theorem 4.3.3, if we run the algorithm, we obtain a basis $H$ of $\bar{L}_{l_{\max }}$ (the fact that $b_{\mathrm{G}}$ may be infinite is not a problem because the algorithm only ever considers one stratum at a time which is finite dimensional). Because every element of $H$ that has monomial degree 1 is added to $Y$, the tuple $Y$ generates $H$, and with it $\bar{L}_{l_{\max }}$.

Now $H$ depends on the sorting order $<_{S}$ used in the algorithm. If $<_{S}$ satisfies $g<_{S} h$ for all $g, h \in \mathrm{G}$ with $\operatorname{deg}_{\mathrm{H}} g<\operatorname{deg}_{\mathrm{H}} h$, then $Y$ will be kept minimal in the sense that elements of $b_{\mathrm{G}}$ will only be added to $Y$ if they are not in the subalgebra generated by the previous elements of $Y$.

If, on the contrary, we choose a sorting order $<_{S}$ that reverses the inequalities given above, $H$ and $Y$ will reproduce $b_{\mathrm{G}}$ and $B$ will be a list of the structure constants of $\mathrm{G}$ (both up to degree $l_{\max }$ ).

\subsubsection{Application: Observations about the multiplet ${ }^{1} \mathfrak{g}_{1}$}

In section 2.4, we had seen that the multiplet ${ }^{1} \mathfrak{g}_{1}$ might be of relevance to the structure of $\mathfrak{g}$ for $d=3$. Therefore, giving a little attention to the subalgebra generated by ${ }^{1} \mathfrak{g}_{1}$ is warranted. Call this subalgebra $F$. When we use the algorithm PHallPosExtGrad to explore the structure of $F$, we find that for each $l$ in $\{2, \ldots, 8\}$ - this is the limit of computation on the machine used $-H_{l}$ consists of one element of magnetic quantum numbers $0, \pm 1$ each, and because of lemma 2.4.1. they must form a multiplet of spin 1 . This can be generalized to a conjecture: 
Conjecture 4.4.7. For all $l \in \mathbb{N}$,

$$
F \cap \mathfrak{g}_{l} \cong{ }^{1} V
$$

We also find that $B_{l}$ consists of monomials in the elements of ${ }^{1} \mathfrak{g}_{1}$ of a particular form, as opposed to linear combinations of them, which is the general case. We can generalize this to the " $\subset$ " part of another conjecture, using the notion of the centralizer $Z(S)$ of a subset $S$ of a Lie algebra $G$, defined as

$$
Z(S):=\{g \in G \mid[g, s]=0 \text { for all } s \in S\} .
$$

\section{Conjecture 4.4.8.}

$$
\begin{aligned}
Z\left({ }^{1} v^{1}\right) \cap \mathfrak{g}_{l} & =\left\{c \cdot \operatorname{ad}_{1}^{l-1}{ }_{v^{0}} 1 v^{1} \mid c \in \mathbb{C}\right\}, \\
Z\left({ }^{1} v^{0}\right) \cap \mathfrak{g}_{l} & \left.=\left\{c \cdot \operatorname{ad}_{1}^{l-2}{ }_{v^{0}}^{1} v^{-1},{ }^{1} v^{1}\right] \mid c \in \mathbb{C}\right\}, \\
Z\left({ }^{1} v^{-1}\right) \cap \mathfrak{g}_{l} & =\left\{c \cdot \operatorname{ad}_{v_{v^{0}}^{l}}^{l-1} v_{v^{-1}} \mid c \in \mathbb{C}\right\} .
\end{aligned}
$$

These equalities have been verified using the Mathematica program CenterTest (cf. B.6.8) up to $1=6$, the limit of computation.

Remark 4.4.9. While for all 3-dimensional $\mathfrak{s l}_{2}$-modules ${ }^{1} V$, we have $\left[{ }^{1} V,{ }^{1} V\right] \cong \Phi^{1} V$ with the epimophism $\Phi$ as in lemma 2.4.1, the analogue to conjecture 4.4.7 is not necessarily true for every 3-dimensional $\mathfrak{s l}_{2}$-module, not even for every such $\mathfrak{s l}_{2}$-module that occurs in the weight space decomposition of $\mathfrak{g}$. Take for instance the multiplet $W \cong{ }^{1} V$ occurring in $\left[{ }^{2} \mathfrak{g}_{1},{ }^{1} \mathfrak{g}_{1}\right] \cong{ }^{3} V \oplus^{2} V \oplus{ }^{1} V$. A basis of $W$ is given by the elements

$$
\begin{aligned}
& -7 e(000+)+e(0-++)+4 e(0+-+)+9 e(0++-), \\
& 8 e(00-+)+2 e(00+-)+5 e(0-0+)+7 e(--++), \\
& -7 e(000-)+9 e(0--+)+4 e(0-+-)+e(0+--) .
\end{aligned}
$$

Using PHallPosExtGrad, we can calculate that

$$
\operatorname{dim}[[W, W], W]=8 \neq 3=\operatorname{dim}^{1} V .
$$

Remark 4.4.10. If conjecture 4.4.7 is true, then the " $\supset$ " part of the equations (4.28) and (4.30) can be proven for arbitrary $l$ :

$$
\begin{gathered}
Z\left({ }^{1} v^{1}\right) \cap g_{l} \supset\left\{c \cdot \operatorname{ad}_{1}^{l-1} 1_{v^{0}} v^{1} \mid c \in \mathbb{C}\right\}, \\
Z\left({ }^{1} v^{-1}\right) \cap g_{l} \supset\left\{c \cdot \operatorname{ad}_{1 v^{0}}^{l-1} 1_{v^{-1}} \mid c \in \mathbb{C}\right\} .
\end{gathered}
$$

Proof. - The fact that the right hand sides are subsets of $\mathfrak{g}_{l}$ follows from the fact that the right hand sides are multiple brackets of $l$ elements of $\mathfrak{g}_{1}$.

- Both $v:={ }^{1} v^{1}$ and $w:=\operatorname{ad}_{1}^{l-1} 1_{v^{0}} v^{1}$ have magnetic quantum number 1 , so $[v, w] \in \mathfrak{g}_{l}^{2}$. Since conjecture 4.4.7 is supposed to be true, $[v, w]$ is an element in a multiplet of spin 1 . The only way to satisfy these conditions is $[v, w]=0$, which proves 4.31 .

- Analogous for equation (4.32). 


\subsection{Admitting generators of degree zero}

Until now, we have considered Lie algebras with a stricly positive gradation, which had the benefit that the computations that had to be performed for a given stratum $G_{l}$ only depended on results of lower strata. But if we allow elements $g_{i} \in \mathrm{G}_{0}$ then if $h \in \mathrm{G}_{l}$, also $\operatorname{ad}_{g_{1}} h \in \mathrm{G}_{l}$, $\operatorname{ad}_{g_{2}}$ ad $_{g_{1}} h \in \mathrm{G}_{l}$ and so on, so running through a loop with the external degree $l$ as the counter variable only once for each $l$, like the algorithm PHallPosExtGrad does, will fail to produce all these elements. This section will give two strategies to take these elements into account.

In this section, let $\mathrm{G}$ be a Lie algebra with a $\mathbb{N}_{0}$-gradation $\operatorname{deg}_{\mathrm{e}}$ that is degreewise finitedimensional, i.e. $\operatorname{dim} G_{l}<\infty$ for all $l \in \mathbb{N}_{0}$.

\subsubsection{Iterative extension to a basis}

The first strategy consists in iterating the following way: if we find new linearly independent elements of $G$, we need to consider all those elements that can be obtained by adjoining elements of $G_{0}$ to them for our extension to a basis in another step. We repeat this process until no new linearly independent elements are found.

The following algorithm implements this idea, using the list $\tilde{H}$ of newly found basis vectors of $\mathrm{G}$ :

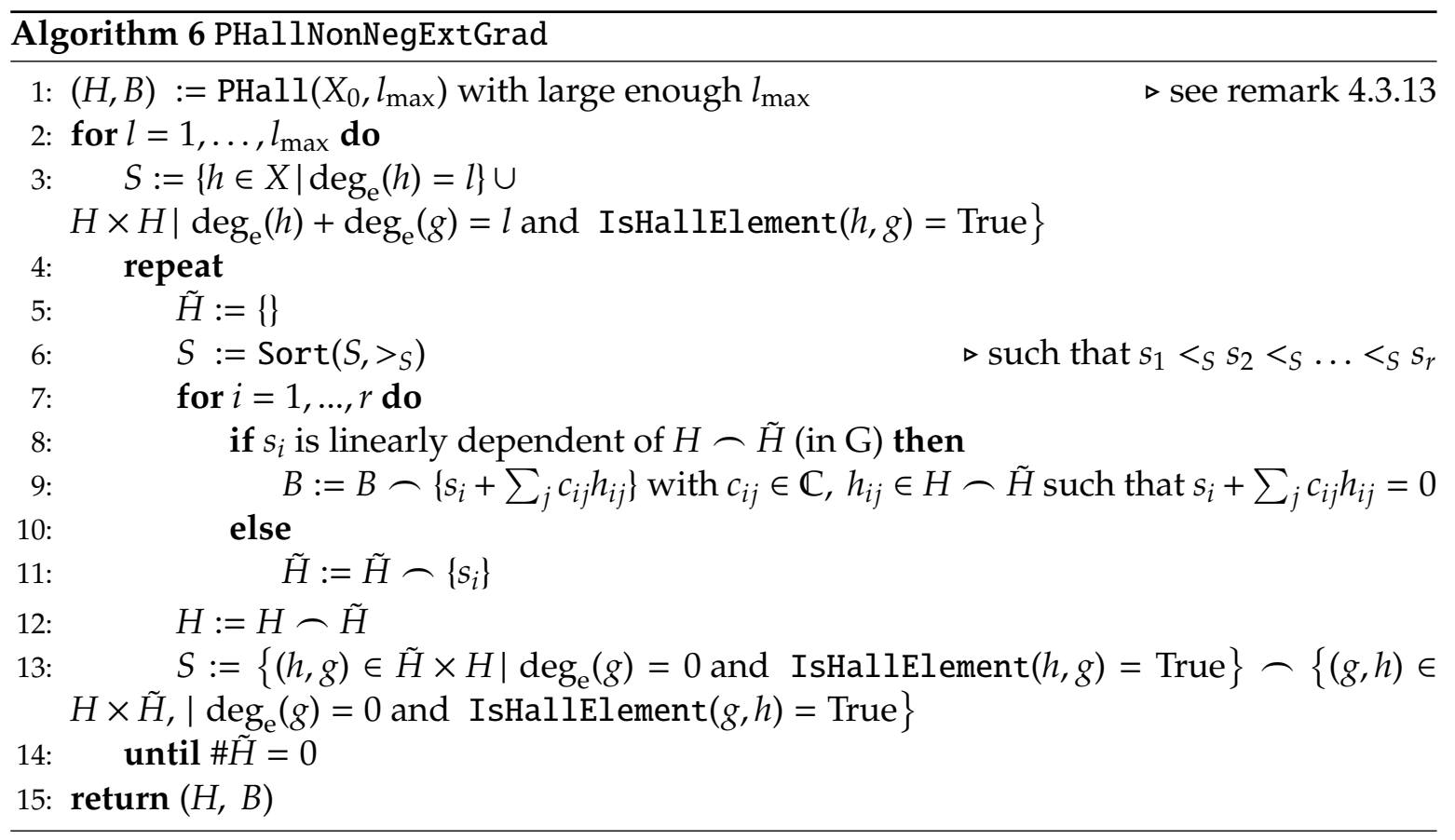

Remark 4.5.1. Since elements of $H$ are linearly independent by construction of the algorithm, the fact that $G_{l}$ is finite-dimensional guarantees that the added repeat . . until loop terminates. The proof of theorem 4.3.3 still applies, so $(H, B)=\operatorname{PHallNonNegExtGrad}\left(X, l_{\max }\right)$ is a pseudo-Hall-basis of $\mathrm{G}$ up to degree $l_{\max }$. 


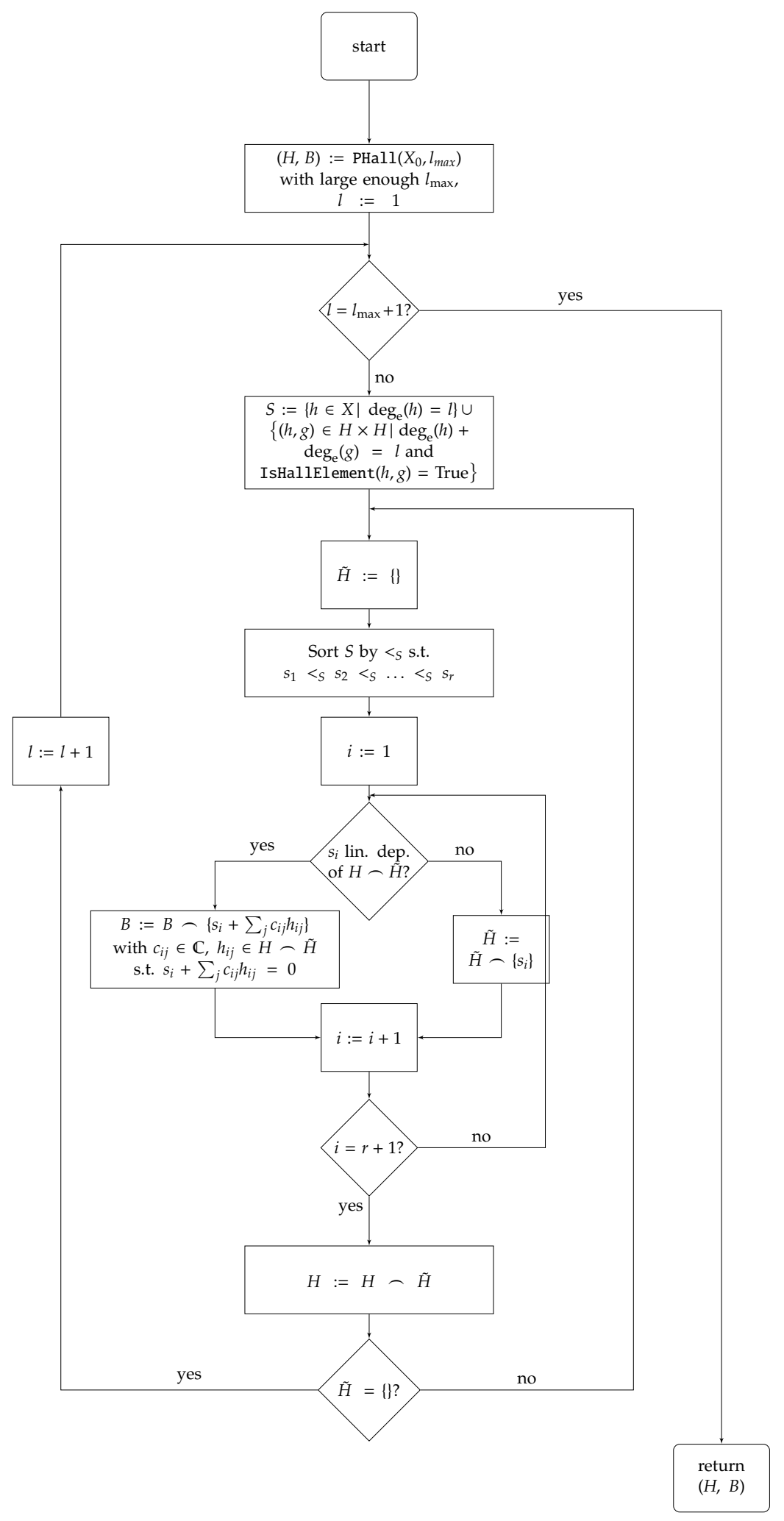

Figure 4.5: Flow chart of algorithm PHallNonNegExtGrad 
Remark 4.5.2. By assumption, $\mathrm{G}_{0}$ is finite-dimensional, and as it is closed under the Lie bracket due to the gradation, it is a finite dimensional Lie algebra, remark 4.3.13 applies.

Remark 4.5.3. For a given external degree $k$, several sets $S$ of candidates for the exension to a basis are computed, and sorting by $>_{S}$ only happens within these sets. So neither $H$ nor $B$ are sorted by $>_{S}$. For instance, for $g \in \mathrm{G}_{k}, h, \tilde{h} \in \mathrm{G}_{0}$, the multiple Lie bracket $[[g, h], \tilde{h}]$ will never be generated as an element of $S$ before $[g, h]$, regardless of $<_{s}$. This can be thought of as losing some control over the order in which elements are considered for the extension of $B$ to a basis. In the last subsection, a way to overcome this problem under stronger preconditions will be discussed.

\section{Using a second gradation}

As it was pointed out in remark 4.3.9, it is useful to partition the vector spaces spanned by the elements of $S$ into subspaces among which no relations exist. Under some preconditions, we have such an opportunity:

1. Let $M$ be a monoid and let the free magma $M(X)$ be equipped with an $M$-gradation $\operatorname{deg}_{\mathrm{M}}$ (in addition to the $\mathbb{N}_{+}$-gradation $\operatorname{deg}_{\mathrm{e}}$ ).

2. Let the gradation $\operatorname{deg}_{M}$ have the property that for each $k \in \mathbb{Z}$, the set

$$
M_{k}:=\left\{m \in M \mid \exists g \in M(X) \text { such that } \operatorname{deg}_{\mathrm{e}} g=k \text { and } \operatorname{deg}_{\mathrm{M}} g=m\right\}
$$
is finite.

3. Let $<_{M}$ be a total order on $M$ with the property that if $g=[a, b]$ with $\operatorname{deg}_{\mathrm{e}} a=0$, then $\operatorname{deg}_{M} b<_{M} \operatorname{deg}_{M} g$.

Now, because of condition 1, if $g, h \in \mathrm{G}$ with $\operatorname{deg}_{\mathrm{M}} g \neq \operatorname{deg}_{\mathrm{M}} h$, then $g$ and $h$ are linearly independent, so we can benefit from partitioning the vector space. The other preconditions allow us to iterate over $M$ with the algorithm PHallSecondGrad found on the next page.

Remark 4.5.4. Condition 2 guarantees that each iteration over $M_{k}$ terminates (and so the entire algorithm does), and condition 3 ensures that each of the possible arguments of the Lie brackets that are the elements of $S$ has been computed and considered for our extension to a basis in a previous step (in a similar way that the positivity of $\operatorname{deg}_{\mathrm{e}}$ was used in remark 4.4.2. 


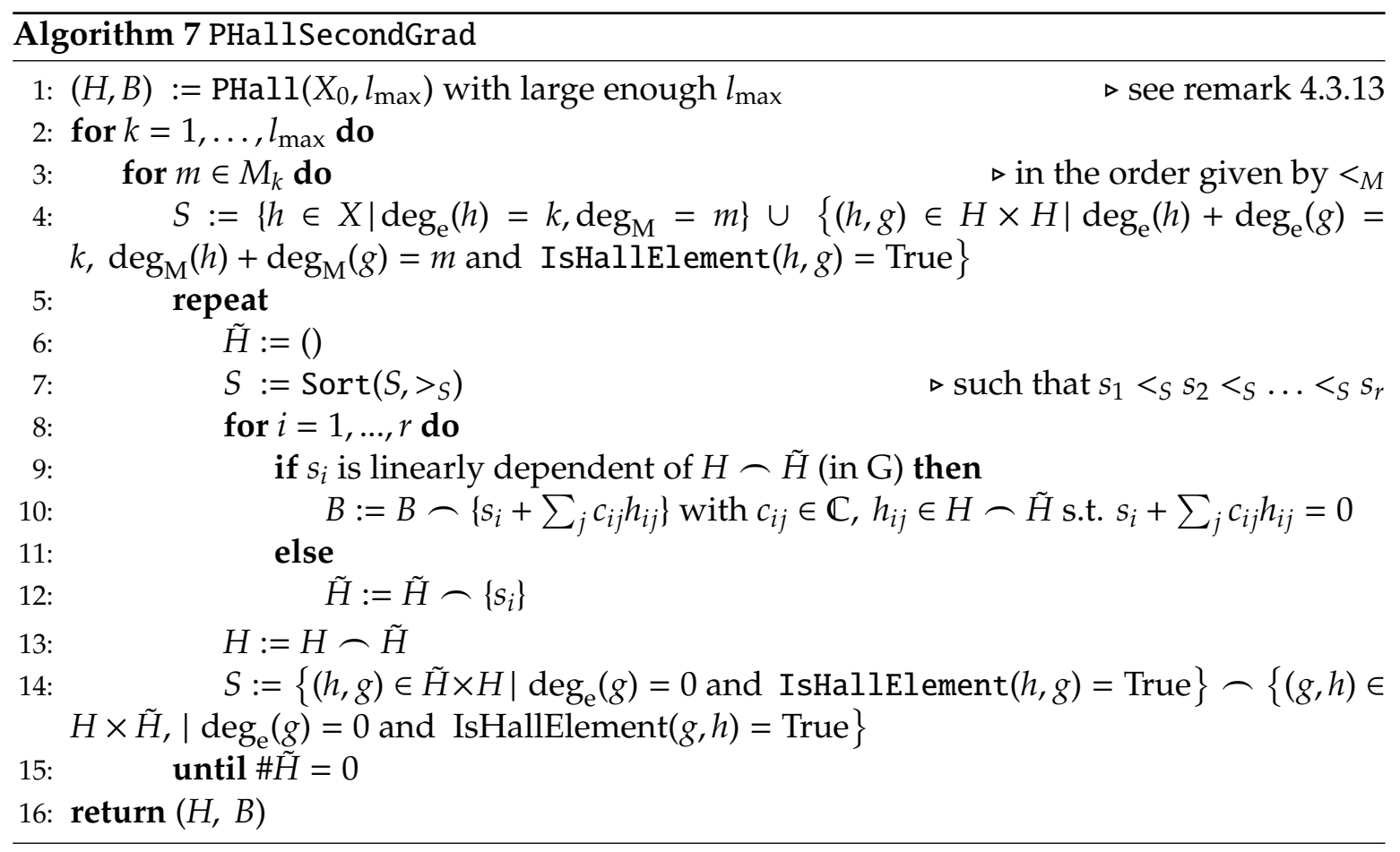

\section{Application to the Pohlmeyer-Rehren Lie algebra}

Remark 4.5.5. 1. In the case of the Pohlmeyer-Rehren Lie algebra, the magnetic quantum numbers provide us with another $\mathbb{Z}^{\left\lfloor\frac{d-1}{2}\right\rfloor}$-gradation denoted by $\operatorname{deg}_{\mathrm{M}}$. The lexicographic order $<_{\text {Lex }}$ on $\mathbb{Z}^{\left\lfloor\frac{d-1}{2}\right\rfloor}$ satisfies condition 3 if we restrict the generating set $X$ to elements that have positive degree and elements of degree zero with a weight tuple $\left(\mu_{i}\right)_{i \in I_{d}}$ satisfying

$$
\mu_{i} \geq 0 \forall i \in I_{d} \quad \text { and } \quad \exists i \in I_{d}: \mu_{i}>0
$$

2. We can restrict $X$ further to a basis of $\mathfrak{g}_{0}$ satisfying equations 4.34 and bases of the (one-dimensional) lowest weight vectors of $\mathfrak{g}_{1}$.

In both cases it is not apparent a priori how large the subalgebra thus generated is; one can immediately conclude from additivity of quantum numbers that $\mathfrak{g}_{-1}$ as well as the subspace of $\mathfrak{g}_{0}$ that does not satisfy equations (4.34) cannot be generated.

Remark 4.5.6 (choices for the Hall order). For the order $<_{H}$ to be a Hall order, it must satisfy $\left(h_{1}, h_{2}\right)>_{H} h_{2}$ for all $h_{1}, h_{2} \in H$. If we restrict $\mathfrak{g}_{0}$ to only those elements $g \in \mathfrak{g}_{0}$ with $\operatorname{deg}_{\mathrm{M}} g>_{D} 0$, a sufficient condition for this is

$$
\begin{aligned}
& a<_{H} b \text { if } \operatorname{deg}_{\mathrm{e}} a<\operatorname{deg}_{\mathrm{e}} b, \\
& a<_{H} b \text { if } \operatorname{deg}_{\mathrm{e}} a=\operatorname{deg}_{\mathrm{e}} b \text { and } \operatorname{deg}_{\mathrm{M}} a<\operatorname{Lex} \operatorname{deg}_{\mathrm{M}} b
\end{aligned}
$$

The Mathematica-Module PHallPMAd3 given in appendix implements the strategy described in this subsection. Its runtime behavior ${ }^{10}$ is the following:

\footnotetext{
${ }^{10}$ All measurements taken with the same Intel Core i7-3770 with 32GiB of RAM running Linux.
} 
- For $d=3$ with $X$ as described in remark 4.5.52 without computing the relations (as lined out in remark 4.3.8):

\begin{tabular}{|l|rrrrrrrr|}
\hline$l$ & 1 & 2 & 3 & 4 & 5 & 6 & 7 & 8 \\
\hline$\# H_{l}$ & 8 & 18 & 48 & 116 & 312 & 810 & 2184 & 5880 \\
$\# B_{l}$ & 2 & 10 & 62 & 186 & 587 & 1873 & 5713 & 16855 \\
$\operatorname{dim} \mathfrak{g}_{l}$ & 8 & 18 & 48 & 116 & 312 & 810 & 2184 & 5880 \\
$t_{\text {comp }}[s]$ & 0.0063 & 0.033 & 0.20 & 1.07 & 10.3 & 192 & $5.1 \mathrm{k}$ & $186 \mathrm{k}$ \\
\hline
\end{tabular}

- For $d=4$, treating $\mathfrak{g}$ as a $\mathfrak{s l}_{2}$-module instead of $\mathfrak{g}_{0} \cong \mathfrak{s l}_{2} \times \mathfrak{s l}_{2}$ (i.e. only using one rather than two raising operators), without computing the relations:

\begin{tabular}{|l|rrrrr|}
\hline$l$ & 1 & 2 & 3 & 4 & 5 \\
\hline$\# H_{l}$ & 20 & 60 & 204 & 670 & 2340 \\
$\# B_{l}$ & 19 & 128 & 574 & 1998 & 8246 \\
$\operatorname{dim} \mathfrak{g}_{l}$ & 20 & 60 & 204 & 670 & 2340 \\
$t_{\text {comp }}[s]$ & 0.052 & 0.38 & 8.13 & 193 & $11.9 \mathrm{k}$ \\
\hline
\end{tabular}

Due to this unusual setup, the relations were not computed in a later step.

- For $d=3$, with full computation of all occurring relations:

\begin{tabular}{|l|rrrrrrrr|}
\hline$l$ & 1 & 2 & 3 & 4 & 5 & 6 & 7 & 8 \\
\hline$\# H_{l}$ & 8 & 18 & 48 & 116 & 312 & 810 & 2184 & 5880 \\
$\# B_{l}$ & 2 & 10 & 69 & 286 & 1061 & 3549 & 11496 & 35584 \\
$t_{\text {comp }}[s]$ & 0.0078 & 0.044 & 0.19 & 1.0 & 25 & 355 & $9.4 \mathrm{k}$ & $413 \mathrm{k}$ \\
\hline
\end{tabular}

The computation was aborted after 11 days and 4 hours of CPU time (approximately $10^{6} \mathrm{~s}$ ) due to memory limitations on a machine equipped with $32 \mathrm{~GB}$ of physical RAM.

Comparing the timing of the computation, one can see a considerable improvement (using the adapted set of generators given above) in performance compared to the basic version PHall of the algorithm.

- For instance, for $d=3$, the computation up to $l=6$ with PHall took more than six hours, compared to less than six minutes.

- For $d=4$, the computation up to $l=4$ took more than 34 hours, while it could be completed in less than a tenth of that time with PHallSecondGrad.

The dimension data confirm that the restricted generated sets described above in 4.5 .5 actually generate $\mathfrak{g}_{\geq 1} \oplus\left(\mathfrak{g}_{0} \cap X\right)$ up to the limit of computation. In particular, it follows that $\mathfrak{g}_{0} \oplus \mathfrak{g}_{1}$ generate $\mathfrak{g}_{\geq 0}$ up to the limit of computation, lending credence to conjecture 4.1.3.

Remark 4.5.7. The algorithm uses the facts that weights (here: magnetic quantum numbers) are additive under the Lie bracket. The highest weights (here: spin tuples) that determine (up to equivalence) the multiplets do not behave as simply however. Even if generators are contained in a single multiplet, in general Hall words of these generators are not contained in a single multiplet as well, but rather in a direct sum of several multiplets (determined by Clebsch-Gordan formulas such as theorem 2.4.2). 
It is an interesting approach to find linear bases of the strata $\mathfrak{g}_{l}$ of elements that do not only have well-defined weight (magnetic quantum number tuple), but highest weight (spin tuple) as well by modifying the algorithm further such that instead of a Hall word $h \in \mathfrak{g}_{l}^{m}=\bigoplus^{s} \mathfrak{g}_{l}^{m}$ (where the values of $s$ in the direct sum are controlled by the Clebsch-Gordan formula), a projection of $h$ on the space ${ }^{s} \mathfrak{g}_{l}^{m}$ for an appropriate highest weight $s$ is used.

There are two problems with this approach. The first is to make sure that the selection of projected Hall words have the same span as the Hall words in a given stratum. This can be achieved by starting with the highest highest weights in the stratum. One can then iteratively consider lower highest weights.

The second problem is: while we have a guarantee that the projections of the Hall words mentioned above from the $l$-th stratum span that $l$-th stratum, no such guarantee exists for higher $(>l)$ strata because the projections are not Hall words. It is an interesting question if the theory of Hall bases can be modified in such a way that projections of Hall words onto irreducible $\mathfrak{g}_{0}$-modules play a similar role as Hall words do in free Lie algebras while simultaneously respecting the decompositions into irreducible $\mathfrak{g}_{0}$-modules and weight spaces as they were explored in chapter 2 of this thesis.

\subsubsection{Iterative generation and subsequent extension to a basis}

The approach given in 4.5.1 has the disadvantage that the sequence in which elements of $S$ were considered for extension to a basis of $\mathrm{G}_{k}$ could not be choosen freely. If we can iteratively generate all candidates for extension to a basis first and only then extend to a basis, we can avoid this problem. We will see that imposing the following additional conditions on the Lie algebra $\mathrm{G}$ allows to avoid cases where the algorithm never terminates.

Definition 4.5.8 (pseudo-Hall-exhaustibility). A Lie algebra G equipped with a $\mathbb{N}_{0}$-gradation $\operatorname{deg}_{\mathrm{e}}$ with the properties

1. the dimensions $\operatorname{dim} G_{l}$ are finite for all $l$ ( $G$ is degreewise finite dimensional),

2. for all $l$ exists $n_{l} \in \mathbb{N}_{0}$ such that

$$
\operatorname{ad}_{g_{n_{l}}} \operatorname{ad}_{g_{n_{l}-1}} \ldots \operatorname{ad}_{g_{1}} g=0 \quad \forall g_{i} \in \mathrm{G}_{0}, g \in \mathrm{G}_{l}
$$

is called pseudo-Hall-exhaustible.

Remark 4.5.9. In particular, this obviously implies that $\mathrm{G}_{0}$ is a finite-dimensional subalgebra.

Example 4.5.10. The following Lie algebras are pseudo-Hall-exhaustible:

1. finite-dimensional nilpotent Lie algebras with the trivial gradation; here $G=G_{0}$,

2. finitely generated Lie algebras that are graded by the monomial degree; here $\mathrm{G}_{0}=0$, $\mathrm{G}_{1}$ is spanned by the generators,

3. $\left\langle e(0+), \mathfrak{g}_{l>0}\right\rangle \subset \mathfrak{g}$ (the Pohlmeyer-Rehren Lie algebra in $d=3$ ).

The last example is a special case of the following more general proposition. 
Proposition 4.5.11. Let $\mathrm{G}$ be a degreewise finite-dimensional $\mathbb{N}_{0}$-graded Lie algebra with semisimple stratum $\mathrm{G}_{0}$.

Recall that in this situation (cf. subsection 2.2.3) $\mathrm{G}_{0}$ has a root space decomposition with base $\left\{\alpha_{1}, \ldots, \alpha_{b}\right\}$ and set of positive roots $R^{+}$, and (cf. subsection 2.3.1) each stratum $\mathrm{G}_{l}$ can be decomposed into weight spaces relative to $\mathrm{G}_{0}$.

Then the Lie subalgebra $\tilde{\mathrm{G}}$ (of $\mathrm{G}$ ) generated by

$$
\left\{x \in \mathrm{G}_{0}^{\beta} \mid \beta \in R^{+}\right\} \cup \mathrm{G}_{\geq 1}
$$

is pseudo-Hall-exhaustible.

Proof. Consider that for $l>1, \tilde{\mathrm{G}}_{l}=\mathrm{G}_{l}$ is a $\mathrm{G}_{0}$-module, so the same weight space decomposition applies. Without loss of generality (otherwise, decompose $g$ and $g_{i}$ accordingly) $g \in \mathrm{G}_{l}^{\lambda}$ for some weight $\lambda \in \Gamma$ and $g_{i} \in g_{0}^{\beta_{i}}$ for some positive $\operatorname{root} \beta_{i} \in R^{+}$for all $i$. By theorem 2.3.32 there exist $k_{i} \in \mathbb{N}_{0}, i \in\{1, \ldots, b\}$ such that

$$
\lambda=\sigma_{l}-\sum_{i=1}^{b} k_{i} \alpha_{i},
$$

where $\sigma_{l}$ is the highest weight of $\mathrm{G}_{l}$. In particular,

$$
K:=\sum_{i=1}^{n} k_{i} \geq 0 .
$$

Similarly, by the definition of $R^{+}$, there exist coefficients $m_{i}^{j} \in \mathbb{N}_{0}$ such that for all $\beta^{j} \in R^{+}$:

$$
\beta^{j}=\sum_{i=1}^{n} m_{i}^{j} \alpha_{i}
$$

with $\sum_{i=1}^{n} m_{j}^{i}>0$ (otherwise, $\beta_{j}=0 \notin R^{+}$). Now, because of theorem 2.3.2 2

$$
\operatorname{ad}_{g_{p}} \operatorname{ad}_{g_{p-1}} \ldots \operatorname{ad}_{g_{1}} g \in \tilde{G}_{l}^{\lambda_{p}}
$$

with

$$
\lambda_{p}:=\lambda-\overbrace{\left(k_{i}-\sum_{j=1}^{p} m_{i}^{j}\right)}^{=: k_{i}^{j}} \alpha_{i} .
$$

But for $p>K$ we have

$$
K^{p}:=\sum_{i=1}^{n} k_{i}^{p}<0,
$$

so $\mathrm{G}_{l}^{\lambda_{p}}=0\left(\lambda_{p}\right.$ is not a weight $)$ because otherwise, there would be a contradiction to the analogue to equation (4.40) for $\lambda_{p}$ (instead of $\lambda$ ) that would hold if $\lambda_{p}$ were a weight. 
Remark 4.5.12. Pseudo-Hall-exhaustibility is a stronger property for graded Lie algebras than being degreewise finite dimensional. For instance, the Pohlmeyer-Rehren Lie algebra for $d=3$ is degreewise finite dimensional but not pseudo-Hall-exhaustible, because G can be decomposed into eigenspaces of $\mathrm{ad}_{h}$, an inner automorphism that is not identically zero.

Let now $\mathrm{G}$ be a pseudo-Hall-exhaustible Lie algebra generated by the degreewise finite set $X$. In this context, we can formulate the following variation of PHall:

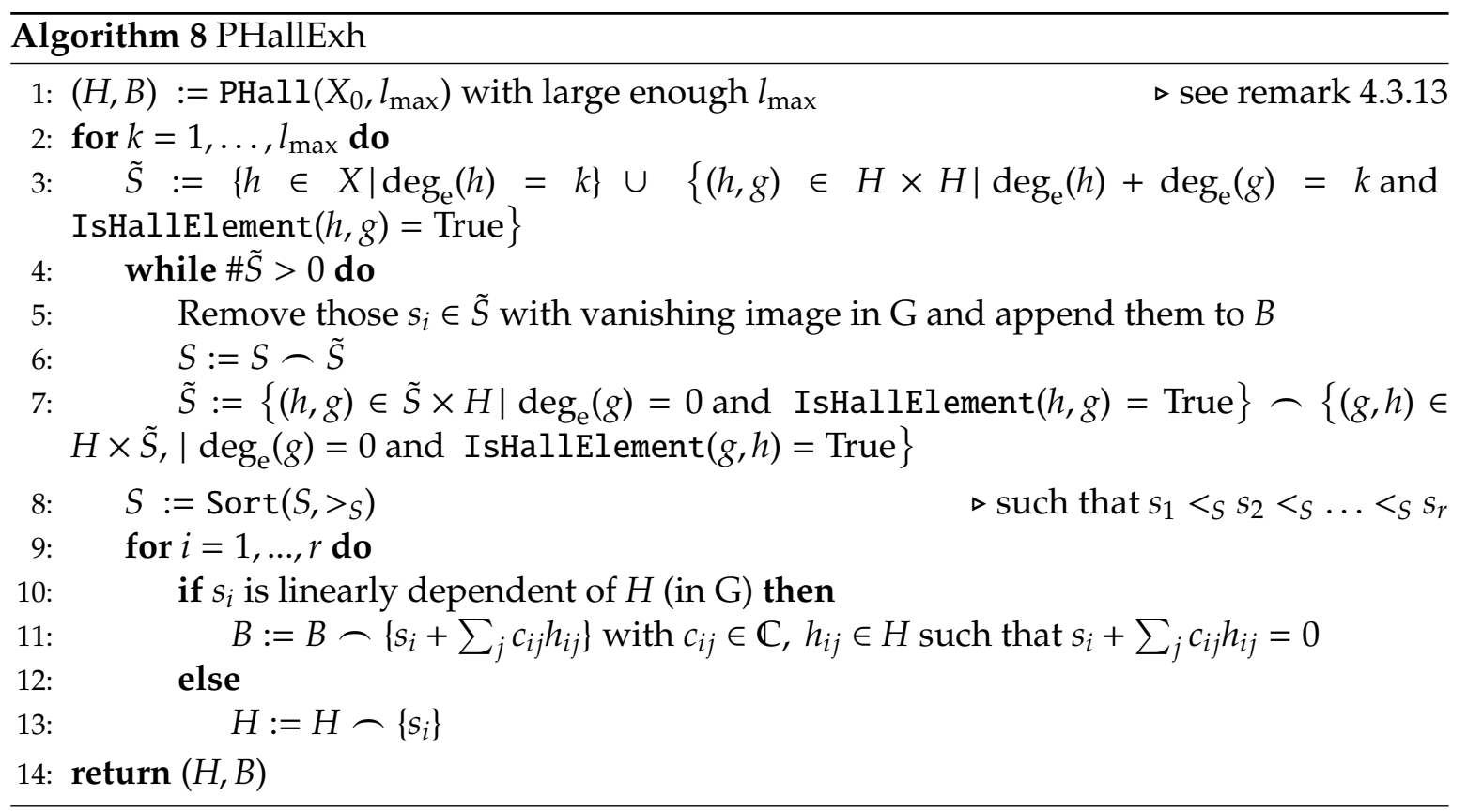

Remark 4.5.13. Since each iteration of the while loop in step 4 consists of adjoining elements of external degree zero, condition 3 of pseudo-Hall-exhaustability implies that after the $n_{k}+1$ th iteration of the loop, $\tilde{S}=\{\}$, so the exit condition of the while loop is met, the loop is finite and the algorithm terminates. 


\section{Chapter 5}

\section{Outlook}

\subsection{Interplay of the Hall algorithm and representation theory}

Throughout the entire chapter 4 , we have always used Hall orders $<_{H}$ and sorting orders $<_{S}$ that were given a priori. It might be worthwhile to go the other way around and construct Hall and sorting orders that are tailored to the problem at hand.

Of particular interest might be Hall and sorting orders that give us a shortest pseudoHall-basis by minimizing $\# H_{k}$.

In situations where some of the representation theory of the Lie subalgebra $G_{0}$ is already known (for instance, $\mathfrak{g}_{0} \cong \mathfrak{s} \mathfrak{v}(d, \mathbb{C})$ in the case of the Pohlmeyer-Rehren Lie algebra that will be considered in the sequel), in the spirit of remark 4.5.5 we might want to find a set $H$ that consists - as far as possible given the constraints, for instance imposed by the axioms for Hall sets - of lowest weight vectors of irreducible $\mathfrak{g}_{0}$-modules (expressed in terms of brackets of elements of lower degree) and their multiple adjoints of elements of $\mathfrak{g}_{0}$ satisfying equation (4.34). This would allow us to see the $\mathfrak{g}_{0}$-modules more clearly in the pseudo-Hall-basis.

Unfortunately, it isn't immediately clear how to approach this problem, and it also isn't clear how successful we can hope to be.

Example 5.1.1. Consider the situation of remark 4.5.6 for $d=3$ and further suppose that we have found some Hall element $v_{0}=\left[a_{0}, v_{1}\right] \in \mathfrak{g}_{l}^{m} .\left(v_{0} \in \mathfrak{g}_{l}^{m}\right.$ with some $m, l$ automatically follows if the elements of $X$ have definite degree and magnetic quantum number.) Now, the condition

$$
v \in H \Rightarrow \operatorname{ad}_{x}^{n} v \in H \text { for all } n \in \mathbb{N}_{0}
$$

would be desirable because this aligns the Hall set neatly with the irreducible $\mathfrak{g}_{0}$-modules. But then, in particular $\left[x, v_{0}\right] \in H$. By axiom $2(\mathrm{~b})$ of a Hall set $(4.2 .6)$, this implies $v_{0} \in X$ or $a_{0} \leq_{H} x$. In the former case, $v_{0} \in \mathfrak{g}_{l} \cap X$. We consider the latter case.

Now suppose the conditions from 4.5 .6 on $<_{H}$ are met, then this implies $a_{0}=x$, and therefore further $v_{1} \in \mathfrak{g}_{l}^{m-1}$ ). Since we have $a_{0}=x$, we can inductively apply the reasoning applied to $v_{0}$ to $v_{1}$ and inductively further to $v_{i}$ (that have the property $v_{0}=\operatorname{ad}_{x}^{i} v_{i}$ ). Because we cannot continue the iteration more than $m+l+1$ times before the latter case is excluded due to corollary 2.3.12,3, it follows that

$$
v_{0}=\operatorname{ad}_{x}^{r} v_{r}
$$


with some $v_{r} \in X$ with $r \leq m+l+1$ and $\operatorname{deg}_{\mathrm{e}} v_{r}=\operatorname{deg}_{\mathrm{e}} v_{0}$, in particular $v_{r} \in \mathfrak{g}_{l}^{m-r}$.

We have shown that we can't express the elements $v_{r}$ as brackets of elements of lower degree, unlike our intention. Since this can be done for arbitrarily high $l$, the set $X$ must be infinite.

If we want to uphold condition (5.1), we therefore have to choose a Hall order $<_{H}$ that violates the conditions from 4.5.6. But unfortunately, it is not obvious how to do this.

\subsection{Open questions regarding the Pohlmeyer-Rehren Lie algebra}

While we have found a way to understand the Pohlmeyer-Rehren Lie algebra in terms of its multiplets, a clear understanding of its multiplicative structure is still a desideratum. The application of the Hall algorithm to the problem has lead to some insights, but as we have seen in the above example 5.1.1. the questions raised at the end of section 2.4 about the origin of the relations in the context of the Clebsch-Gordan problem are not easily solved.

Furthermore, the conjectures about the (finite) generation of $\mathfrak{g}$ in terms of its strata $\mathfrak{g}_{l}$ with $l \leq 1$ from section 4.1 are still lacking a proof or disproof. Of particular interest would be a pseudo-Hall-basis with a closed expression for all the relations.

It is an interesting fact that Hall words play a dual role in the available description of the Pohlmeyer-Rehren Lie algebra g. First, because it is a Lie algebra, and as such isomorphic to a free Lie algebra modulo an ideal (theorem 4.2.4), a basis of which is given by the Hall words of its generators (theorem 4.2.12). Second, and more specific to $\mathfrak{g}$, because the Euler-Lyndon words that are a basis of $\mathfrak{g}$ contain Hall words (theorem 4.2.9). The question if this can be exploited in some form suggests itself. A negative partial result was found in proposition 4.2.11 (with the very simple assumption that the ideal be zero), but so far, no serious attempt to leverage this in the general case has been made. 


\section{Appendix A}

\section{Mathematica Code for Pseudo-Hall-Bases}

In this appendix, Mathematica code implementing the algorithms introduced in chapter 4 is given. All code is written for Mathematica 9.0.1.0, and all computations were performed using that version of the software.

\section{A.1 Orders}

\section{A.1.1 LettOrd}

Lett0rd implements the order of individual letters underlying both DegLex and Lex. Input are two letters $a, b \in X$, output is, using the notation of the built-in function Order,

$$
\begin{array}{r}
-1 \text { if } a<b, \\
0 \text { if } a=b, \\
+1 \text { if } a>b .
\end{array}
$$

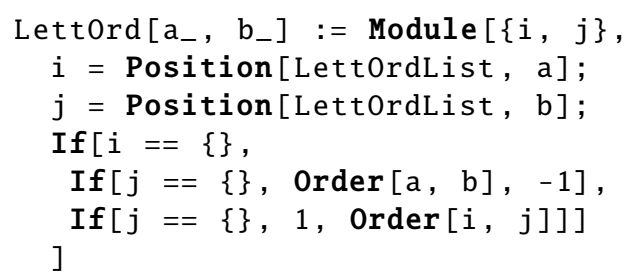

LettOrd depends on a global variable LettOrdList to be set which is a list of letters in ascending order, for instance

LettOrdList $=\{" x ", " v ", " w "\}$

encodes the order of letters $x<v<w$.

\section{A.1.2 Lex}

Lex implements the lexicographic order (definition 1.3.11) on $M(X)$. Input is two elements $\mathrm{A}, \mathrm{B} \in M(X)$, output uses the same notation as Lett0rd. For the input, both elements of $M(X)$ 
denoted by curly brackets and elements of $L(X)$, the Lie bracket denoted by $K[\ldots, \ldots]$ are accepted.

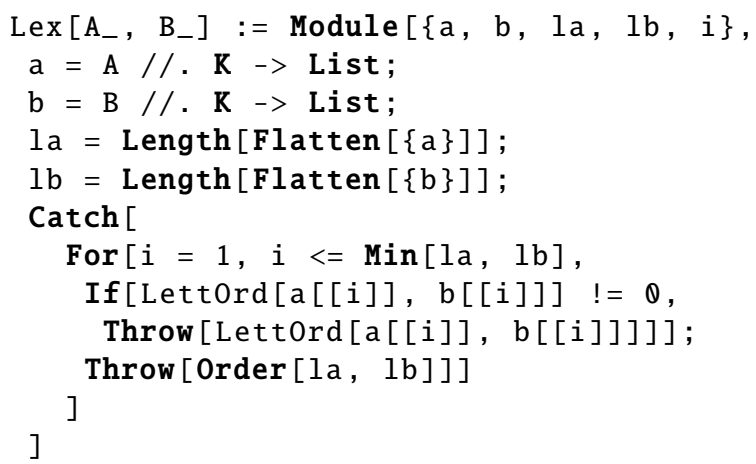

For the required comparisons of individual letters, the module Lett0rd is called.

\section{A.1.3 DegLex}

DegLex implements the Hall order of the same name (definition 1.3.12) on $M(X)$. Input is two elements $\mathrm{A}, \mathrm{B} \in M(X)$ as above, output uses the same notation as Lett0rd again. For all examples in the sequel, we will set Hallord := DegLex.

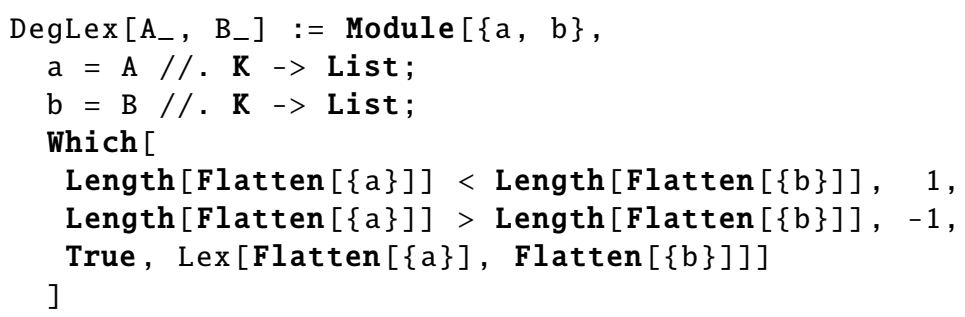

If the lengths of the elements compared are equal, the lexicographic order Lex is called.

\section{A.2 Hall's original algorithm}

\section{A.2.1 IsHallElement}

IsHallElement implements the homonymous algorithm from section 4.2 . Input is a list $\mathrm{h}$ of two (possibly nested) lists represending elements of $M(X)$, output is a boolean. Note that IsHallElement is only used for compositions of Hall elements, so the check if their constituents are actually Hall elements is commented out.

A Hall set, and with it the output of IsHallElement, depends on the Hall order. Since the Hall order does not change during a computation, this dependence is handled implicitly (as opposed to explicitly by passing a Hall order as an argument) by calling the module Hallorder.

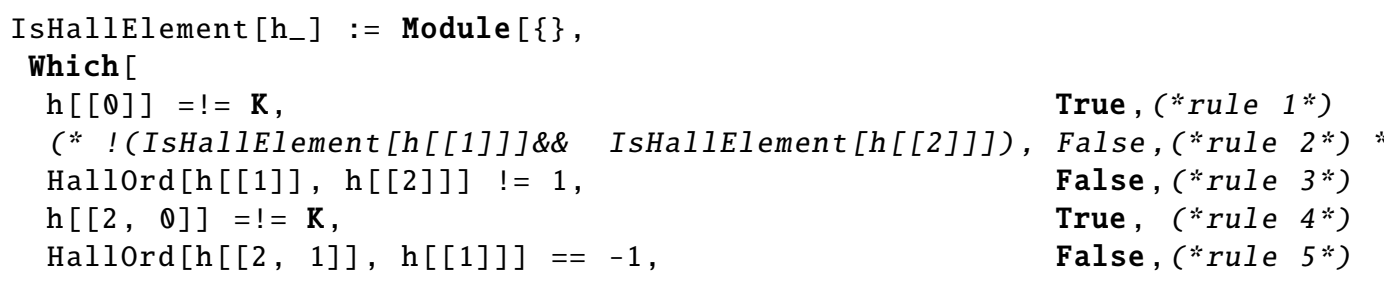


True ,

True (*rule 6*) ] ]

Example A.2.1. IsHallElement [\{"2", \{"1", "2" $\}\}]$ yields True, while IsHallElement [\{"1", "1"\}] yields False.

\section{A.2.2 Hall}

Hall implements HaLL's algorithm (see section 4.2). Input is a list of generators $\mathrm{X}$ and a maximum degree to compute up to $\mathrm{d}$, output is the List $\mathrm{H}$ of elements, each given in the form $\left\{\mathrm{x} \in M(X), \operatorname{deg}_{H} \mathrm{x}\right\}$.

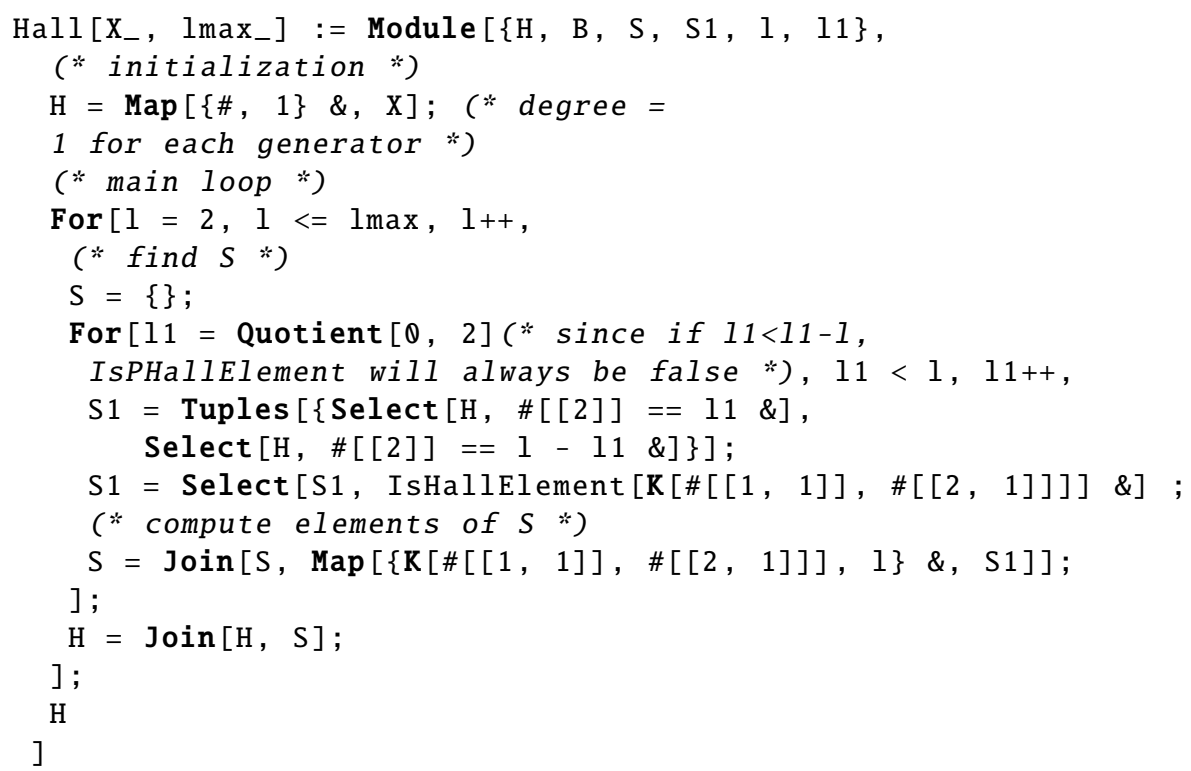

Example A.2.2. $\operatorname{Hall}[\{" \mathrm{x} ", \mathrm{y} "\}, 5] / /$.K- - List// Grid

(the replacement //.K->List is just added to improve readability) yields

$\begin{array}{lr}\mathrm{x} & 1 \\ y & 1 \\ \{\mathrm{x}, \mathrm{y}\} & 2 \\ \{\mathrm{x},\{\mathrm{x}, \mathrm{y}\}\} & 3 \\ \{\mathrm{y},\{\mathrm{x}, \mathrm{y}\}\} & 3 \\ \{\mathrm{x},\{\mathrm{x},\{\mathrm{x}, \mathrm{y}\}\}\} & 4 \\ \{\mathrm{y},\{\mathrm{x},\{\mathrm{x}, \mathrm{y}\}\}\} & 4 \\ \{\mathrm{y},\{\mathrm{y},\{\mathrm{x}, \mathrm{y}\}\}\} & 4 \\ \{\mathrm{x},\{\mathrm{x},\{\mathrm{x},\{\mathrm{x}, \mathrm{y}\}\}\}\} & 5 \\ \{\mathrm{y},\{\mathrm{x},\{\mathrm{x},\{\mathrm{x}, \mathrm{y}\}\}\}\} & 5 \\ \{\mathrm{y},\{\mathrm{y},\{\mathrm{x},\{\mathrm{x}, \mathrm{y}\}\}\}\} & 5 \\ \{\mathrm{y},\{\mathrm{y},\{\mathrm{y},\{\mathrm{x}, \mathrm{y}\}\}\}\} & 5 \\ \{\{\mathrm{x}, \mathrm{y}\},\{\mathrm{x},\{\mathrm{x}, \mathrm{y}\}\}\} & 5 \\ \{\{\mathrm{x}, \mathrm{y}\},\{\mathrm{y},\{\mathrm{x}, \mathrm{y}\}\}\} & 5\end{array}$




\section{A.3 Variants of the Hall algorithm}

\section{A.3.1 PHall}

PHall implements the algorithm from section 4.3. Input is a list of generators $\mathrm{X}$, each given as a symbol $\mathrm{x}$ and a corresponding internal representation to be used in computations $\mathrm{rep}[\mathrm{x}]$, as well as a maximum degree to compute up to lmax.

Output is the pair $\{\mathrm{H}, \mathrm{B}\}$ consisting of the list $\mathrm{H}$ of elements of $H$, each given in the form $\left\{\mathrm{x} \in M(X), \operatorname{deg}_{H} \mathrm{x}, \operatorname{rep}[\mathrm{x}]\right\}$, and the list $\mathrm{B}$ of elements of the form $\{\mathrm{LM}[\mathrm{x}], \mathrm{x}-\operatorname{LM}[\mathrm{x}]$, $\left.\operatorname{deg}_{\mathrm{H}} \mathrm{LM}[\mathrm{x}]\right\}$ (note that $\mathrm{rep}[\mathrm{b}]=0$ for $\mathrm{b} \in \mathrm{B}$ by construction). PHall uses the function PHallDim, which gives the dimension of $G_{l}$. For instance, in the case of the PohlmeyerRehren Lie algebra for $d=3$, we can use

PHallDim [1_] = NumLyndon $[1+2,3]$

If the dimension of $G_{l}$ is not known, one can instead set

PHallDim[1_] = Infinity

To actually consider the elements of $\mathrm{S}$ for inclusion into the lists $\mathrm{H}$ and $\mathrm{B}$, the module AppendPHB (see A.3.2) is called. Note that if the argument PHallDim[1] is less than $\operatorname{dim} G_{l}$, the linear equations considered in the module AppendPHB have no solution, which will lead to errors. In the computation of $\mathrm{S}$, the module $\mathrm{LB}$, calculating the Lie bracket of two elements of $G$, is called, which has to be defined accordingly.

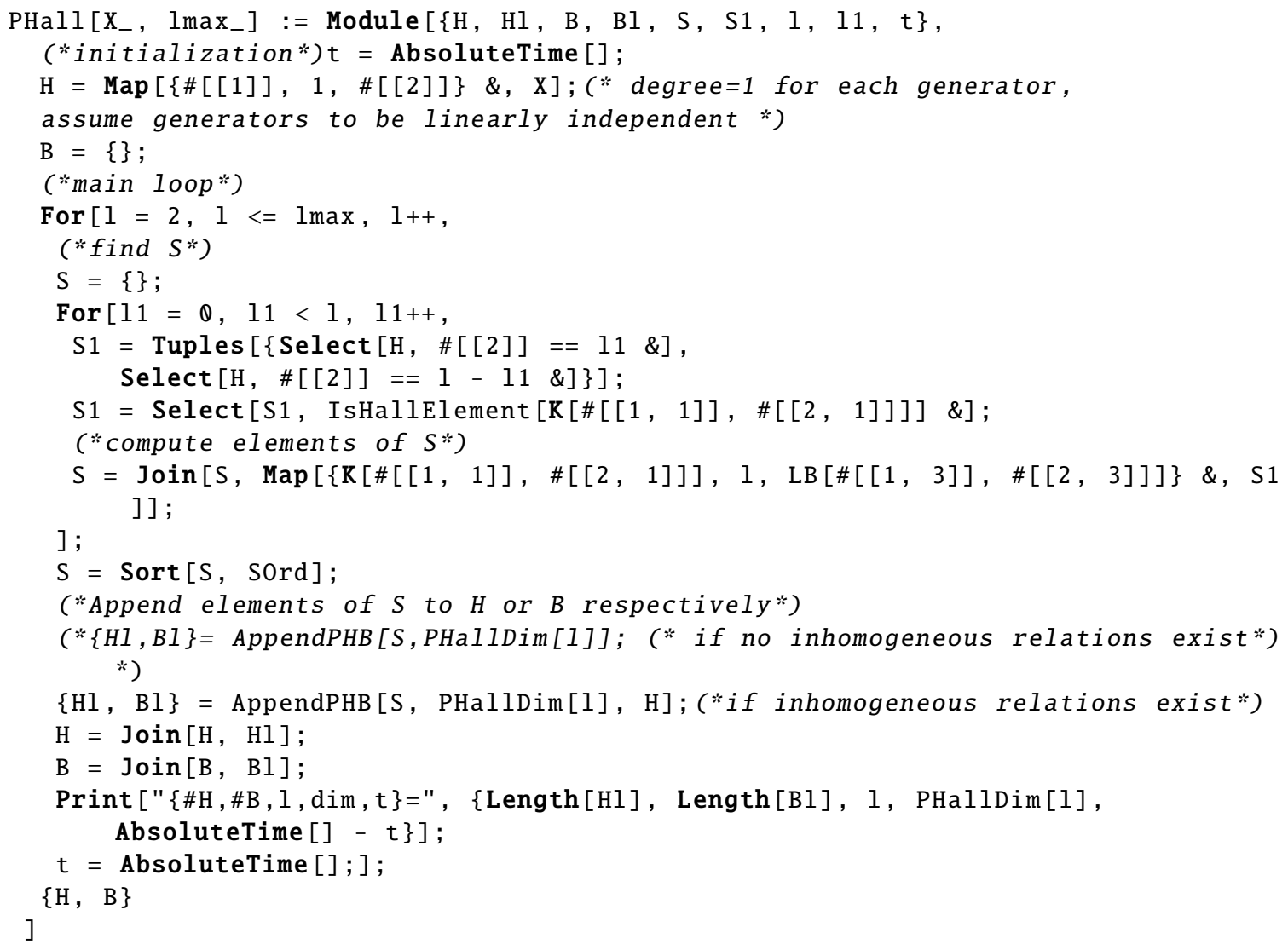

Example A.3.1. Regardless if we choose not to use the known dimension by setting 
PHallDim[1_] = Infinity

or set the more specific

PHallDim $\left[1_{-}\right]=3$

if we use the code for existing inhomogeneous relations commented out in the listing above, in accordance with example 4.3.12, the command

PHall $[\{\{" x ", \quad r[\{\theta, P\}]\},\{" y ", \quad r[\{\theta, M\}]\},\{" h ", \quad r[\{M, P\}]\}\}, 10] / / . K->$ List

yields as result for $B$

$\begin{array}{lll}\mathrm{x} & 1 & \mathrm{r}[\{\theta, \mathrm{P}\}] \\ \mathrm{y} & 1 & \mathrm{r}[\{\theta, \mathrm{M}\}] \\ \mathrm{h} & 1 & \mathrm{r}[\{\mathrm{M}, \mathrm{P}\}]\end{array}$

and as result for $\mathrm{H}$

$\{\mathrm{y}, \mathrm{x}\} \quad-2 \mathrm{~h} \quad 2$

$\{\mathrm{h}, \mathrm{x}\} \quad 2 \mathrm{x} \quad 2$

$\{\mathrm{h}, \mathrm{y}\} \quad-2$ y $\quad 2$ 


\section{A.3.2 AppendPHB}

AppendPHB implements the extension to a basis in the loop starting in step 6 of PHall (and other versions of the Philip Hall algorithm for non-free Lie algebras), iteratively building a list $\mathrm{Hl}$ of elements that are linearly independent among themselves and from the list SOld of elements of $M(X)$, and a list $\mathrm{Bl}$ of relations by iteratively checking the list entries of SNew for linear independence.

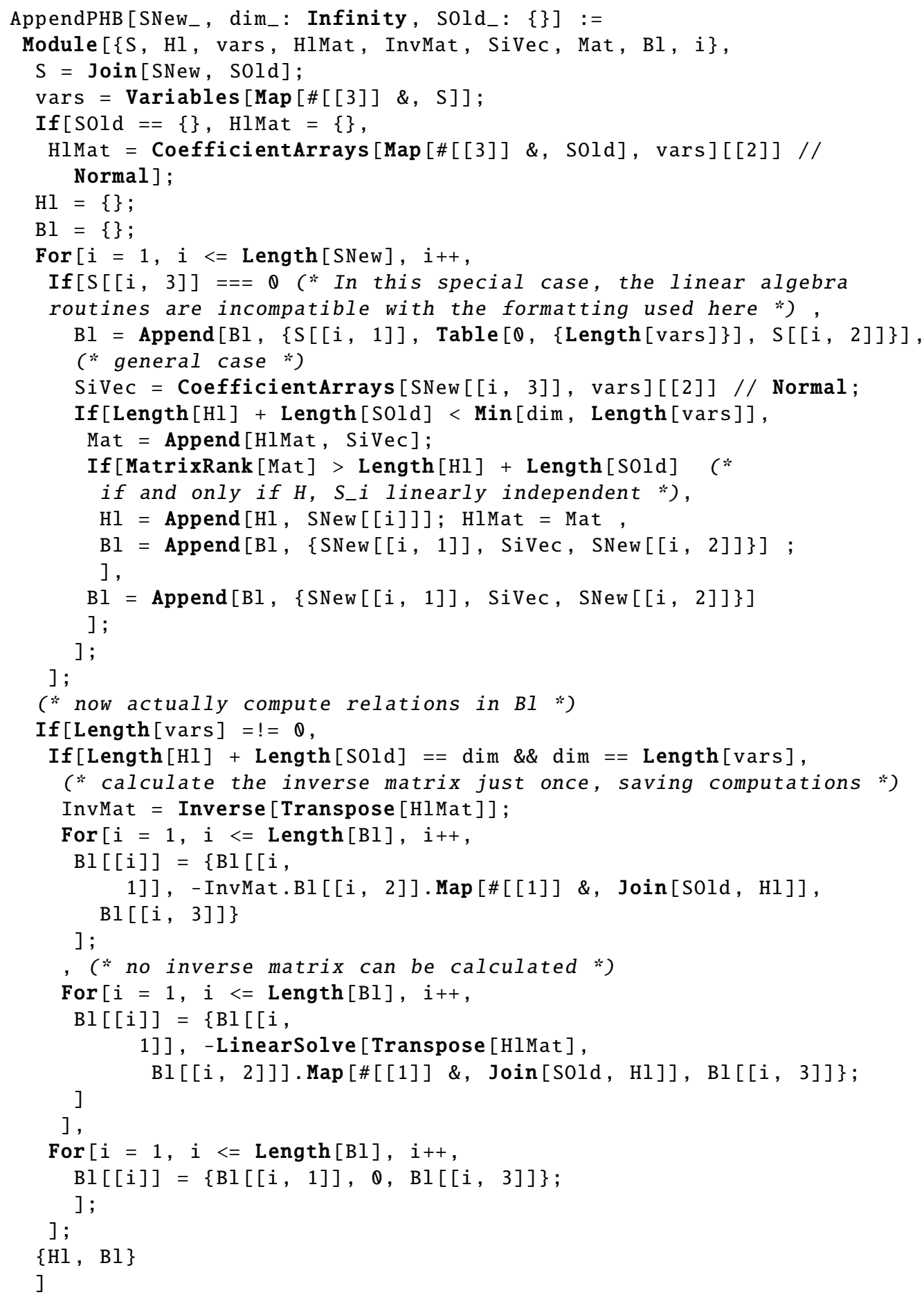


Note that if a formula for $\operatorname{dim} G_{l}$ is available (such as theorem 1.3.6 for the PohlmeyerRehren Lie algebra), a check if Length $[\mathrm{Hl}]>\operatorname{dim} G_{l}$ is used to skip the calculation of linear independence using MatrixRank [Mat]. This is why the argument dim is passed to AppendPHB. Its default value is set to Infini ty because at this value, the condition for skipping calculating the matrix rank never applies.

\section{A.3.3 PHallPosExtGrad}

PHallPosExtGrad implements the algorithm from section 4.4. The input is a list of generators $\mathrm{X}$ of the form $\left\{\mathrm{x}, \operatorname{deg}_{\mathrm{e}} \mathrm{x}, \operatorname{rep}[\mathrm{x}]\right\}$, and an integer lmax giving the degree to compute up to. The output is, like for the module PHall, a list $H$ of the form $\left\{x, \operatorname{deg}_{\mathrm{e}} \mathrm{x}, \operatorname{rep}[\mathrm{x}]\right\}$ and $\mathrm{a}$ list $B$ of the form $\left\{\operatorname{LM}[\mathrm{x}], \mathrm{x}-\mathrm{LM}[\mathrm{x}], \operatorname{deg}_{\mathrm{e}} \mathrm{LM}[\mathrm{x}]\right\}$.

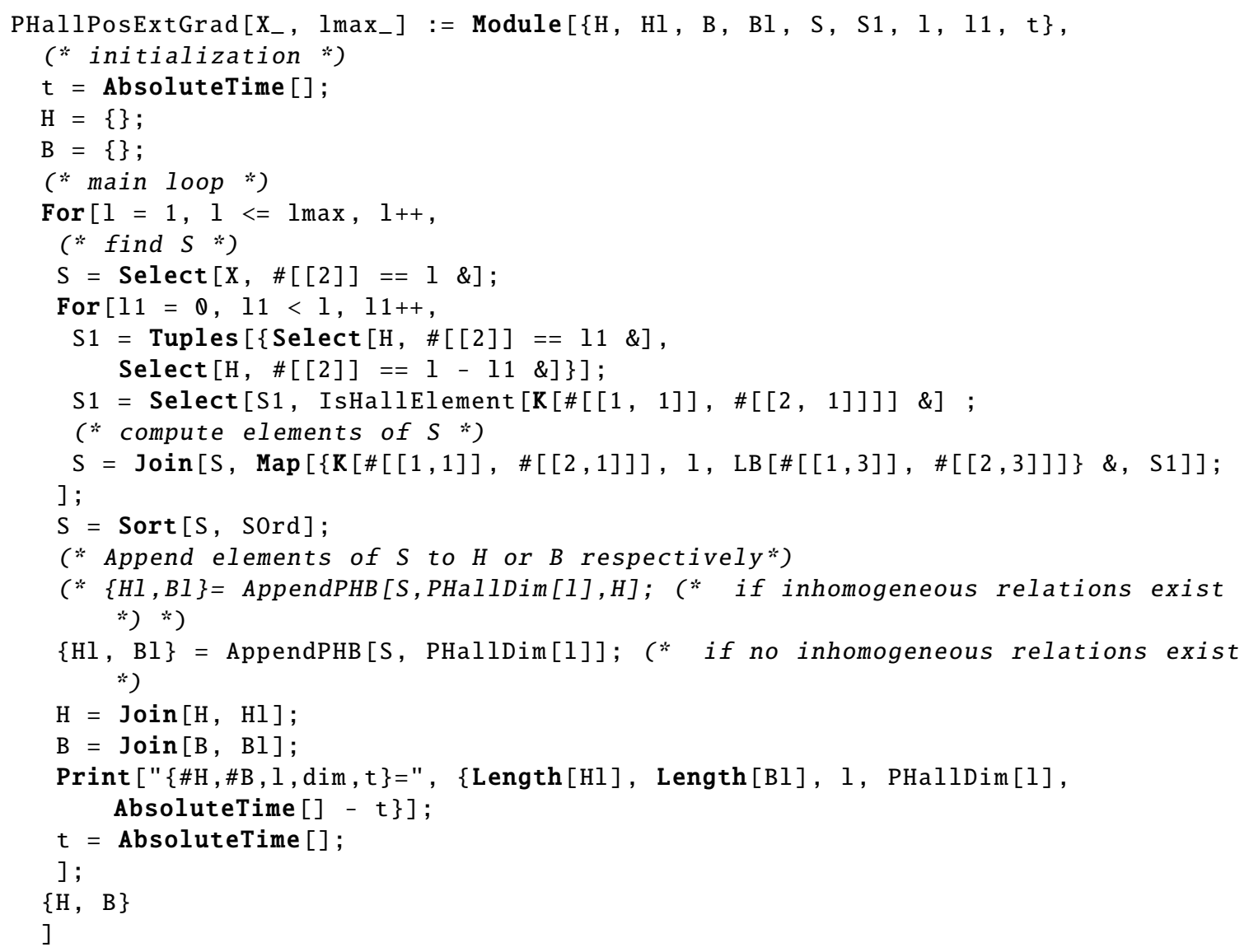




\section{A.3.4 PHallSecondGrad}

PHallSecondGrad is an implementation of the algorithm of the same name given in subsection 4.5.1. Here, the second gradation used is another $\mathbb{Z}$-gradation denoted by $m \in \mathbb{Z}$ with the additional property that $-l-1 \leq m \leq l+1$, such as the magnetic quantum number of the Pohlmeyer-Rehren Lie algebra for $d=3$. Input is a list $\mathrm{X}$ of generators of $G$ of the form $\left\{x, \operatorname{deg}_{e} x, \operatorname{rep}[x], \operatorname{deg}_{M}[x]\right\}$, output are a list $H$ of the same format and a list $B$ of the form $\left\{\operatorname{deg}_{e} \operatorname{LM}[x], \operatorname{deg}_{M} \operatorname{LM}[x], \operatorname{LM}[x], x-\operatorname{LM}[x]\right\}$.

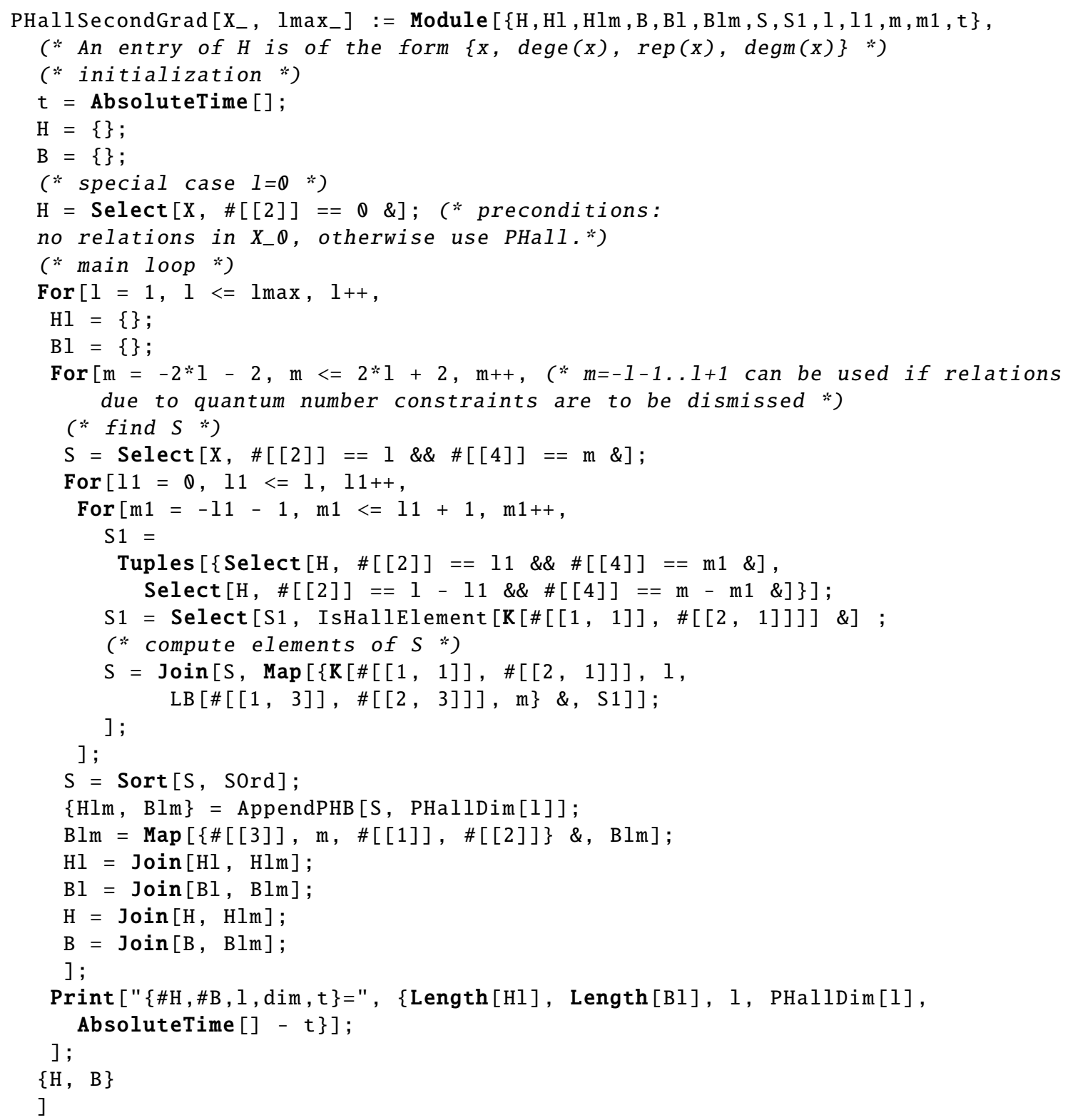




\section{A.3.5 PHallNonNegExtGrad}

The module PHallNonNegExtGrad implements the algorithm of the same name in 4.5.2. The input and output conventions are the same as for PHallPosExtGrad.

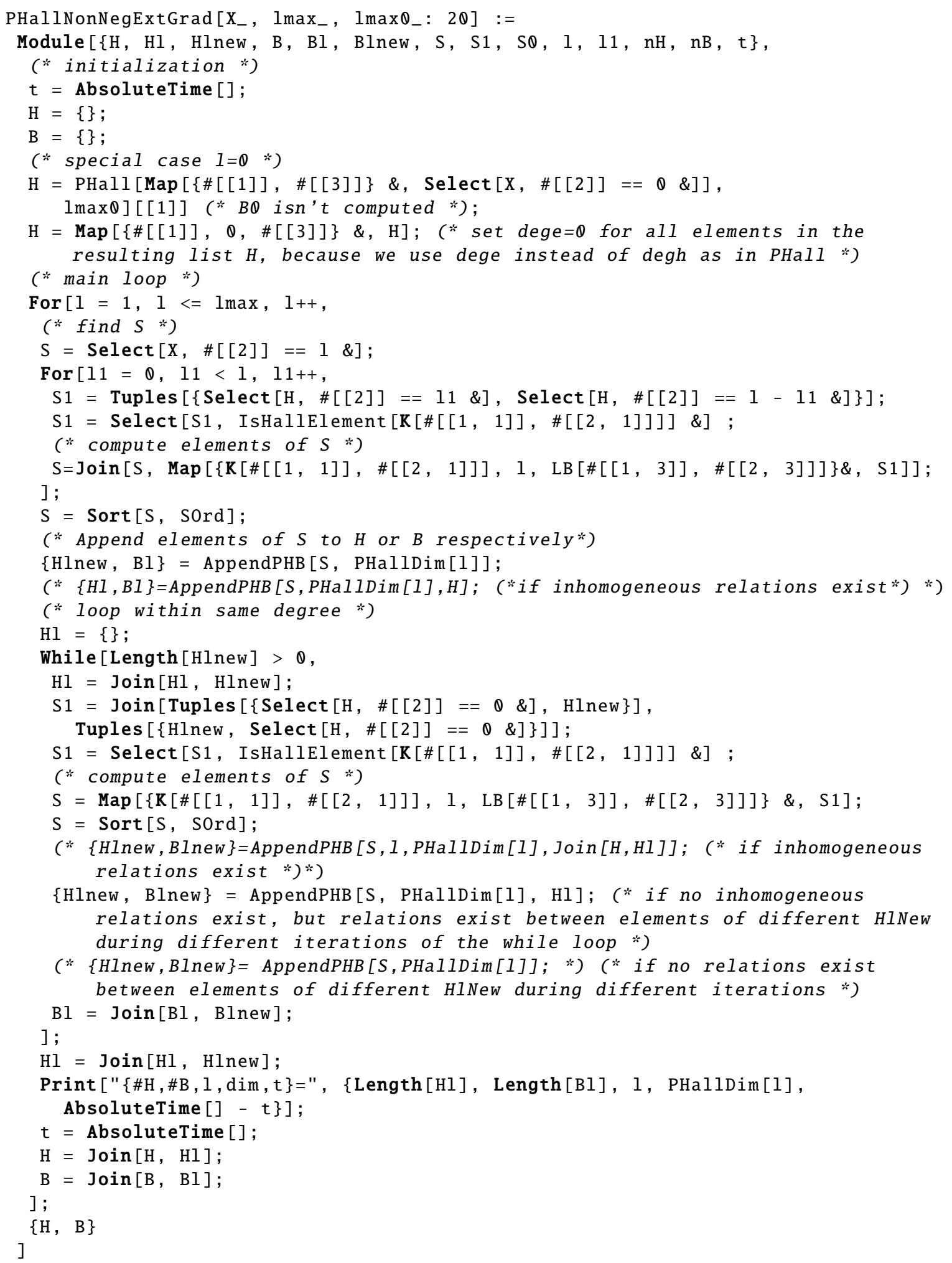




\section{Appendix B}

\section{Mathematica Code for the Pohlmeyer-Rehren Lie algebra}

This section documents a large part of the Mathematica code developed for calculations in the Pohlmeyer-Rehren Lie algebra for this thesis. For most modules, examples are given that can also serve as unit tests for reimplementation in other languages. Some of the code used here had previously been developed by the author for his Diplomarbeit [Han09].

\section{B.1 Shuffle product}

The code used here to calculate the shuffle product is taken from user ciaO at the Mathematica forum at stackexchange.com 1 . This algorithm was chosen because it performs significantly better than the one used in [Han09] which was based on the recursive formula for the shuffle product 1.1 .4

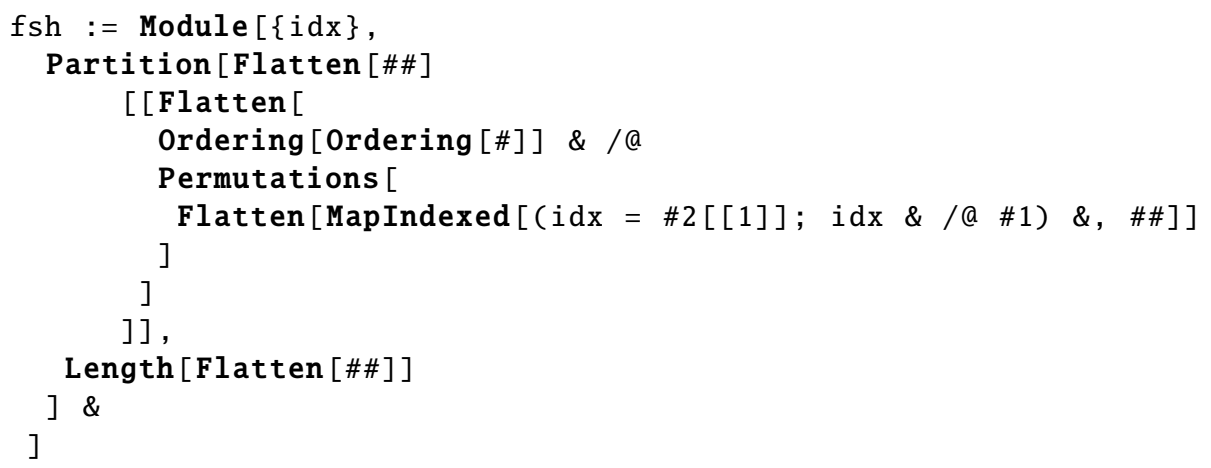

Since this source does not give an explanation how this implementation works, one is given here. Let us consider the calculation of the shuffle product of the words $a b$ and $x y z$, the list of which is represented as

lists $=\{\{a, b\},\{x, y, z\}\}$

First, generate a list of placeholders, denoted by the index of a word a letter is taken from (in the list of words to be shuffled), for the letters of the words that are to be shuffled, in our example

\footnotetext{
${ }^{1}$ URL: https://mathematica.stackexchange.com/questions/41614/shuffle-product-of-two-lists
} 
Flatten [MapIndexed [(idx $=\# 2[[1]] ;$ idx \&/@\#1) \&, \#\#] \&[lists]

yields $\{1,1,2,2,2\}$. Then generate all permutations of this list of placeholders. This is the most computationally expensive part of the computation of the shuffle product, but it is fast because it is done by the built-in function Permutations written in a lower level programming language. In our example,

Permutations [Flatten [MapIndexed $[(i d x=\# 2[[1]] ;$ idx \&/@ \#1)\&, \#\#]] \&[lists]

yields

$\{\{1,1,2,2,2\},\{1,2,1,2,2\},\{1,2,2,1,2\},\{1,2,2,2,1\},\{2,1,1,2,2\}$,

$\{2,1,2,1,2\},\{2,1,2,2,1\},\{2,2,1,1,2\},\{2,2,1,2,1\},\{2,2,2,1,1\}\}$

The rest of the code replaces the placeholders by the letters of the words to be shuffled, in the correct order relative to the respective words. In our example, fsh [lists] yields

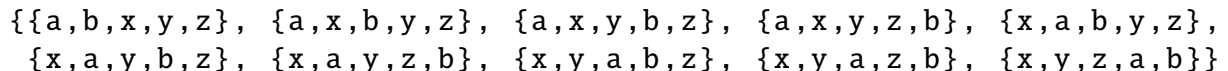

Note that the result is a list (of summands) because Mathematica treats a sum of lists of the same length as a sum of vectors.

A generalization of $f$ sh to concatenations of shuffle products of (an arbitrary number of) words is the module rsh:

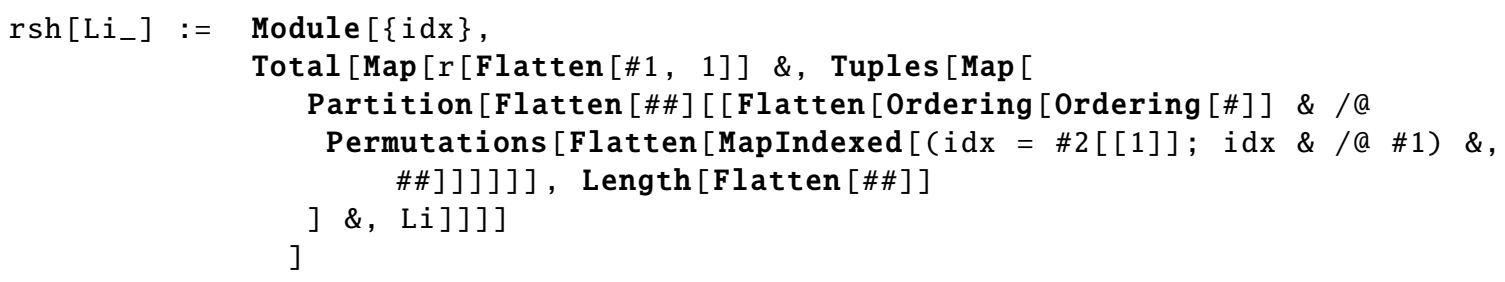

This is equivalent to Total $[\operatorname{Map}[r[F \operatorname{latten}[\# 1,1]] \&$, Tuples $[\operatorname{Map}[f \operatorname{sh}, \mathrm{Li}]]]]$, but slightly faster as it avoids function calls. Since in our application we actually want to obtain Eulerian idempotents of such terms, i.e. terms of the form

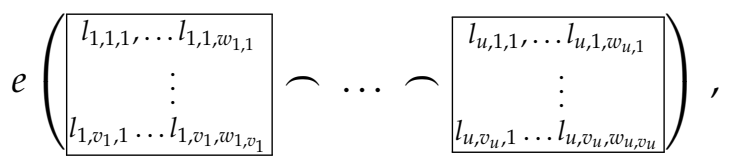

we use the list header $r$ for the Eulerian idempoten $t^{2}$, which also allows us to use the built-in function Total to formally sum up the different summands of the shuffle product.

Example B.1.1. $\operatorname{rsh}[\{p, q\},\{\{\{a, b\},\{c\}\},\{\{x\},\{y\}\}\}]$ yields

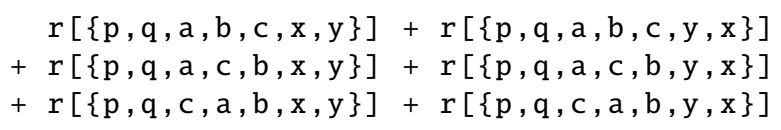

Finally, a version with Euler-Lyndon elements as its output is introduced:

\footnotetext{
${ }^{2}$ The letter $r$ was chosen corresponding for the old notation for the truncated tensors, cf. section 3.1 because e was already in use by Mathematica for the built-in Eulerian constant.
} 


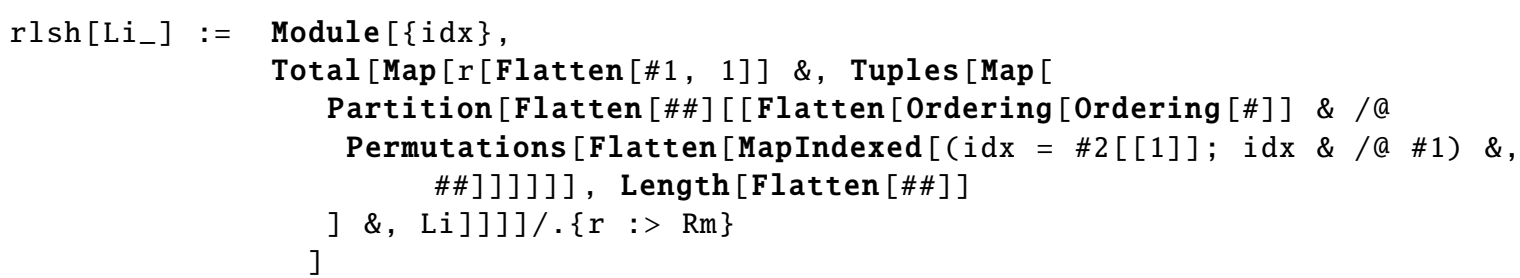

\section{B.2 Rewriting Euler elements in terms of Euler-Lyndon elements}

\section{B.2.1 Rm}

Rm implements Pohlmeyer's and Rehren's algorithm (see remark 1.3.3). Its argument is a word $x$, written as a list of letters Li, and it returns $e(x)$ written as a linear combination of Euler-Lyndon elements. Results are stored in RAM since (for long words) retrieval is several orders of magnitude faster than calculation.

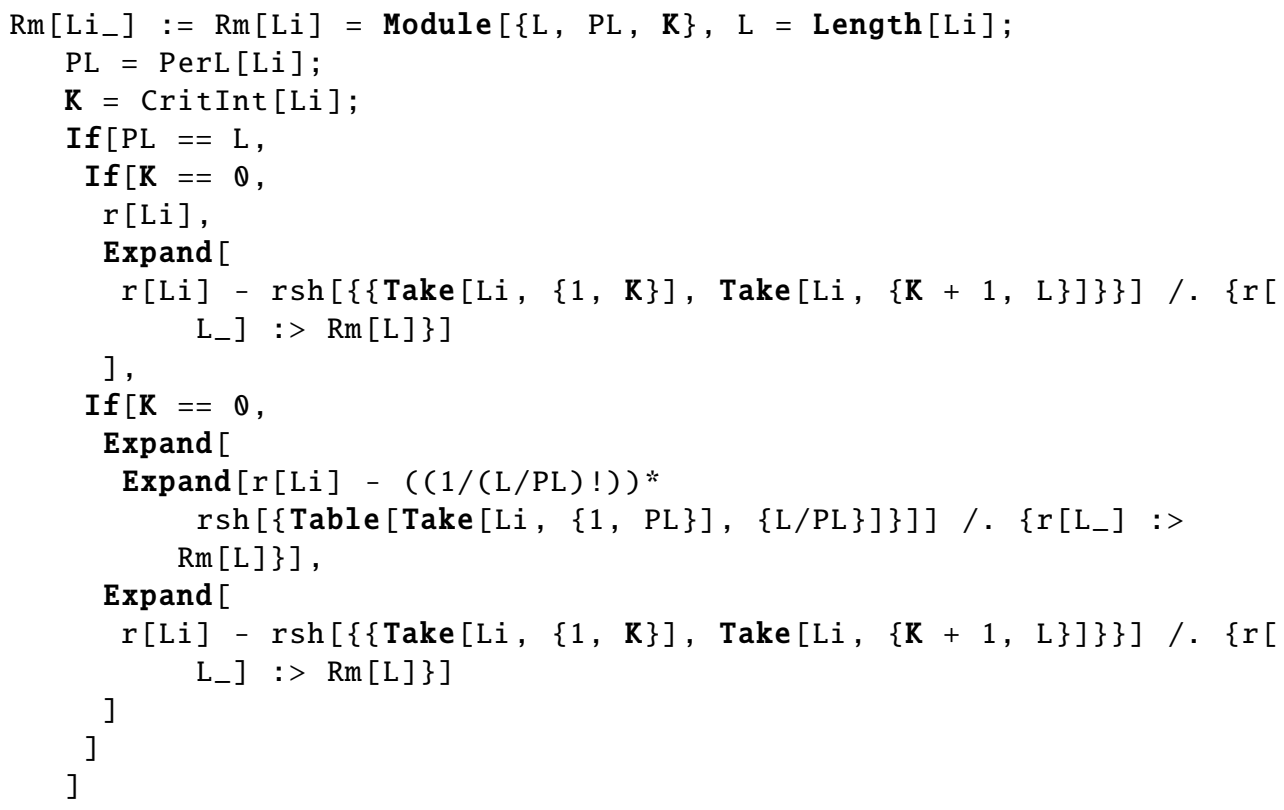

EulLynMem allows to apply this as a rule.

EulLynMem $=\left\{r\left[\mathrm{Li}_{-}\right]:>\mathrm{Rm}[\mathrm{Li}]\right\}$

Example B.2.1. $r[\{1,0,2\}] /$. EulLynMem is resolved to $\operatorname{Rm}[\{1,0,2\}]$, which yields $-r[\{\theta, 1,2\}]-\operatorname{r}[\{\theta, 2,1\}]$ 


\section{B.2.2 PerL}

PerL takes as its input a a word, represented as a list $\mathrm{Li}$, and returns its period length.

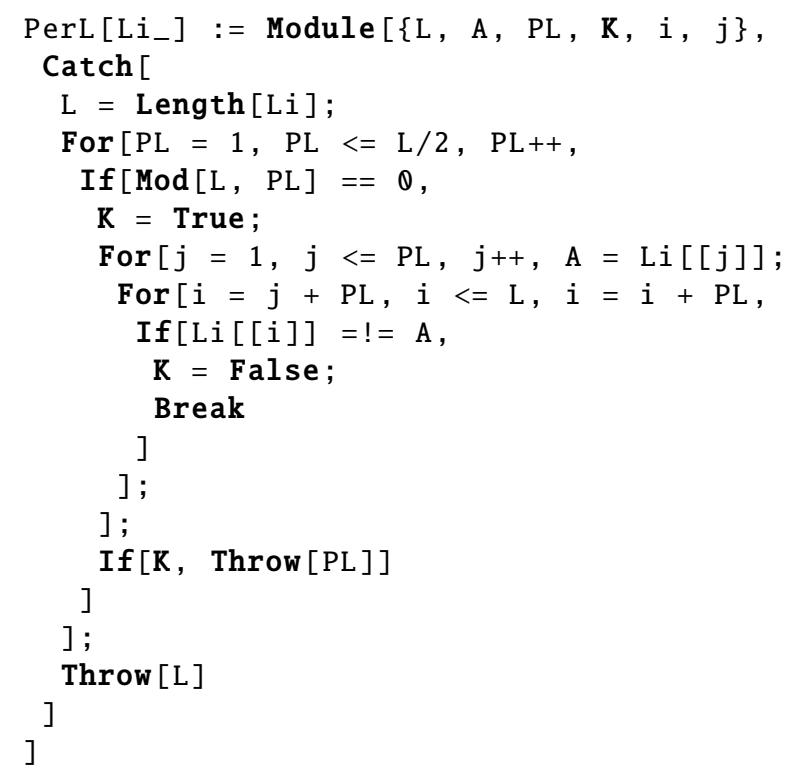

Example B.2.2. PerL $[\{0,1,0,1,0,1\}]$ yields 2 .

\section{B.2.3 CritInt}

CritInt takes as its input a a word, represented as a list Li, and returns its critical integer (indicating how many positions Li has to be shifted (to the left) in order to be minimal among all cyclic rotations) with respect to the order of letters Lett0rd.

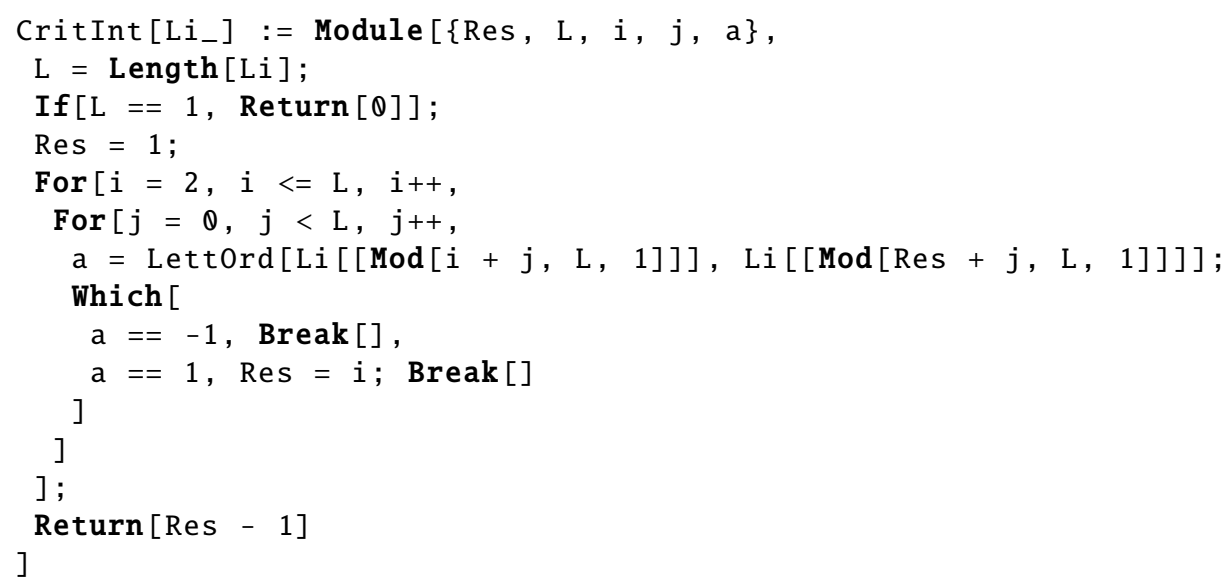

Example B.2.3. CritInt $[\{2,0,1\}]$ yields 1 . 


\section{B.2.4 LettOrd}

Lett0rd implements an order on an alphabet by taking the order explicitly given from the list Lett0rdList. Letters not on this list are considered greater than letters on it, and two letters not on the list are compared using the built-in function Order. The output is in the standard Mathematica format of Order (see A.1.3).

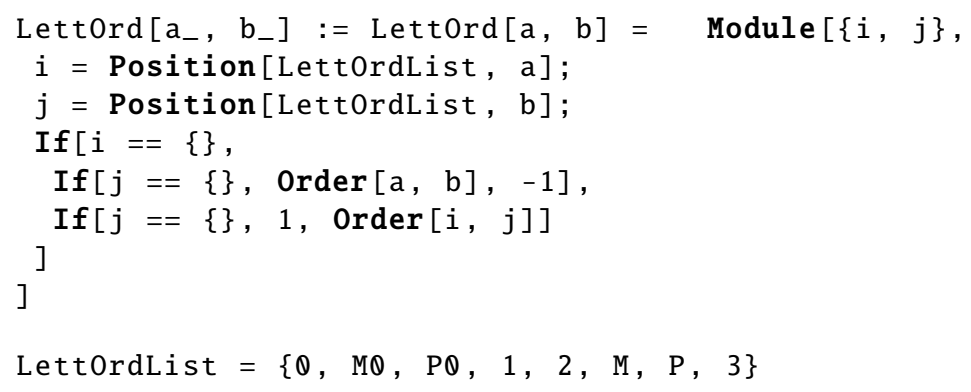

Example B.2.4. LettOrd[MO, P] yields 1.

\section{B.2.5 LyndonQ}

LyndonQ takes as its input a word, represented as a list $\mathrm{Li}$, and returns True if $\mathrm{Li}$ is a Lyndon word with respect to the order of letters LettOrd, False otherwise. It uses CritInt to check if $\mathrm{Li}$ is minimal among its cyclic rotations and PerL to check if it is nonperiodic (i.e. uniquely minimal if it is minimal).

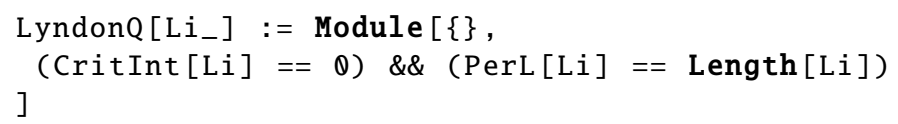

\section{B.2.6 Lyndon}

Lyndon accepts as input a list of letters Elements and integers nmin and (optionally) nmax. The output is a list of all Lyndon words, written as lists, of length $n$ such that $n$ min $\leq n \leq n$ max. If no nmax is supplied, nmax:= nmin is used.

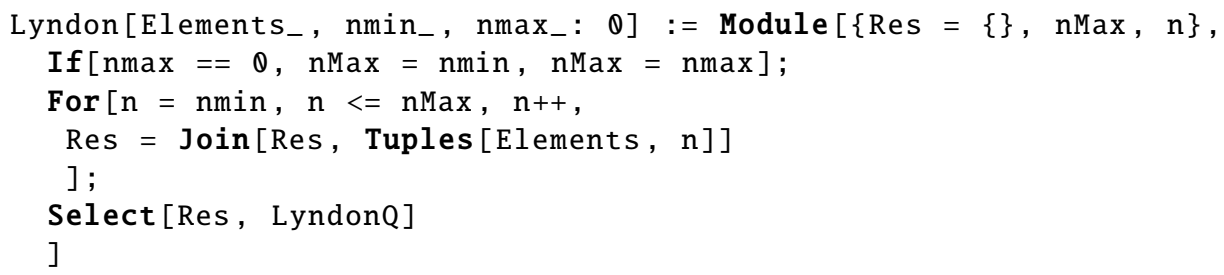

Example B.2.5. Lyndon $[\{\boldsymbol{\theta}, 1,2\}$, 3] with standard order of natural numbers yields $\{\{\boldsymbol{\theta}, \boldsymbol{\theta}, 1\},\{\boldsymbol{\theta}, \boldsymbol{\theta}, 2\},\{\boldsymbol{\theta}, 1,1\},\{\boldsymbol{\theta}, 1,2\},\{\boldsymbol{\theta}, 2,1\},\{\boldsymbol{\theta}, 2,2\},\{1,1,2\},\{1,2,2\}\}$

\section{B.2.7 rLyndon}

rLyndon applies the formal function $r$ to the result of Lyndon to output all Euler-Lyndon elements of desired length. Imput conventions are the same as for Lyndon above.

rLyndon [par_-- $]:=\operatorname{Map}[r, \operatorname{Lyndon}[$ par] $]$ 


\section{B.3 Dimension formulas}

\section{B.3.1 NumLyndon}

NumLyndon is a straightforward implementation of the formula from theorem 1.3.61. It accepts integers $\mathrm{n}$ and $\mathrm{d}$ and returns the number of Lyndon words of length $n$ over an alphabet of $d$ letters.

NumLyndon $\left[n_{-}, d_{-}\right]:=1 / n * D i v i \operatorname{sor} \operatorname{Sum}\left[n, \operatorname{MoebiusMu}[\#]^{*} d^{\wedge}(n / \#) \&\right]$

\section{B.3.2 NumLyndonL}

NumLyndonL is a corresponding implementation of the formula from theorem 1.3.62. It accepts a list of integers $L$ and returns the number of Lyndon words featuring exactly L [ [i] ] occurrences of the $i$-th letter.

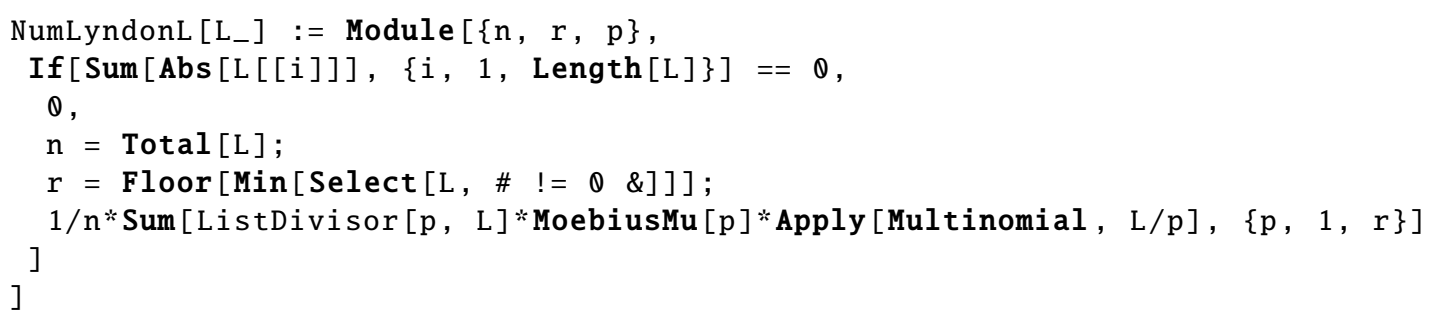

It uses the module ListDivisor which accepts an integer $d$ and a list of integers $L$ and returns 1 if $d$ is a divior of each of the entries of $L$, and returns $\theta$ otherwise.

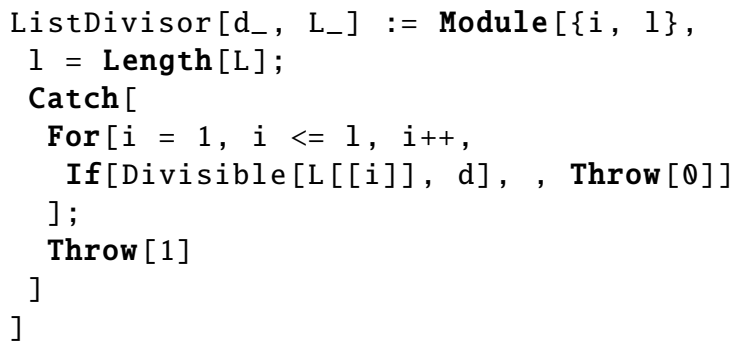

Example B.3.1. NumLyndonL $[\{1,1,2\}]$ returns 3 because (irrespective of the order) there are 3 different Lyndon words in which one letter appears twice and two other letters appear once, for instance

$$
\text { (0012), (0021), (0102) . }
$$

\section{B.3.3 Dimensions of weight spaces and numbers of multiplets for $d=3$}

WSDim3 implements formula (2.94) for the dimension of weight spaces for $d=3$.

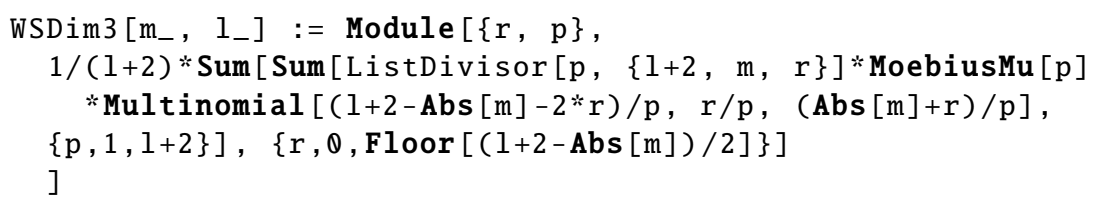


NumMults3 gives the number $v_{l}(s)$ of multiplets of highest weight $\mathrm{s}$ in stratum $l$ for $d=3$ according to corollary 2.3.134.

NumMults $3\left[s_{-}, 1_{-}\right]:=$

WSDim3m[Abs $[s], 1]-\operatorname{WSDim} 3 \mathrm{~m}[\operatorname{Abs}[\mathrm{s}]+2,1]$

\section{B.3.4 Dimensions of weight spaces and numbers of multiplets for $d=4$}

WSDim4m implements formula (2.92) for the dimension of weight spaces denoted by magnetic quantum numbers $m_{0}, m_{1}$ in stratum 1 for $d=4$.

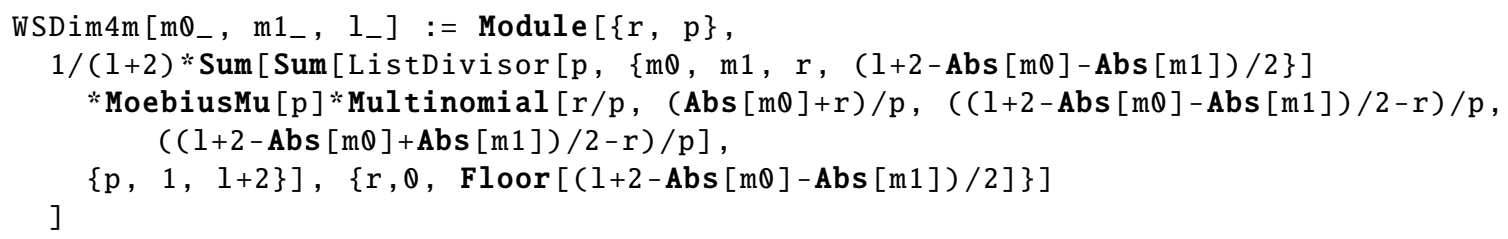

WSDim4my implements the same formula for weights denoted by weight tuples my $\mathbb{Q}$, my 1 .

WSDim4my $\left[m y \theta_{-}, \operatorname{my} 1_{-}, 1_{-}\right]:=\operatorname{WSDim} 4 \mathrm{~m}[(\operatorname{my} \theta-m y 1) / 2,(\operatorname{my} \theta+m y 1) / 2,1]$

NumMults 4 implements formula (2.108) for the number of multiplets of highest weight $\left(\sigma_{0}, \sigma_{1}\right)$ in stratum $l$ for $d=4$.

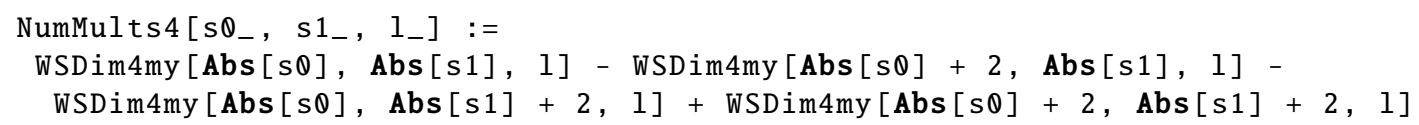

\section{B.4 Pohlmeyer-Rehren Lie bracket}

\section{B.4.1 g}

g implements the Minkowski metric (cf. definition 1.4.3) with $\alpha=1$. A different (or symbolic) $\alpha$ can be implemented easily by writing it as a factor before the Which statement. Note that since + and - are operators in Mathematica that cannot be overloaded, we use $M[i]$ and $\mathrm{P}[\mathrm{i}]$ for $-{ }_{i}$ and $t_{i}$ respectively. To save typing for $d=3,4$, the abbreviations $\mathrm{M}=\mathrm{M} 1=\mathrm{M}[1]$, $\mathrm{P}=\mathrm{P} 1=\mathrm{P}[1], \mathrm{M} \mathrm{Q}=\mathrm{M}[\mathrm{\theta}], \mathrm{P} \mathrm{Q}=\mathrm{P}[\mathrm{\theta}]$ can be used.

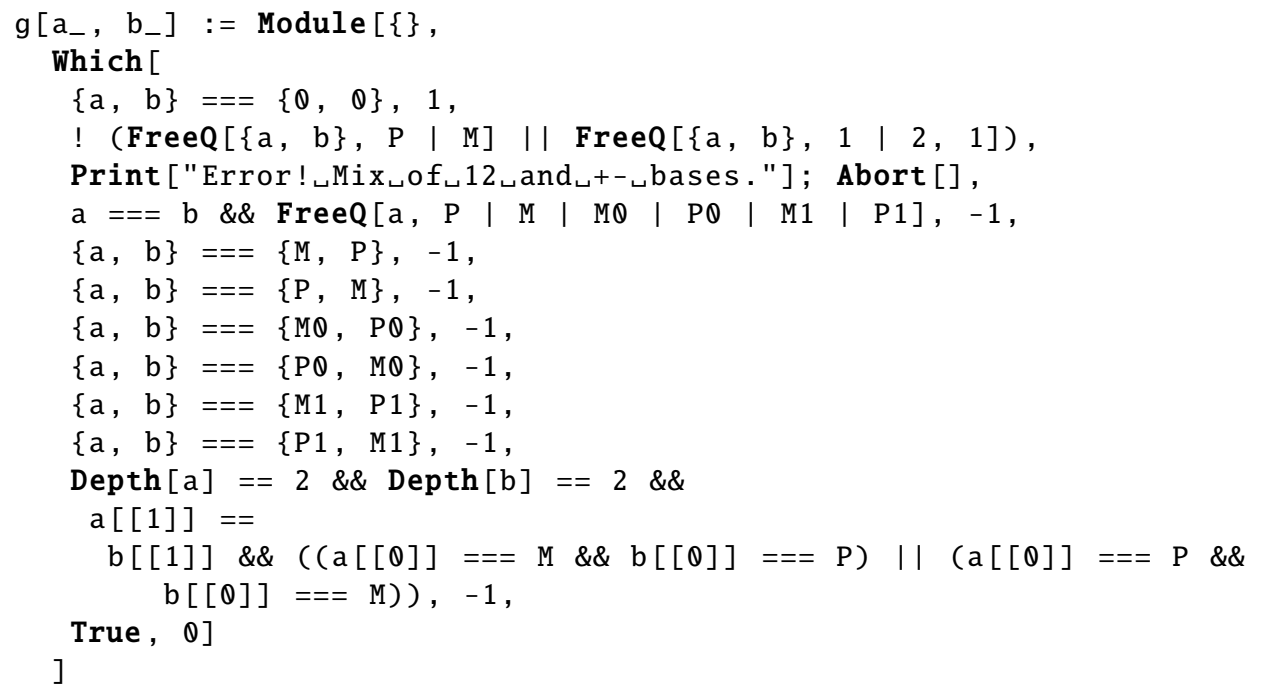




\section{B.4.2 Lm}

Lm implements the Pohlmeyer-Rehren Lie bracket (1.54) of Euler elements. Note that the result is written in terms of Euler-Lyndon elements using Pohlmeyer's algorithm. After this computation, results are stored in memory so that the repeated computations of the same Lie bracket can be avoided.

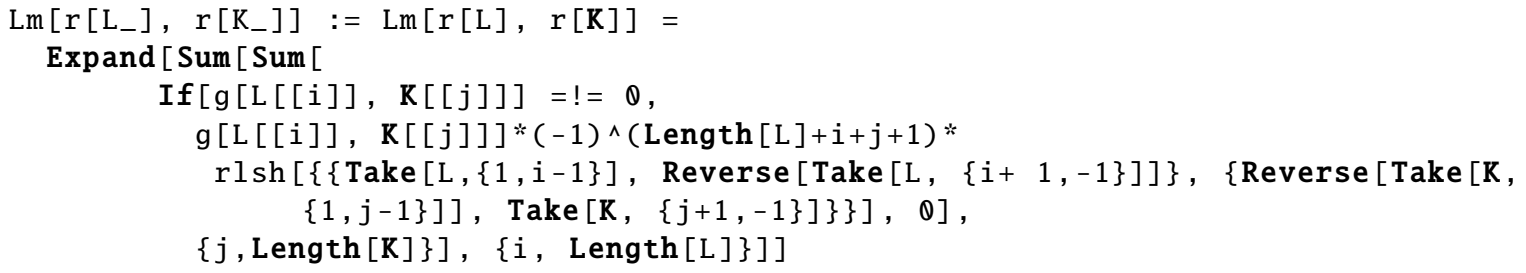

\section{B.4.3 LieRules}

LieRules is a set of rules that encompasses linearity of the Lie bracket as well as the Lie bracket of individual Euler elements. It can be used to calculate (multiple) arbitrary PohlmeyerRehren Lie brackets.

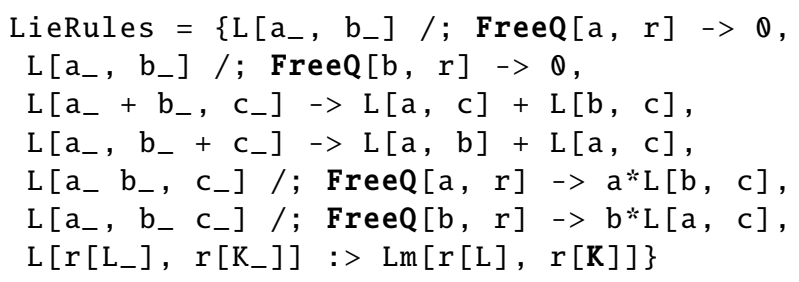

\section{B.4.4 LB}

LB uses the set of rules LieRules to calculate the Lie bracket of two elements of $\mathfrak{g}$.

LB $\left[a_{-}, b_{-}\right]:=L[a, b] / /$. LieRules

Example B.4.1. $\operatorname{LB}[r[\{\boldsymbol{\theta}, \mathrm{P}\}], \mathrm{r}[\{\mathrm{M}, \mathrm{P}\}]]$ yields $2 \mathrm{r}[\{\boldsymbol{\theta}, \mathrm{P}\}]$.

\section{B.5 Linear operators}

Linear operators that are of particular interest to the application to the Pohlmeyer-Rehren Lie algebra for $d=3$ are the angular momentum operator $J=\operatorname{ad}_{h}$, the ladder operators $J^{+}=\operatorname{ad}_{x}, J^{-}=\operatorname{ad}_{y}$ and the Casimir operator $J^{2}=J^{+} J^{-}+J^{-} J^{+}+J J$. The angular momentum and Casimir operators commute and for $x \in{ }^{s} \mathrm{~g}^{m}$ the eigenvalue equations

$$
\begin{aligned}
J x & =m \cdot x, \\
J^{2} x & =s(s+1) \cdot x
\end{aligned}
$$

hold. 


\section{B.5.1 Angular momentum operator $J$}

J $=\operatorname{Expand}[\mathrm{LB}[\mathrm{r}[\{\mathrm{M}, \mathrm{P}\}], \#]] \&$

\section{B.5.2 Ladder operators $J^{ \pm}$}

$J P=\operatorname{Expand}[\operatorname{LB}[r[\{\theta, P\}], \#]] \&$

$J M=\operatorname{Expand}[\operatorname{LB}[r[\{\theta, M\}], \#]] \&$

\section{B.5.3 Casimir operator $J^{2}$}

$J 2=J P[J M[\#]]+J M[J P[\#]]+J[][\#]] / /$. EulLynMem \&

\section{B.6 Linear algebra tools}

The Mathematica modules presented in this section are mainly wrappers around built-in functions that are geared towards improved usability for the Pohlmeyer-Rehren Lie algebra.

\section{B.6.1 BaseVars}

BaseVars takes as its input a list L of elements (that aren't necessarily linearly independent) of the Pohlmeyer-Rehren Lie algebra and returns a basis of the vector space spanned by $\mathrm{L}$.

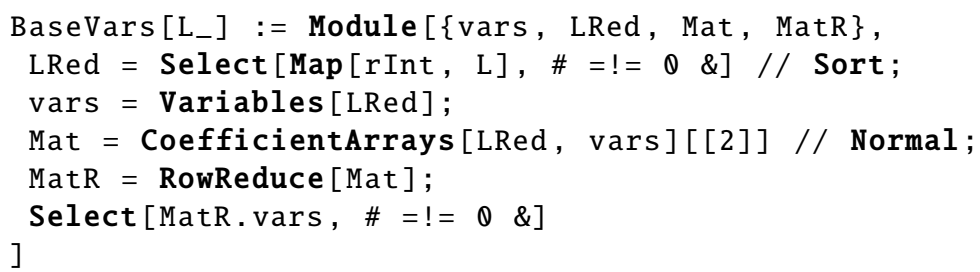

Example B.6.1. BaseVars $[\{r[\{0, M, P\}]+r[\{0, P, M\}], r[\{0, M, P\}]-r[\{0, P, M\}]$, $7 * \mathrm{r}[\{\theta, M, P\}]+2 * \mathrm{r}[\{\theta, \mathrm{P}, \mathrm{M}\}]\}]$

yields

$\{r[\{\theta, M, P\}], \quad r[\{\theta, P, M\}]\}$

BaseVars uses the module rInt, which finds a multiple of a given element of $\mathfrak{g}$ with integer coefficients with respect to the Euler-Lyndon basis.

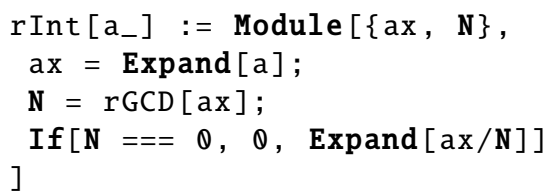


rInt in turn uses the module rGCD to find the greatest common denominator of the coefficients of a linear combination of Euler-Lyndon elements.

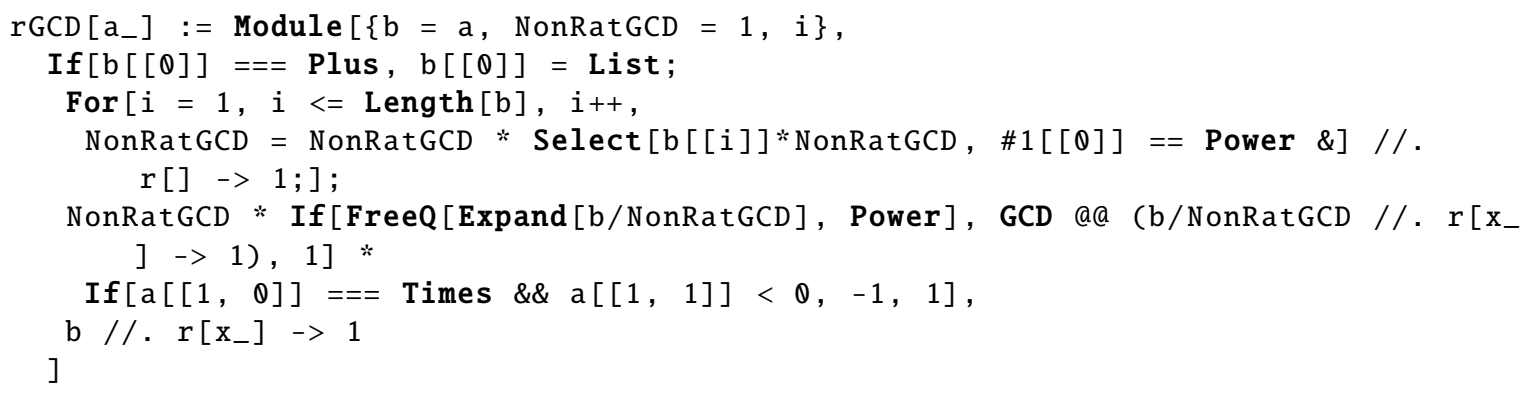

\section{B.6.2 LinCoeffs}

LinCoeffs accepts as its input a basis, represented as a list $B$, and a vector $\mathrm{v}$ from the vector space spanned by the basis and returns the list of linear coefficients of $\mathrm{v}$ relative to $\mathrm{B}$.

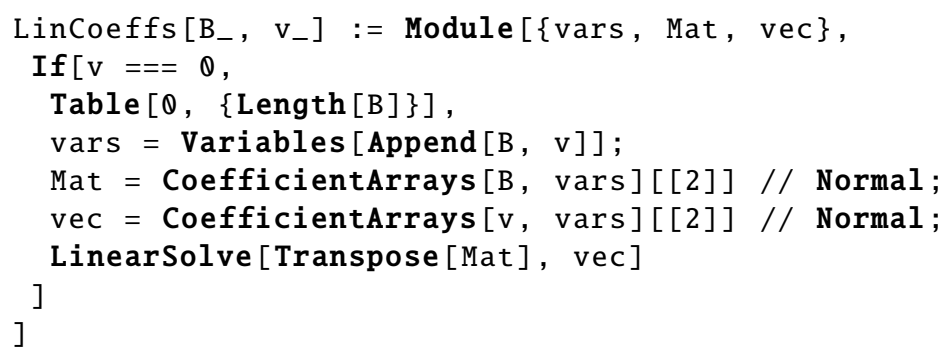

Example B.6.2. LinCoeffs $[\{r[\{\theta, M\}]+r[\{\theta, P\}], \quad r[\{\theta, P\}]\}, \quad r[\{\theta, M\}]]$ yields $\{1,-1\}$

\section{B.6.3 EigSps}

EigSps accepts a list of basis vectors LIn spanning a vector space and an endomorphism $f$ of that space and returns a list of all eigenvalues and corresponding eigenspaces (given as a list of basis vectors).

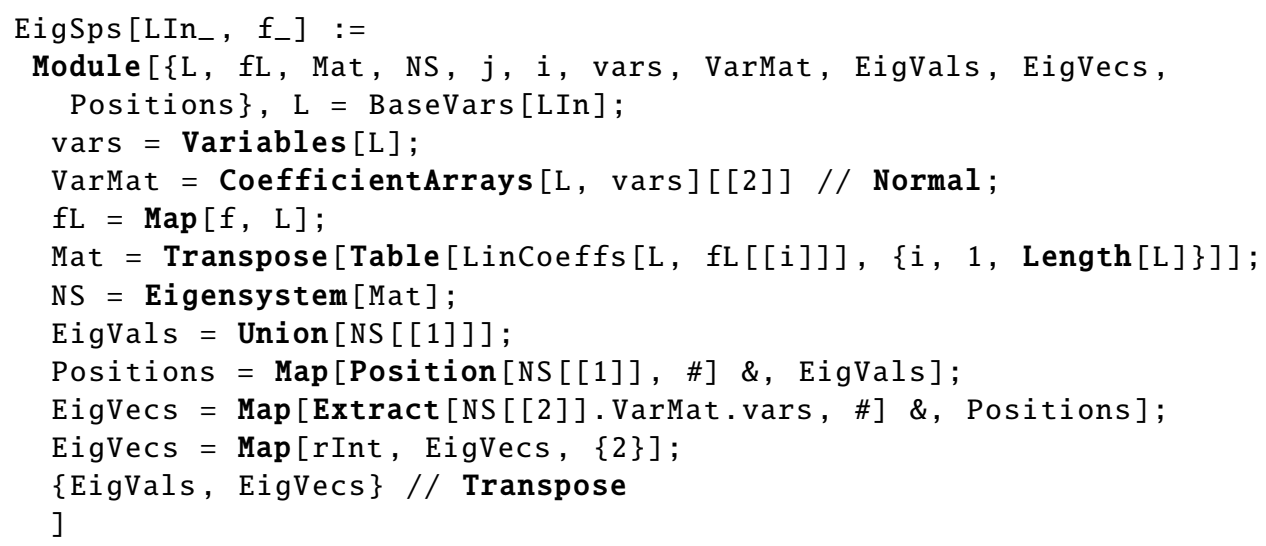




\section{B.6.4 SimEigSps}

SimEigSps is a generalization of EigSps in that it accepts a list Homs of endomorphisms in addition to a vector space, given as a list Vecs of basis vectors, and calculates simultaneous eigenvectors (and corresponding eigenvalues) with respect to all given endomorphisms.

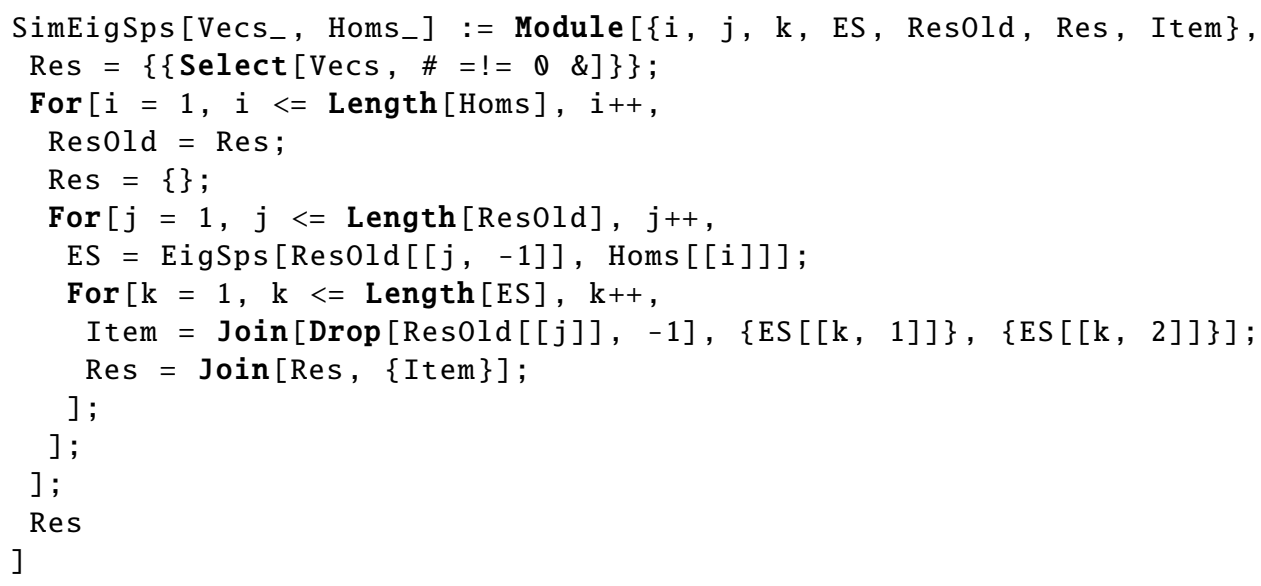

Example B.6.3. We calculate the simultaneous eigenspaces of the of the Casimir operator $J^{2}$ and the angular momentum operator $J$ on a stratum $\mathfrak{g}_{l}$ for $d=3$. Note that the eigenspaces of the Casimir operator are the direct sums of all irreducible $\mathfrak{g}_{0}$-modules of a given highest weight vector and the eigenspaces of $J$ are the weight spaces (cf. remark 2.3.9. Thus, we obtain the decomposition

$$
\mathfrak{g}_{l}=\bigoplus_{s=0}^{l+1} \bigoplus_{m=-s}^{s}{ }^{s} \mathfrak{g}_{l}^{m} .
$$

Note that this is only a decomposition into irreducible $\mathfrak{g}_{0}$-modules if all multiplicities are 1, i.e. if $l \leq 1$. To obtain that, one would have to make a choice for a basis of a given weight space (cf. remark 2.3.17) consistent with the action of the ladder operators. To calculate the above decomposition for $l=2$ in Mathematica we can use the command

SimEigSps [rLyndon $[\{\boldsymbol{\theta}, \mathrm{M}, \mathrm{P}\}, 4],\{] 2, J\}] / /$ Grid, which yields

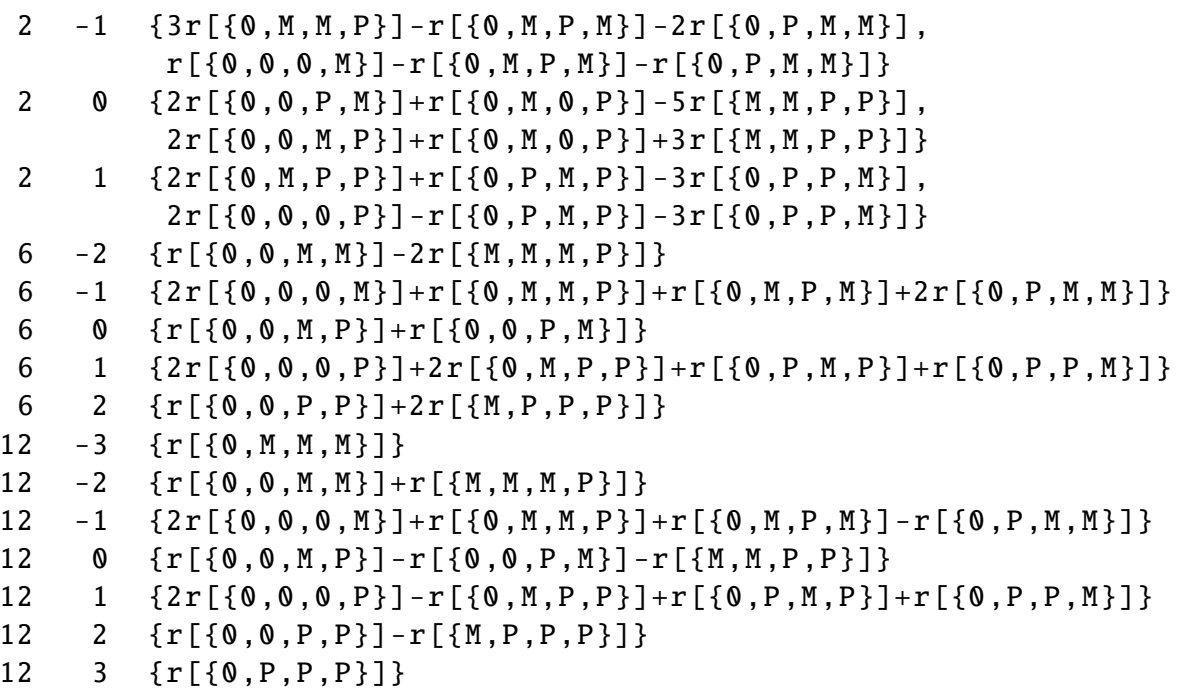




\section{B.6.5 dimension}

dimension accepts as its input a vector space $V$ given as a list $\mathrm{L}$ of the vectors that span $V$ and returns $\operatorname{dim} V$.

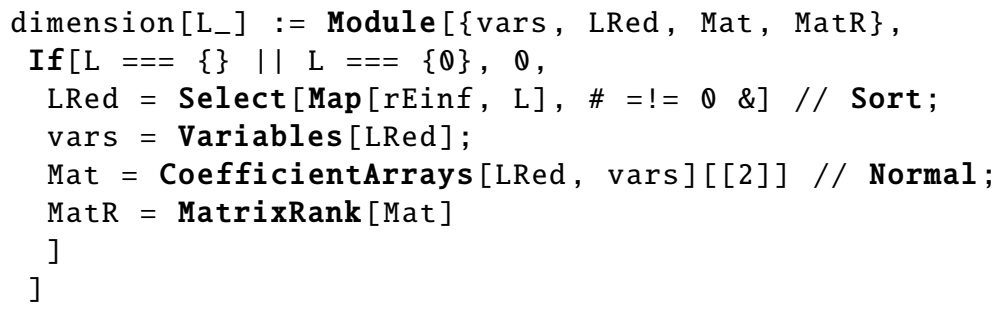

\section{B.6.6 Kernel}

Kernel accepts as its input a basis of a vector space $V$ given as a list $\mathrm{L}$ and a linear function $f: V \rightarrow W$ (for some vector space $W$ and returns the Kernel ker $f$ as a list of basis vectors.

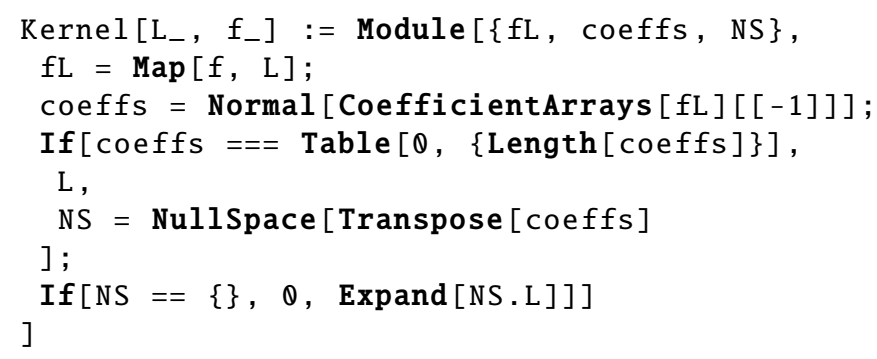

\section{B.6.7 Kernels}

Kernels accepts as its input a basis of a vector space $V$ given as a list L and a list Lf of linear functions $V \rightarrow W$ (for some vector space $W$ and returns the intersection of the kernels $\bigcap_{f \in \mathrm{Lf}} \operatorname{ker} f$ as a list of basis vectors.

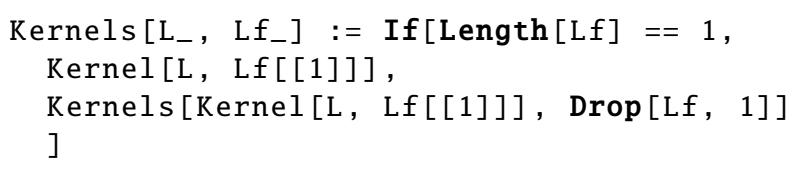

Example B.6.4. We calculate the highest weight vectors of $\mathfrak{g}_{1}$ for $d=4$, as in remark 2.3.15. A linear basis of $g_{1}$ in the spin basis is given by $\left.\operatorname{rLyndon}[\{\mathrm{MQ}, \mathrm{PQ}, \mathrm{M}, \mathrm{P}\}], 3\right]$ and elements associated to positive roots are $x_{0}=e\left(+_{0}+\right), x_{1}=e\left(-_{0}+\right)$, so (using anonymous functions for adjunctions of the $x_{i}$ ),

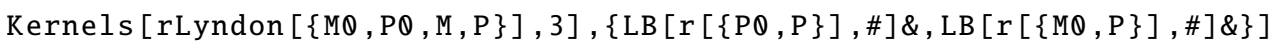

calculates the highest weight vectors, specifically

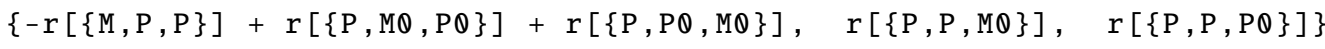




\section{B.6.8 CenterTest}

CenterTest was used to test the conjecture 2.1.3 that the center of the Pohlmeyer-Rehren Lie algebra is identical to $\mathfrak{g}_{-1}$. It takes as its input a list of elements of the Pohlmeyer-Rehren Lie algebra $\mathrm{L}$ and iteratively calculates the intersection of the kernels of the adjunctions of the elements of L by iteratively calculating the kernel of the restriction of an adjunction to the intersection of the kernels previously considered.

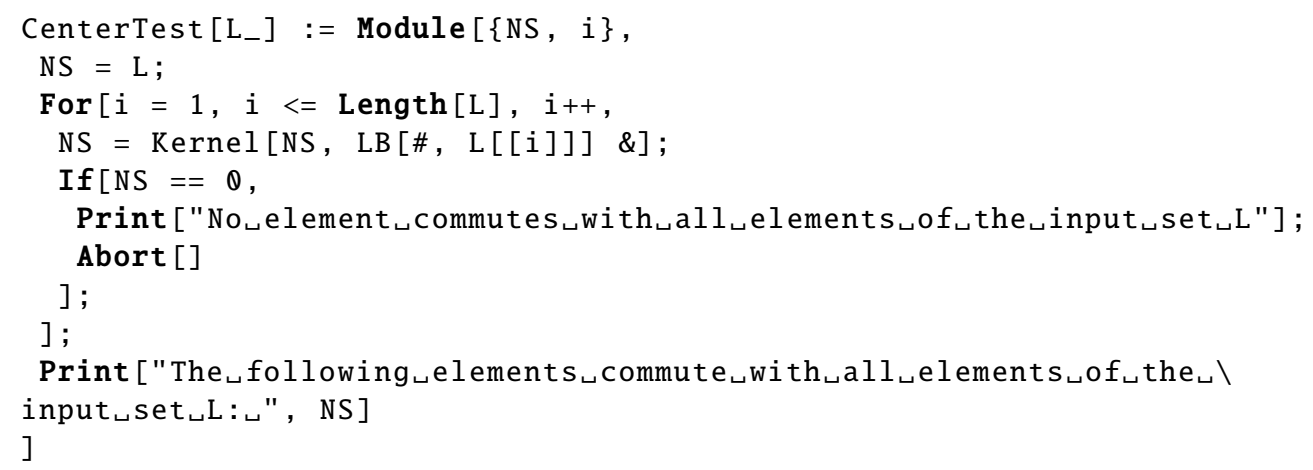




\section{Appendix C}

\section{Calculations Regarding the Generation of Leading Terms of Exceptional Elements in $\hat{\mathfrak{h}}$}

This appendix serves to outline the partial results obtained in the pursuit of a proof of conjecture 3.4.2. While that attempt was not successful, the method deserves to be described.

Conjecture 3.4 .2 states that the leading term of the exceptional element $L_{(1)}$ with $l=2 n+3$ can be found in the quantum correction of

$$
\left\{\ldots\{\left\{L_{-2}, L_{-1}\right\}, \underbrace{\left.\left.L_{0}\right\}, \ldots, L_{0}\right\}}_{n} \cdot\left\{\ldots\{\left\{L_{2}, L_{1}\right\}, \underbrace{\left.\left.L_{0}\right\}, \ldots, L_{0}\right\}}_{n} \in \mathfrak{h}_{2 n+5} \cdot\right.\right.
$$

According to the quantization procedure laid out in chapter 3 , a quantized lifting of the above invariant is given by the anticommutator

$$
\begin{aligned}
& \frac{1}{2}\left(\left[\ldots[\left[\hat{L}_{-2}, \hat{L}_{-1}\right], \underbrace{\left.\left.\hat{L}_{0}\right], \ldots, \hat{L}_{0}\right]}_{n} \cdot\left[\ldots[\left[\hat{L}_{2}, \hat{L}_{1}\right], \underbrace{\left.\left.\hat{L}_{0}\right], \ldots, \hat{L}_{0}\right]}_{n}\right.\right.\right. \\
& +\left[\ldots[\left[\hat{L}_{2}, \hat{L}_{1}\right], \underbrace{\left.\left.\hat{L}_{0}\right], \ldots, \hat{L}_{0}\right]}_{n} \cdot\left[\ldots[\left[\hat{L}_{-2}, \hat{L}_{-1}\right], \underbrace{\left.\left.\hat{L}_{0}\right], \ldots, \hat{L}_{0}\right]}_{n}) \in \hat{\mathfrak{h}}_{2 n+5} .\right.\right.
\end{aligned}
$$

In the rest frame, the $\hat{L}_{i}$ are sums of terms of the form me(abc) and $e(a b) e(c d)$ (with constant coefficients) and a quantum correction, where $a, b, c, d \in X$. By linearity, each of the products above can be expanded to a linear combination of summands that inherit only one of those terms from each $L_{i}$. We can now use the special form of lead $L_{(1)}$ to identify a large number of summands that cannot contribute to it. Note that the quantum corrections of the individual $\hat{L}$ are of sufficiently low degree that any summand of the expansion of the above equation containing at least one of them cannot contribute to lead $L_{(1)}$. For reasons of degree, only those summands where exactly one term in a multiple Lie bracket is of the form $e(a b) e(c d)$ and all the other terms are of the form $m \cdot e(a b c)$ can contribute to the leading term of the exceptional element. Combining the above considerations and ignoring the common factor $1 / 2$ from the anticommutator, all summands that can possibly contribute to lead $L_{(\mathbb{D}}$ are of 
the form

$$
\begin{gathered}
\overbrace{\left.\left[\ldots\left[\left[x_{-2}, x_{-1}\right]\right], x_{0,-1}\right], \ldots, x_{0,-n}\right]}^{=: F_{-1}} \cdot \overbrace{\left[\ldots\left[\left[x_{2}, x_{1}\right], x_{0,1}\right], \ldots, x_{0, n}\right]}^{=: F_{+2}} \\
+\underbrace{\left.\left[\ldots\left[\left[x_{2}, x_{1}\right]\right], x_{0,1}\right], \ldots, x_{0, n}\right]}_{=: F_{+1}} \cdot \underbrace{\left[\ldots\left[\left[x_{-2}, x_{-1}\right], x_{0,-1}\right], \ldots, x_{0,-n}\right]}_{=: F_{-2}},
\end{gathered}
$$

where exactly one of the $x$ must be of the form $e(a b) e(c d)$ while all the others are of the form $m e(a b c)$. To evaluate the sum of these terms, we need to look at each individual summand, designated by which of the $x$ is of the form $e(a b) e(c d)$. The $m$ are suppressed in the calculations because they are central and can therefore be treated as constant factors, and we call the $x$ of the form $e(a b) e(c d)$ the quadratic $x$.

We first prove a general lemma that is simply a formalization of the iterative application of Leibniz's rule to multiple Poisson brackets with a single product in them, just as in the above situation.

Lemma C.0.1. Let $(H, \cdot\{\}$,$) be a Poisson algebra. Then the following equation for multiple brackets$ holds: if $z_{i}, y_{j} \in H$ for all $i \in\{1,2\}, j \in\{1, \ldots, n\}$, then

$$
\left\{\ldots\left\{\left\{z_{1} \cdot z_{2}, y_{1}\right\}, y_{2}\right\}, \ldots, y_{n}\right\}=\sum_{s \in\{0,1\}^{n+1}} l(s) \cdot r(s)
$$

with

$$
\begin{aligned}
l(()) & =z_{1}, \\
r(()) & =z_{2}, \\
l(s, 0) & =l(s), \\
r(s, 0) & =\left\{r(s), y_{k}\right\}, \\
l(s, 1) & =\left\{l(s), y_{k}\right\}, \\
r(s, 1) & =r(s)
\end{aligned}
$$

for $s \in\{0,1\}^{k-1}$.

Proof. Induction over $n$. For $n=0$, this is Leibniz's rule. Now suppose the equation holds up to $n$. Then,

$$
\begin{aligned}
\left\{\ldots\left\{\left\{z_{1} \cdot z_{2}, y_{1}\right\}, y_{2}\right\}, \ldots, y_{n+1}\right\}= & \left\{\ldots\{z_{1} \cdot \underbrace{\left.z_{2}, y_{1}\right\}}_{=: z_{2}^{\prime}}, y_{2}\}, \ldots, y_{n+1}\right\}+\left\{\ldots\{\underbrace{\left\{z_{1}, y_{1}\right\}}_{=: z_{1}^{\prime}} \cdot z_{2}, y_{2}\}, \ldots, y_{n+1}\right\} \\
\text { ind. hyp. } & \sum_{s \in\{0,1\}^{n+1}} l^{\prime}(s) \cdot r^{\prime}(s)+l^{\prime \prime}(s) \cdot r^{\prime \prime}(s) \\
= & \sum_{s \in\{0,1\}^{n+1}} l(1, s) \cdot r(1, s)+l(0, s) \cdot r(0, s) \\
= & \sum_{s \in\{0,1\}^{n+2}} l(s) \cdot r(s),
\end{aligned}
$$

when the substitutions $\left\{z_{2}, y_{1}\right\}=: z_{2}^{\prime}$ and $\left\{z_{1}, y_{1}\right\}=: z_{1}^{\prime}$ to take advantage of Leibniz's rule as well as $z_{1}^{\prime}:=z_{1}, z_{2}^{\prime \prime}:=z_{2}$ and $y_{j}^{\prime}:=y_{j}^{\prime \prime}:=y_{j+1}$ for $i \in\{1, \ldots, n\}$ to arrange the indices into the form of equation (C.4) are made. 
Since universal enveloping algebras are Poisson algebras and $\hat{\mathfrak{h}}=\operatorname{ker} \delta$ is a subalgebra of $U\left(\mathfrak{g}_{h}\right)$, the lemma can be applied in our situation. Consider the form of the two products $F_{-1} F_{+2}+F_{+1} F_{-2}$ in equation (C.3). We can apply the above lemma C.0.1 to the two factors in which the quadratic $x$ occurs.

If this $x$ occurs in the left hand factor $F_{ \pm 1}$, then the degree of the right hand factor $F_{\mp 2}$ is $n+2$, greater than the degrees of $l(s)$ and $r(s)$, as well as of $[l(s), r(s)] \in \mathfrak{g}_{n+1}$ for all $s$. Therefore, there is no contribution in $h^{2}$ (at most one reordering in the quantization). However, due to reasons of degree, lead $L_{(1)}$ can only occur in a term quadratic in $h$.

Therefore only the products where the quadratic $x$ occurs in the right hand factor $F_{ \pm 2}$ can contribute. In other words, if one of $x_{-2}, x_{-1}, x_{0,-1}, \ldots, x_{0,-n}$ is quadratic, only the product $F_{+1} F_{-2}$ contributes, otherwise only $F_{-1} F_{+2}$ contributes.

Consider furthermore that since $L_{i}$ is obtained from $L_{-i}$ by changing the signs in the occurring words (this is the action of one the single generator of the Weyl group from lemma 2.2.17 for $d=3$ ), one can obtain the contribution to lead $L_{(1)}$ where one of the $x_{-2}, x_{-1}$, $x_{0,-1}, \ldots, x_{0,-n}$ is quadratic by calculating the corresponding contribution where one of the $x_{2}, x_{1}, x_{0,1}, \ldots, x_{0, n}$ is quadratic and changing all signs in the words.

We continue in the latter case, calculating the only product contributing to the summand in the expansion of (namely $F_{-1} F_{+2}$ ) that contributes to lead $L_{(1)}$. In the first equation, the fact that $F_{+2}$ can be expressed in the form of lemma C.0.1 is used (how exactly this is done for a given quadratic $x$ is listed below):

$$
\begin{aligned}
& F_{-1} \cdot F_{+2} \\
= & \sum_{s \in\{0,0.1\}^{n+1}} l(s) \cdot F_{-1} \cdot r(s)+h\left[l(s), F_{-1}\right] r(s) \\
= & \sum_{s \in\{0,1\}^{n+1}} l(s) \cdot r(s) \cdot F_{-1}+h l(s)\left[F_{-1}, r(s)\right]+h r(s)\left[l(s), F_{-1}\right]+h^{2}\left[\left[l(s), F_{-1}\right], r(s)\right] .
\end{aligned}
$$

Since the second and third summands on the right hand side of equation (C.6) are already in ascending order and the first one produces at most one commutator (from the reordering of $l$ and $r$ ), the only contribution to a term in $h^{2}$ can come from the last term. Because of this, we will only consider the last summand above.

The fact that the term $y:=-e(0-+)-e(0+-) \quad\left(=y_{i}\right.$ for all $\left.i \geq 2\right)$ appears frequently allows for a simplification:

$$
\begin{aligned}
& \sum_{s}\left[\left[l(s), F_{-1}\right], r(s)\right] \\
= & \sum_{s, s_{1}=0}\left[\left[l(s), F_{-1}\right], r(s)\right]+\sum_{s, s_{1}=1}\left[\left[l(s), F_{-1}\right], r(s)\right] \\
= & \sum_{s}\left[\left[\operatorname{ad}_{-y}^{\# 1 \text { in } s_{2}, \ldots s_{n+1}}\left[x_{1}, y_{1}\right], F_{-1}\right], \operatorname{ad}_{-y}^{\# 0 \text { in } s_{2} \ldots s_{n+1}} x_{2}\right]+\left[\operatorname{ad}_{-y}^{\# 1 \text { in } s_{2}, \ldots s_{n+1}} x_{1}, F_{-1}\right], \operatorname{ad}_{-y}^{\left.\# 0 \text { in } s_{2} \ldots s_{n+1}\left[x_{2}, y_{1}\right]\right]}
\end{aligned}
$$

$$
=\sum_{k=0}^{n}\left(\begin{array}{l}
n \\
k
\end{array}\right)\left[\left[\operatorname{ad}_{-y}^{n-k}\left[x_{1}, y_{1}\right], F_{-1}\right], \operatorname{ad}_{-y}^{k} x_{2}\right]+\quad\left[\left[\operatorname{ad}_{-y}^{n-k} x_{1}, F_{-1}\right], \operatorname{ad}_{-y}^{k}\left[x_{2}, y_{1}\right]\right]
$$


Because both lead $L_{(1)}$ and each remaining summand contain exactly $2 n+3$ zeroes each, all brackets where zeroes are contracted do not contribute to lead $L_{(1)}$. To calculate the contribution, we can therefore replace the Pohlmeyer-Rehren Lie bracket by the Lie bracket $[\cdot, \cdot]_{ \pm}$defined in proposition 1.8 .1 .

To streamline the contiuation of calculation (C.7), some more notation is helpful. Define the following symbols composed of binomial coefficients and Kronecker delta functions:

Definition C.0.2. Using the convention that $\left(\begin{array}{l}n \\ k\end{array}\right)=0$ if $n<0$, define:

$$
\begin{aligned}
f_{i, n, m} & :=\left(\begin{array}{c}
m+n-i \\
n
\end{array}\right)+\left(\begin{array}{c}
m+n-i \\
m
\end{array}\right)-(-1)^{n} \delta_{i, m}-(-1)^{m} \delta_{i, n} \\
& =h_{i, n, m}+h_{i, m, n} \\
& =\left(\begin{array}{c}
p-i \\
n
\end{array}\right)+\left(\begin{array}{c}
p-i \\
p-n
\end{array}\right)-(-1)^{n} \delta_{i, p-n}-(-1)^{p+n} \delta_{i, n} \\
& =\left(\begin{array}{c}
p-i \\
n
\end{array}\right)+\left(\begin{array}{c}
p-i \\
n-i
\end{array}\right)-(-1)^{n} \delta_{i, p-n}-(-1)^{p+n} \delta_{i, n}, \\
h_{i, n, m} & :=\left(\begin{array}{c}
m+n-i \\
n
\end{array}\right)-(-1)^{m} \delta_{i, n}, \\
v_{i, n, p}: & :(-1)^{n-p} h_{i, n, n-p} \\
& :=(-1)^{n-p}\left(\begin{array}{c}
p-i \\
n
\end{array}\right)+(-1)^{p+1} \delta_{i, p-n}, \\
u_{i, n, p} & :=(-1)^{n-p} f_{i, n, n-p} \\
& :=(-1)^{n-p}\left(\begin{array}{c}
p-i \\
n
\end{array}\right)+(-1)^{n-p}\left(\begin{array}{c}
p-i \\
p-n
\end{array}\right)+(-1)^{p+1} \delta_{i, p-n}-\delta_{i, n}, \\
U_{0, i, n, p}: & : \delta_{i, n}, \\
U_{k, i, n, p} & :=\sum_{l=0}^{p} U_{k-1, i, l, p+1} u_{l, n, p}, \\
V_{0, i, n, p}: & : \delta_{i, n}, \\
V_{k, i, n, p}: & : \sum_{l=0}^{p} U_{k-1, i, l, p+1} v_{l, n, p} .
\end{aligned}
$$

The above definitions imply the following statements:

- $(-1)^{n+p} \cdot v_{i, n, p} \geq 0$ and $v_{i, n, p}=0$ only if $i=p-n$ and $n$ even.

- $(-1)^{n+p} \cdot u_{i, n, p} \geq 0$ and $u_{i, n, p}=0$ only if $i=p-n=n$ and $n, p$ both even.

- $u_{i, n, p}=0$ if $i>\max (n, p-n)$ because all summands vanish in this case.

- $u_{i, p-n, p}=(-1)^{p} u_{i, n, p}$.

- $v_{i, p-n, p}=(-1)^{p}\left(u_{i, n, p}-v_{i, n, p}\right)$.

- $U_{k, i, p-n, p}=(-1)^{p} U_{k, i, n, p}$. 
The notation just introduced allows to fully take advantage of the simpler Lie bracket $[\cdot, \cdot]_{ \pm}$and to concisely formulate the individual steps needed in the continuation of calculation (C.7).

Lemma C.0.3. The following formulas for special terms hold in ${ }_{ \pm} \mathfrak{g}$ :

1.

$$
\operatorname{ad}_{-y} e\left(0^{m}+\right)=-\alpha \cdot e\left(0^{m+1}+\right) .
$$

2.

$$
\operatorname{ad}_{-y}^{p} e\left(0^{m}+\right)=(-\alpha)^{p} e\left(0^{m+p}+\right)
$$

3.

$$
\begin{aligned}
& \operatorname{ad}_{-y} e\left(-0^{n}-0^{p-n}-\right) \\
= & \alpha \sum_{i=0}^{p} u_{i, n, p} e\left(-0^{i}-0^{p+1-i}-\right) \\
= & \alpha \sum_{i=0}^{p} U_{1, i, n, p} e\left(-0^{i}-0^{p+1-i}-\right) .
\end{aligned}
$$

4.

$$
\operatorname{ad}_{-y}^{r} e\left(-0^{n}-0^{p-n}-\right)=\alpha^{r} \sum_{i=0}^{p+r} U_{r, i, n, p} e\left(-0^{i}-0^{p+r-i}-\right)
$$

5.

$$
\begin{aligned}
& {\left[e\left(0^{r}+\right), e\left(-0^{n}-0^{p-n}-\right)\right]_{ \pm} } \\
= & \alpha\left((-1)^{r+1} \sum_{i=0}^{\max (n, p-n)} u_{i, n, p} e\left(-0^{i}-0^{p+r-i}\right)\right) .
\end{aligned}
$$

6.

$$
\begin{aligned}
& {\left[e\left(-0^{n}-0^{m}\right), e(0++)\right]_{ \pm} } \\
= & \alpha\left((-1)^{m} \sum_{i=0}^{m}\left(\left(\begin{array}{c}
n+m-i \\
n
\end{array}\right)-(-1)^{n} \delta_{i, m}\right) e\left(0^{i}-0^{m+n-i+1}+\right)\right) .
\end{aligned}
$$

7. The above is a special case of

$$
\begin{aligned}
& {\left[e\left(-0^{n}-0^{p-n}\right), e\left(+0^{m}+0^{q-m}\right)\right]_{ \pm} } \\
= & \alpha(-1)^{q} \sum_{i=0}^{p-n} \sum_{j=0}^{q-m} v_{i, n, p} v_{j, m, q} e\left(0^{i}-0^{p+q-i-j}+0^{j}\right) .
\end{aligned}
$$


8.

$$
e\left(0^{q}-0^{l-q-p}+0^{p}\right)=(-1)^{p} \sum_{i=0}^{p}\left(\begin{array}{c}
q+i \\
i
\end{array}\right)\left(\begin{array}{c}
l-q-i \\
p-i
\end{array}\right) e\left(0^{q+i}-0^{l-q-i}+\right)
$$

9.

$$
e\left(0^{q}+0^{l-q-p}-0^{p}\right)=(-1)^{l+q+1} \sum_{i=0}^{q}\left(\begin{array}{c}
p+i \\
i
\end{array}\right)\left(\begin{array}{c}
l-p-i \\
q-i
\end{array}\right) e\left(0^{p+i}-0^{l-p-i}+\right) .
$$

Proof. $\quad 1$.

$$
\begin{aligned}
\operatorname{ad}_{-y} e\left(0^{m}+\right) & =-\left[e\left(0^{m}+\right), e(0-+)+e(0+-)\right]_{ \pm} \\
& =-\alpha\left((-1)^{m+1+m+1+2} e\left(0^{m}{ }_{0}^{+}\right)+(-1)^{m+1+m+1+3} e\left(0^{m}+0\right)\right) \\
& =-\alpha \cdot e\left(0^{m+1}+\right)
\end{aligned}
$$

2. Induction over $p$ using 1 .

3.

$$
\begin{aligned}
& \operatorname{ad}_{-y} e\left(-0^{n}-0^{p-n}-\right) \\
= & -\left[e\left(-0^{n}-0^{p-n}-\right), e(0-+)+e(0+-)\right]_{ \pm} \\
= & -\alpha\left((-1)^{p} e\left(-0^{p-n}-0^{n+1}-\right)+(-1)^{p+n+1} e\left(\left[\begin{array}{c}
-0^{n} \\
-0^{p-n}
\end{array}\right] 0-\right)+e\left(-0^{n}-0^{p-n+1}-\right)\right) \\
= & -\alpha\left((-1)^{p} e\left(-0^{p-n}-0^{n+1}-\right)+(-1)^{p+n+1}\left(e\left(-\left[\begin{array}{c}
0^{n} \\
-0^{p-n}
\end{array}\right]-\right)+e\left(-\left[\begin{array}{c}
-0^{n} \\
0^{p-n}
\end{array} 0-\right)\right)+e\left(-0^{n}-0^{p-n+1}-\right)\right)\right. \\
= & -\alpha\left((-1)^{p} e\left(-0^{p-n}-0^{n+1}-\right)+(-1)^{p+n+1}\left(\sum_{i=0}^{n}\left(\begin{array}{c}
p-i \\
n-i
\end{array}\right) e\left(-0^{i}-0^{p-i} 0-\right)\right.\right. \\
& \left.\left.+\sum_{i=0}^{p-n}\left(\begin{array}{c}
p-i \\
p-n-i
\end{array}\right) e\left(-0^{i}-0^{p-i} 0-\right)\right)+e\left(-0^{n}-0^{p-n+1}-\right)\right) \\
= & \alpha \sum_{i=0}^{p}\left((-1)^{p+1} \delta_{i, p-n}+(-1)^{p+n}\left(\left(\begin{array}{c}
p-i \\
n-i
\end{array}\right)+\left(\begin{array}{c}
p-i \\
n
\end{array}\right)\right)-\delta_{i, n}\right) e\left(-0^{i}-0^{p+1-i}-\right) \\
= & \alpha \sum_{i=0}^{p} u_{i, n, p} e\left(-0^{i}-0^{p+1-i}-\right) \\
= & \alpha \sum_{i=0}^{p} U_{1, i, n, p} e\left(-0^{i}-0^{p+1-i}-\right) .
\end{aligned}
$$

4. Induction over $r$. Base case $r=0$ :

$$
\operatorname{ad}_{-y}^{0} e\left(-0^{n}-0^{p-n}-\right)=e\left(-0^{n}-0^{p-n}-\right)=\sum_{i=0}^{p} \delta_{i, n} e\left(-0^{i}-0^{p-i}-\right)=\alpha^{0} \sum_{i=0}^{p} U_{0, i, n, p} e\left(-0^{i}-0^{p-i}-\right) .
$$




\section{Inductive step:}

$$
\begin{aligned}
& \operatorname{ad}_{-y}^{r+1} e\left(-0^{n}-0^{p-n}-\right) \\
& \underline{\underline{\underline{3}}} \operatorname{ad}_{-y}^{r} \alpha \sum_{j=0}^{p} u_{j, n, p} e\left(-0^{j}-0^{p+1-j}-\right) \\
& \stackrel{\text { ind.hyp. }}{=} \alpha^{r+1} \sum_{j=0}^{p} \sum_{i=0}^{(p+1)+r} U_{r, i, j, p+1} u_{j, n, p} e\left(-0^{i}-0^{p+r+1-i}-\right) \\
& =\alpha^{r+1} \sum_{i=0}^{p+(r+1)} \underbrace{\sum_{j=0}^{p} U_{r, i, j, p+1} u_{j, n, p}}_{) U_{r+1, i, n, p}} e\left(-0^{i}-0^{p+(r+1)-i}-\right) .
\end{aligned}
$$

5.

$$
\begin{aligned}
& {\left[e\left(0^{r}+\right), e\left(-0^{n}-0^{p-n}-\right)\right]_{ \pm} } \\
= & -\left[e\left(-0^{n}-0^{p-n}-\right), e\left(0^{r}+\right)\right]_{ \pm} \\
= & -\alpha\left((-1)^{p+r+1} e\left(-0^{p-n}-0^{n+r}\right)+(-1)^{p+n+r} e\left(\sum_{-0^{p-n}}^{-0^{n}} 0^{r}\right)+(-1)^{r+1} e\left(-0^{n}-0^{p-n+r}\right)\right. \\
= & \alpha\left((-1)^{r+1} \sum_{i=0}^{\max (n, p-n)} u_{i, n, p} e\left(-0^{i}-0^{p+r-i}\right)\right) .
\end{aligned}
$$

6.

$$
\begin{aligned}
& {\left[e\left(-0^{n}-0^{m}\right), e(0++)\right]_{ \pm} } \\
= & \alpha\left((-1)^{n+m+1} e\left(0^{m}-0^{n+1}+\right)+(-1)^{m+2} e\left(\left[\begin{array}{c}
-0^{n} \\
0^{m}
\end{array} 0+\right)\right)\right. \\
= & \alpha\left((-1)^{n+m+1} e\left(0^{m}-0^{n+1}+\right)+(-1)^{m} \sum_{i=0}^{m}\left(\begin{array}{c}
n+m-i \\
n
\end{array}\right) e\left(0^{i}-0^{m+n-i+1}+\right)\right) \\
= & \alpha\left((-1)^{m} \sum_{i=0}^{m}\left(\left(\begin{array}{c}
n+m-i \\
n
\end{array}\right)-(-1)^{n} \delta_{i, m}\right) e\left(0^{i}-0^{m+n-i+1}+\right)\right) .
\end{aligned}
$$


7.

$$
\begin{aligned}
& {\left[e\left(-0^{n}-0^{p-n}\right), e\left(+0^{m}+0^{q-m}\right)\right]_{ \pm}} \\
& =\alpha\left((-1)^{p} e\left(0^{p-n}-0^{n+m}+0^{q-m}\right)+(-1)^{p+m+1} e\left(0^{p-n}-0^{n}\left(\begin{array}{c}
0^{m}+ \\
0^{q-m}
\end{array}\right)\right.\right. \\
& +(-1)^{p+n+1} e\left(\begin{array}{c|c}
-0^{n} \\
0^{p-n}
\end{array} 0^{m}+0^{q-m}\right)+(-1)^{p+n+m} e\left(\begin{array}{c|c}
-0^{n} & 0^{m}+ \\
0^{p-n} & 0^{q-m}
\end{array}\right) \\
& =\alpha(-1)^{q}\left((-1)^{p+q} e\left(0^{p-n}-0^{n+m}+0^{q-m}\right)+(-1)^{p+m+1+q} \sum_{j=0}^{q-m}\left(\begin{array}{c}
q-j \\
m
\end{array}\right) e\left(0^{p-n}-0^{p+q-j}+0^{j}\right)\right. \\
& +(-1)^{p+n+1+q} \sum_{i=0}^{p-n}\left(\begin{array}{c}
p-i \\
n
\end{array}\right) e\left(0^{i}-0^{p+q-i}+0^{q}\right) \\
& \left.-(-1)^{p+n+m+q} \sum_{i=0}^{p-n} \sum_{j=0}^{q-m}\left(\begin{array}{c}
p-i \\
n
\end{array}\right)\left(\begin{array}{c}
q-j \\
m
\end{array}\right) e\left(0^{i}-0^{p+q-i-j}+0^{j}\right)\right) \\
& =\alpha(-1)^{q} \sum_{i=0}^{p-n} \sum_{j=0}^{q-m}\left((-1)^{p+q} \delta_{i, p-n} \delta_{j, q-m}\right. \\
& +(-1)^{p+m+1+q} \delta_{i, p-n}\left(\begin{array}{c}
q-j \\
m
\end{array}\right)+(-1)^{p+n+1+q} \delta_{j, q-m}\left(\begin{array}{c}
p-i \\
n
\end{array}\right) \\
& \left.-(-1)^{p+n+m+q}\left(\begin{array}{c}
p-i \\
n
\end{array}\right)\left(\begin{array}{c}
q-j \\
m
\end{array}\right)\right) e\left(0^{i}-0^{p+q-i-j}+0^{j}\right) \\
& =\alpha(-1)^{q} \sum_{i=0}^{p-n} \sum_{j=0}^{q-m}\left((-1)^{p} \delta_{i, p-n}+(-1)^{p+n+1}\left(\begin{array}{c}
p-i \\
n
\end{array}\right)\right) \\
& \cdot\left((-1)^{q} \delta_{j, q-m}+(-1)^{q+m+1}\left(\begin{array}{c}
q-j \\
m
\end{array}\right)\right) e\left(0^{i}-0^{p+q-i-j}+0^{j}\right) \\
& =\alpha(-1)^{q} \sum_{i=0}^{p-n} \sum_{j=0}^{q-m} v_{i, n, p} v_{j, m, q} e\left(0^{i}-0^{p+q-i-j}+0^{j}\right) \text {. }
\end{aligned}
$$

8.

$$
e\left(0^{q}-0^{l-q-p}+0^{p}\right) \stackrel{\frac{1.2 .5}{=}}{=}(-1)^{l+2-(l-p)} e\left(\begin{array}{c}
0^{q-0^{l-q-p}} \\
0^{p}
\end{array}+\right)=(-1)^{p} \sum_{i=0}^{p}\left(\begin{array}{c}
q+i \\
i
\end{array}\right)\left(\begin{array}{c}
l-q-i \\
p-i
\end{array}\right) e\left(0^{q+i}-0^{l-q-i}+\right) .
$$

9.

$$
e\left(0^{q}+0^{l-q-p}-0^{p}\right) \stackrel{1.2 .5}{=}(-1)^{l+3} e\left(0^{p}-0^{l-q-p}+0^{q}\right)=(-1)^{l+q+1} \sum_{i=0}^{q}\left(\begin{array}{c}
p+i \\
i
\end{array}\right)\left(\begin{array}{c}
l-p-i \\
q-i
\end{array}\right) e\left(0^{p+i}-0^{l-p-i}+\right) .
$$


The above lemma can be used to give a concrete formula for $F_{-1}$ (recall that we are in the case where no quadratic $x$ occurs here):

$$
\begin{aligned}
F_{-1} & =\operatorname{ad}_{-y}^{n}[2 e(0--),-\sqrt{2} e(+--)]_{ \pm} \\
& =2 \sqrt{2} \alpha \operatorname{ad}_{-y}^{n} e(--0-) \\
& \stackrel{\text { C.19 }}{=} 2 \sqrt{2} \alpha^{n+1} \sum_{i=0}^{n} U_{n, i, 0,1} e\left(-0^{i}-0^{n+1-i}-\right) .
\end{aligned}
$$

We now continue the discussion of the summands, taking into consideration which one of the $x$ is quadratic.

- If $x_{2}$ is quadratic, then use $y_{1}=-\sqrt{2} e(-++), z_{1}=-e(0+), z_{2}=e(0+)$. Note that $[e(0+), e(-++)]_{ \pm}=-\alpha e(0++)$. We continue calculation (C.7):

$$
\begin{aligned}
& \sum_{k=0}^{n}\left(\begin{array}{l}
n \\
k
\end{array}\right)\left(\left[[\operatorname{ad}_{-y}^{n-k} \underbrace{\left[x_{1}, y_{1}\right]_{ \pm}}_{=-\sqrt{2} \alpha e(0++)}, F_{-1}]_{ \pm}, \operatorname{ad}_{-y}^{k} x_{2}\right]_{ \pm}+[\left[\operatorname{ad}_{-y}^{n-k} x_{1}, F_{-1}\right]_{ \pm}, \operatorname{ad}_{-y}^{k} \underbrace{\left[x_{2}, y_{1}\right]_{ \pm}}_{=\sqrt{2} \alpha e(0++)}]_{ \pm}\right) \\
& =-\sqrt{2} \alpha \sum_{k=0}^{n}\left(\begin{array}{l}
n \\
k
\end{array}\right)\left(\left[\left[\operatorname{ad}_{-y}^{n-k} e(0++), F_{-1}\right]_{ \pm}, \operatorname{ad}_{-y}^{k} e(0+)\right]_{ \pm}+\left[\left[\operatorname{ad}_{-y}^{n-k} e(0+), F_{-1}\right]_{ \pm}, \operatorname{ad}_{-y}^{k} e(0++)\right]_{ \pm}\right) \\
& \stackrel{\text { Jacobi }}{=}-\sqrt{2} \alpha \sum_{k=0}^{n}\left(\begin{array}{l}
n \\
k
\end{array}\right)\left([\left[\operatorname{ad}_{-y}^{k} e(0+), F_{-1}\right]_{ \pm}, \underbrace{\operatorname{ad}_{-y}^{n-k} e(0++)}_{=0 \text { if } n-k>0}]_{ \pm}+[F_{-1}, \underbrace{\left[\operatorname{ad}_{-y}^{n-k} e(0+), \operatorname{ad}_{-y}^{k} e(0++)\right]_{ \pm}}_{=0}]_{ \pm}\right. \\
& \left.+[\left[\operatorname{ad}_{-y}^{n-k} e(0+), F_{-1}\right]_{=}, \underbrace{\operatorname{ad}_{-y}^{k} e(0++)}_{=0 \text { if } k>0}]_{ \pm}\right) \\
& =-2 \sqrt{2} \alpha\left[\left[\operatorname{ad}_{-y}^{n} e(0+), F_{-1}\right]_{ \pm}, e(0++)\right]_{ \pm} \\
& \stackrel{\text { C. } 17}{=} 2 \sqrt{2}(-\alpha)^{n+1}\left[\left[e\left(0^{n+1}+\right), F_{-1}\right]_{ \pm}, e(0++)\right]_{ \pm} \\
& \stackrel{C .25}{=} 8(-1)^{n+1} \alpha^{2 n+2} \sum_{i=0}^{n} U_{n, i, 0,1}\left[\left[e\left(0^{n+1}+\right), e\left(-0^{i}-0^{n+1-i}-\right)\right]_{ \pm}, e(0++)\right]_{ \pm} \\
& \stackrel{\text { C.20 }}{-} 8(-1)^{2 n+3} \alpha^{2 n+3} \sum_{i=0}^{n} \sum_{j=0}^{n+1} U_{n, i, 0,1} u_{j, i, n+1}\left[e\left(-0^{j}-0^{2 n+2-j}\right), e(0++)\right]_{ \pm} \\
& \stackrel{\text { C.21 }}{=}-8 \alpha^{2 n+4} \sum_{i=0}^{n} \sum_{j=0}^{n+1} \sum_{K=0}^{2 n+2-j} U_{n, i, 0,1} u_{j, i, n+1} v_{K, j, 2 n+2} e\left(0^{K}-0^{2 n+3-K}+\right) \text {. }
\end{aligned}
$$

- $x_{-2}$ is quadratic. The contribution to lead $L_{(1)}$ is the same as for the case of $x_{2}$ being quadratic, except of a sign change in the occurring words:

$$
-8 \alpha^{2 n+4} \sum_{i=0}^{n} \sum_{j=0}^{n+1} \sum_{K=0}^{2 n+2-j} U_{n, i, 0,1} u_{j, i, n+1} v_{K, j, 2 n+2} e\left(0^{K}+0^{2 n+3-K}-\right) .
$$


One can now use equation (C.24) to develop the term (C.27) into Euler elements whose words end with $\mathrm{a}+$ and then merge the total contribution of the summands with quadratic $x_{ \pm 2}$ into the term

$$
-8 \alpha^{2 n+4} \sum_{i=0}^{n} \sum_{j=0}^{n+1} \sum_{K=0}^{2 n+2-j} \sum_{L=0}^{2 n+3} U_{n, i, 0,1} u_{j, i, n+1} v_{K, j, 2 n+2} v_{L, 2 n+3-K, 2 n+3} e\left(0^{L}-0^{2 n+3-L}+\right) .
$$

- $x_{1}$ is quadratic. We can use antisymmetry to obtain the form of lemma C.0.1, acquiring an additional sign for $y_{1}$. Then, $y_{1}=-2 e(0++)$, and we have two summands, with $z_{11}=z_{22}=\frac{1}{\sqrt{2}} e(0+), z_{21}=z_{12}=e(-+)$ respectively. Note $\left[y_{1}, e(0+)\right]_{ \pm}=0$.

$$
\begin{gathered}
\sum_{k=0}^{n}\left(\begin{array}{l}
n \\
k
\end{array}\right)\left(\left[[\operatorname{ad}_{-y}^{n-k} \underbrace{\left[x_{11}, y_{1}\right]_{ \pm}}_{=0}, F_{-1}]_{ \pm}, \operatorname{ad}_{-y}^{k} x_{21}\right]_{ \pm}+[\left[\operatorname{ad}_{-y}^{n-k} x_{11}, F_{-1}\right]_{ \pm}, \operatorname{ad}_{-y}^{k} \underbrace{\left[x_{21}, y_{1}\right]_{ \pm}}_{=-4 \alpha e(0++)}]_{ \pm}\right. \\
\left.+\left[[\operatorname{ad}_{-y}^{n-k} \underbrace{\left[x_{12}, y_{1}\right]_{ \pm}}_{=-4 \alpha e(0++)}, F_{-1}]_{ \pm}, \operatorname{ad}_{-y}^{k} x_{22}\right]_{ \pm}+[\left[\operatorname{ad}_{-y}^{n-k} x_{12}, F_{-1}\right]_{ \pm}, \operatorname{ad}_{-y}^{k} \underbrace{\left[x_{22}, y_{1}\right]_{ \pm}}_{=0}]_{ \pm}\right)
\end{gathered}
$$

$=-2 \sqrt{2} \alpha \sum_{k=0}^{n}\left(\begin{array}{l}n \\ k\end{array}\right)\left(\left[\left[\operatorname{ad}_{-y}^{n-k} e(0+), F_{-1}\right]_{ \pm}, \operatorname{ad}_{-y}^{k} e(0++)\right]_{ \pm}+\left[\left[\operatorname{ad}_{-y}^{n-k} e(0++), F_{-1}\right]_{ \pm}, \operatorname{ad}_{-y}^{k} e(0+)\right]_{ \pm}\right)$.

From here on, we can use the above calculation (C.26) for the $x_{2}$ case, noting that the term on the right hand of (C.29) side occurs (with a factor of 2) in the second line of calculation (C.26).

- For quadratic $x_{-1}$, we can use the same sign change trick as for quadratic $x_{-2}$.

- $x_{0, \pm i}$ is quadratic, $i=1, \ldots, n$. Again, use antisymmetry to obtain the form of lemma C.0.1. acquiring a sign for $y_{1}$. Then,

$$
\begin{aligned}
y_{1} & =\operatorname{ad}_{-y}^{i-1}[e(0++),-\sqrt{2} e(-++)]_{ \pm} \\
& =3 \sqrt{2} \alpha \operatorname{ad}_{-y}^{i-1} e(++0+)
\end{aligned}
$$

and again we have two summands, with $z_{1}=\frac{1}{2} e(0+), z_{2}=e(0-)$ and $z_{1}=\frac{1}{2} e(0-)$, $z_{2}=e(0+)$ respectively. Further, $y_{2}=\ldots=y_{n-i}=y$. Again, $\left[y_{1}, e(0+)\right]_{ \pm}=0$. One can now perform a similar calculation to the above cases which are omitted here because they require even more arduous notation than calculation (C.26).

Note that the right hand side of equation (C.26) as well as the other summands are linear combination of the Euler elements $e\left(0^{L}-0^{2 n+3-L}+\right)$ with $L \in\{0, \ldots, 2 n+3\}$. Since these Euler elements are linearly independent, to prove that lead $L_{(\mathbb{D})}$ occurs in the quantum correction, one only has to prove that the total coefficient (from all the summands of the expansion of (C.2)) for $L=0$ is nonzero.

This coefficient has been calculated using computer algebra for $l=3,5,7,9$ both by explicitly calculating the full quantum correction of term (C.1) using the Meusburger-Rehren approach with computer algebra and by using the simplifications discussed in this appendix. For each $l$, the coefficients agree and are in fact nonzero. 
Given the construction of the symbols that make up the coefficient, the approach to a general proof that the coefficient is nonzero suggesting itself is to exploit relations of binomial coefficients and Kronecker deltas. Unfortunately, the problem has resisted this kind of attack so far. 
Appendix C. Generating Leading Terms of Exceptional Elements in $\hat{\mathfrak{h}}$ 


\section{Appendix D}

\section{A Pseudo-Hall-Basis up to $l=4$ of $\mathfrak{g}$ for $d=3$}

This appendix gives a pseudo-Hall-basis of the Pohlmeyer-Rehren Lie algebra up to degree $l=4$ for $d=3$, computed by using the algorithm PHallSecondGrad given in subsection 4.5.1 and implemented by Mathematica module PHallSecondGrad documented in section A.3.4. We use the initial set

$$
\begin{aligned}
X:=\quad\{\mathrm{x} & =x=e(0+) \\
\mathrm{v} & ={ }^{2} v^{-2}=e(0--) \\
\mathrm{w} & \left.={ }^{1} v^{-1}=e(00-)+e(--+)\right\}
\end{aligned}
$$

of elements from remark 2.3.17. To be precise, a pseudo-Hall-basis of the subalgebra generated by these elements is computed. The orders used are $<_{H}=<_{S}=$ DegLex with $x<v<w$. The specific code to generate this table is

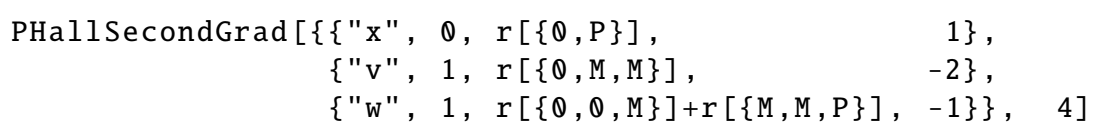

The table was reformatted automatically into $\mathrm{AT}_{\mathrm{E}} \mathrm{X}$ code with Mathematica, using the shorthand $x^{n} \cdot g:=\operatorname{ad}_{x}^{n} g$ for $g \in \mathfrak{g}$. In spite of being slightly misleading (no element $x^{n}$ operates on $\mathrm{g})$, this notation is used because it is easier to read than the correct notation $(x \text {. })^{n} g$. Fractions occurring in B are automatically expanded so that all coefficients are integers.

By comparison of dimensions (the Mathematica module PHallSecondGrad automatically outputs the dimension of each stratum), we can conclude that for $l=1, \ldots, 9$, the pseudoHall-basis of the Lie algebra generated by $x, v, w$ is also a pseudo-Hall-basis of $\langle x\rangle \oplus \mathfrak{g}_{\geq 1}$ up to $l=9$.

\section{Tuple H of basis vectors}

\begin{tabular}{|c|r|c|c|}
\hline 1 & $\mathrm{~m}$ & $h \in \mathrm{H}$ in terms of $\mathrm{X}$ & $h \in \mathrm{H}$ in terms of the Euler-Lyndon basis \\
\hline 0 & 1 & $x$ & $\mathrm{e}(0+)$ \\
\hline 1 & -2 & $v$ & $\mathrm{e}(0--)$ \\
\hline \hline
\end{tabular}


Appendix D. A Pseudo-Hall basis of $\mathfrak{g}$ for $d=3$

\begin{tabular}{|c|c|c|c|}
\hline 1 & $\mathrm{~m}$ & $h \in \mathrm{H}$ in terms of $\mathrm{X}$ & $h \in \mathrm{H}$ in terms of the Euler-Lyndon basis \\
\hline 1 & $\overline{-1}$ & $w$ & $\mathrm{e}(00-)+\mathrm{e}(--+)$ \\
\hline 1 & -1 & $x . v$ & $\mathrm{e}(00-)-\mathrm{e}(--+)$ \\
\hline 1 & 0 & $x . w$ & $\mathrm{e}(0-+)+\mathrm{e}(0+-)$ \\
\hline 1 & 0 & $x^{2} . v$ & $\mathrm{e}(0-+)-\mathrm{e}(0+-)$ \\
\hline 1 & 1 & $x^{2} \cdot w$ & $\mathrm{e}(00+)+\mathrm{e}(-++)$ \\
\hline 1 & 1 & $x^{3} \cdot v$ & $\mathrm{e}(00+)-\mathrm{e}(-++)$ \\
\hline 1 & 2 & $x^{4} . v$ & $\mathrm{e}(0++)$ \\
\hline 2 & -3 & $v . w$ & $\mathrm{e}(0---)$ \\
\hline 2 & -2 & v.x.w & $\mathrm{e}(---+)$ \\
\hline 2 & -2 & $v \cdot x^{2} \cdot v$ & $\mathrm{e}(00--)+\mathrm{e}(---+)$ \\
\hline 2 & -1 & $v \cdot x^{2} \cdot w$ & $\mathrm{e}(000-)-2 \mathrm{e}(0--+)-\mathrm{e}(0-+-)-\mathrm{e}(0+--)$ \\
\hline 2 & -1 & $v \cdot x^{3} \cdot v$ & $\mathrm{e}(000-)+2 \mathrm{e}(0--+)+\mathrm{e}(0-+-)-\mathrm{e}(0+--)$ \\
\hline 2 & -1 & $w . x . w$ & $\mathrm{e}(000-)-\mathrm{e}(0-+-)-\mathrm{e}(0+--)$ \\
\hline 2 & -1 & $w \cdot x^{2} \cdot v$ & $5 e(000-)+e(0-+-)+e(0+--)$ \\
\hline 2 & 0 & $v \cdot x^{4} . v$ & $\mathrm{e}(00-+)+\mathrm{e}(00+-)+\mathrm{e}(0-0+)+\mathrm{e}(--++)$ \\
\hline 2 & 0 & $w \cdot x^{2} \cdot w$ & $\mathrm{e}(00-+)+\mathrm{e}(00+-)+\mathrm{e}(0-0+)-\mathrm{e}(--++)$ \\
\hline 2 & 0 & $w \cdot x^{3} \cdot v$ & $3 \mathrm{e}(00-+)+\mathrm{e}(00+-)+\mathrm{e}(0-0+)+\mathrm{e}(--++)$ \\
\hline 2 & 0 & {$\left[x . v, x^{2} . w\right]$} & $\mathrm{e}(00-+)-\mathrm{e}(00+-)+\mathrm{e}(0-0+)+\mathrm{e}(--++)$ \\
\hline 2 & 1 & $w \cdot x^{4} \cdot v$ & $\mathrm{e}(000+)-\mathrm{e}(0-++)-\mathrm{e}(0+-+)-2 \mathrm{e}(0++-)$ \\
\hline 2 & 1 & {$\left[x . v, x^{4} . v\right]$} & $\mathrm{e}(000+)-\mathrm{e}(0-++)+\mathrm{e}(0+-+)+2 \mathrm{e}(0++-)$ \\
\hline 2 & 1 & {$\left[x . w, x^{2} . w\right]$} & $\mathrm{e}(000+)-\mathrm{e}(0-++)-\mathrm{e}(0+-+)$ \\
\hline 2 & 1 & {$\left[x . w, x^{3} . v\right]$} & $\mathrm{e}(000+)+\mathrm{e}(0-++)-\mathrm{e}(0+-+)-2 \mathrm{e}(0++-)$ \\
\hline 2 & 2 & {$\left[x . w, x^{4} . v\right]$} & $\mathrm{e}(-+++)$ \\
\hline 2 & 2 & {$\left[x^{2} . v, x^{4} . v\right]$} & $\mathrm{e}(00++)-\mathrm{e}(-+++)$ \\
\hline 2 & 3 & {$\left[x^{2} . w, x^{4} . v\right]$} & $\mathrm{e}(0+++)$ \\
\hline 3 & -4 & $v . v \cdot x . w$ & $\mathrm{e}(0----)$ \\
\hline 3 & -3 & $v \cdot v \cdot x^{2} \cdot w$ & $2 \mathrm{e}(00---)+\mathrm{e}(0-0--)+\mathrm{e}(----+)$ \\
\hline 3 & -3 & $v \cdot v \cdot x^{3} \cdot v$ & $4 \mathrm{e}(00---)+\mathrm{e}(0-0--)-2 \mathrm{e}(----+)$ \\
\hline 3 & -3 & $w . v \cdot x . w$ & $\mathrm{e}(00---)-2 \mathrm{e}(----+)$ \\
\hline 3 & -2 & $v \cdot v \cdot x^{4} \cdot v$ & $\begin{array}{c}2 \mathrm{e}(000--)+\mathrm{e}(00-0-)-2 \mathrm{e}(0---+)-2 \mathrm{e}(0--+-)-\mathrm{e}(0-+--)+ \\
\mathrm{e}(0+---)\end{array}$ \\
\hline 3 & -2 & $w \cdot v \cdot x^{2} \cdot w$ & $3 \mathrm{e}(000--)+\mathrm{e}(00-0-)-2 \mathrm{e}(0---+)-\mathrm{e}(0--+-)$ \\
\hline 3 & -2 & $w \cdot v \cdot x^{3} \cdot v$ & $3 \mathrm{e}(000--)+2 \mathrm{e}(0---+)+\mathrm{e}(0--+-)+\mathrm{e}(0-+--)$ \\
\hline 3 & -2 & $w \cdot w \cdot x^{2} \cdot v$ & $4 \mathrm{e}(000--)+\mathrm{e}(00-0-)$ \\
\hline 3 & -2 & {$\left[x . v, v \cdot x^{2} \cdot w\right]$} & $2 \mathrm{e}(000--)+\mathrm{e}(00-0-)+4 \mathrm{e}(0---+)+2 \mathrm{e}(0--+-)+\mathrm{e}(0-+--)$ \\
\hline 3 & -2 & {$[x . v, w . x . w]$} & $\mathrm{e}(000--)-\mathrm{e}(0--+-)-\mathrm{e}(0-+--)$ \\
\hline 3 & -1 & $w \cdot v \cdot x^{4} \cdot v$ & $\begin{array}{c}\mathrm{e}(0000-)+4 \mathrm{e}(00--+)+3 \mathrm{e}(00-+-)+\mathrm{e}(00+--)+\mathrm{e}(0-0-+)+ \\
\mathrm{e}(0-0+-)-3 \mathrm{e}(---++)-\mathrm{e}(--+-+)\end{array}$ \\
\hline 3 & -1 & $w \cdot w \cdot x^{2} \cdot w$ & $\begin{array}{c}\mathrm{e}(0000-)+2 \mathrm{e}(00--+)+\mathrm{e}(00-+-)+\mathrm{e}(00+--)+\mathrm{e}(0-0-+)+ \\
\mathrm{e}(0-0+-)+3 \mathrm{e}(---++)+\mathrm{e}(--+-+)\end{array}$ \\
\hline 3 & -1 & $w \cdot w \cdot x^{3} \cdot v$ & $\begin{array}{c}11 \mathrm{e}(0000-)-6 \mathrm{e}(00--+)-3 \mathrm{e}(00-+-)-\mathrm{e}(00+--)-3 \mathrm{e}(0-0-+)- \\
\mathrm{e}(0-0+-)+3 \mathrm{e}(---++)+\mathrm{e}(--+-+)\end{array}$ \\
\hline 3 & -1 & {$\left[x . v, v . x^{4} . v\right]$} & $\begin{array}{c}\mathrm{e}(0000-)+6 \mathrm{e}(00--+)+3 \mathrm{e}(00-+-)-\mathrm{e}(00+--)+3 \mathrm{e}(0-0-+)+ \\
\mathrm{e}(0-0+-)+2 \mathrm{e}(0--0+)+3 \mathrm{e}(---++)+\mathrm{e}(--+-+)\end{array}$ \\
\hline 3 & -1 & {$\left[x . v, w \cdot x^{2} . w\right]$} & $\begin{array}{c}\mathrm{e}(0000-)+4 \mathrm{e}(00--+)+\mathrm{e}(00-+-)-\mathrm{e}(00+--)+3 \mathrm{e}(0-0-+)+ \\
\mathrm{e}(0-0+-)+2 \mathrm{e}(0--0+)-3 \mathrm{e}(---++)-\mathrm{e}(--+-+)\end{array}$ \\
\hline 3 & -1 & {$\left[x . v, w . x^{3} . v\right]$} & $\begin{array}{c}11 \mathrm{e}(0000-)-12 \mathrm{e}(00--+)-3 \mathrm{e}(00-+-)+\mathrm{e}(00+--)-5 \mathrm{e}(0-0-+)- \\
\mathrm{e}(0-0+-)-2 \mathrm{e}(0--0+)-3 \mathrm{e}(---++)-\mathrm{e}(--+-+)\end{array}$ \\
\hline 3 & -1 & {$\left[x . v,\left[x . v, x^{2} . w\right]\right]$} & $\begin{array}{c}5 \mathrm{e}(0000-)-6 \mathrm{e}(00--+)+\mathrm{e}(00-+-)-\mathrm{e}(00+--)-3 \mathrm{e}(0-0-+)+ \\
\mathrm{e}(0-0+-)-2 \mathrm{e}(0--0+)-3 \mathrm{e}(---++)-\mathrm{e}(--+-+)\end{array}$ \\
\hline 3 & -1 & {$\left[x . w, v \cdot x^{2} . w\right]$} & $\begin{array}{c}\mathrm{e}(0000-)-2 \mathrm{e}(00--+)-\mathrm{e}(00-+-)+\mathrm{e}(00+--)-\mathrm{e}(0-0-+)- \\
\mathrm{e}(0-0+-)-2 \mathrm{e}(0--0+)-\mathrm{e}(---++)-\mathrm{e}(--+-+)\end{array}$ \\
\hline 3 & -1 & {$\left[x . w, v \cdot x^{3} . v\right]$} & $\begin{array}{c}\mathrm{e}(0000-)-2 \mathrm{e}(00--+)+\mathrm{e}(00-+-)+5 \mathrm{e}(00+--)+\mathrm{e}(0-0-+)+ \\
3 \mathrm{e}(0-0+-)+2 \mathrm{e}(0--0+)-5 \mathrm{e}(---++)+\mathrm{e}(--+-+)\end{array}$ \\
\hline 3 & 0 & $w \cdot w \cdot x^{4} \cdot v$ & $\begin{array}{c}4 \mathrm{e}(000-+)+\mathrm{e}(00-0+)-\mathrm{e}(00+0-)-\mathrm{e}(0-+-+)-3 \mathrm{e}(0-++-)- \\
\mathrm{e}(0+--+)-3 \mathrm{e}(0+-+-)-6 \mathrm{e}(0++--)\end{array}$ \\
\hline 3 & 0 & {$\left[x . v, w . x^{4} . v\right]$} & $\begin{array}{l}4 \mathrm{e}(000-+)-2 \mathrm{e}(000+-)+\mathrm{e}(00-0+)-\mathrm{e}(00+0-)-2 \mathrm{e}(0--++)- \\
\mathrm{e}(0-+-+)-\mathrm{e}(0-++-)+\mathrm{e}(0+--+)+3 \mathrm{e}(0+-+-)+6 \mathrm{e}(0++--)\end{array}$ \\
\hline 3 & 0 & {$\left[x . v,\left[x . v, x^{4} . v\right]\right]$} & $\begin{array}{l}2 \mathrm{e}(000-+)-6 \mathrm{e}(000+-)-\mathrm{e}(00-0+)-\mathrm{e}(00+0-)-2 \mathrm{e}(0--++)+ \\
\mathrm{e}(0-+-+)+3 \mathrm{e}(0-++-)-\mathrm{e}(0+--+)-3 \mathrm{e}(0+-+-)-6 \mathrm{e}(0++--)\end{array}$ \\
\hline 3 & 0 & {$\left[x . w, v \cdot x^{4} . v\right]$} & $\begin{aligned} 2 \mathrm{e}(0--++)+\mathrm{e}(0-+-+)+\mathrm{e}(0-++-)-\mathrm{e}(0+--+)-\mathrm{e}(0+-+-)- & \\
2 \mathrm{e}(0++--) & \end{aligned}$ \\
\hline 3 & 0 & {$\left[x \cdot w, w \cdot x^{3} . v\right]$} & $\begin{array}{c}\mathrm{e}(000-+)+\mathrm{e}(000+-)+\mathrm{e}(00-0+)-\mathrm{e}(0-+-+)-\mathrm{e}(0-++-)- \\
\mathrm{e}(0+--+)-\mathrm{e}(0+-+-)-2 \mathrm{e}(0++--)\end{array}$ \\
\hline
\end{tabular}




\begin{tabular}{|c|c|c|c|}
\hline 1 & $\mathrm{~m}$ & $h \in \mathrm{H}$ in terms of $\mathrm{X}$ & $h \in \mathrm{H}$ in terms of the Euler-Lyndon basis \\
\hline 3 & 0 & {$\left[x . w,\left[x . v, x^{2} . w\right]\right]$} & $\begin{array}{c}\mathrm{e}(000-+)+\mathrm{e}(000+-)+\mathrm{e}(00+0-)-2 \mathrm{e}(0--++)-\mathrm{e}(0-+-+)- \\
\mathrm{e}(0-++-)-\mathrm{e}(0+--+)-\mathrm{e}(0+-+-)\end{array}$ \\
\hline 3 & 0 & {$\left[x^{2} . v, v \cdot x^{4} . v\right]$} & $2 \mathrm{e}(000-+)+2 \mathrm{e}(000+-)+\mathrm{e}(00-0+)+\mathrm{e}(00+0-)+\mathrm{e}(0--++)+\mathrm{e}(0++--)$ \\
\hline 3 & 0 & {$\left[x^{2} . v, w \cdot x^{2} . w\right]$} & $\begin{array}{c}4 \mathrm{e}(000-+)+4 \mathrm{e}(000+-)+2 \mathrm{e}(00-0+)+2 \mathrm{e}(00+0-)-\mathrm{e}(0-+-+)- \\
\mathrm{e}(0-++-)-\mathrm{e}(0+--+)-\mathrm{e}(0+-+-)\end{array}$ \\
\hline 3 & 0 & {$\left[x^{2} \cdot v, w \cdot x^{3} \cdot v\right]$} & $\begin{array}{c}3 \mathrm{e}(000-+)+3 \mathrm{e}(000+-)+\mathrm{e}(00-0+)+2 \mathrm{e}(00+0-)+2 \mathrm{e}(0-++-)+ \\
2 \mathrm{e}(0++--)\end{array}$ \\
\hline 3 & 0 & {$\left[x^{2} . v,\left[x . v, x^{2} . w\right]\right]$} & $\begin{array}{c}3 \mathrm{e}(000-+)+3 \mathrm{e}(000+-)+2 \mathrm{e}(00-0+)+\mathrm{e}(00+0-)+2 \mathrm{e}(0--++)+ \\
2 \mathrm{e}(0+--+)\end{array}$ \\
\hline 3 & 1 & {$\left[x . w, w \cdot x^{4} . v\right]$} & $\begin{array}{c}\mathrm{e}(0000+)+\mathrm{e}(00-++)+\mathrm{e}(00+-+)+2 \mathrm{e}(00++-)-\mathrm{e}(0-+0+)+ \\
\mathrm{e}(0+0+-)-\mathrm{e}(--+++)-\mathrm{e}(-+-++)\end{array}$ \\
\hline 3 & 1 & {$\left[x . w,\left[x . v, x^{4} . v\right]\right]$} & $\begin{array}{c}\mathrm{e}(0000+)-3 \mathrm{e}(00-++)-5 \mathrm{e}(00+-+)-6 \mathrm{e}(00++-)-4 \mathrm{e}(0-0++)- \\
\mathrm{e}(0-+0+)-\mathrm{e}(0+0+-)-5 \mathrm{e}(--+++)+\mathrm{e}(-+-++)\end{array}$ \\
\hline 3 & 1 & {$\left[x . w,\left[x . w, x^{2} . w\right]\right]$} & $\begin{array}{c}\mathrm{e}(0000+)-3 \mathrm{e}(00-++)-\mathrm{e}(00+-+)+2 \mathrm{e}(00++-)-2 \mathrm{e}(0-0++)- \\
\mathrm{e}(0-+0+)+\mathrm{e}(0+0+-)+3 \mathrm{e}(--+++)+\mathrm{e}(-+-++)\end{array}$ \\
\hline 3 & 1 & {$\left[x . w,\left[x . w, x^{3} . v\right]\right]$} & $\begin{array}{c}\mathrm{e}(0000+)+3 \mathrm{e}(00-++)+3 \mathrm{e}(00+-+)+4 \mathrm{e}(00++-)+4 \mathrm{e}(0-0++)+ \\
\mathrm{e}(0-+0+)+\mathrm{e}(0+0+-)+5 \mathrm{e}(--+++)-\mathrm{e}(-+-++)\end{array}$ \\
\hline 3 & 1 & {$\left[x^{2} . v, w \cdot x^{4} . v\right]$} & $\begin{array}{c}7 \mathrm{e}(0000+)-7 \mathrm{e}(00-++)-3 \mathrm{e}(00+-+)-6 \mathrm{e}(00++-)-4 \mathrm{e}(0-0++)- \\
\mathrm{e}(0-+0+)+\mathrm{e}(0+0+-)+5 \mathrm{e}(--+++)+5 \mathrm{e}(-+-++)\end{array}$ \\
\hline 3 & 1 & {$\left[x^{2} . v,\left[x . v, x^{4} . v\right]\right]$} & $\begin{array}{c}7 \mathrm{e}(0000+)-3 \mathrm{e}(00-++)+3 \mathrm{e}(00+-+)+2 \mathrm{e}(00++-)-\mathrm{e}(0-+0+)- \\
\mathrm{e}(0+0+-)+\mathrm{e}(--+++)-\mathrm{e}(-+-++)\end{array}$ \\
\hline 3 & 1 & {$\left[x^{2} . v,\left[x . w, x^{2} . w\right]\right]$} & $\begin{array}{c}7 \mathrm{e}(0000+)-3 \mathrm{e}(00-++)-\mathrm{e}(00+-+)+2 \mathrm{e}(00++-)-2 \mathrm{e}(0-0++)- \\
\mathrm{e}(0-+0+)+\mathrm{e}(0+0+-)-15 \mathrm{e}(--+++)-5 \mathrm{e}(-+-++)\end{array}$ \\
\hline 3 & 1 & {$\left[x^{2} . v,\left[x . w, x^{3} . v\right]\right]$} & $\begin{array}{c}7 \mathrm{e}(0000+)+3 \mathrm{e}(00-++)-\mathrm{e}(00+-+)-4 \mathrm{e}(00++-)+\mathrm{e}(0-+0+)+ \\
\mathrm{e}(0+0+-)-\mathrm{e}(--+++)+\mathrm{e}(-+-++)\end{array}$ \\
\hline 3 & 1 & {$\left[x^{2} . w, w \cdot x^{3} \cdot v\right]$} & $\begin{array}{c}5 \mathrm{e}(0000+)-3 \mathrm{e}(00-++)-\mathrm{e}(00+-+)-4 \mathrm{e}(00++-)-2 \mathrm{e}(0-0++)- \\
\mathrm{e}(0-+0+)-\mathrm{e}(0+0+-)+3 \mathrm{e}(--+++)+\mathrm{e}(-+-++)\end{array}$ \\
\hline 3 & 2 & {$\left[x . w,\left[x . w, x^{4} . v\right]\right]$} & $2 \mathrm{e}(0-+++)-\mathrm{e}(0++-+)-\mathrm{e}(0+++-)$ \\
\hline 3 & 2 & {$\left[x^{2} . v,\left[x . w, x^{4} . v\right]\right]$} & $2 \mathrm{e}(0-+++)+\mathrm{e}(0++-+)+\mathrm{e}(0+++-)$ \\
\hline 3 & 2 & {$\left[x^{2} . v,\left[x^{2} . v, x^{4} . v\right]\right]$} & $\begin{array}{c}10 \mathrm{e}(000++)+\mathrm{e}(00+0+)-2 \mathrm{e}(0-+++)+2 \mathrm{e}(0+-++)-\mathrm{e}(0++-+)- \\
3 \mathrm{e}(0+++-)\end{array}$ \\
\hline 3 & 2 & {$\left[x^{2} . w, w \cdot x^{4} \cdot v\right]$} & $3 \mathrm{e}(000++)+\mathrm{e}(00+0+)-\mathrm{e}(0++-+)-2 \mathrm{e}(0+++-)$ \\
\hline 3 & 2 & {$\left[x^{2} . w,\left[x . v, x^{4} . v\right]\right]$} & $3 \mathrm{e}(000++)+\mathrm{e}(0+-++)+\mathrm{e}(0++-+)+2 \mathrm{e}(0+++-)$ \\
\hline 3 & 2 & {$\left[x^{2} . w,\left[x . w, x^{3} . v\right]\right]$} & $\mathrm{e}(000++)+\mathrm{e}(00+0+)-\mathrm{e}(0+-++)-\mathrm{e}(0++-+)-2 \mathrm{e}(0+++-)$ \\
\hline 3 & 3 & {$\left[x^{2} . w,\left[x . w, x^{4} . v\right]\right]$} & $\mathrm{e}(00+++)-2 \mathrm{e}(-++++)$ \\
\hline 3 & 3 & {$\left[x^{2} . w,\left[x^{2} \cdot v, x^{4} \cdot v\right]\right]$} & $2 \mathrm{e}(00+++)-\mathrm{e}(0+0++)-2 \mathrm{e}(-++++)$ \\
\hline 3 & 3 & {$\left[x^{3} . v,\left[x . w, x^{4} . v\right]\right]$} & $\mathrm{e}(00+++)+2 \mathrm{e}(-++++)$ \\
\hline 3 & 4 & {$\left[x^{2} . w,\left[x^{2} \cdot w, x^{4} \cdot v\right]\right]$} & $\mathrm{e}(0++++)$ \\
\hline 4 & -5 & $v \cdot v \cdot v \cdot x^{2} \cdot w$ & $\mathrm{e}(0-----)$ \\
\hline 4 & -4 & $v . v \cdot v \cdot x^{4} . v$ & $4 \mathrm{e}(00----)+\mathrm{e}(0-0---)+2 \mathrm{e}(-----+)$ \\
\hline 4 & -4 & $w \cdot v \cdot v \cdot x^{2} \cdot w$ & $5 \mathrm{e}(00----)+3 \mathrm{e}(0-0---)-5 \mathrm{e}(-----+)$ \\
\hline 4 & -4 & $w \cdot v \cdot v \cdot x^{3} \cdot v$ & $16 \mathrm{e}(00----)+\mathrm{e}(0-0---)+10 \mathrm{e}(-----+)$ \\
\hline 4 & -3 & $w \cdot v \cdot v \cdot x^{4} \cdot v$ & $\begin{array}{l}2 \mathrm{e}(000---)+2 \mathrm{e}(00-0--)+\mathrm{e}(00--0-)-5 \mathrm{e}(0----+)- \\
4 \mathrm{e}(0---+-)-3 \mathrm{e}(0--+--)-2 \mathrm{e}(0-+---)+\mathrm{e}(0+----)\end{array}$ \\
\hline 4 & -3 & $w . w . v \cdot x^{2} \cdot w$ & $\begin{array}{c}15 \mathrm{e}(000---)+5 \mathrm{e}(00-0--)+2 \mathrm{e}(00--0-)-10 \mathrm{e}(0----+)- \\
6 \mathrm{e}(0---++)-3 \mathrm{e}(0--+--)\end{array}$ \\
\hline 4 & -3 & $w \cdot w \cdot v \cdot x^{3} \cdot v$ & $\begin{array}{c}33 \mathrm{e}(000---)+7 \mathrm{e}(00-0--)+4 \mathrm{e}(00--0-)+10 \mathrm{e}(0----+)+ \\
6 \mathrm{e}(0---+-)+3 \mathrm{e}(0--+--)+3 \mathrm{e}(0-+---)\end{array}$ \\
\hline 4 & -3 & $w \cdot w \cdot w \cdot x^{2} \cdot v$ & $7 \mathrm{e}(000---)+2 \mathrm{e}(00-0--)+\mathrm{e}(00--0-)$ \\
\hline 4 & -3 & {$\left[x . v, v . v \cdot x^{4} . v\right]$} & $\begin{array}{c}4 \mathrm{e}(000---)+\mathrm{e}(00-0--)-7 \mathrm{e}(0----+)-6 \mathrm{e}(0---+-)- \\
4 \mathrm{e}(0--+--)-\mathrm{e}(0-+---)+\mathrm{e}(0+----)\end{array}$ \\
\hline 4 & -3 & {$\left[x . v, w . v \cdot x^{2} . w\right]$} & $3 \mathrm{e}(000---)+\mathrm{e}(00-0--)-14 \mathrm{e}(0----+)-8 \mathrm{e}(0---+-)-3 \mathrm{e}(0--+--)$ \\
\hline 4 & -3 & {$\left[x . v, w . v . x^{3} . v\right]$} & $15 \mathrm{e}(000---)+7 \mathrm{e}(00-0--)+4 \mathrm{e}(00--0-)-14 \mathrm{e}(0----+)-$ \\
\hline 4 & -3 & {$\left[x . v, w \cdot w \cdot x^{2} . v\right]$} & $\begin{array}{c}8 \mathrm{e}(0---+-)-5 \mathrm{e}(0--+--)-3 \mathrm{e}(0-+---) \\
3 \mathrm{e}(000---)-\mathrm{e}(00--0-)\end{array}$ \\
\hline 4 & -2 & $w \cdot w \cdot v \cdot x^{4} \cdot v$ & $6 \mathrm{e}(0000--)+\mathrm{e}(00---+)-\mathrm{e}(00--+-)-\mathrm{e}(0-0--+)-\mathrm{e}(0-0-+-)$ \\
\hline 4 & -2 & $w \cdot w \cdot w \cdot x^{3} \cdot v$ & $4 \mathrm{e}(0000--)+\mathrm{e}(000-0-)$ \\
\hline 4 & -2 & {$\left[x . v, w . v \cdot x^{4} . v\right]$} & $5 \mathrm{e}(0000--)-11 \mathrm{e}(00---+)-8 \mathrm{e}(00--+-)-3 \mathrm{e}(00-+--)-$ \\
\hline 4 & -2 & {$\left[x . v, w . w . x^{2} . w\right]$} & $\begin{array}{c}4 \mathrm{e}(0-0--+)-3 \mathrm{e}(0-0-+-)-\mathrm{e}(0-0+--)-\mathrm{e}(0--0-+)-\mathrm{e}(0--0+-) \\
\mathrm{e}(0000--)+6 \mathrm{e}(00---+)+3 \mathrm{e}(00--+-)+\mathrm{e}(00-+--)+\end{array}$ \\
\hline 4 & -2 & {$\left[x \cdot v, w \cdot w \cdot x^{3} \cdot v\right]$} & $\begin{array}{c}3 \mathrm{e}(0-0--+)+2 \mathrm{e}(0-0-+-)+\mathrm{e}(0-0+--)+\mathrm{e}(0--0-+)+\mathrm{e}(0--0+-) \\
25 \mathrm{e}(0000--)+9 \mathrm{e}(000-0-)+18 \mathrm{e}(00---+)+9 \mathrm{e}(00--+-)+\end{array}$ \\
\hline & & & $\begin{array}{c}3 \mathrm{e}(00-+--)+9 \mathrm{e}(0-0--+)+4 \mathrm{e}(0-0-+-)+\mathrm{e}(0-0+--)+ \\
3 \mathrm{e}(0--0-+)+\mathrm{e}(0--0+-)\end{array}$ \\
\hline 4 & -2 & {$\left[x . v,\left[x . v, v \cdot x^{4} . v\right]\right]$} & $\begin{array}{c}\mathrm{e}(000-0-)+10 \mathrm{e}(00---+)+4 \mathrm{e}(00--+-)+5 \mathrm{e}(0-0--+)+ \\
2 \mathrm{e}(0-0-+-)+3 \mathrm{e}(0--0-+)+\mathrm{e}(0-0-0+-)+2 \mathrm{e}(0---0+)\end{array}$ \\
\hline
\end{tabular}


Appendix D. A Pseudo-Hall basis of $\mathfrak{g}$ for $d=3$

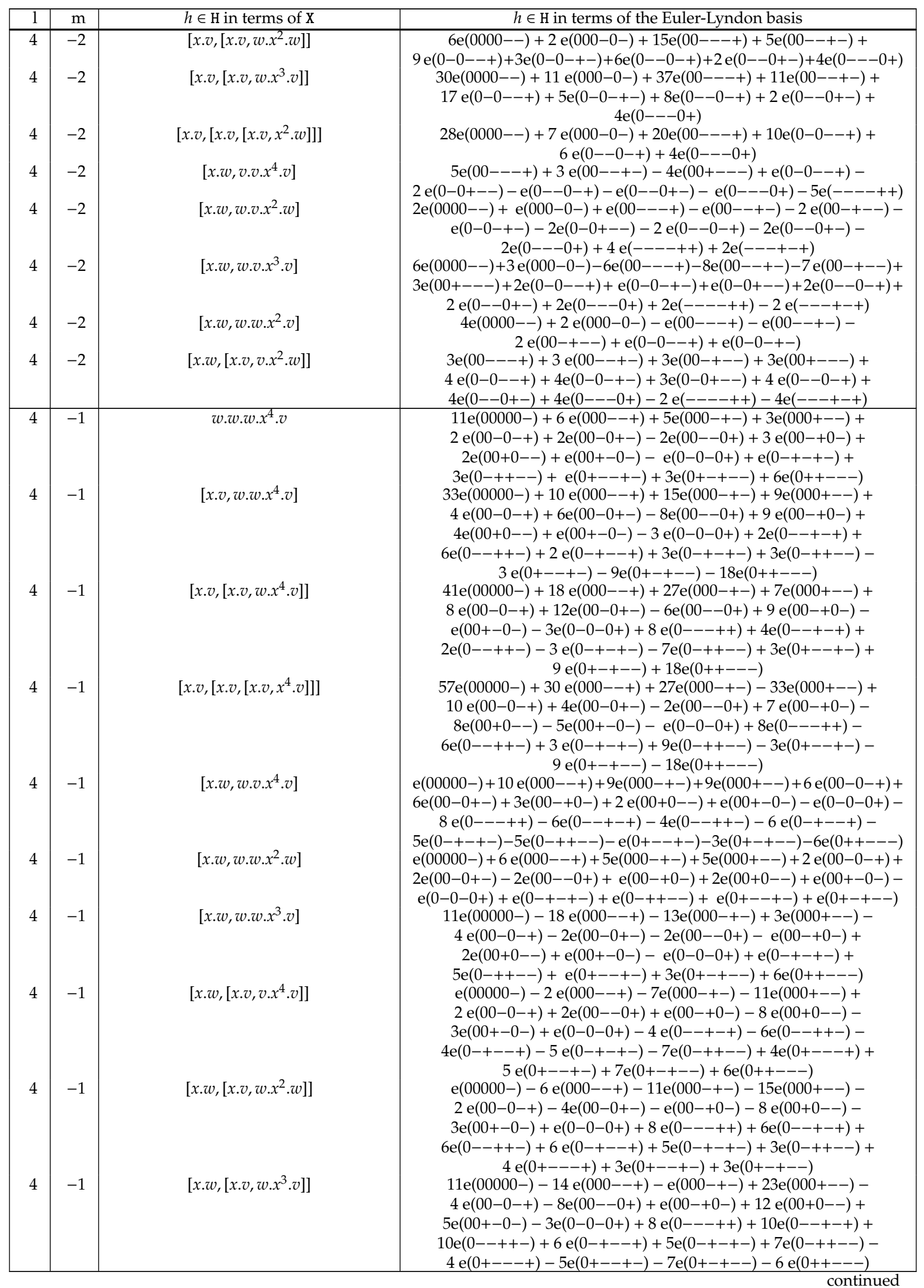




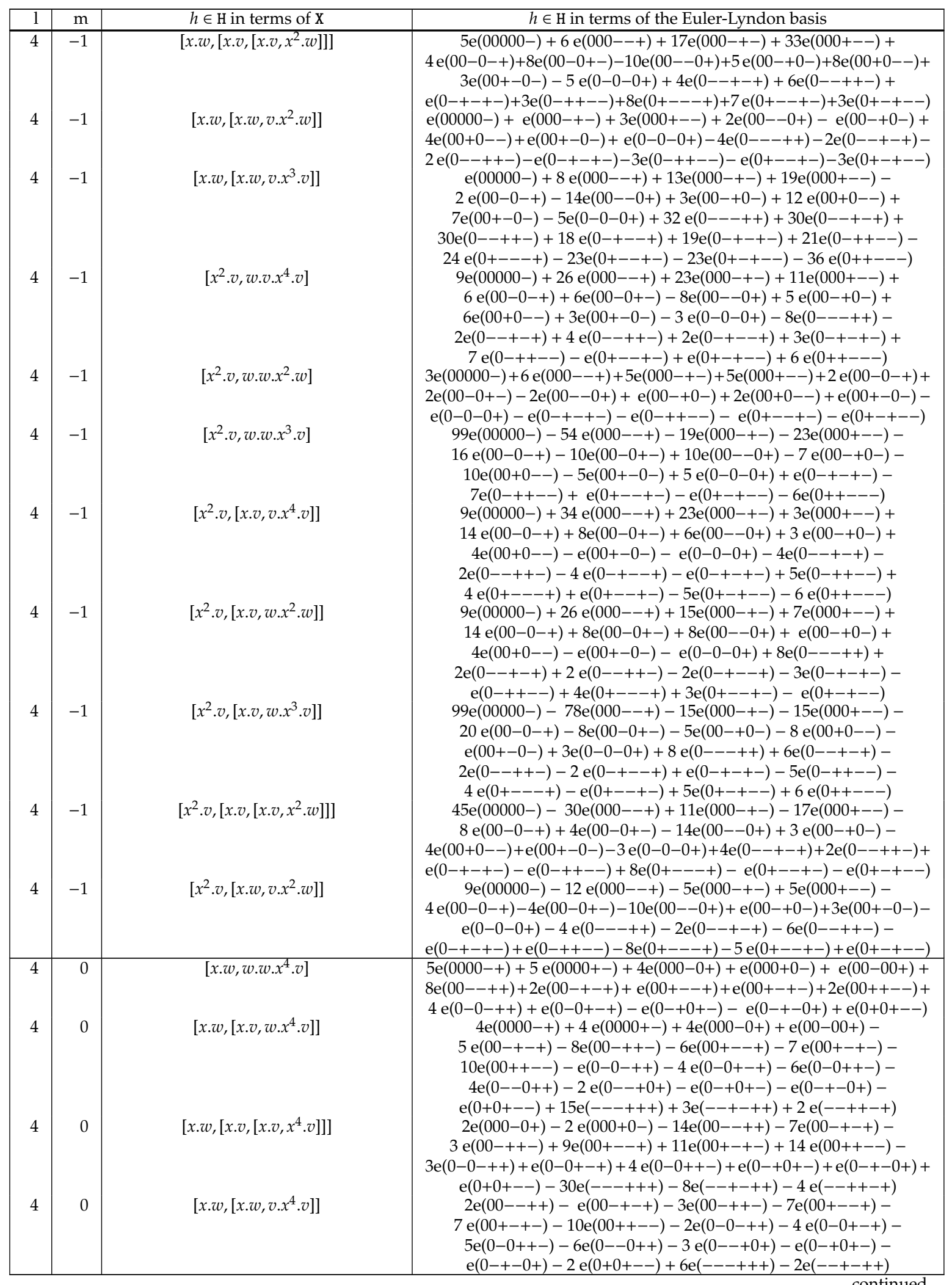


Appendix D. A Pseudo-Hall basis of $\mathfrak{g}$ for $d=3$

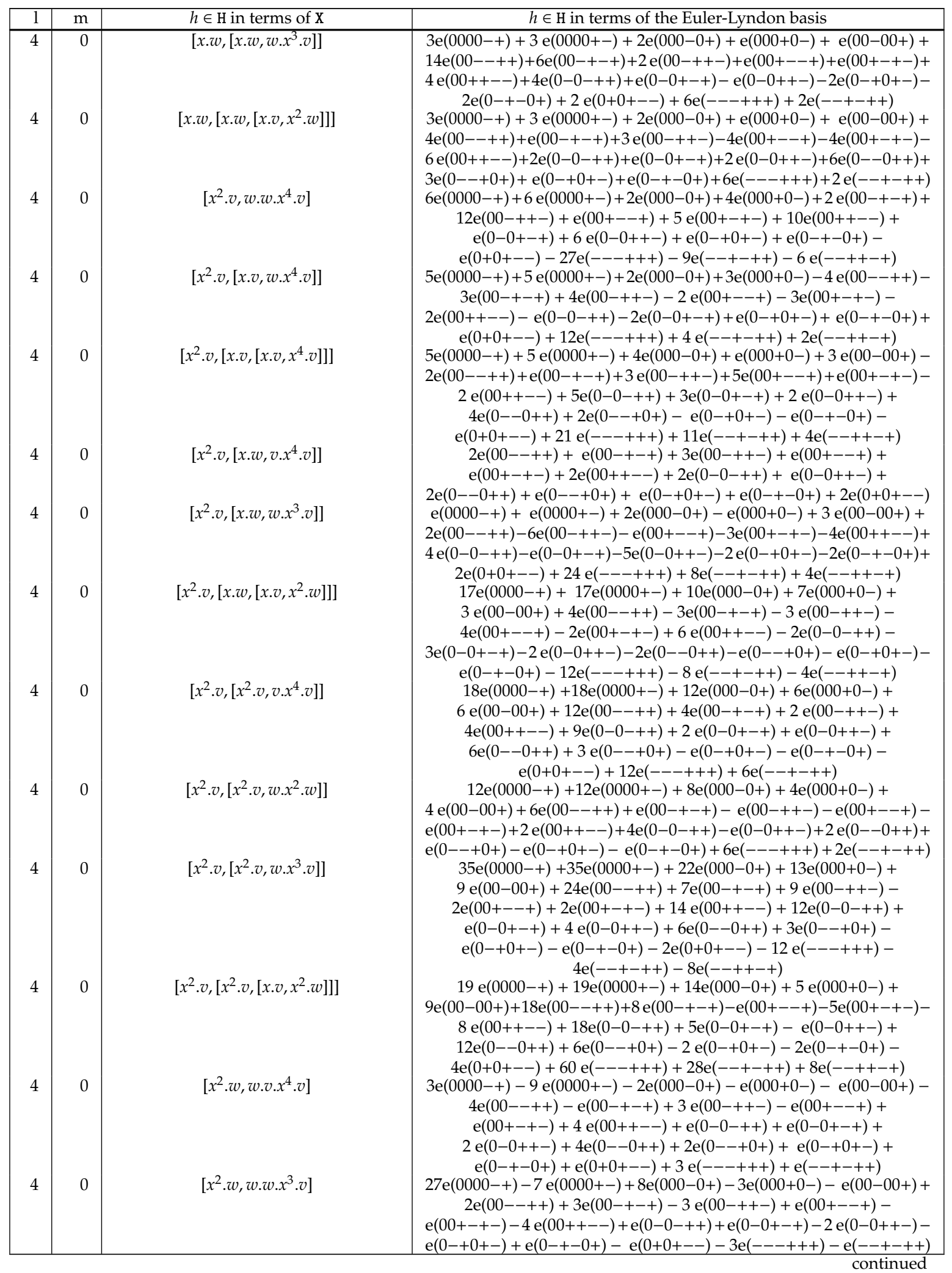




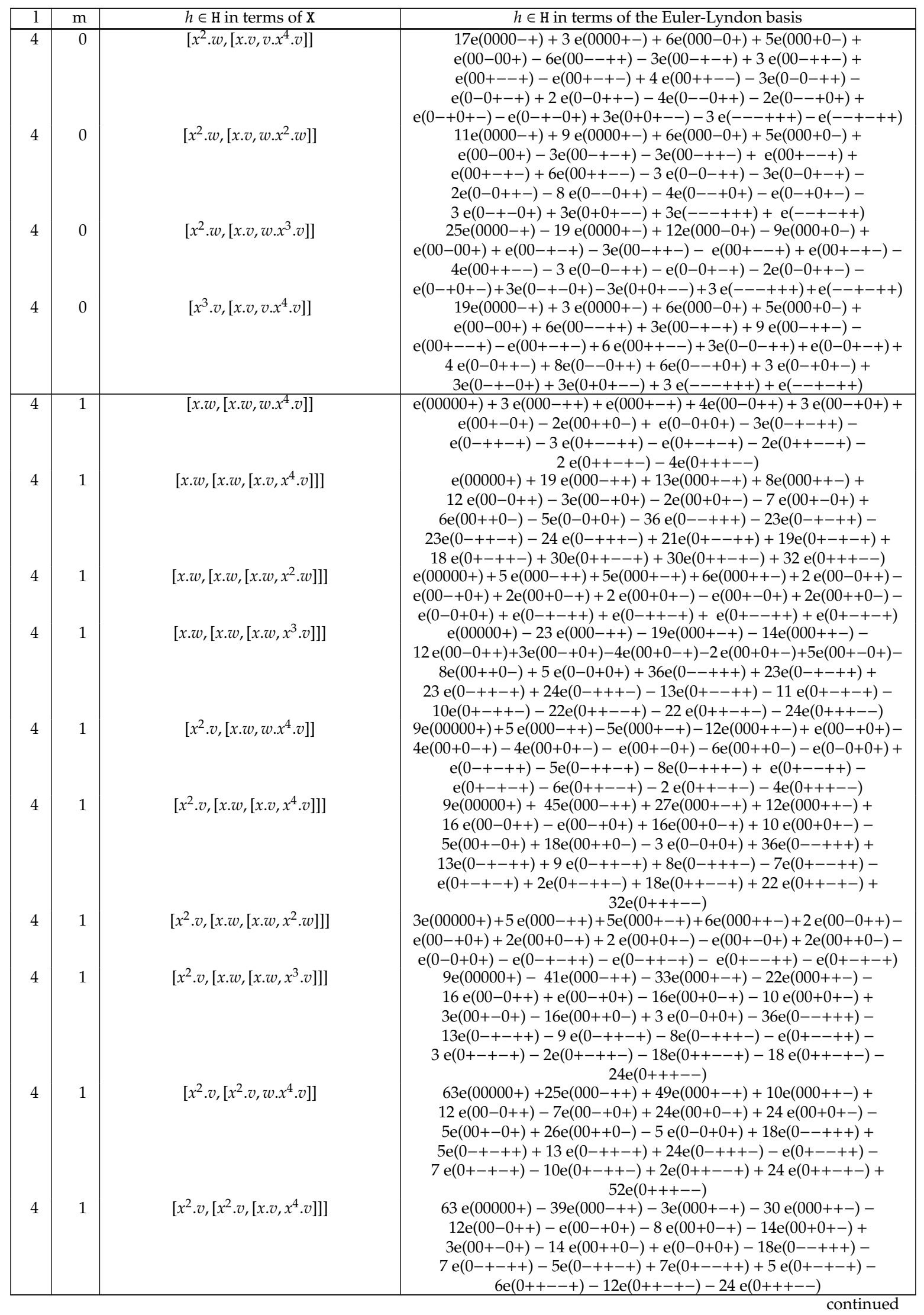


Appendix D. A Pseudo-Hall basis of $\mathfrak{g}$ for $d=3$

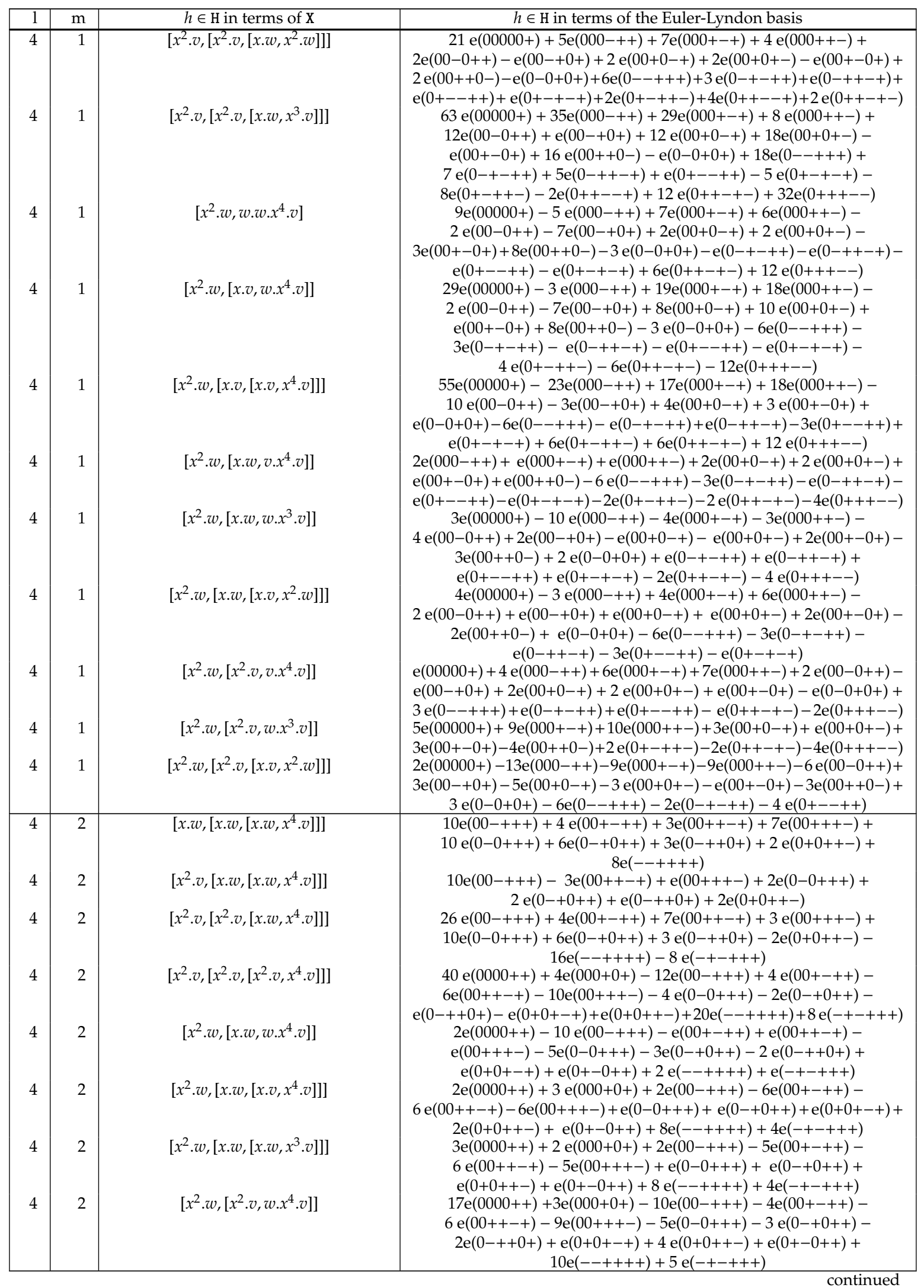




\begin{tabular}{|c|c|c|c|}
\hline 1 & $\mathrm{~m}$ & $h \in \mathrm{H}$ in terms of X & $h \in \mathrm{H}$ in terms of the Euler-Lyndon basis \\
\hline 4 & 2 & {$\left[x^{2} . w,\left[x^{2} . v,\left[x . v, x^{4} . v\right]\right]\right]$} & $\begin{array}{c}37 \mathrm{e}(0000++)+6 \mathrm{e}(000+0+)-2 \mathrm{e}(00-+++)+3 \mathrm{e}(00+-++)- \\
\mathrm{e}(00++-+)+4 \mathrm{e}(00+++-)-\mathrm{e}(0-0+++)-\mathrm{e}(0-+0++)-3 \mathrm{e}(0+0+-+)- \\
2 \mathrm{e}(0+0++-)-\mathrm{e}(0+-0++)-4 \mathrm{e}(--++++)-2 \mathrm{e}(-+-+++) \\
5 \mathrm{e}(0000++)+\mathrm{e}(000+0+)-\mathrm{e}(00+-++)-\mathrm{e}(00++-+)\end{array}$ \\
\hline 4 & 2 & {$\left[x^{2} . w,\left[x^{2} . v,\left[x . w, x^{3} . v\right]\right]\right]$} & $\begin{array}{c}4 \mathrm{e}(0000++)+\mathrm{e}(000+0+)+2 \mathrm{e}(00-+++)-2 \mathrm{e}(00+-++)-\mathrm{e}(00++-+)- \\
5 \mathrm{e}(00+++-)+\mathrm{e}(0-0+++)+\mathrm{e}(0-+0++)+2 \mathrm{e}(0+0+-+)+ \\
3 \mathrm{e}(0+0++-)+\mathrm{e}(0+-0++)+4 \mathrm{e}(--++++)+2 \mathrm{e}(-+-+++)\end{array}$ \\
\hline 4 & 2 & {$\left[x^{2} . w,\left[x^{2} . w, w \cdot x^{3} . v\right]\right]$} & $\begin{array}{c}22 \mathrm{e}(0000++)+5 \mathrm{e}(000+0+)-3 \mathrm{e}(00++-+)-\mathrm{e}(00+++-)- \\
\mathrm{e}(0+0+-+)+\mathrm{e}(0+0++-)\end{array}$ \\
\hline 4 & 2 & {$\left[x^{3} . v,\left[x . w, w . x^{4} . v\right]\right]$} & $3 \mathrm{e}(0000++)-4 \mathrm{e}(00-+++)-\mathrm{e}(00+++-)-2 \mathrm{e}(0-0+++)-$ \\
\hline 4 & 2 & {$\left[x^{3} . v,\left[x . w,\left[x . v, x^{4} . v\right]\right]\right]$} & $\begin{array}{c}\mathrm{e}(0000++)+3 \mathrm{e}(000+0+)+8 \mathrm{e}(00-+++)-\mathrm{e}(00+-++)+\mathrm{e}(00++-+)+ \\
6 \mathrm{e}(00+++-)+4 \mathrm{e}(0-0+++)+2 \mathrm{e}(0+0++-)-8 \mathrm{e}(--++++)- \\
4 \mathrm{e}(-+-+++)\end{array}$ \\
\hline 4 & 3 & {$\left[x^{2} . w,\left[x . w,\left[x . w, x^{4} . v\right]\right]\right]$} & $2 \mathrm{e}(000+++)+2 \mathrm{e}(00+0++)+\mathrm{e}(00++0+)+4 \mathrm{e}(0-++++)-$ \\
\hline 4 & 3 & {$\left[x^{2} . w,\left[x^{2} . v,\left[x . w, x^{4} . v\right]\right]\right]$} & $\begin{array}{l}2 \mathrm{e}(0+-+++)-3 \mathrm{e}(0++-++)-4 \mathrm{e}(0+++-+)-5 \mathrm{e}(0++++-) \\
10 \mathrm{e}(000+++)+2 \mathrm{e}(00+0++)+\mathrm{e}(00++0+)-4 \mathrm{e}(0-++++)+ \\
2 \mathrm{e}(0+-+++)-3 \mathrm{e}(0++-++)-4 \mathrm{e}(0+++-+)-5 \mathrm{e}(0++++-)\end{array}$ \\
\hline 4 & 3 & {$\left[x^{2} . w,\left[x^{2} . v,\left[x^{2} . v, x^{4} . v\right]\right]\right]$} & $\begin{array}{c}97 \mathrm{e}(000+++)+22 \mathrm{e}(00+0++)+10 \mathrm{e}(00++0+)-4 \mathrm{e}(0-++++)+ \\
8 \mathrm{e}(0+-+++)-3 \mathrm{e}(0++-++)-8 \mathrm{e}(0+++-+)-15 \mathrm{e}(0++++-)\end{array}$ \\
\hline 4 & 3 & {$\left[x^{2} \cdot w,\left[x^{2} \cdot w, w \cdot x^{4} \cdot v\right]\right]$} & $\begin{array}{c}15 \mathrm{e}(000+++)+5 \mathrm{e}(00+0++)+2 \mathrm{e}(00++0+)-3 \mathrm{e}(0++-++)- \\
6 \mathrm{e}(0+++-+)-10 \mathrm{e}(0++++-)\end{array}$ \\
\hline 4 & 3 & {$\left[x^{2} . w,\left[x^{2} . w,\left[x . v, x^{4} . v\right]\right]\right]$} & $\begin{array}{c}33 \mathrm{e}(000+++)+7 \mathrm{e}(00+0++)+4 \mathrm{e}(00++0+)+3 \mathrm{e}(0+-+++)+ \\
3 \mathrm{e}(0++-++)+6 \mathrm{e}(0+++-+)+10 \mathrm{e}(0++++-)\end{array}$ \\
\hline 4 & 3 & {$\left[x^{2} . w,\left[x^{2} . w,\left[x . w, x^{3} . v\right]\right]\right]$} & $12 \mathrm{e}(000+++)+\mathrm{e}(00+0++)+\mathrm{e}(00++0+)+3 \mathrm{e}(0+-+++)+$ \\
\hline 4 & 3 & {$\left[x^{3} . v,\left[x . w,\left[x . w, x^{4} . v\right]\right]\right]$} & $2 \mathrm{e}(000+++)+2 \mathrm{e}(00+0++)+\mathrm{e}(00++0+)-4 \mathrm{e}(0-++++)-$ \\
\hline 4 & 3 & {$\left[x^{3} . v,\left[x^{2} . v,\left[x^{2} . v, x^{4} . v\right]\right]\right]$} & $\begin{array}{c}2 \mathrm{e}(0+-+++)+3 \mathrm{e}(0++-++)+6 \mathrm{e}(0+++-+)+7 \mathrm{e}(0++++-) \\
37 \mathrm{e}(000+++)+16 \mathrm{e}(00+0++)+8 \mathrm{e}(00++0+)+4 \mathrm{e}(0-++++)- \\
4 \mathrm{e}(0+-+++)-\mathrm{e}(0++-++)+10 \mathrm{e}(0+++-+)+21 \mathrm{e}(0++++-)\end{array}$ \\
\hline 4 & 4 & {$\left[x^{2} . w,\left[x^{2} . w,\left[x . w, x^{4} . v\right]\right]\right]$} & $6 \mathrm{e}(00++++)-\mathrm{e}(0+0+++)-10 \mathrm{e}(-+++++)$ \\
\hline 4 & 4 & {$\left[x^{2} . w,\left[x^{2} . w,\left[x^{2} . v, x^{4} . v\right]\right]\right]$} & $12 \mathrm{e}(00++++)-7 \mathrm{e}(0+0+++)-10 \mathrm{e}(-+++++)$ \\
\hline 4 & 4 & {$\left[x^{3} . v,\left[x^{2} . w,\left[x . w, x^{4} . v\right]\right]\right]$} & $6 \mathrm{e}(00++++)+\mathrm{e}(0+0+++)-10 \mathrm{e}(-+++++)$ \\
\hline 4 & 5 & {$\left[x^{2} . w,\left[x^{2} . w,\left[x^{2} . w, x^{4} . v\right]\right]\right]$} & $\mathrm{e}(0+++++)$ \\
\hline
\end{tabular}


Appendix D. A Pseudo-Hall basis of $\mathfrak{g}$ for $d=3$

\section{Tuple B of relations}

\begin{tabular}{|c|c|c|c|}
\hline 1 & $\mathrm{~m}$ & $\mathrm{LM}(b)$ for $b \in \mathrm{B}$ & $b-\operatorname{LM}(b)$ \\
\hline 1 & 2 & $x^{3} \cdot w$ & 0 \\
\hline 1 & 3 & $x^{5} . v$ & 0 \\
\hline$\frac{2}{2}$ & -3 & {$\left[v, x^{1} . v\right]$} & $-4[v, w]$ \\
\hline 2 & -2 & $4\left[w, x^{1} \cdot v\right]$ & $-4\left[v, x^{1} . w\right]+\left[v, x^{2} . v\right]$ \\
\hline$\frac{2}{2}$ & -1 & $6\left[x^{1} . v, x^{1} . w\right]$ & $-\left[v, x^{3} . v\right]+4\left[w, x^{1} . w\right]-2\left[w, x^{2} . v\right]$ \\
\hline 2 & -1 & $3\left[x^{1} . v, x^{2} . v\right]$ & $-12\left[v, x^{2} . w\right]-\left[v, x^{3} . v\right]+16\left[w, x^{1} . w\right]+4\left[w, x^{2} . v\right]$ \\
\hline$\frac{5}{2}$ & 0 & {$\left[x^{1} . v, x^{3} . v\right]$} & $-\left[v, x^{4} . v\right]+12\left[w, x^{2} . w\right]+2\left[w, x^{3} . v\right]-6\left[x^{1} . v, x^{2} . w\right]$ \\
\hline 2 & 0 & $4\left[x^{1} \cdot w, x^{2} \cdot v\right]$ & {$\left[v, x^{4} . v\right]-8\left[w, x^{2} . w\right]$} \\
\hline 2 & 1 & $4\left[x^{2} . v, x^{2} . w\right]$ & $-\left[x^{1} . v, x^{4} . v\right]+8\left[x^{1} . w, x^{2} . w\right]-4\left[x^{1} . w, x^{3} . v\right]$ \\
\hline 2 & 1 & $2\left[x^{2} . v, x^{3} . v\right]$ & $4\left[w, x^{4} . v\right]-3\left[x^{1} . v, x^{4} . v\right]+48\left[x^{1} . w, x^{2} . w\right]-8\left[x^{1} . w, x^{3} . v\right]$ \\
\hline 2 & 2 & $8\left[x^{2} . w, x^{3} . v\right]$ & $4\left[x^{1} . w, x^{4} . v\right]+\left[x^{2} . v, x^{4} . v\right]$ \\
\hline 2 & 3 & {$\left[x^{3} . v, x^{4} . v\right]$} & $12\left[x^{2} . w, x^{4} . v\right]$ \\
\hline 3 & -5 & {$[v,[v, w]]$} & 0 \\
\hline 3 & -4 & {$\left[v,\left[v, x^{2} . v\right]\right]$} & $-7\left[v,\left[v, x^{1} . w\right]\right]$ \\
\hline 3 & -4 & $8[w,[v, w]]$ & $5\left[v,\left[v, x^{1} . w\right]\right]$ \\
\hline 3 & -4 & $4\left[x^{1} \cdot v,[v, w]\right]$ & $7\left[v,\left[v, x^{1} . w\right]\right]$ \\
\hline 3 & -3 & $6\left[w,\left[v, x^{2} . v\right]\right]$ & $48\left[v,\left[v, x^{2} . w\right]\right]+7\left[v,\left[v, x^{3} . v\right]\right]+240\left[w,\left[v, x^{1} . w\right]\right]$ \\
\hline 3 & -3 & $3\left[x^{1} . v,\left[v, x^{1} . w\right]\right]$ & $-6\left[v,\left[v, x^{2} . w\right]\right]-\left[v,\left[v, x^{3} . v\right]\right]-42\left[w,\left[v, x^{1} . w\right]\right]$ \\
\hline 3 & -3 & $3\left[x^{1} . v,\left[v, x^{2} . v\right]\right]$ & {$\left[v,\left[v, x^{3} . v\right]\right]$} \\
\hline 3 & -3 & $12\left[x^{1} . w,[v, w]\right]$ & $-9\left[v,\left[v, x^{2} . w\right]\right]-\left[v,\left[v, x^{3} . v\right]\right]-60\left[w,\left[v, x^{1} . w\right]\right]$ \\
\hline 3 & -3 & $2\left[x^{2} \cdot v,[v, w]\right]$ & $7\left[v,\left[v, x^{2} . w\right]\right]+\left[v,\left[v, x^{3} . v\right]\right]+28\left[w,\left[v, x^{1} . w\right]\right]$ \\
\hline 3 & -2 & {$\left[w,\left[w, x^{1} . w\right]\right]$} & 0 \\
\hline 3 & -2 & $3\left[x^{1} \cdot v,\left[v, x^{3} \cdot v\right]\right]$ & $48\left[w,\left[v, x^{2} \cdot w\right]\right]+4\left[w,\left[v, x^{3} . v\right]\right]+8\left[w,\left[w, x^{2} . v\right]\right]+12\left[x^{1} \cdot v,\left[v, x^{2} . w\right]\right]$ \\
\hline 3 & -2 & $3\left[x^{1} . v,\left[w, x^{2} . v\right]\right]$ & $\begin{array}{l}-6\left[w,\left[v, x^{2} . w\right]\right]+\left[w,\left[v, x^{3} . v\right]\right]+2\left[w,\left[w, x^{2} . v\right]\right]+3\left[x^{1} . v,\left[v, x^{2} . w\right]\right]- \\
6\left[x^{1} . v,\left[w, x^{1} \cdot w\right]\right]\end{array}$ \\
\hline 3 & -2 & $12\left[x^{1} . w,\left[v, x^{1} \cdot w\right]\right]$ & $\begin{array}{l}{\left[v,\left[v, x^{4} . v\right]\right]-18\left[w,\left[v, x^{2} . w\right]\right]+2\left[w,\left[v, x^{3} . v\right]\right]+3\left[x^{1} . v,\left[v, x^{2} . w\right]\right]-} \\
18\left[x^{1} . v,\left[w, x^{1} . w\right]\right]\end{array}$ \\
\hline 3 & -2 & $3\left[x^{1} . w,\left[v, x^{2} . v\right]\right]$ & $\begin{array}{l}{\left[v,\left[v, x^{4} . v\right]\right]-18\left[w,\left[v, x^{2} . w\right]\right]-4\left[w,\left[w, x^{2} . v\right]\right]+3\left[x^{1} . v,\left[v, x^{2} . w\right]\right]-} \\
6\left[x^{1} . v,\left[w, x^{1} . w\right]\right]\end{array}$ \\
\hline 3 & -2 & $18\left[x^{2} . v,\left[v, x^{1} \cdot w\right]\right]$ & $\begin{array}{l}3\left[v,\left[v, x^{4} . v\right]\right]-138\left[w,\left[v, x^{2} . w\right]\right]+2\left[w,\left[v, x^{3} . v\right]\right]-32\left[w,\left[w, x^{2} . v\right]\right]+ \\
15\left[x^{1} . v,\left[v, x^{2} . w\right]\right]-90\left[x^{1} . v,\left[w, x^{1} . w\right]\right]\end{array}$ \\
\hline 3 & -2 & $9\left[x^{2} . v,\left[v, x^{2} . v\right]\right]$ & $\begin{array}{l}6\left[v,\left[v, x^{4} . v\right]\right]-348\left[w,\left[v, x^{2} . w\right]\right]-20\left[w,\left[v, x^{3} . v\right]\right]-40\left[w,\left[w, x^{2} . v\right]\right]- \\
6\left[x^{1} . v,\left[v, x^{2} . w\right]\right]-108\left[x^{1} . v,\left[w, x^{1} . w\right]\right]\end{array}$ \\
\hline 3 & -2 & $72\left[x^{2} . w,[v, w]\right]$ & $\begin{array}{l}3\left[v,\left[v, x^{4} \cdot v\right]\right]-132\left[w,\left[v, x^{2} . w\right]\right]+4\left[w,\left[v, x^{3} . v\right]\right]-16\left[w,\left[w, x^{2} . v\right]\right]- \\
6\left[x^{1}, v,\left[v, x^{2} \cdot w\right]\right]-108\left[x^{1}, v,\left[w, x^{1} . w\right]\right]\end{array}$ \\
\hline 3 & -2 & $4\left[x^{3} . v,[v, w]\right]$ & {$\left[v,\left[v, x^{4} . v\right]\right]-12\left[w,\left[v, x^{2} . w\right]\right]+6\left[x^{1} . v,\left[v, x^{2} . w\right]\right]-12\left[x^{1} . v,\left[w, x^{1} . w\right]\right]$} \\
\hline 3 & -1 & {$\left[x^{1} . w,\left[w, x^{1} . w\right]\right]$} & {$\left[w,\left[w, x^{2} . w\right]\right]$} \\
\hline 3 & -1 & $44\left[x^{1} . w,\left[w, x^{2} . v\right]\right]$ & $\begin{array}{l}5\left[w,\left[v, x^{4} . v\right]\right]-8\left[w,\left[w, x^{2} . w\right]\right]+12\left[w,\left[w, x^{3} . v\right]\right]-2\left[x^{1} . v,\left[v, x^{4} . v\right]\right]+ \\
72\left[x^{1} . v,\left[w, x^{2} . w\right]\right]+4\left[x^{1} . v,\left[w, x^{3} . v\right]\right]+6\left[x^{1} . v,\left[x^{1} . v, x^{2} . w\right]\right]+\end{array}$ \\
\hline 3 & -1 & $33\left[x^{2} . v,\left[v, x^{2} . w\right]\right]$ & $\begin{array}{l}17\left[w,\left[v, x^{4} . v\right]\right]+492\left[w,\left[w, x^{2} . w\right]\right]+54\left[w,\left[w, x^{3} . v\right]\right]+13\left[x^{1} . v,\left[v, x^{4} . v\right]\right]- \\
72\left[x^{1} . v,\left[w, x^{2} . w\right]\right]+40\left[x^{1} . v,\left[w, x^{3} . v\right]\right]+93\left[x^{1} . v,\left[x^{1} . v, x^{2} . w\right]\right]+ \\
414\left[x^{1} . w,\left[v, x^{2} . w\right]\right]+31\left[x^{1} . w,\left[v, x^{3} . v\right]\right]\end{array}$ \\
\hline 3 & -1 & $11\left[x^{2} . v,\left[v, x^{3} . v\right]\right]$ & $\begin{array}{l}-8\left[w,\left[v, x^{4} . v\right]\right]+48\left[w,\left[w, x^{2} . w\right]\right]+16\left[w,\left[w, x^{3} . v\right]\right]+\left[x^{1} \cdot v,\left[v, x^{4} . v\right]\right]- \\
36\left[x^{1} . v,\left[w, x^{2} . w\right]\right]-2\left[x^{1} . v,\left[w, x^{3} . v\right]\right]+30\left[x^{1} . v,\left[x^{1} . v, x^{2} . w\right]\right]- \\
24\left[x^{1} . w,\left[v, x^{2} . w\right]\right]-12\left[x^{1} . w,\left[v, x^{3} . v\right]\right]\end{array}$ \\
\hline 3 & -1 & $22\left[x^{2} . v,\left[w, x^{1} . w\right]\right]$ & 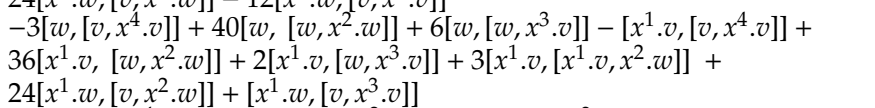 \\
\hline 3 & -1 & $44\left[x^{2} . v,\left[w, x^{2} . v\right]\right]$ & 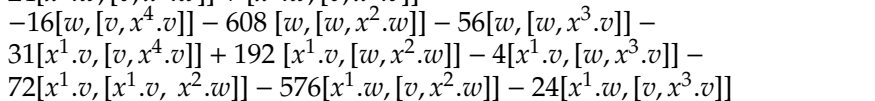 \\
\hline 3 & -1 & $528\left[x^{2} . w,\left[v, x^{1} . w\right]\right]$ & $\begin{array}{l}56\left[w,\left[v, x^{4} . v\right]\right]+3360\left[w,\left[w, x^{2} . w\right]\right]+416\left[w,\left[w, x^{3} . v\right]\right]+ \\
37\left[x^{1} . v,\left[v, x^{4} \cdot v\right]\right]-408\left[x^{1} \cdot v,\left[w, x^{2} . v\right]\right]+300\left[x^{1} . v,\left[w, x^{3} . v\right]\right]+\end{array}$ \\
\hline 3 & -1 & $132\left[x^{2} . w,\left[v, x^{2} . v\right]\right]$ & $\begin{array}{l}516\left[x^{1} \cdot v,\left[x^{1} \cdot v, x^{2} \cdot w\right]\right]+2016\left[x^{1} \cdot w,\left[v, x^{2} \cdot w\right]\right]+128\left[x^{1} \cdot w,\left[v, x^{5} \cdot v\right]\right] \\
64\left[w,\left[v, x^{4} \cdot v\right]\right]+1728\left[w,\left[w, x^{2} \cdot w\right]\right]+224\left[w,\left[w, x^{3} \cdot v\right]\right]+ \\
47\left[x^{1} \cdot v,\left[v, x^{4} \cdot v\right]\right]+24\left[x^{1} \cdot v,\left[w, x^{2} \cdot w\right]\right]+148\left[x^{1} \cdot v,\left[w, x^{3} \cdot v\right]\right]+\end{array}$ \\
\hline 3 & -1 & $88\left[x^{3} \cdot v,\left[v, x^{1} \cdot w\right]\right]$ & 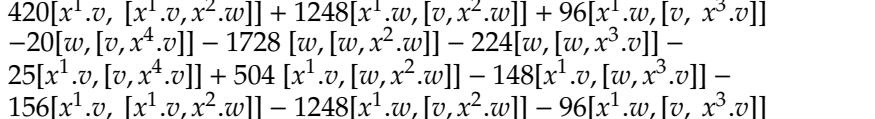 \\
\hline
\end{tabular}




\begin{tabular}{|c|c|c|c|}
\hline 1 & $\mathrm{~m}$ & $\mathrm{LM}(b)$ for $b \in \mathrm{B}$ & $b-\operatorname{LM}(b)$ \\
\hline 3 & -1 & $22\left[x^{3} \cdot v,\left[v, x^{2} . v\right]\right]$ & $\begin{array}{l}-100\left[w,\left[v, x^{4} . v\right]\right]-2304\left[w,\left[w, x^{2} . w\right]\right]-240\left[w,\left[w, x^{3} . v\right]\right]- \\
15\left[x^{1} . v,\left[v, x^{4} . v\right]\right]+408\left[x^{1} . v,\left[w, x^{2} . w\right]\right]-212\left[x^{1} . v,\left[w, x^{3} . v\right]\right]- \\
252\left[x^{1} . v,\left[x^{1} . v, x^{2} . w\right]\right]-2016\left[x^{1} . w,\left[v, x^{2} . w\right]\right]-128\left[x^{1} . w,\left[v, x^{3} . v\right]\right] \\
9\left[w,\left[v, x^{4} . v\right]\right]+672\left[w,\left[w, x^{2} . w\right]\right]+92\left[w,\left[w, x^{3} . v\right]\right]+3\left[x^{1} . v,\left[v, x^{4} . v\right]\right]- \\
108\left[x^{1} . v,\left[w, x^{2} . w\right]\right]+60\left[x^{1} . v,\left[w, x^{3} . v\right]\right]+90\left[x^{1} . v,\left[x^{1} . v, x^{2} . w\right]\right]+ \\
456\left[x^{1} . w,\left[v, x^{2} . w\right]\right]+8\left[x^{1} . w,\left[v, x^{3} . v\right]\right]\end{array}$ \\
\hline 3 & 0 & {$\left[x^{1} . w,\left[w, x^{2} . w\right]\right]$} & 0 \\
\hline 3 & 0 & $48\left[x^{2} . w,\left[v, x^{2} . w\right]\right]$ & $\begin{array}{l}-8\left[w,\left[w, x^{4} . v\right]\right]+2\left[x^{1} . v,\left[x^{1} . v, x^{4} . v\right]\right]+3\left[x^{2} \cdot v,\left[v, x^{4} . v\right]\right]- \\
24\left[x^{2} . v,\left[w, x^{2} . w\right]\right]-12\left[x^{2} . v,\left[w, x^{3} . v\right]\right]-12\left[x^{2} . v,\left[x^{1} . v, x^{2} . w\right]\right]\end{array}$ \\
\hline 3 & 0 & $8\left[x^{2} . w,\left[v, x^{3} . v\right]\right]$ & $\begin{array}{l}4\left[x^{1} \cdot v,\left[w, x^{4} \cdot v\right]\right]+3\left[x^{1} \cdot v,\left[x^{1} \cdot v, x^{4} \cdot v\right]\right]+12\left[x^{1} \cdot w,\left[v, x^{4} \cdot v\right]\right]- \\
16\left[x^{1} \cdot w,\left[w, x^{3} \cdot v\right]\right]+48\left[x^{1} \cdot w,\left[x^{1} \cdot v, x^{2} \cdot w\right]\right]+4\left[x^{2} \cdot v,\left[v, x^{4} \cdot v\right]\right]- \\
12\left[x^{2} \cdot v,\left[w, x^{2} \cdot w\right]\right]-2\left[x^{2} \cdot v,\left[w, x^{3} \cdot v\right]\right]+30\left[x^{2} \cdot v,\left[x^{1} \cdot v, x^{2} \cdot w\right]\right]\end{array}$ \\
\hline 3 & 0 & $32\left[x^{2} . w,\left[w, x^{1} \cdot w\right]\right]$ & $\begin{array}{l}8\left[x^{1} \cdot w,\left[w, x^{3} \cdot v\right]\right]+24\left[x^{1} \cdot w,\left[x^{1} \cdot v, x^{2} \cdot w\right]\right]-\left[x^{2} \cdot v,\left[v, x^{4} \cdot v\right]\right]+ \\
40\left[x^{2} \cdot v,\left[w, x^{2} \cdot w\right]\right]\end{array}$ \\
\hline 3 & 0 & $16\left[x^{2} . w,\left[w, x^{2} . v\right]\right]$ & 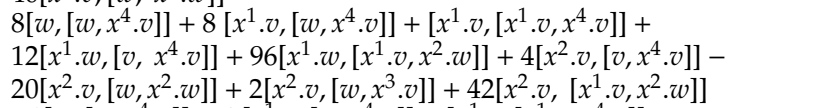 \\
\hline 3 & 0 & $8\left[x^{3} . v,\left[v, x^{2} . w\right]\right]$ & $\begin{array}{l}-8\left[w,\left[w, x^{4} . v\right]\right]-4\left[x^{1} . v,\left[w, x^{4} . v\right]\right]+\left[x^{1} . v,\left[x^{1} . v, x^{4} . v\right]\right]- \\
16\left[x^{1} . w,\left[w, x^{3} . v\right]\right]-48\left[x^{1} . w,\left[x^{1} . v, x^{2} . w\right]\right]-\left[x^{2} . v,\left[v, x^{4} . v\right]\right]- \\
12\left[x^{2} . v,\left[w, x^{2} . w\right]\right]-2\left[x^{2} . v,\left[w, x^{3} . v\right]\right]-18\left[x^{2} . v,\left[x^{1} . v, x^{2} . w\right]\right]\end{array}$ \\
\hline 3 & 0 & $2\left[x^{3} . v,\left[v, x^{3} . v\right]\right]$ & $\begin{array}{l}3\left[x^{1} . v,\left[x^{1} . v, x^{4} . v\right]\right]+9\left[x^{2} \cdot v,\left[v, x^{4} . v\right]\right]-72\left[x^{2} . v,\left[w, x^{2} . w\right]\right]- \\
12\left[x^{2} . v,\left[w, x^{3} . v\right]\right]+36\left[x^{2} . v,\left[x^{1} . v, x^{2} . w\right]\right]\end{array}$ \\
\hline 3 & 0 & $16\left[x^{3} . v,\left[w, x^{1} . w\right]\right]$ & 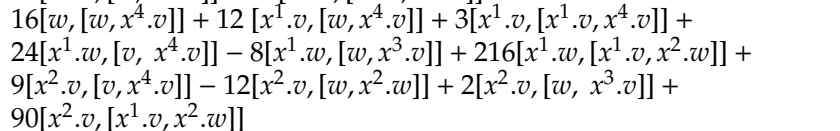 \\
\hline 3 & 0 & $4\left[x^{3} . v,\left[w, x^{2} . v\right]\right]$ & 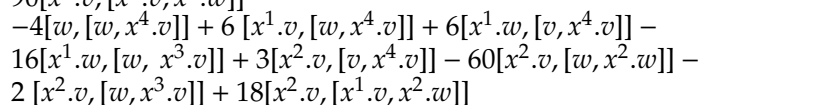 \\
\hline 3 & 0 & $4\left[x^{4} . v,\left[v, x^{1} . w\right]\right]$ & $-2\left[x^{1} . w,\left[v, x^{4} . v\right]\right]-\left[x^{2} . v,\left[v, x^{4} . v\right]\right]+24\left[x^{2} . v,\left[w, x^{2} . w\right]\right]$ \\
\hline 3 & 0 & {$\left[x^{4} . v,\left[v, x^{2} . v\right]\right]$} & 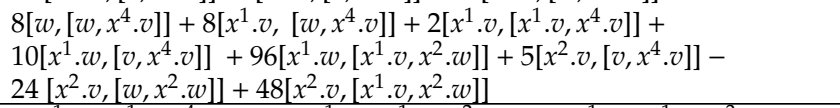 \\
\hline 3 & 1 & {$\left[x^{2} . w,\left[v, x^{4} . v\right]\right]$} & $\begin{array}{l}-\left[x^{1} \cdot w,\left[x^{1} \cdot v, x^{4} \cdot v\right]\right]+16\left[x^{1} \cdot w,\left[x^{1} \cdot w, x^{2} \cdot w\right]\right]-4\left[x^{1} \cdot w,\left[x^{1} \cdot w, x^{3} \cdot v\right]\right]+ \\
4\left[x^{2} \cdot v,\left[x^{1} \cdot w, x^{2} \cdot w\right]\right]\end{array}$ \\
\hline 3 & 1 & {$\left[x^{2} . w,\left[w, x^{2} . w\right]\right]$} & {$\left[x^{1} . w,\left[x^{1} . w, x^{2} . w\right]\right]$} \\
\hline 3 & 1 & $24\left[x^{2} . w,\left[x^{1} \cdot v, x^{2} \cdot w\right]\right]$ & $\begin{array}{l}2\left[x^{1} \cdot w,\left[w, x^{4} \cdot v\right]\right]+6\left[x^{1} \cdot w,\left[x^{1} \cdot v, x^{4} \cdot v\right]\right]-8\left[x^{1} \cdot w,\left[x^{1} \cdot w, x^{2} \cdot w\right]\right]+ \\
16\left[x^{1} \cdot w,\left[x^{1} \cdot w, x^{3} \cdot v\right]\right]+\left[x^{2} \cdot v,\left[w, x^{4} \cdot v\right]\right]+2\left[x^{2} \cdot v,\left[x^{1} \cdot v, x^{4} \cdot v\right]\right]- \\
20\left[x^{2} \cdot v,\left[x^{1} \cdot w, x^{2} \cdot v\right]\right]+4\left[x^{2} \cdot v,\left[x^{1} \cdot w, x^{3} \cdot v\right]\right]-8\left[x^{2} \cdot w,\left[w, x^{3} . v\right]\right]\end{array}$ \\
\hline 3 & 1 & $4\left[x^{3} . v,\left[v, x^{4} . v\right]\right]$ & 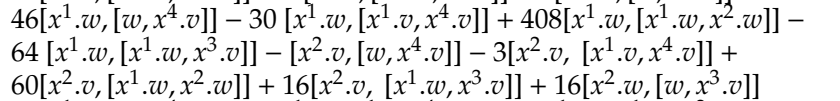 \\
\hline 3 & 1 & $32\left[x^{3} . v,\left[w, x^{2} . w\right]\right]$ & 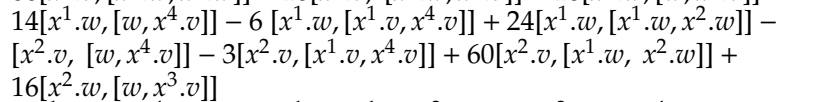 \\
\hline 3 & 1 & $4\left[x^{3} . v,\left[w, x^{3} . v\right]\right]$ & $\begin{array}{l}6\left[x^{1} \cdot w,\left[w, x^{4} \cdot v\right]\right]+72\left[x^{1} \cdot w,\left[x^{1} \cdot w, x^{2} \cdot w\right]\right]+3\left[x^{2} \cdot v,\left[w, x^{4} \cdot v\right]\right]- \\
36\left[x^{2} \cdot v,\left[x^{1} \cdot w, x^{2} \cdot w\right]\right]+12\left[x^{2} \cdot v,\left[x^{1} \cdot w, x^{3} \cdot v\right]\right]-24\left[x^{2} \cdot w,\left[w, x^{3} \cdot v\right]\right]\end{array}$ \\
\hline 3 & 1 & $48\left[x^{3} . v,\left[x^{1} . v, x^{2} . w\right]\right]$ & 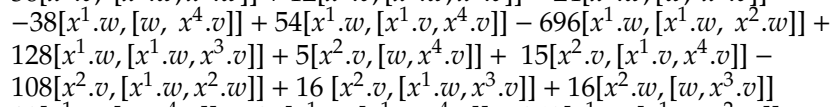 \\
\hline 3 & 1 & $16\left[x^{4} . v,\left[v, x^{2} . w\right]\right]$ & $\begin{array}{l}18\left[x^{1} \cdot w,\left[w, x^{4} \cdot v\right]\right]-26\left[x^{1} \cdot w,\left[x^{1} \cdot v, x^{4} \cdot v\right]\right]+360\left[x^{1} \cdot w,\left[x^{1} \cdot w, x^{2} \cdot w\right]\right]- \\
96\left[x^{1} \cdot w,\left[x^{1} \cdot w, x^{3} \cdot v\right]\right]+\left[x^{2} \cdot v,\left[w, x^{4} \cdot v\right]\right]-5\left[x^{2} \cdot v,\left[x^{1} \cdot v, x^{4} \cdot v\right]\right]+ \\
132\left[x^{2} \cdot v,\left[x^{1} \cdot w, x^{2} \cdot w\right]\right]+48\left[x^{2} \cdot w,\left[w, x^{3} \cdot v\right]\right]\end{array}$ \\
\hline 3 & 1 & {$\left[x^{4} . v,\left[v, x^{3} . v\right]\right]$} & 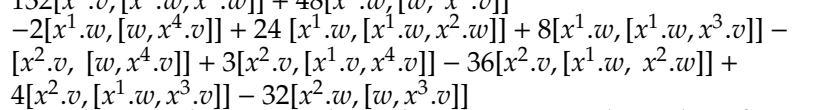 \\
\hline 3 & 1 & $96\left[x^{4} . v,\left[w, x^{1} . w\right]\right]$ & 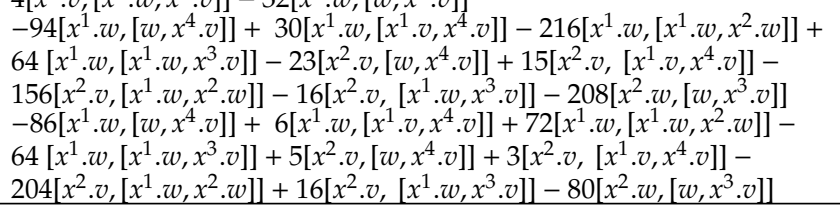 \\
\hline 3 & 2 & {$\left[x^{2} . w,\left[x^{1} . w, x^{2} . w\right]\right]$} & 0 \\
\hline 3 & 2 & $8\left[x^{3} . v,\left[w, x^{4} . v\right]\right]$ & $\begin{array}{l}-72\left[x^{1} \cdot w,\left[x^{1} \cdot w, x^{4} \cdot v\right]\right]+9\left[x^{2} \cdot v,\left[x^{2} \cdot v, x^{4} \cdot v\right]\right]-240\left[x^{2} \cdot w,\left[w, x^{4} \cdot v\right]\right]+ \\
36\left[x^{2} \cdot w,\left[x^{1} \cdot v, x^{4} \cdot v\right]\right]-240\left[x^{2} \cdot w,\left[x^{1} \cdot v, x^{3} \cdot v\right]\right]\end{array}$ \\
\hline 3 & 2 & $2\left[x^{3} . v,\left[x^{1} . v, x^{4} . v\right]\right]$ & $\begin{array}{l}-24\left[x^{1} \cdot w,\left[x^{1} \cdot w, x^{4} \cdot v\right]\right]+3\left[x^{2} \cdot v,\left[x^{2} \cdot v, x^{4} \cdot v\right]\right]-48\left[x^{2} . w,\left[w, x^{4} \cdot v\right]\right]+ \\
12\left[x^{2} \cdot w,\left[x^{1} \cdot v, x^{4} . v\right]\right]-48\left[x^{2} \cdot w,\left[x^{1} \cdot w, x^{3} \cdot v\right]\right]\end{array}$ \\
\hline
\end{tabular}




\begin{tabular}{|c|c|c|c|}
\hline 1 & $\mathrm{~m}$ & $\operatorname{LM}(b)$ for $b \in \mathrm{B}$ & $b-\operatorname{LM}(b)$ \\
\hline 3 & 2 & $32\left[x^{3} \cdot v,\left[x^{1} \cdot w, x^{2} \cdot w\right]\right]$ & $\begin{array}{l}24\left[x^{1} \cdot w,\left[x^{1} \cdot w, x^{4} \cdot v\right]\right]+8\left[x^{2} \cdot v,\left[x^{1} \cdot w, x^{4} \cdot v\right]\right]-\left[x^{2} \cdot v,\left[x^{2} \cdot v, x^{4} \cdot v\right]\right]+ \\
64\left[x^{2} \cdot w,\left[w, x^{4} \cdot v\right]\right]+12\left[x^{2} \cdot w,\left[x^{1} \cdot v, x^{4} \cdot v\right]\right]+112\left[x^{2} \cdot w,\left[x^{1} \cdot w, x^{3} \cdot v\right]\right]\end{array}$ \\
\hline 3 & 2 & $16\left[x^{3} . v,\left[x^{1} . w, x^{3} . v\right]\right]$ & $\begin{array}{l}72\left[x^{1} \cdot w,\left[x^{1} \cdot w, x^{4} \cdot v\right]\right]-9\left[x^{2} \cdot v,\left[x^{2} \cdot v, x^{4} \cdot v\right]\right]+192\left[x^{2} \cdot w,\left[w, x^{4} \cdot v\right]\right]- \\
36\left[x^{2} \cdot w,\left[x^{1} \cdot v, x^{4} \cdot v\right]\right]+144\left[x^{2} \cdot w,\left[x^{1} \cdot w, x^{3} \cdot v\right]\right]\end{array}$ \\
\hline 3 & 2 & {$\left[x^{4} . v,\left[v, x^{4} . v\right]\right]$} & $\begin{array}{l}30\left[x^{1} \cdot w,\left[x^{1} \cdot w, x^{4} \cdot v\right]\right]+9\left[x^{2} \cdot v,\left[x^{1} \cdot w, x^{4} \cdot v\right]\right]+24\left[x^{2} \cdot w,\left[w, x^{4} \cdot v\right]\right]+ \\
12\left[x^{2} \cdot w,\left[x^{1} \cdot v, x^{4} \cdot v\right]\right]+96\left[x^{2} \cdot w,\left[x^{1} \cdot w, x^{3} \cdot v\right]\right]\end{array}$ \\
\hline 3 & 2 & $8\left[x^{4} . v,\left[w, x^{2} . w\right]\right]$ & $\begin{array}{l}-10\left[x^{1} \cdot w,\left[x^{1} \cdot w, x^{4} \cdot v\right]\right]-3\left[x^{2} . v,\left[x^{1} \cdot w, x^{4} . v\right]\right]+\left[x^{2} \cdot v,\left[x^{2} . v, x^{4} . v\right]\right]- \\
40\left[x^{2} \cdot w,\left[w, x^{4} \cdot v\right]\right]-48\left[x^{2} \cdot w,\left[x^{1} \cdot w, x^{3} . v\right]\right]\end{array}$ \\
\hline 3 & 2 & $8\left[x^{4} . v,\left[w, x^{3} . v\right]\right]$ & $\begin{array}{l}48\left[x^{1} \cdot w,\left[x^{1} \cdot w, x^{4} \cdot v\right]\right]+36\left[x^{2} \cdot v,\left[x^{1} \cdot w_{1} x^{4} \cdot v\right]\right]-3\left[x^{2} \cdot v,\left[x^{2} \cdot v, x^{4} \cdot v\right]\right]+ \\
144\left[x^{2} \cdot w,\left[w, x^{4} \cdot v\right]\right]+36\left[x^{2} \cdot w,\left[x^{1} \cdot v, x^{4} \cdot v\right]\right]+336\left[x^{2} \cdot v,\left[x^{1} \cdot w, x^{3} \cdot v\right]\right]\end{array}$ \\
\hline 3 & 2 & $8\left[x^{4} . v,\left[x^{1} . v, x^{2} . w\right]\right]$ & $\begin{array}{l}-20\left[x^{1} \cdot w,\left[x^{1} \cdot w, x^{4} \cdot v\right]\right]-2\left[x^{2} \cdot v,\left[x^{1} \cdot w, x^{4} \cdot v\right]\right]+\left[x^{2} \cdot v,\left[x^{2} \cdot v, x^{4} \cdot v\right]\right]- \\
32\left[x^{2} \cdot w,\left[w, x^{4} \cdot v\right]\right]-4\left[x^{2} \cdot w,\left[x^{1} \cdot v, x^{4} \cdot v\right]\right]-48\left[x^{2} \cdot w,\left[x^{1} \cdot v, x^{3} \cdot v\right]\right]\end{array}$ \\
\hline 3 & 3 & {$\left[x^{3} . v,\left[x^{2} . v, x^{4} . v\right]\right]$} & $-96\left[x^{2} \cdot w,\left[x^{1} \cdot w, x^{4} \cdot v\right]\right]-6\left[x^{2} \cdot w,\left[x^{2} . v, x^{4} \cdot v\right]\right]-8\left[x^{3} \cdot v,\left[x^{1} \cdot w, x^{4} . v\right]\right]$ \\
\hline 3 & 3 & {$\left[x^{4} . v,\left[w, x^{4} . v\right]\right]$} & $54\left[x^{2} \cdot w,\left[x^{1} \cdot w, x^{4} \cdot v\right]\right]+6\left[x^{2} \cdot w,\left[x^{2} \cdot v, x^{4} \cdot v\right]\right]+7\left[x^{3} \cdot v,\left[x^{1} \cdot w, x^{4} \cdot v\right]\right]$ \\
\hline 3 & 3 & {$\left[x^{4} . v,\left[x^{1} . v, x^{4} . v\right]\right]$} & $192\left[x^{2} . w,\left[x^{1} . w, x^{4} . v\right]\right]+12\left[x^{2} . w,\left[x^{2} . v, x^{4} . v\right]\right]+16\left[x^{3} . v,\left[x^{1} . w, x^{4} . v\right]\right]$ \\
\hline 3 & 3 & $24\left[x^{4} \cdot v,\left[x^{1} \cdot w, x^{2} \cdot w\right]\right]$ & $66\left[x^{2} \cdot w,\left[x^{1} \cdot w, x^{4} \cdot v\right]\right]+6\left[x^{2} \cdot w,\left[x^{2} \cdot v, x^{4} \cdot v\right]\right]+5\left[x^{3} \cdot v,\left[x^{1} \cdot w, x^{4} \cdot v\right]\right]$ \\
\hline 3 & 3 & $2\left[x^{4} . v,\left[x^{1} . w, x^{3} . v\right]\right]$ & $-90\left[x^{2} . w,\left[x^{1} . w, x^{4} . v\right]\right]-6\left[x^{2} . w,\left[x^{2} . v, x^{4} . v\right]\right]-7\left[x^{3} . v,\left[x^{1} . w, x^{4} . v\right]\right]$ \\
\hline 3 & 4 & $5\left[x^{3} . v,\left[x^{2} . w, x^{4} . v\right]\right]$ & $42\left[x^{2} . w,\left[x^{2} . w, x^{4} . v\right]\right]$ \\
\hline 3 & 4 & $5\left[x^{4} . v,\left[x^{1} . w, x^{4} . v\right]\right]$ & $-48\left[x^{2} . w,\left[x^{2} . w, x^{4} . v\right]\right]$ \\
\hline 3 & 4 & $5\left[x^{4} . v,\left[x^{2} . v, x^{4} . v\right]\right]$ & $336\left[x^{2} . w,\left[x^{2} . w, x^{4} . v\right]\right]$ \\
\hline 3 & 5 & {$\left[x^{4} . v,\left[x^{2} . w, x^{4} . v\right]\right]$} & 0 \\
\hline 4 & -6 & {$\left[v,\left[v,\left[v, x^{1} . w\right]\right]\right]$} & 0 \\
\hline 4 & -5 & {$\left[v,\left[v,\left[v, x^{3}, v\right]\right]\right]$} & $-21\left[v,\left[v,\left[v, x^{2} . w\right]\right]\right]$ \\
\hline 4 & -5 & $2\left[w,\left[v,\left[v, x^{1} . w\right]\right]\right]$ & $3\left[v,\left[v,\left[v, x^{2} . w\right]\right]\right]$ \\
\hline 4 & -5 & $3\left[x^{1} . v,\left[v,\left[v, x^{1} . w\right]\right]\right]$ & $11\left[v,\left[v,\left[v, x^{2} . w\right]\right]\right]$ \\
\hline 4 & -5 & $6\left[[v, w],\left[v, x^{1} . w\right]\right]$ & $-5\left[v,\left[v,\left[v, x^{2} . w\right]\right]\right]$ \\
\hline 4 & -5 & $3\left[[v, w],\left[v, x^{2} . v\right]\right]$ & $-14\left[v,\left[v,\left[v, x^{2} . w\right]\right]\right]$ \\
\hline 4 & -4 & $3648\left[w,\left[w,\left[v, x^{1} . w\right]\right]\right]$ & $-15\left[v,\left[v,\left[v, x^{4} . v\right]\right]\right]+768\left[w,\left[v,\left[v, x^{2} . w\right]\right]\right]+52\left[w,\left[v,\left[v, x^{3} . v\right]\right]\right]$ \\
\hline 4 & -4 & $1368\left[x^{1} . v,\left[v,\left[v, x^{2} . w\right]\right]\right]$ & $35\left[v,\left[v,\left[v, x^{4} . v\right]\right]\right]-3312\left[w,\left[v,\left[v, x^{2} . w\right]\right]\right]+132\left[w,\left[v,\left[v, x^{3} . v\right]\right]\right]$ \\
\hline 4 & -4 & $6\left[x^{1} . v,\left[v,\left[v, x^{3} . v\right]\right]\right]$ & $5\left[v,\left[v,\left[v, x^{4} . v\right]\right]\right]$ \\
\hline 4 & -4 & $5472\left[x^{1} . v,\left[w,\left[v, x^{1} . w\right]\right]\right]$ & $-175\left[v,\left[v,\left[v, x^{4} . v\right]\right]\right]+2880\left[w,\left[v,\left[v, x^{2} . w\right]\right]\right]-204\left[w,\left[v,\left[v, x^{3} . v\right]\right]\right]$ \\
\hline 4 & -4 & $456\left[x^{1} . w,\left[v,\left[v, x^{1} . w\right]\right]\right]$ & $27\left[v,\left[v,\left[v, x^{4} . v\right]\right]\right]-288\left[w,\left[v,\left[v, x^{2} . w\right]\right]\right]+28\left[w,\left[v,\left[v, x^{3} . v\right]\right]\right]$ \\
\hline 4 & -4 & $114\left[x^{2} . v,\left[v,\left[v, x^{1} . w\right]\right]\right]$ & $-\left[v,\left[v,\left[v, x^{4} . v\right]\right]\right]-192\left[w,\left[v,\left[v, x^{2} . w\right]\right]\right]-32\left[w,\left[v,\left[v, x^{3} . v\right]\right]\right]$ \\
\hline 4 & -4 & $5472\left[[v, w],\left[v, x^{2} . w\right]\right]$ & $-43\left[v,\left[v,\left[v, x^{4} . v\right]\right]\right]+3600\left[w,\left[v,\left[v, x^{2} . w\right]\right]\right]-84\left[w,\left[v,\left[v, x^{3} . v\right]\right]\right]$ \\
\hline 4 & -4 & $456\left[[v, w],\left[v, x^{3} . v\right]\right]$ & $-71\left[v,\left[v,\left[v, x^{4} . v\right]\right]\right]+504\left[w,\left[v,\left[v, x^{2} . w\right]\right]\right]+84\left[w,\left[v,\left[v, x^{3} . v\right]\right]\right]$ \\
\hline 4 & -4 & $304\left[[v, w],\left[w, x^{1} . w\right]\right]$ & $-5\left[v,\left[v,\left[v, x^{4} . v\right]\right]\right]+28\left[w,\left[v,\left[v, x^{2} . w\right]\right]\right]-8\left[w,\left[v,\left[v, x^{3} . v\right]\right]\right]$ \\
\hline 4 & -4 & $96\left[[v, w],\left[w, x^{2} . v\right]\right]$ & $-5\left[v,\left[v,\left[v, x^{4} . v\right]\right]\right]+48\left[w,\left[v,\left[v, x^{2} . w\right]\right]\right]-12\left[w,\left[v,\left[v, x^{3} . v\right]\right]\right]$ \\
\hline 4 & -4 & $342\left[\left[v, x^{1} . w\right],\left[v, x^{2} . v\right]\right]$ & $-35\left[v,\left[v,\left[v, x^{4} . v\right]\right]\right]+576\left[w,\left[v,\left[v, x^{2} . w\right]\right]\right]-132\left[w,\left[v,\left[v, x^{3} . v\right]\right]\right]$ \\
\hline 4 & -3 & $30\left[x^{1} . v,\left[x^{1} . v,\left[v, x^{2} . w\right]\right]\right]$ & $6\left[w,\left[v,\left[v, x^{4} . v\right]\right]\right]+672\left[w,\left[w,\left[v, x^{2} . w\right]\right]\right]-32\left[w,\left[w,\left[v, x^{3} . v\right]\right]\right]-$ \\
\hline & & & $\begin{array}{l}80\left[w,\left[w,\left[w, x^{2} . v\right]\right]\right]-3\left[x^{1} . v,\left[v,\left[v, x^{4} . v\right]\right]\right]-204\left[x^{1} . v,\left[w,\left[v, x^{2} . w\right]\right]\right]+ \\
34\left[x^{1} . v,\left[w,\left[v, x^{3} . v\right]\right]\right]+8\left[x^{1} . v,\left[w,\left[w, x^{2} . v\right]\right]\right]\end{array}$ \\
\hline 4 & -3 & $60\left[x^{1} . v,\left[x^{1} . v,\left[w, x^{1} . w\right]\right]\right]$ & $12\left[w,\left[v,\left[v, x^{4} . v\right]\right]\right]+204\left[w,\left[w,\left[v, x^{2} . w\right]\right]\right]+26\left[w,\left[w,\left[v, x^{3} . v\right]\right]\right]+$ \\
\hline & & & $\begin{array}{l}80\left[w,\left[w,\left[w, x^{2} . v\right]\right]\right]-6\left[x^{1} . v,\left[v,\left[v, x^{4} . v\right]\right]\right]+102\left[x^{1} . v,\left[w,\left[v, x^{2} . w\right]\right]\right]+ \\
13\left[x^{1} . v,\left[w,\left[v, x^{3} . v\right]\right]\right]-4\left[x^{1} . v,\left[w,\left[w, x^{2} . v\right]\right]\right]\end{array}$ \\
\hline 4 & -3 & $36\left[x^{1} . w,\left[v,\left[v, x^{2} . w\right]\right]\right]$ & $-15\left[w,\left[v,\left[v, x^{4} . v\right]\right]\right]+156\left[w,\left[w,\left[v, x^{2} . w\right]\right]\right]-46\left[w,\left[w,\left[v, x^{3} . v\right]\right]\right]-$ \\
\hline & & & $\begin{array}{l}112\left[w,\left[w,\left[w, x^{2} . v\right]\right]\right]+3\left[x^{1} . v,\left[v,\left[v, x^{4} . v\right]\right]\right]-162\left[x^{1} . v,\left[w,\left[v, x^{2} . w\right]\right]\right]- \\
9\left[x^{1} . v,\left[w,\left[v, x^{3} . v\right]\right]\right]+12\left[x^{1} . v,\left[w,\left[w, x^{2} . v\right]\right]\right]\end{array}$ \\
\hline 4 & -3 & $4\left[x^{1} . w,\left[v,\left[v, x^{3} . v\right]\right]\right]$ & $-18\left[w,\left[v,\left[v, x^{4} . v\right]\right]\right]+120\left[w,\left[w,\left[v, x^{2} . w\right]\right]\right]-52\left[w,\left[w,\left[v, x^{3} . v\right]\right]\right]-$ \\
\hline & & & $\begin{array}{l}160\left[w,\left[w,\left[w, x^{2} . v\right]\right]\right]+3\left[x^{1} . v,\left[v,\left[v, x^{4} . v\right]\right]\right]-84\left[x^{1} . v,\left[w,\left[v, x^{2} . w\right]\right]\right]+ \\
2\left[x^{1} \cdot v,\left[w,\left[v, x^{3} . v\right]\right]\right]+56\left[x^{1} . v,\left[w,\left[w, x^{2} . v\right]\right]\right]\end{array}$ \\
\hline 4 & -3 & $1440\left[x^{1} . w,\left[w,\left[v, x^{1} . w\right]\right]\right]$ & $294\left[w,\left[v,\left[v, x^{4} . v\right]\right]\right]-1992\left[w,\left[w,\left[v, x^{2} . w\right]\right]\right]+892\left[w,\left[w,\left[v, x^{3} . v\right]\right]\right]+$ \\
\hline & & & $\begin{array}{l}2560\left[w,\left[w,\left[w, x^{2} . v\right]\right]\right]-57\left[x^{1} . v,\left[v,\left[v, x^{4} . v\right]\right]\right]+2124\left[x^{1} . v,\left[w,\left[v, x^{2} . w\right]\right]\right]+ \\
66\left[x^{1} . v,\left[w,\left[v, x^{3} . v\right]\right]\right]-648\left[x^{1} . v,\left[w,\left[w, x^{2} . v\right]\right]\right]\end{array}$ \\
\hline 4 & -3 & $180\left[x^{2} . v,\left[v,\left[v, x^{2} . w\right]\right]\right]$ & $-156\left[w,\left[v,\left[v, x^{4} . v\right]\right]\right]+888\left[w,\left[w,\left[v, x^{2} . w\right]\right]\right]-188\left[w,\left[w,\left[v, x^{3} . v\right]\right]\right]-$ \\
\hline & & & $\begin{array}{l}320\left[w,\left[w,\left[w, x^{2} . v\right]\right]\right]+33\left[x^{1} . v,\left[v,\left[v, x^{4} . v\right]\right]\right]-1476\left[x^{1} . v,\left[w,\left[v, x^{2} . w\right]\right]\right]- \\
234\left[x^{1} . v,\left[w,\left[v, x^{3} . v\right]\right]+312\left[x^{1} . v,\left[w,\left[w, x^{2} . v\right]\right]\right]\right.\end{array}$ \\
\hline 4 & -3 & $5\left[x^{2} . v,\left[v,\left[v, x^{3} . v\right]\right]\right]$ & $-66\left[w,\left[v,\left[v, x^{4} . v\right]\right]\right]+468\left[w,\left[w,\left[v, x^{2} . w\right]\right]\right]-158\left[w,\left[w,\left[v, x^{3} . v\right]\right]\right]-$ \\
\hline & & & $\begin{array}{l}480\left[w,\left[w,\left[w, x^{2} . v\right]\right]\right]+18\left[x^{1} . v,\left[v,\left[v, x^{4} . v\right]\right]\right]-366\left[x^{1} . v,\left[w,\left[v, x^{2} . w\right]\right]\right]- \\
9\left[x^{1} . v,\left[w,\left[v, x^{3} . v\right]\right]\right]+132\left[x^{1} . v,\left[w,\left[w, x^{2} . v\right]\right]\right]\end{array}$ \\
\hline 4 & -3 & $360\left[x^{2} . v,\left[w,\left[v, x^{1} . w\right]\right]\right]$ & $186\left[w,\left[v,\left[v, x^{4} . v\right]\right]\right]-1308\left[w,\left[w,\left[v, x^{2} . w\right]\right]\right]+418\left[w,\left[w,\left[v, x^{3} . v\right]\right]\right]+$ \\
\hline & & & $1120\left[w,\left[w,\left[w, x^{2} . v\right]\right]\right]-48\left[x^{1} . v,\left[v,\left[v, x^{4} . v\right]\right]\right]+1386\left[x^{1} . v,\left[w,\left[v, x^{2} . w\right]\right]\right]+$ \\
\hline 4 & -3 & $18\left[x^{2} . w,\left[v,\left[v, x^{1} . w\right]\right]\right]$ & $-9\left[w,\left[v,\left[v, x^{4} . v\right]\right]\right]-60\left[w,\left[w,\left[v, x^{2} . w\right]\right]\right]-22\left[w,\left[w,\left[v, x^{3} . v\right]\right]\right]-$ \\
\hline & & & $\begin{array}{l}64\left[w,\left[w,\left[w, x^{2} . v\right]\right]\right]+3\left[x^{1} . v,\left[v,\left[v, x^{4} . v\right]\right]\right]-54\left[x^{1} . v,\left[w,\left[v, x^{2} . w\right]\right]\right]- \\
9\left[x^{1} . v,\left[w,\left[v, x^{3} . v\right]\right]\right]+12\left[x^{1} . v,\left[w,\left[w, x^{2} . v\right]\right]\right]\end{array}$ \\
\hline
\end{tabular}




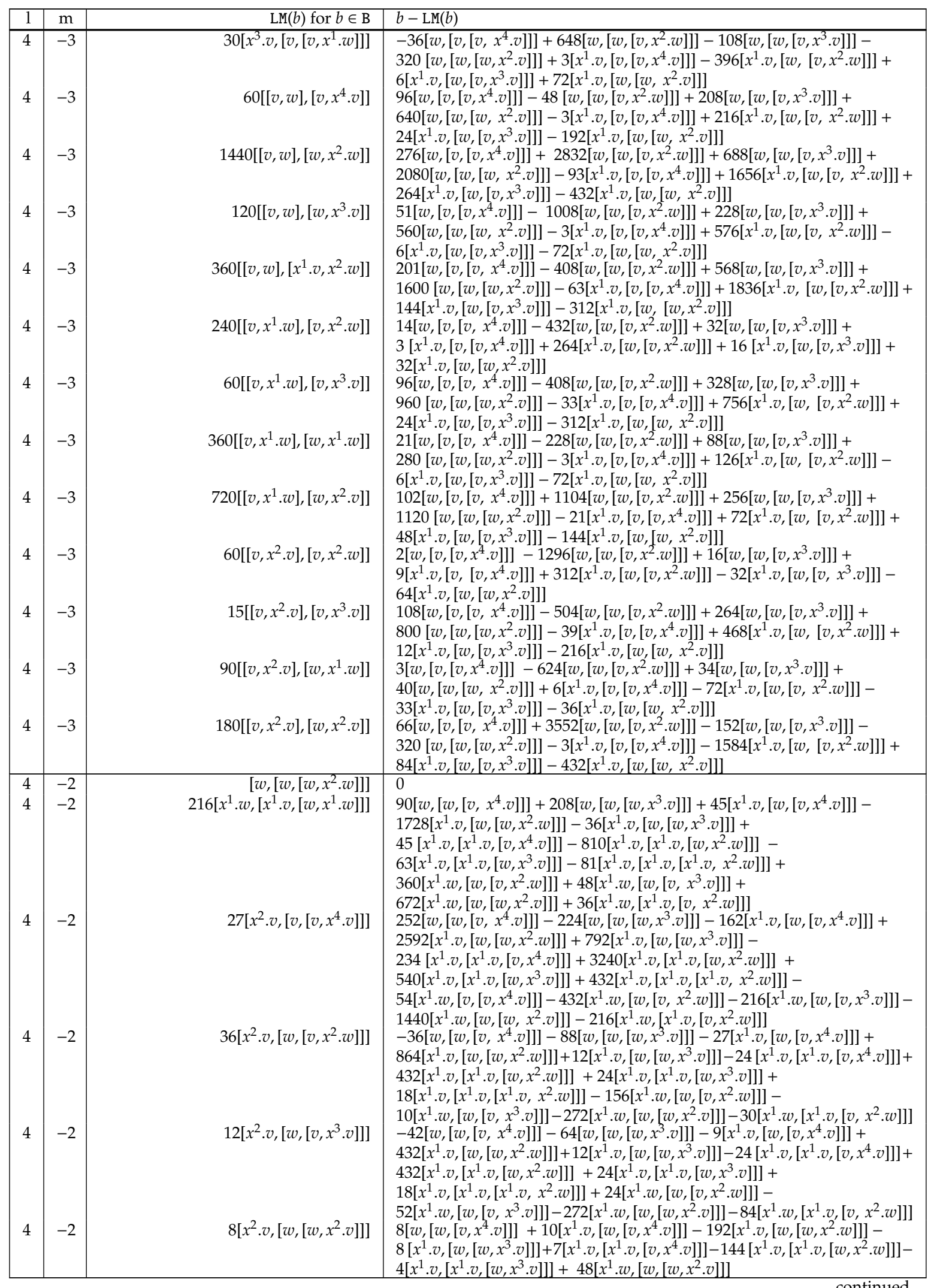


Appendix D. A Pseudo-Hall basis of $\mathfrak{g}$ for $d=3$

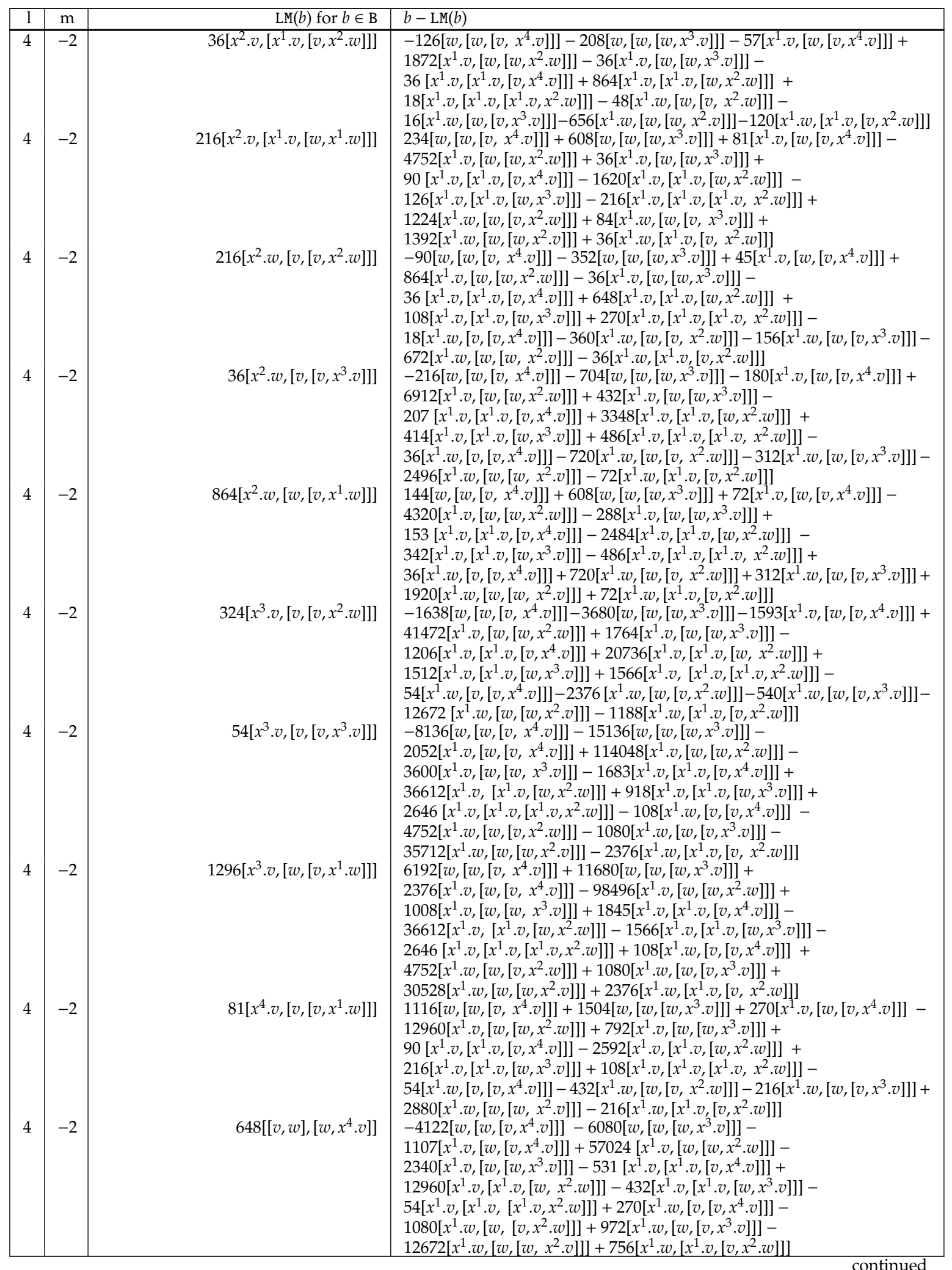




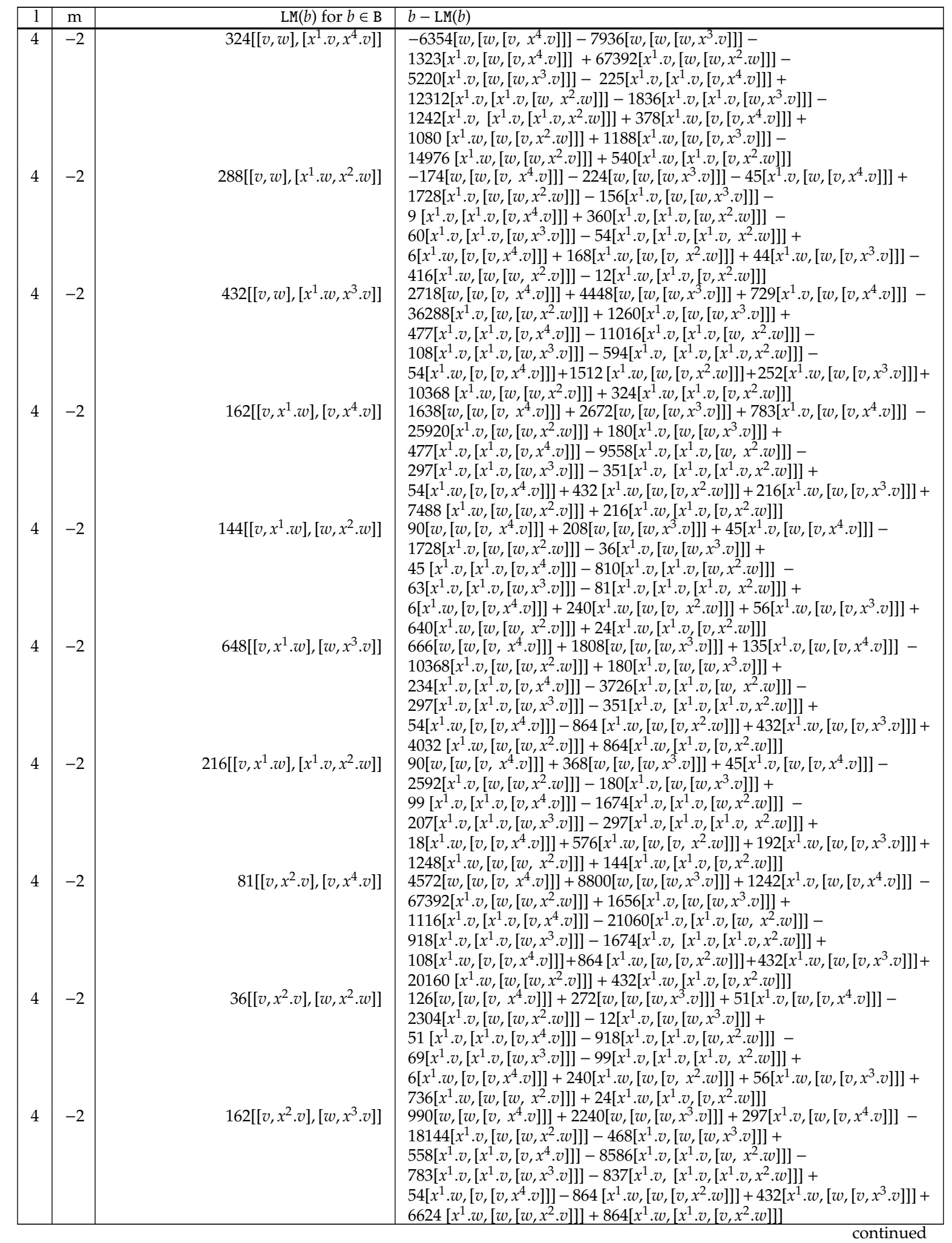




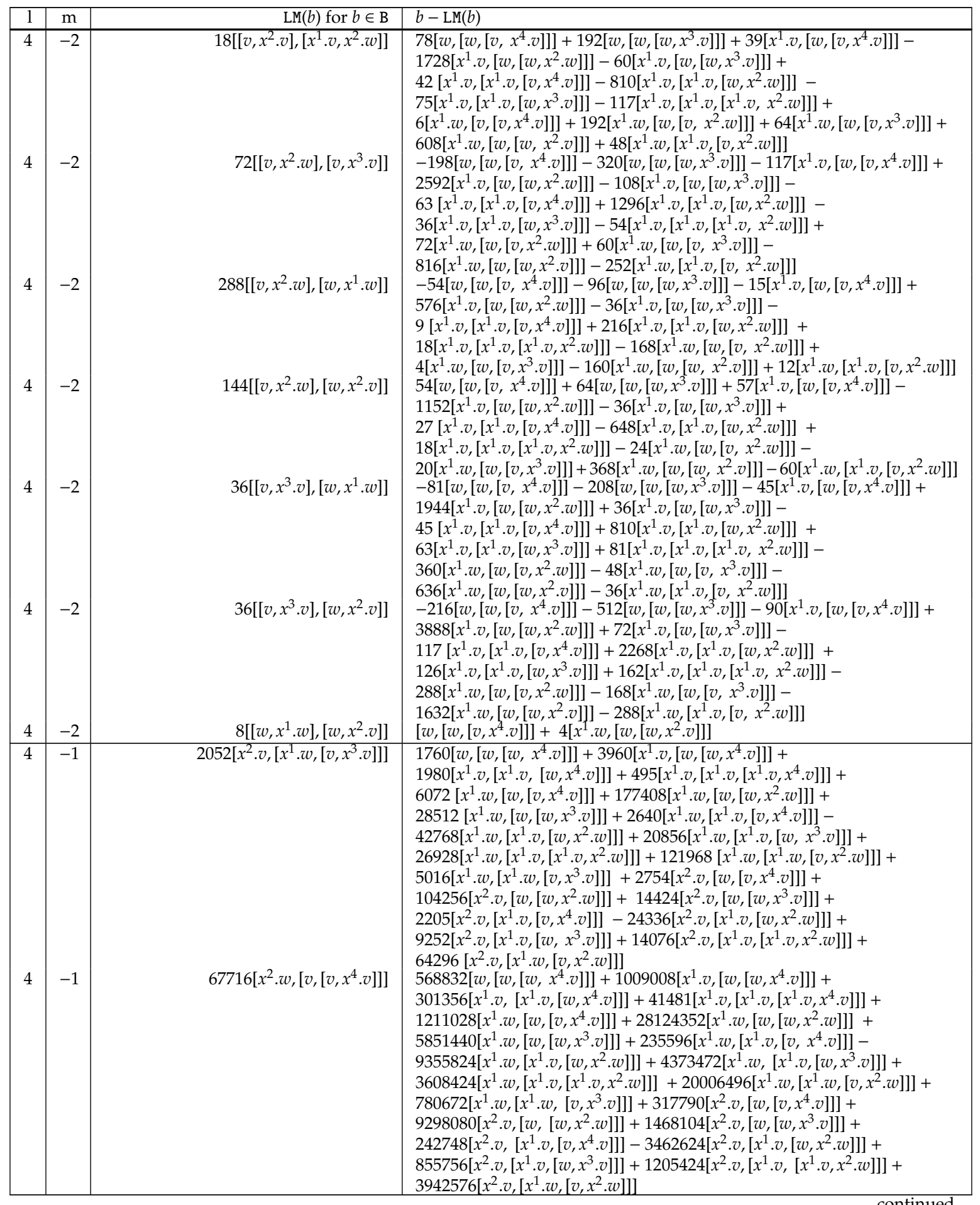




\begin{tabular}{|c|c|c|c|}
\hline 1 & $\mathrm{~m}$ & $\operatorname{LM}(b)$ for $b \in \mathrm{B}$ & $b-\operatorname{LM}(b)$ \\
\hline 4 & -1 & $180576\left[x^{2} . w,\left[w,\left[v, x^{2} . w\right]\right]\right]$ & 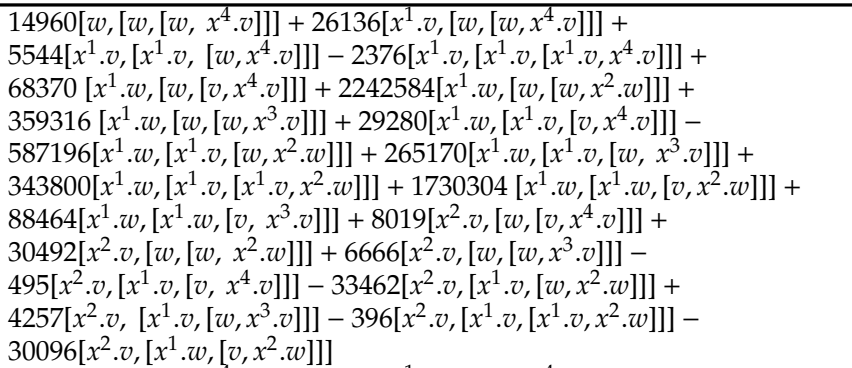 \\
\hline 4 & -1 & $14256\left[x^{2} . w,\left[w,\left[v, x^{3} . v\right]\right]\right]$ & 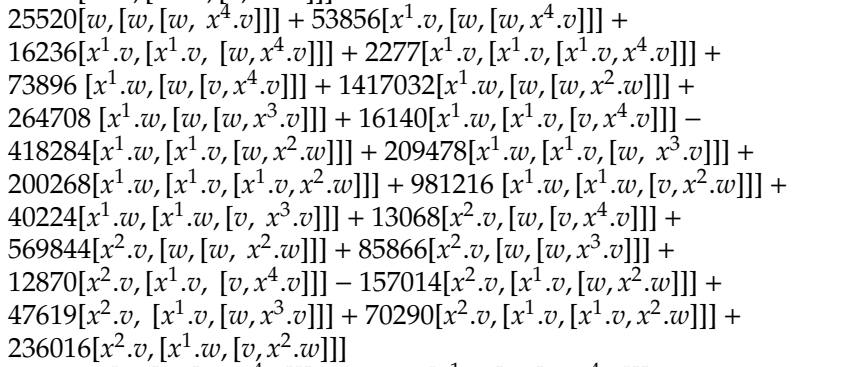 \\
\hline 4 & -1 & $270864\left[x^{2} . w,\left[w,\left[w, x^{2} . v\right]\right]\right]$ & 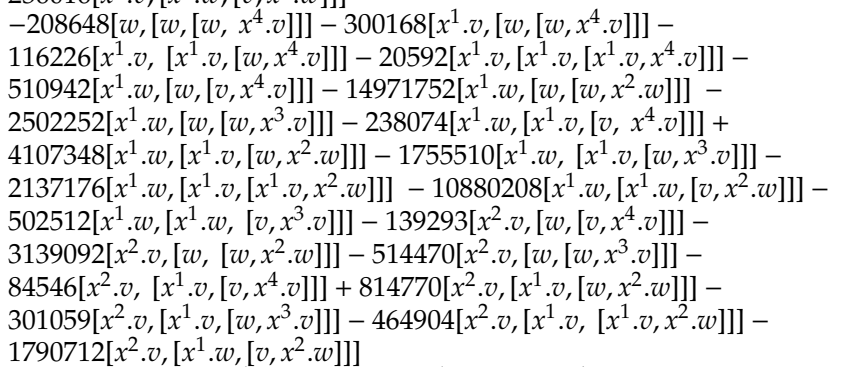 \\
\hline 4 & -1 & $24624\left[x^{2} . w,\left[x^{1} . v,\left[v, x^{2} . w\right]\right]\right]$ & 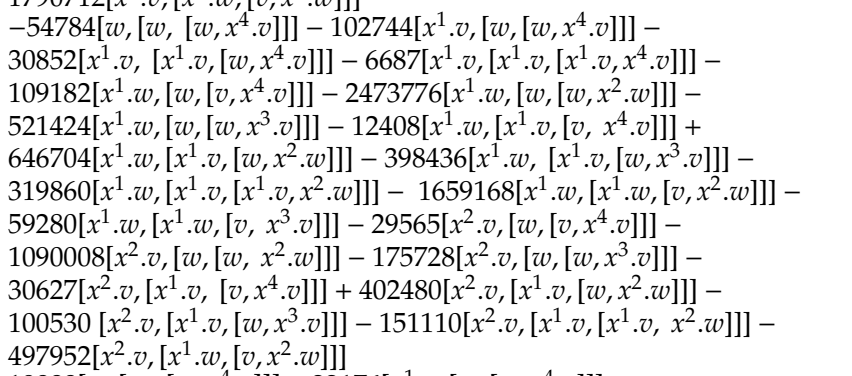 \\
\hline 4 & -1 & $180576\left[x^{2} . w,\left[x^{1} . v,\left[w, x^{1} . w\right]\right]\right]$ & 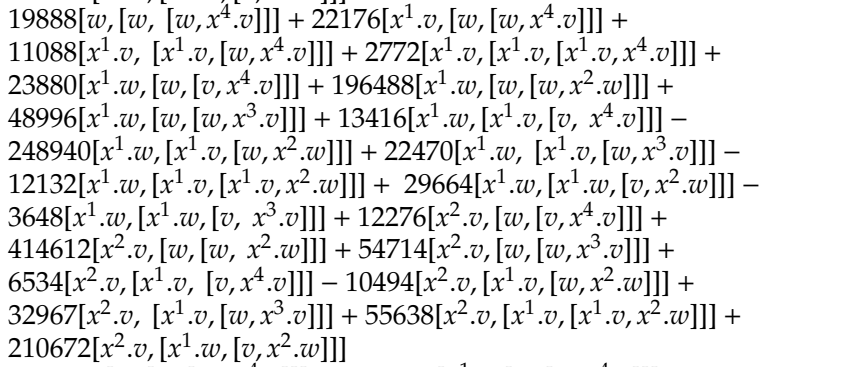 \\
\hline 4 & -1 & $33858\left[x^{3} . v,\left[v,\left[v, x^{4} . v\right]\right]\right]$ & 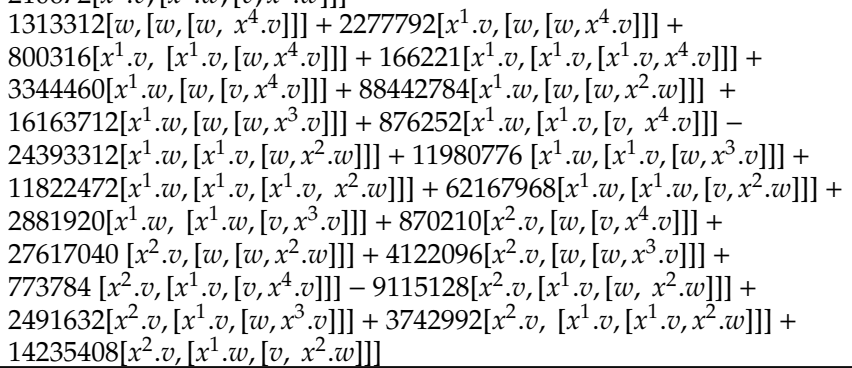 \\
\hline
\end{tabular}


Appendix D. A Pseudo-Hall basis of $\mathfrak{g}$ for $d=3$

\begin{tabular}{|c|c|c|c|}
\hline 1 & $\mathrm{~m}$ & $\mathrm{LM}(b)$ for $b \in \mathrm{B}$ & $b-\operatorname{LM}(b)$ \\
\hline 4 & $\overline{-1}$ & $270864\left[x^{3} . v,\left[w,\left[v, x^{2} . w\right]\right]\right]$ & 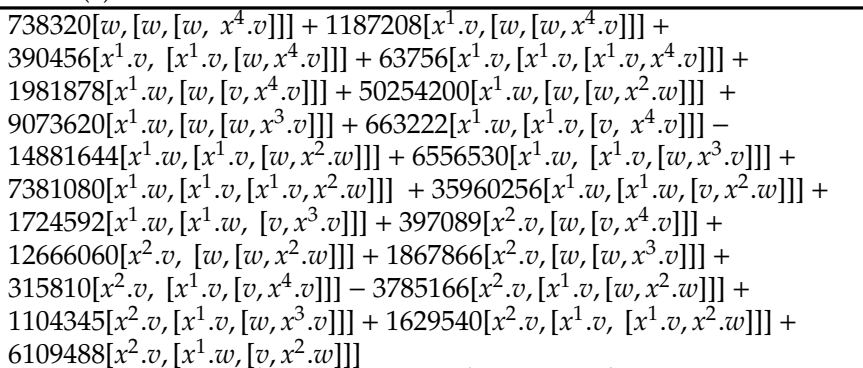 \\
\hline 4 & -1 & $45144\left[x^{3} . v,\left[w,\left[v, x^{3} . v\right]\right]\right]$ & 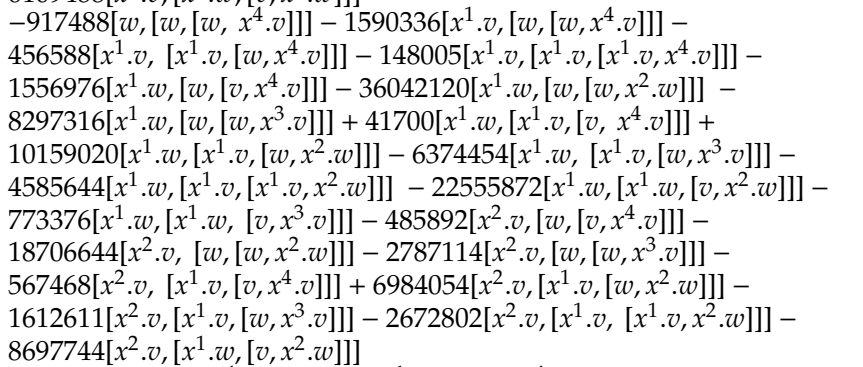 \\
\hline 4 & -1 & $2376\left[x^{3} . v,\left[w,\left[w, x^{2} . v\right]\right]\right]$ & 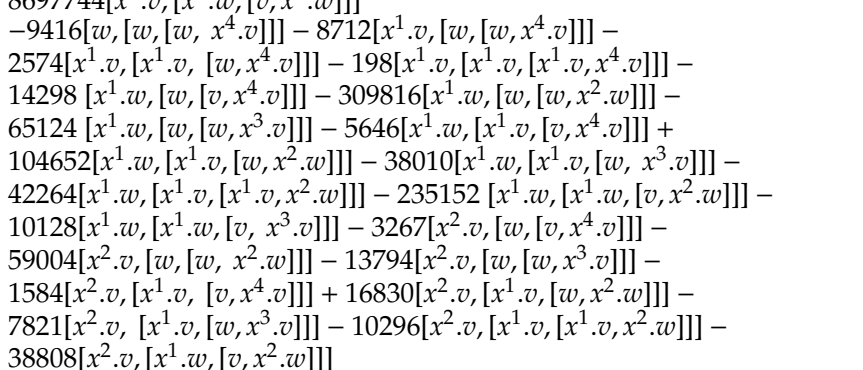 \\
\hline 4 & -1 & $135432\left[x^{3} . v,\left[x^{1} . v,\left[v, x^{2} . w\right]\right]\right]$ & 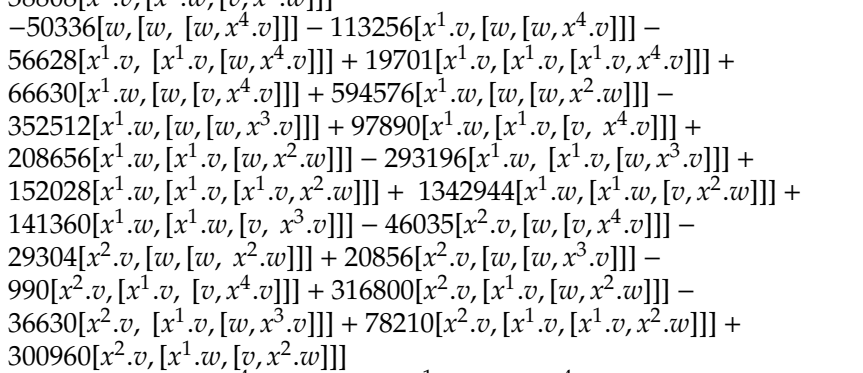 \\
\hline 4 & -1 & $24624\left[x^{3} . v,\left[x^{1} . v,\left[w, x^{1} . w\right]\right]\right]$ & 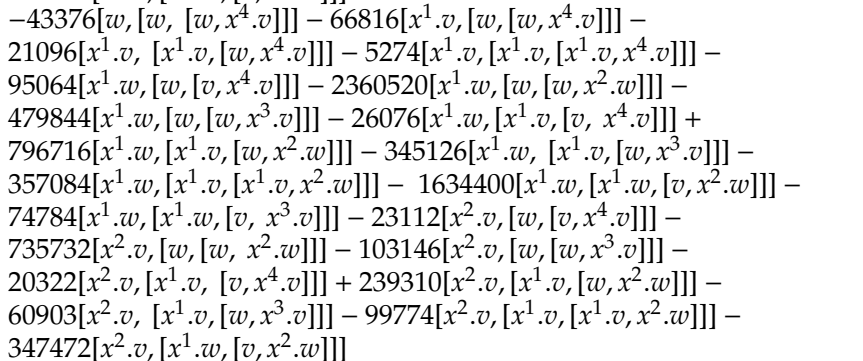 \\
\hline 4 & -1 & $203148\left[x^{4} . v,\left[v,\left[v, x^{2} . w\right]\right]\right]$ & 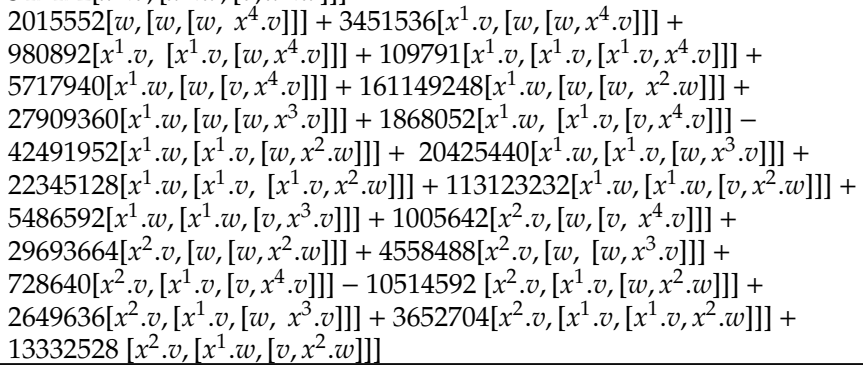 \\
\hline
\end{tabular}




\begin{tabular}{|c|c|c|c|}
\hline 1 & $\mathrm{~m}$ & $\mathrm{LM}(b)$ for $b \in \mathrm{B}$ & $b-\mathrm{LM}(b)$ \\
\hline 4 & $\begin{array}{ll}-1 \\
\end{array}$ & $33858\left[x^{4} . v,\left[v,\left[v, x^{3} . v\right]\right]\right]$ & 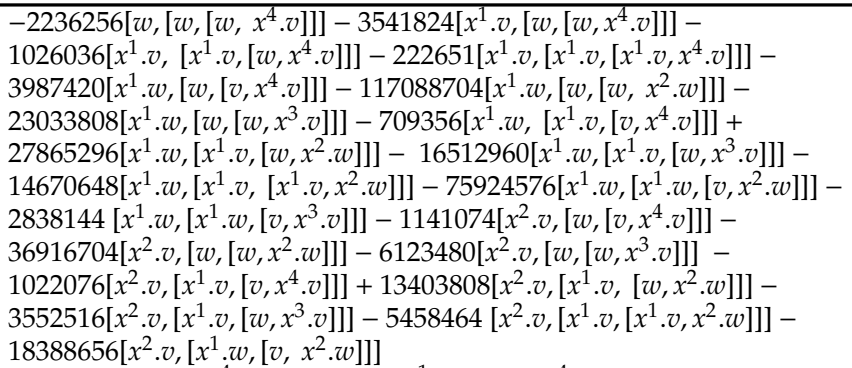 \\
\hline 4 & -1 & $812592\left[x^{4} . v,\left[w,\left[v, x^{1} . w\right]\right]\right]$ & 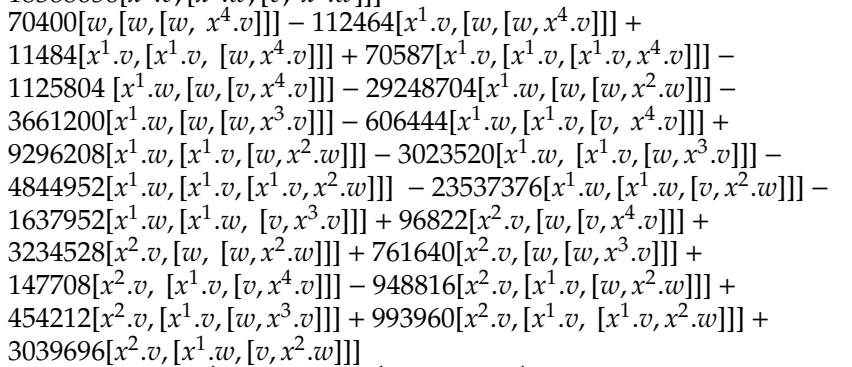 \\
\hline 4 & -1 & $2376\left[[v, w],\left[x^{1} . w, x^{4} . v\right]\right]$ & 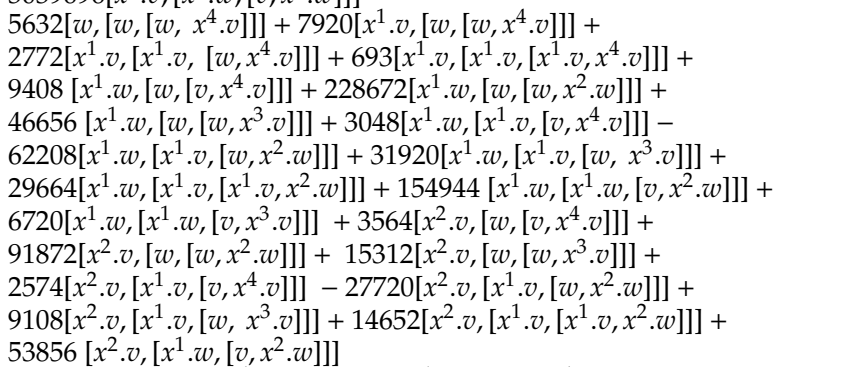 \\
\hline 4 & -1 & $9234\left[[v, w],\left[x^{2} . v, x^{4} . v\right]\right]$ & 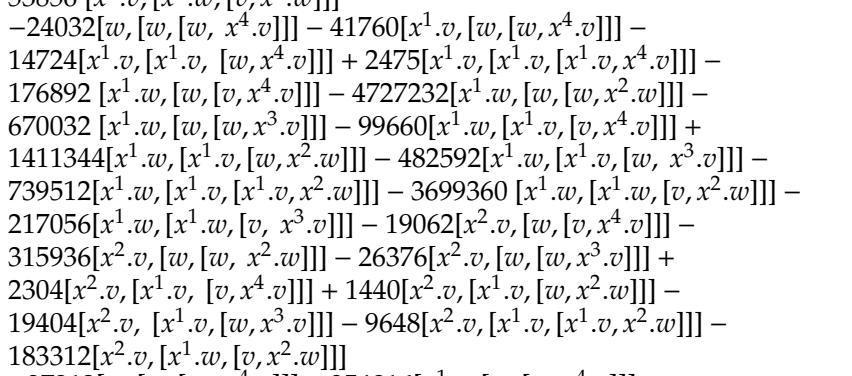 \\
\hline 4 & -1 & $812592\left[\left[v, x^{1} . w\right],\left[w, x^{4} . v\right]\right]$ & 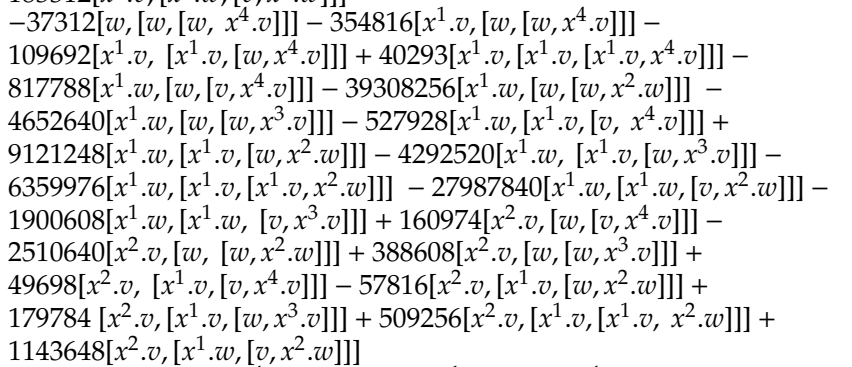 \\
\hline 4 & -1 & $21384\left[\left[v, x^{1} . w\right],\left[x^{1} . v, x^{4} . v\right]\right]$ & 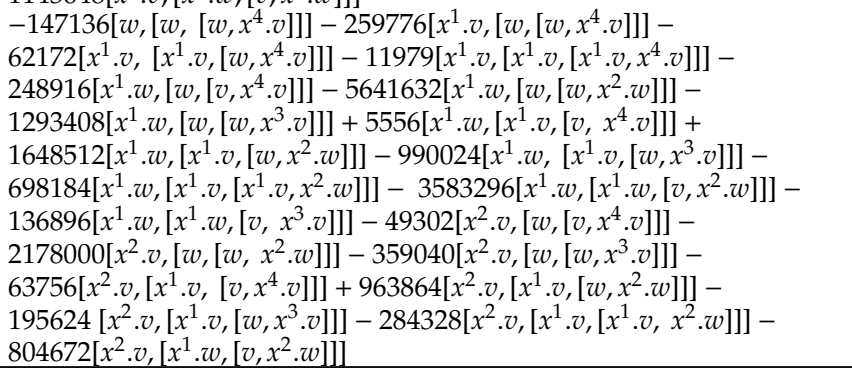 \\
\hline
\end{tabular}


Appendix D. A Pseudo-Hall basis of $\mathfrak{g}$ for $d=3$

\begin{tabular}{|c|c|c|c|}
\hline 1 & $\mathrm{~m}$ & $\mathrm{LM}(b)$ for $b \in \mathrm{B}$ & $b-\operatorname{LM}(b)$ \\
\hline 4 & $\overline{-1}$ & $1083456\left[\left[v, x^{1} \cdot w\right],\left[x^{1} \cdot w, x^{2} \cdot w\right]\right]$ & 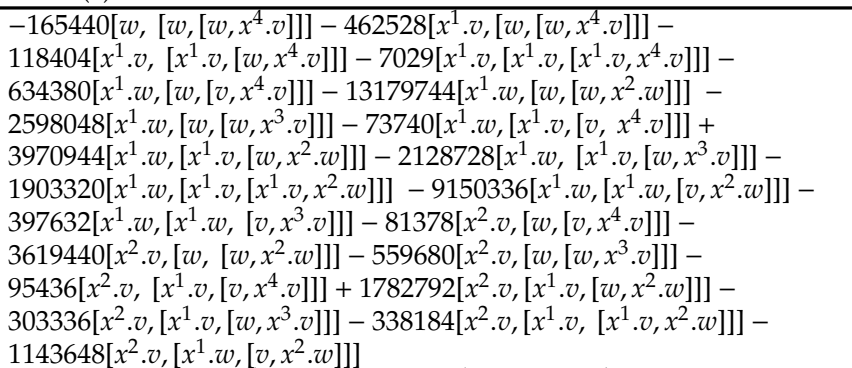 \\
\hline 4 & -1 & $1625184\left[\left[v, x^{1} . w\right],\left[x^{1} . w, x^{3} . v\right]\right]$ & 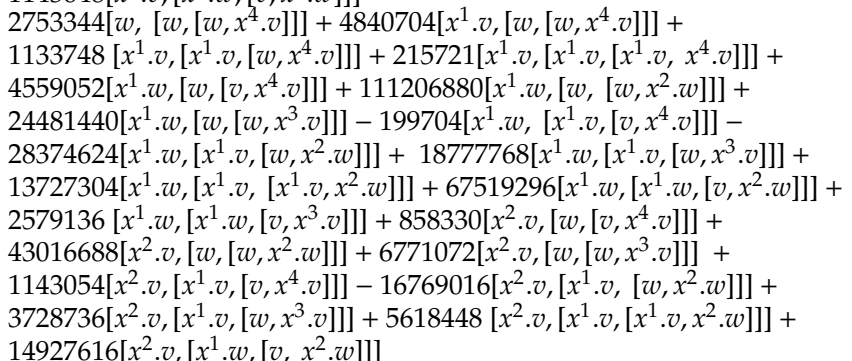 \\
\hline 4 & -1 & $203148\left[\left[v, x^{2} . v\right],\left[w, x^{4} . v\right]\right]$ & 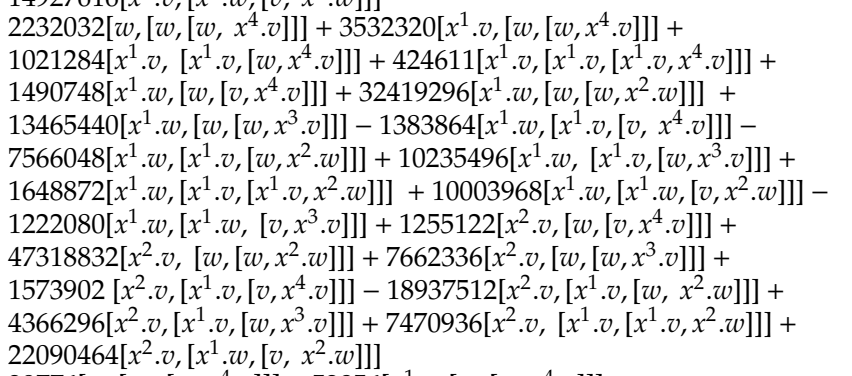 \\
\hline 4 & -1 & $5346\left[\left[v, x^{2} . v\right],\left[x^{1} . v, x^{4} . v\right]\right]$ & 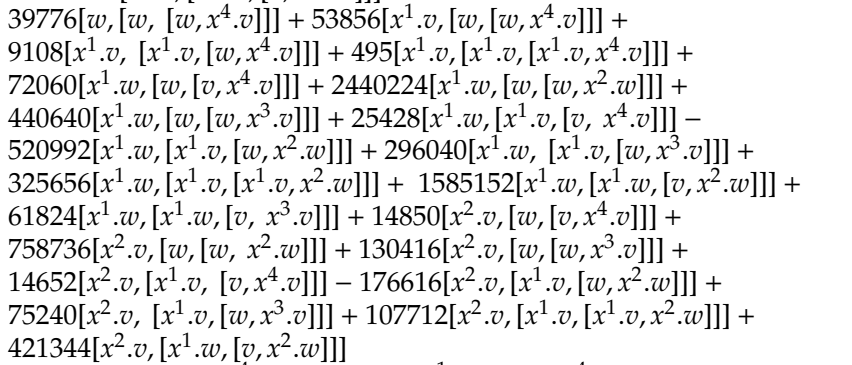 \\
\hline 4 & -1 & $90288\left[\left[v, x^{2} . v\right],\left[x^{1} . w, x^{2} . w\right]\right]$ & 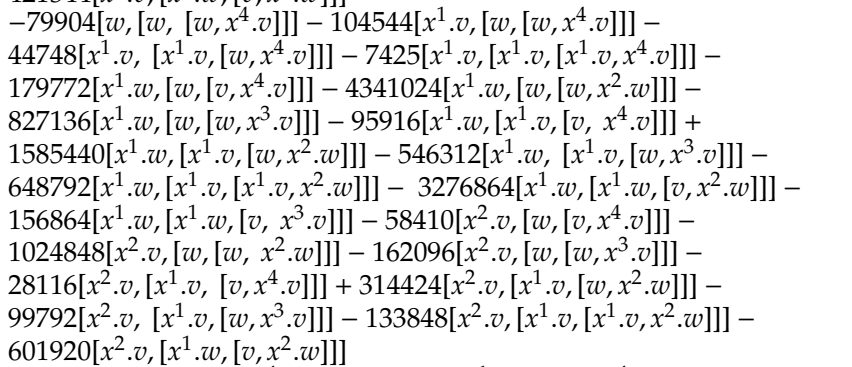 \\
\hline 4 & -1 & $406296\left[\left[v, x^{2} . v\right],\left[x^{1} . w, x^{3} . v\right]\right]$ & 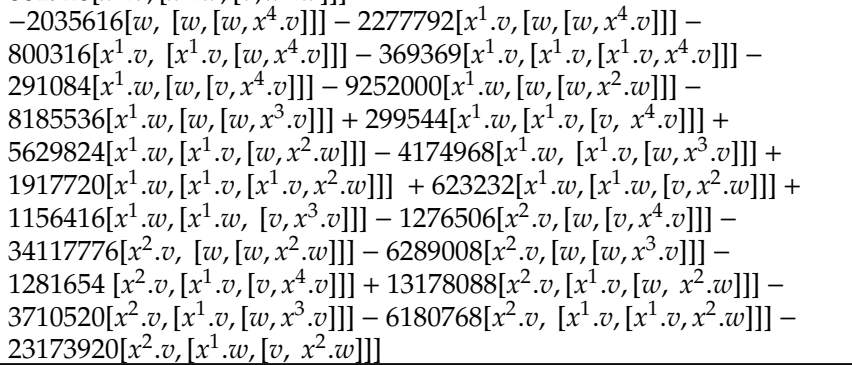 \\
\hline
\end{tabular}




\begin{tabular}{|c|c|c|c|}
\hline 1 & $\mathrm{~m}$ & $\operatorname{LM}(b)$ for $b \in \mathrm{B}$ & $b-\operatorname{LM}(b)$ \\
\hline 4 & -1 & $22572\left[\left[v, x^{2} . w\right],\left[v, x^{4} . v\right]\right]$ & 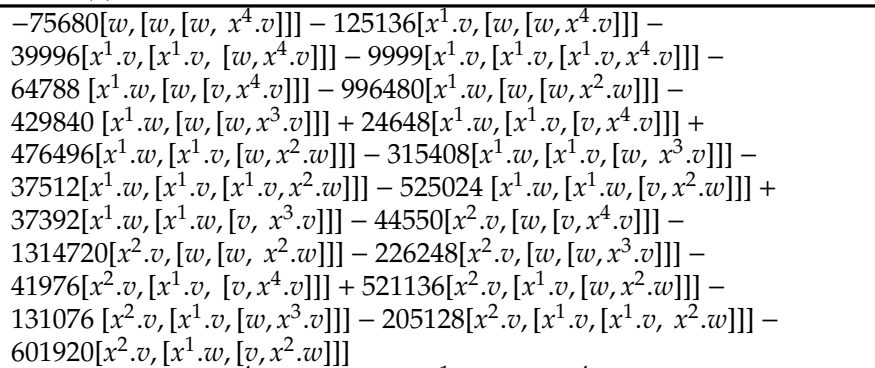 \\
\hline 4 & -1 & $180576\left[\left[v, x^{2} . w\right],\left[w, x^{2} . w\right]\right]$ & 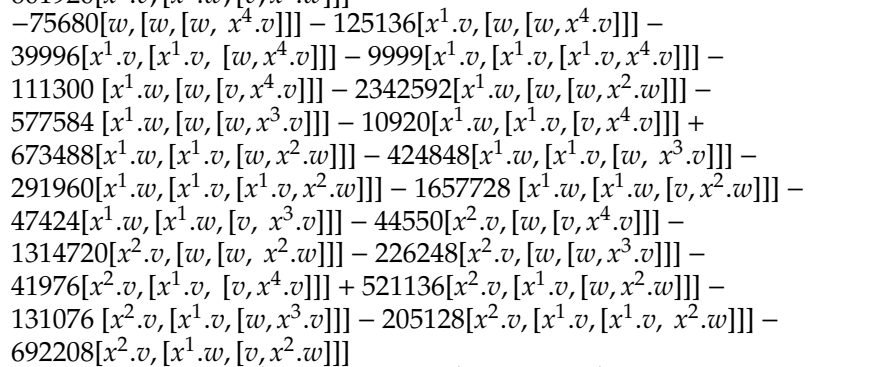 \\
\hline 4 & -1 & $270864\left[\left[v, x^{2} . w\right],\left[w, x^{3} . v\right]\right]$ & 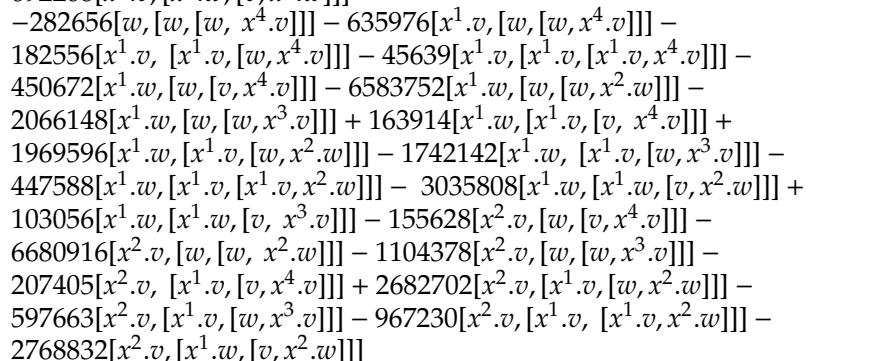 \\
\hline 4 & -1 & $270864\left[\left[v, x^{2} . w\right],\left[x^{1} . v, x^{2} . w\right]\right]$ & 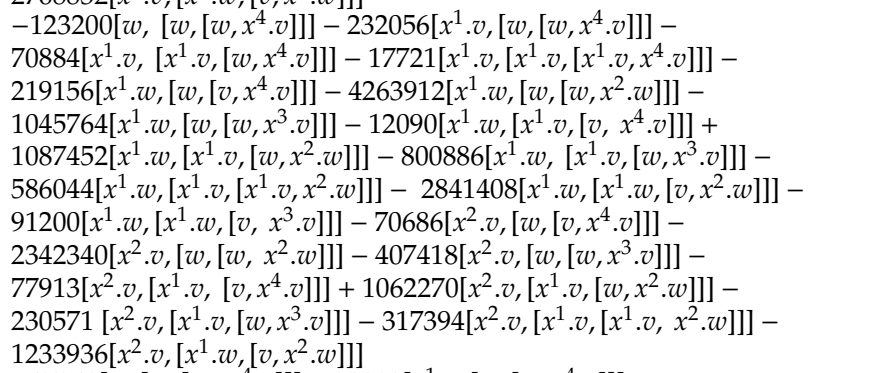 \\
\hline 4 & -1 & $5643\left[\left[v, x^{3} . v\right],\left[v, x^{4} . v\right]\right]$ & 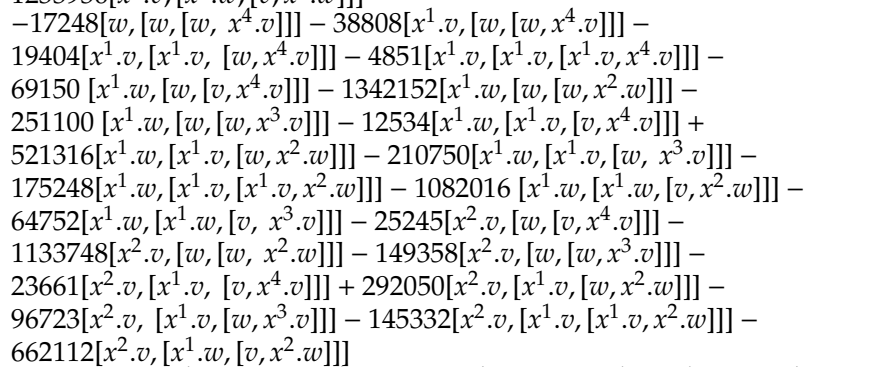 \\
\hline 4 & -1 & $1368\left[\left[v, x^{3} . v\right],\left[w, x^{2} . w\right]\right]$ & 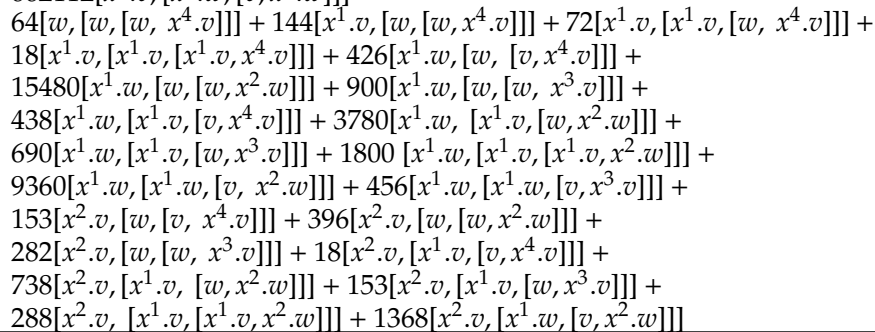 \\
\hline
\end{tabular}


Appendix D. A Pseudo-Hall basis of $\mathfrak{g}$ for $d=3$

\begin{tabular}{|c|c|c|c|}
\hline 1 & $\mathrm{~m}$ & $\mathrm{LM}(b)$ for $b \in \mathrm{B}$ & $b-\operatorname{LM}(b)$ \\
\hline 4 & $\overline{-1}$ & $45144\left[\left[v, x^{3} \cdot v\right],\left[w, x^{3} \cdot v\right]\right]$ & 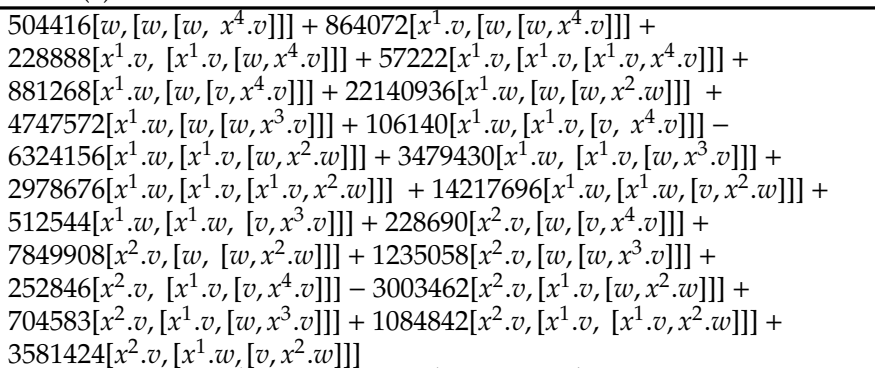 \\
\hline 4 & -1 & $135432\left[\left[v, x^{3} . v\right],\left[x^{1} . v, x^{2} . w\right]\right]$ & 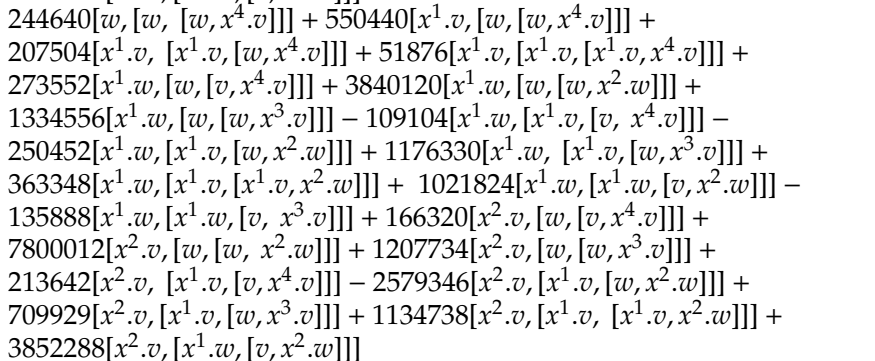 \\
\hline 4 & -1 & $11\left[\left[v, x^{4} . v\right],\left[w, x^{1} . w\right]\right]$ & 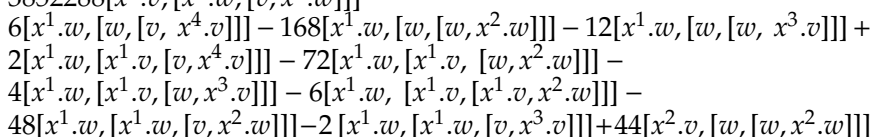 \\
\hline 4 & -1 & $11286\left[\left[v, x^{4} . v\right],\left[w, x^{2} . v\right]\right]$ & 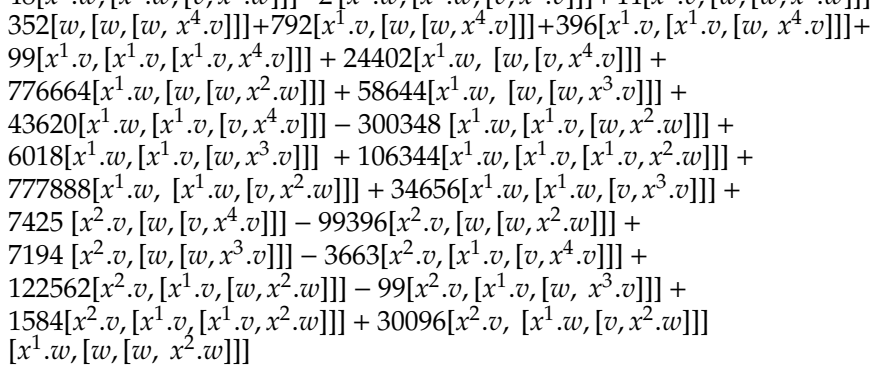 \\
\hline 4 & -1 & $60192\left[\left[w, x^{1} . w\right],\left[w, x^{3} . v\right]\right]$ & 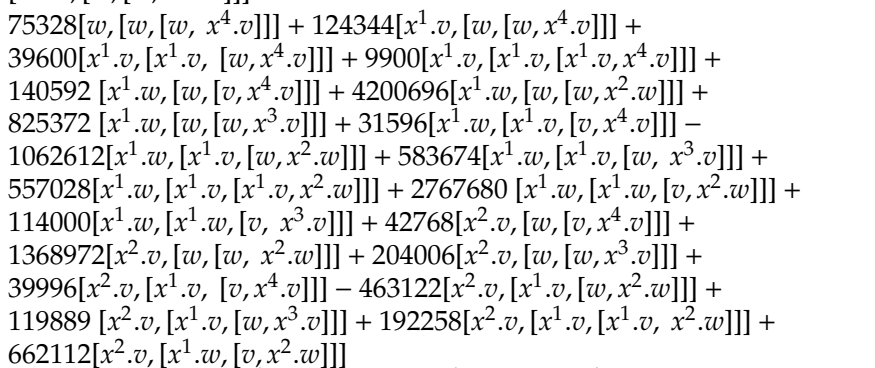 \\
\hline 4 & -1 & $541728\left[\left[w, x^{1} . w\right],\left[x^{1} . v, x^{2} . w\right]\right]$ & 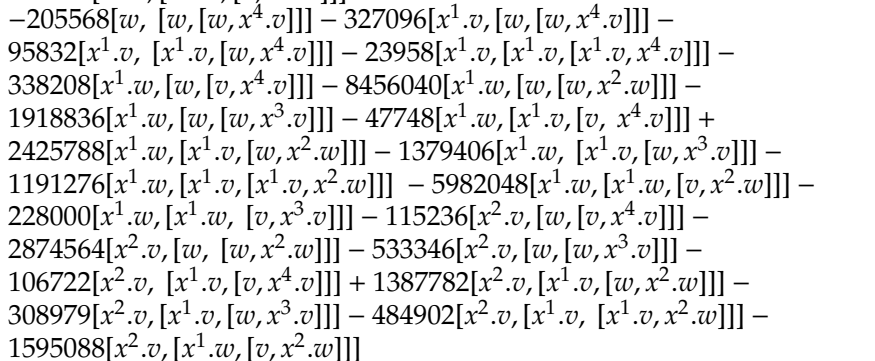 \\
\hline
\end{tabular}




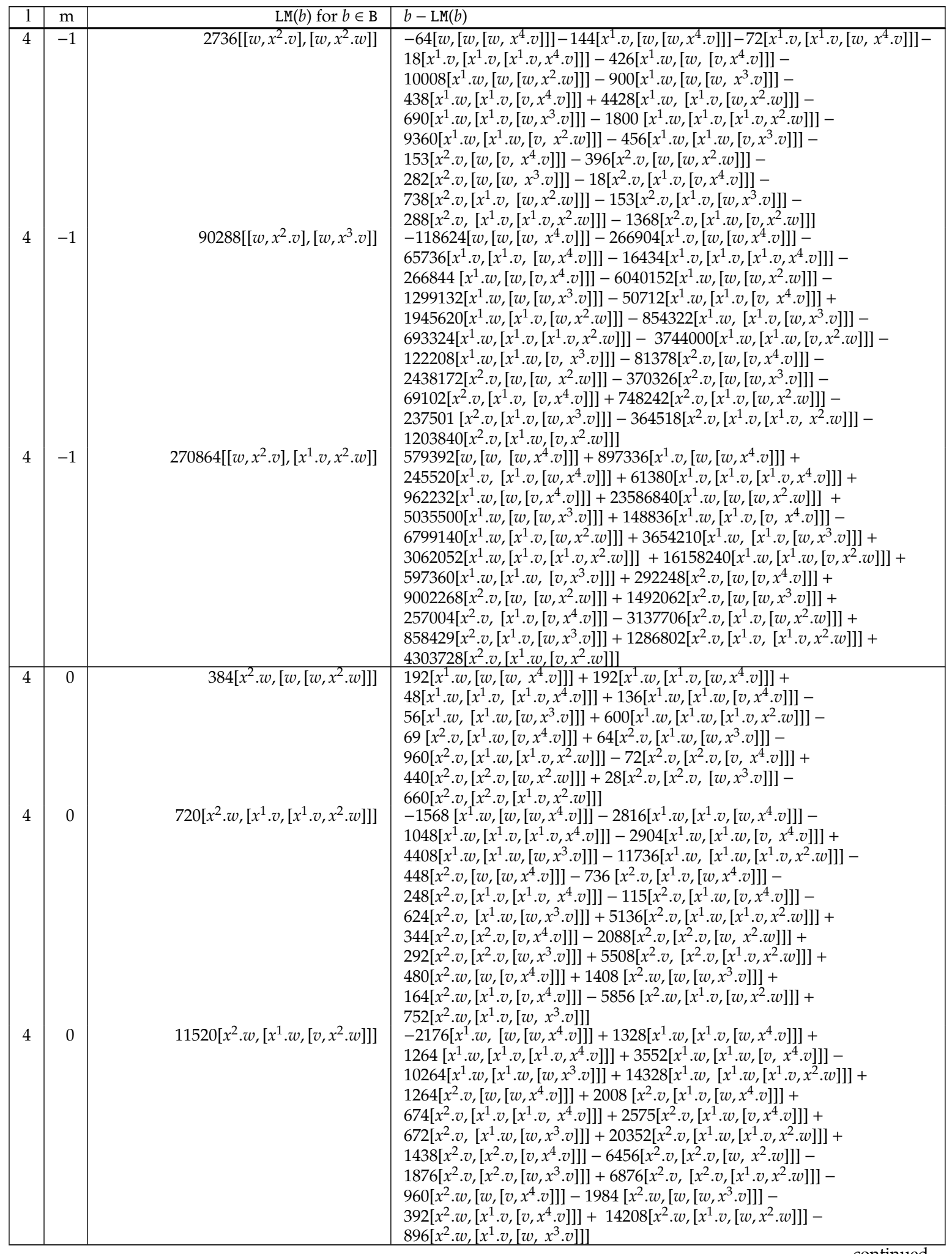


Appendix D. A Pseudo-Hall basis of $\mathfrak{g}$ for $d=3$

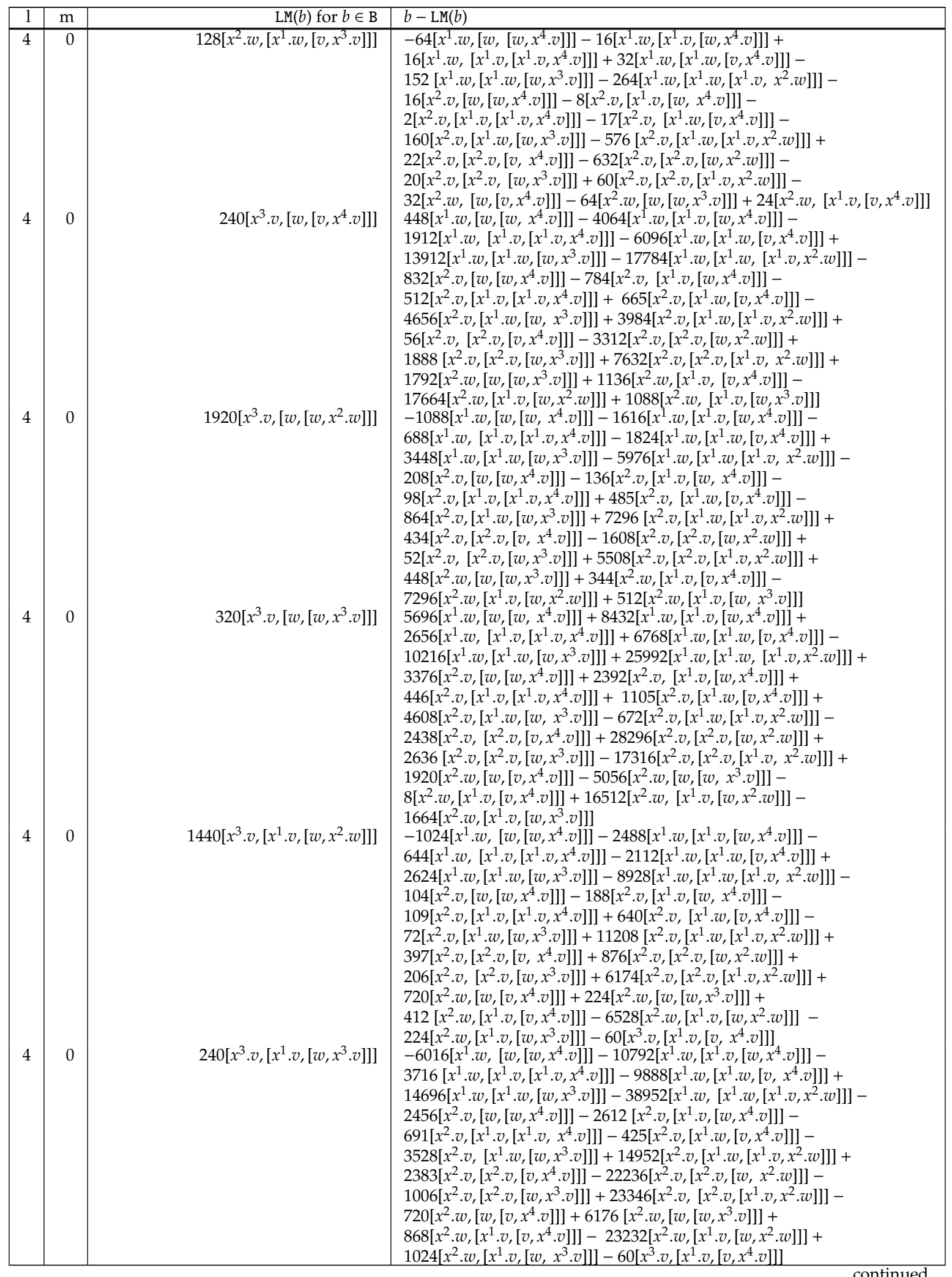




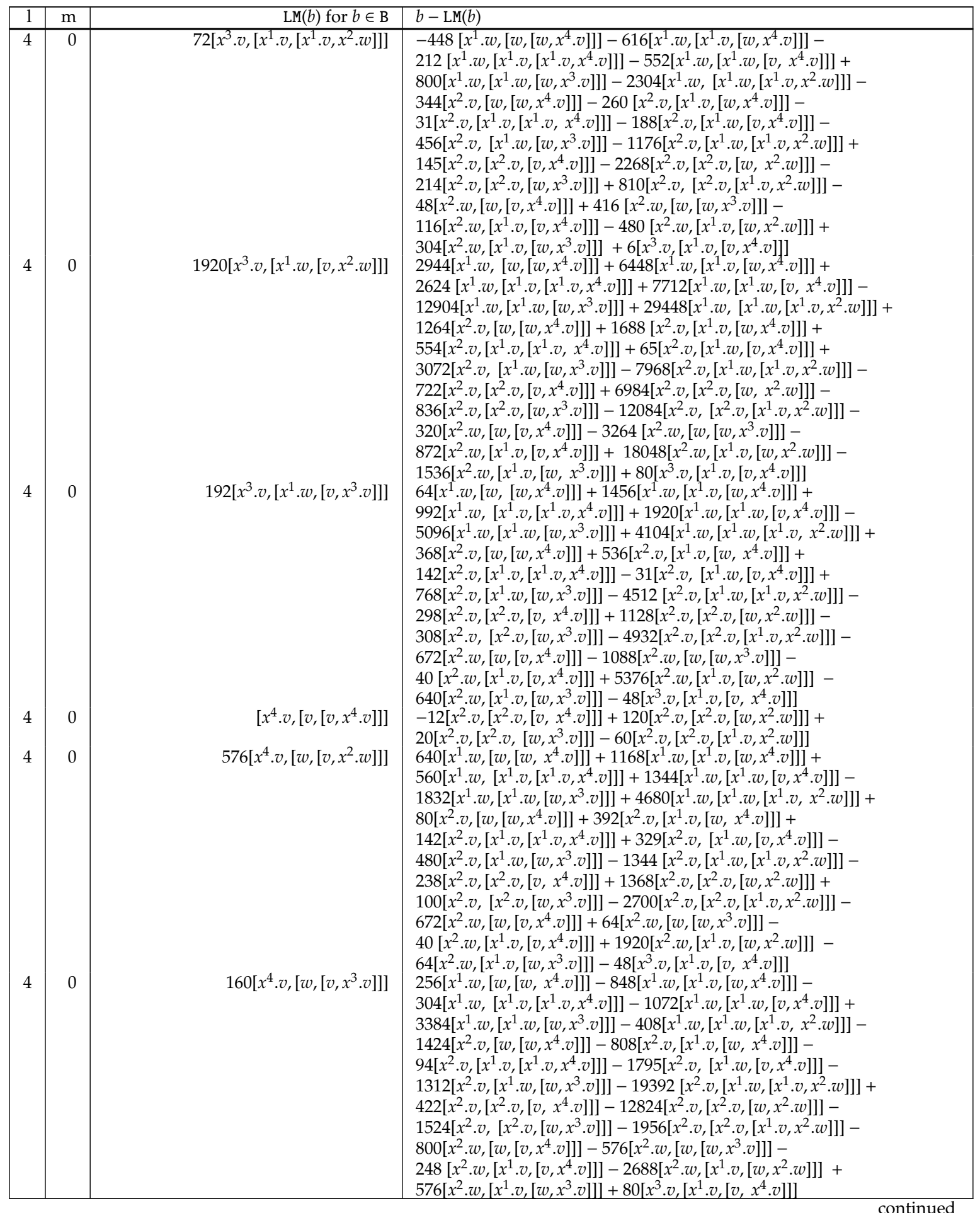


Appendix D. A Pseudo-Hall basis of $\mathfrak{g}$ for $d=3$

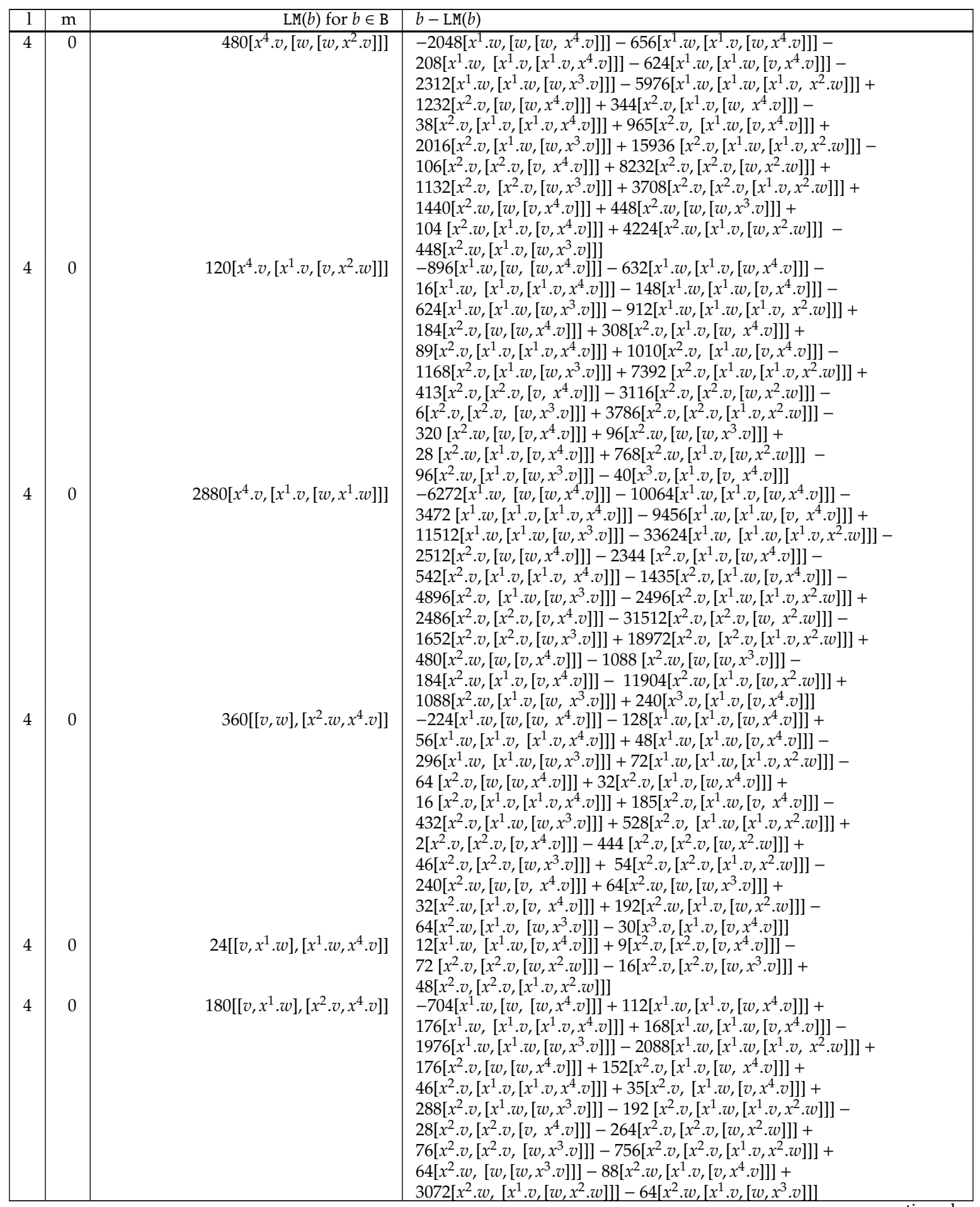

continued 


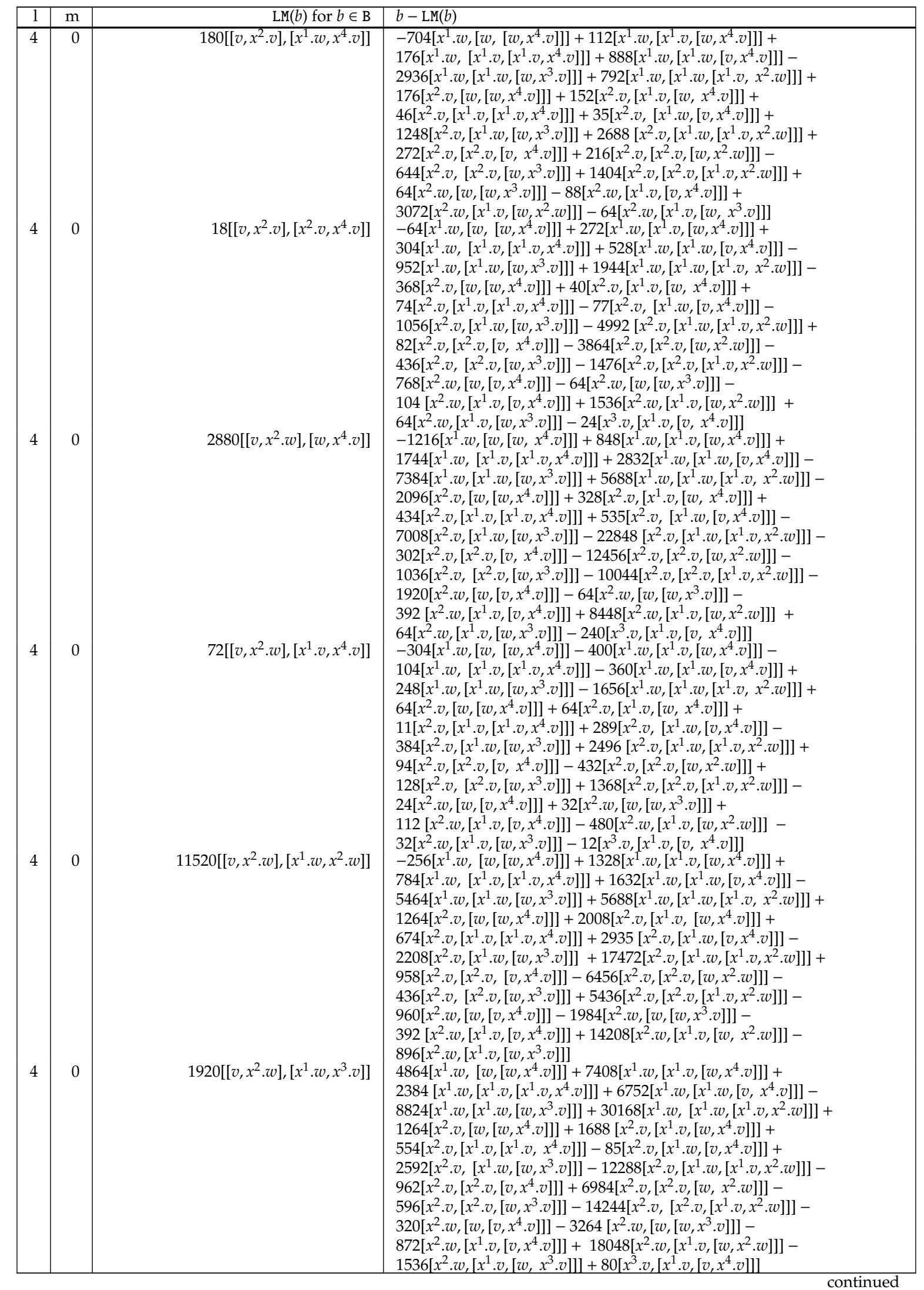


Appendix D. A Pseudo-Hall basis of $\mathfrak{g}$ for $d=3$

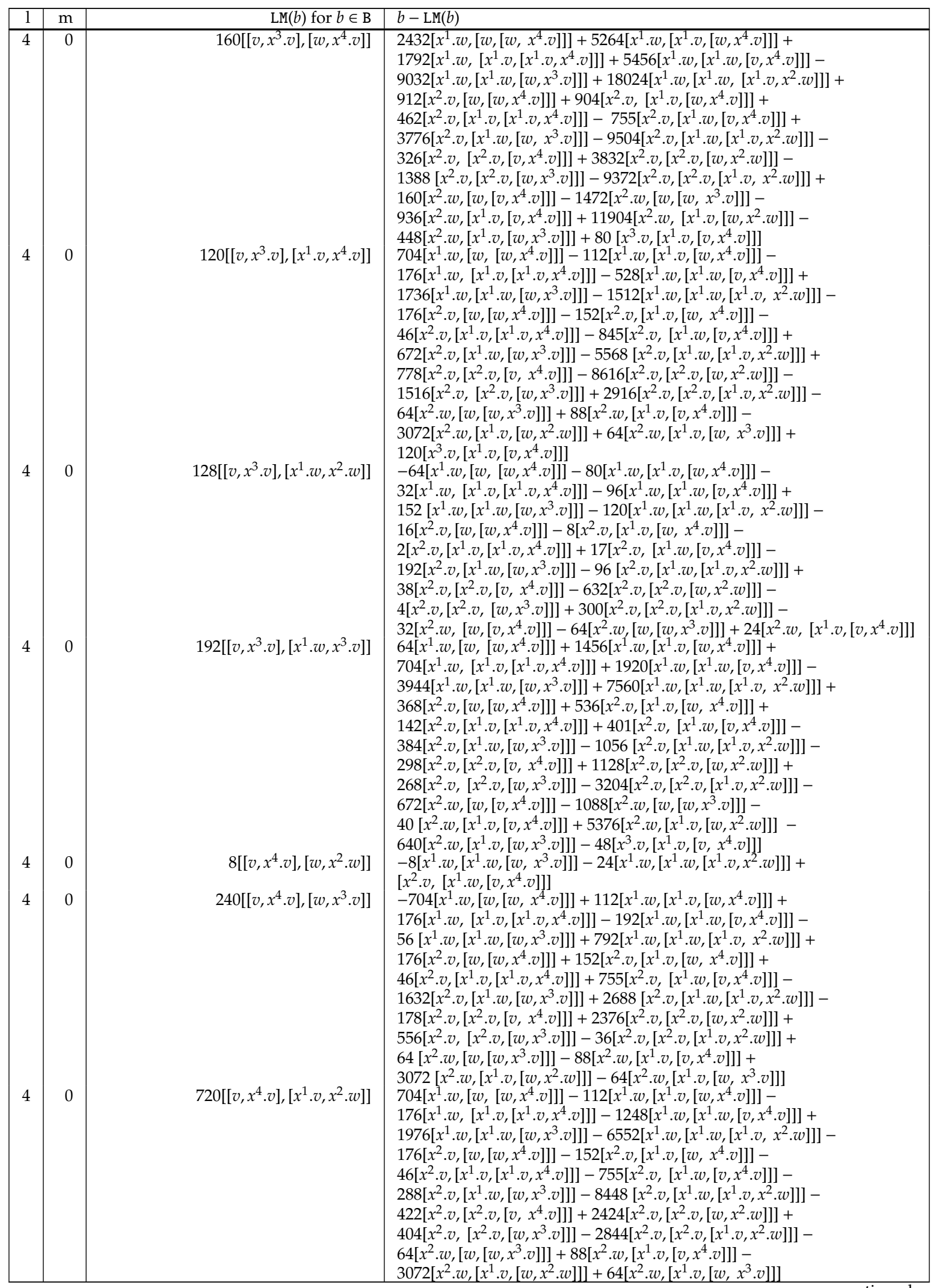




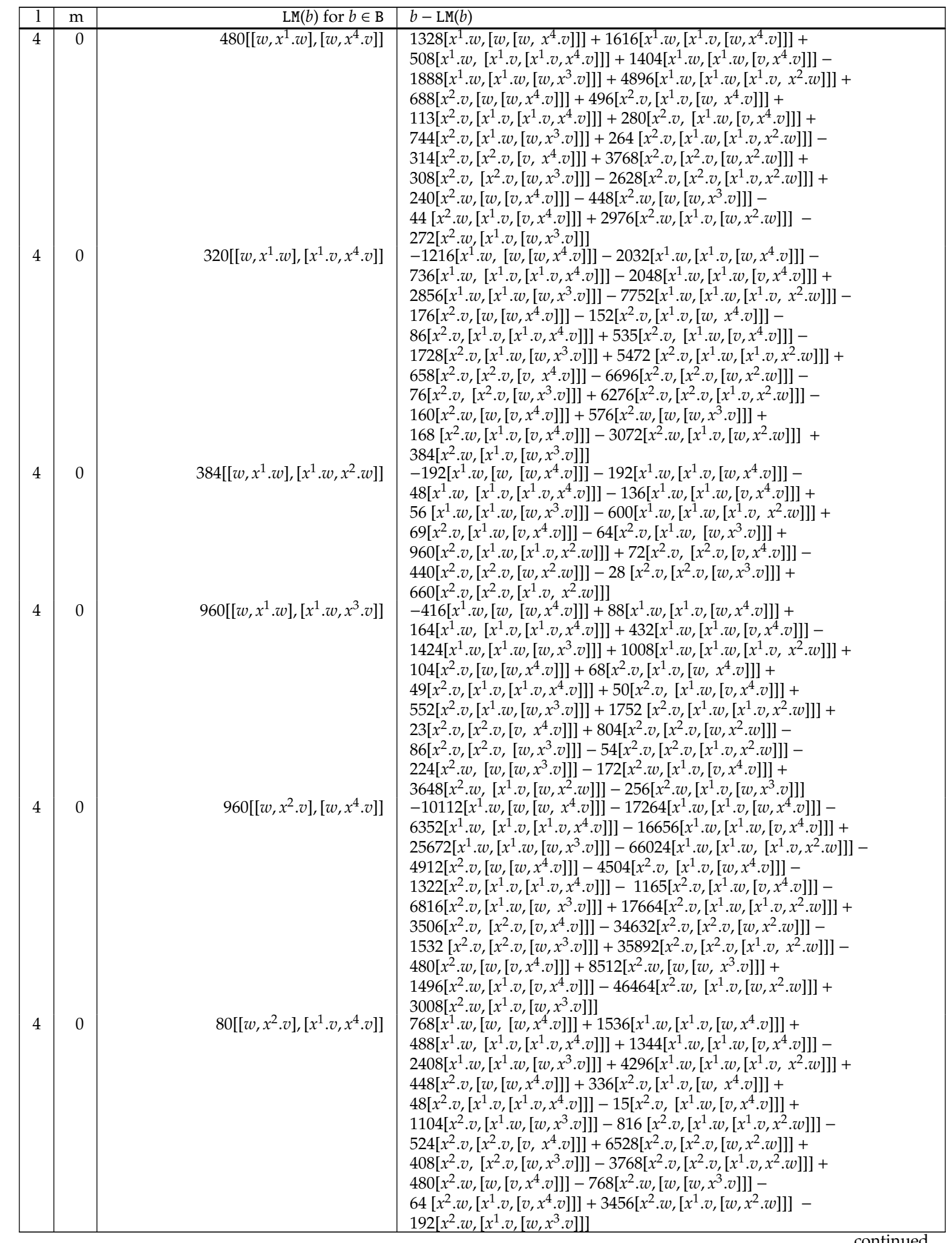


Appendix D. A Pseudo-Hall basis of $\mathfrak{g}$ for $d=3$

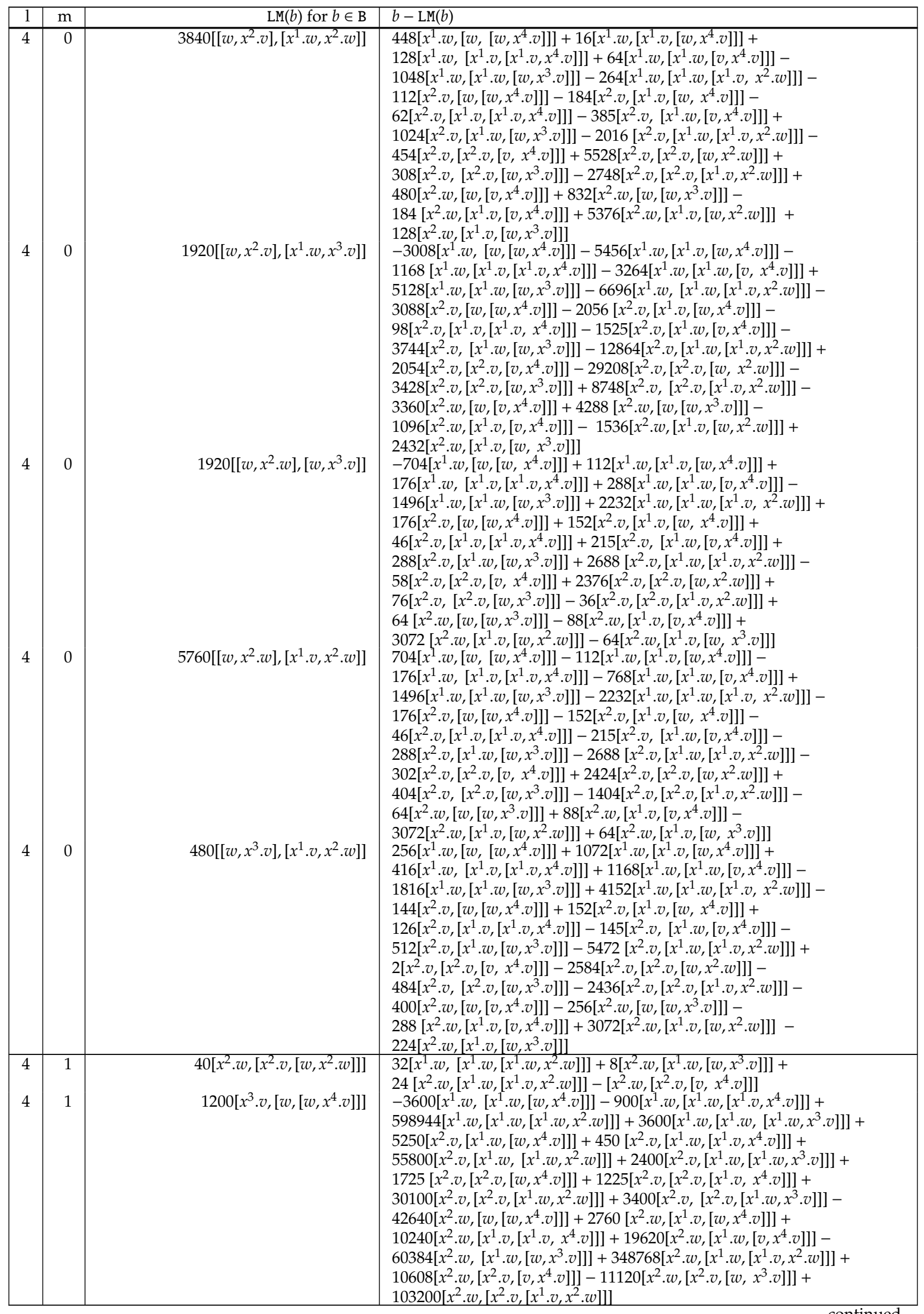




\begin{tabular}{|c|c|c|c|}
\hline 1 & $\mathrm{~m}$ & $\mathrm{LM}(b)$ for $b \in \mathrm{B}$ & $b-\mathrm{LM}(b)$ \\
\hline 4 & 1 & $600\left[x^{3} . v,\left[x^{1} . v,\left[w, x^{4} . v\right]\right]\right]$ & 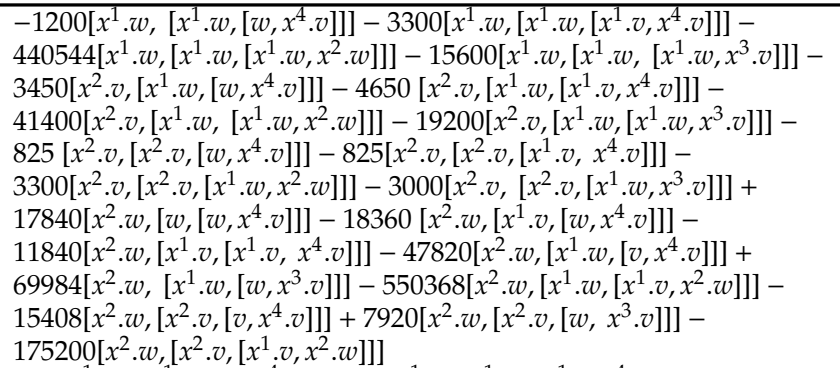 \\
\hline 4 & 1 & $6\left[x^{3} . v,\left[x^{1} . v,\left[x^{1} . v, x^{4} . v\right]\right]\right]$ & 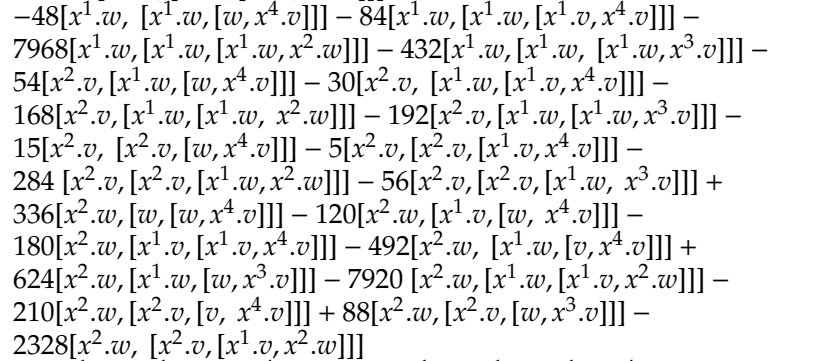 \\
\hline 4 & 1 & $75\left[x^{3} . v,\left[x^{1} . w,\left[v, x^{4} . v\right]\right]\right]$ & 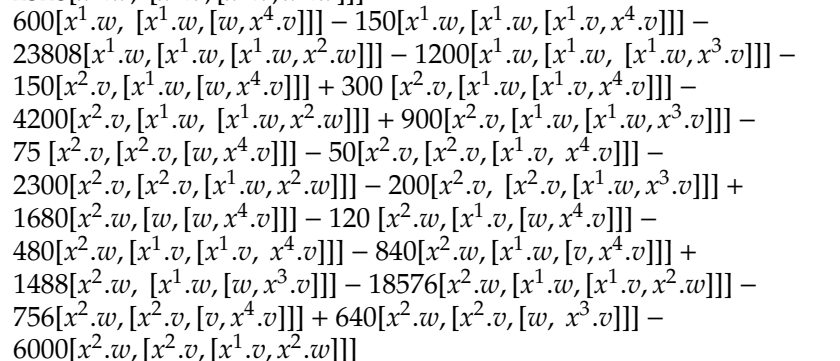 \\
\hline 4 & 1 & $2400\left[x^{3} . v,\left[x^{1} . w,\left[w, x^{3} . v\right]\right]\right]$ & 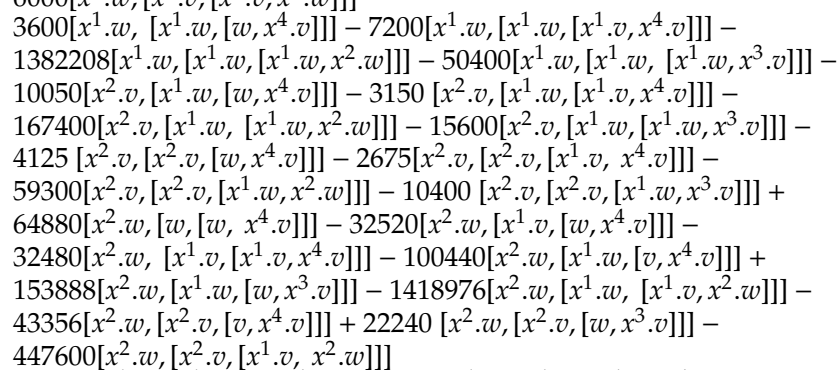 \\
\hline 4 & 1 & $3600\left[x^{3} \cdot v,\left[x^{1} \cdot w,\left[x^{1} \cdot v, x^{2} . w\right]\right]\right]$ & 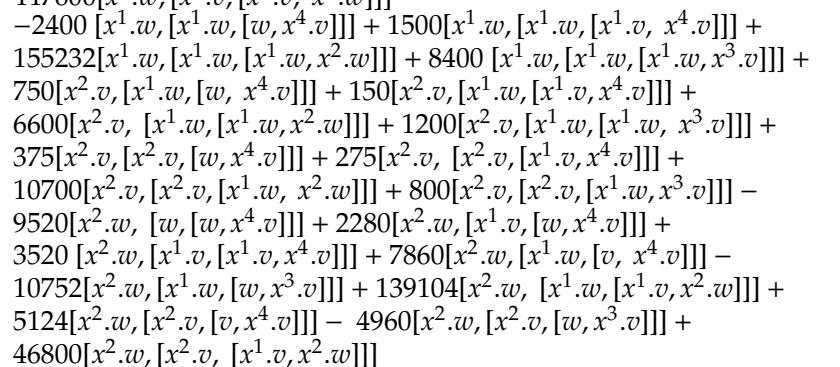 \\
\hline 4 & 1 & $100\left[x^{3} . v,\left[x^{2} . v,\left[v, x^{4} . v\right]\right]\right]$ & 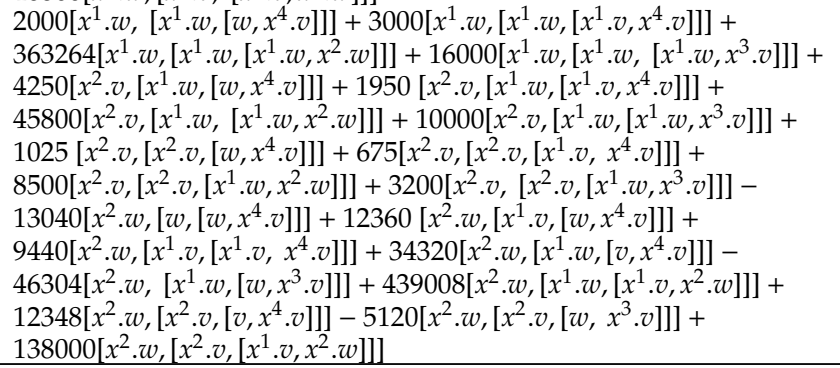 \\
\hline
\end{tabular}


Appendix D. A Pseudo-Hall basis of $\mathfrak{g}$ for $d=3$

\begin{tabular}{|c|c|c|c|}
\hline 1 & $\mathrm{~m}$ & $\mathrm{LM}(b)$ for $b \in \mathrm{B}$ & $b-\operatorname{LM}(b)$ \\
\hline 4 & 1 & $480\left[x^{3} \cdot v,\left[x^{2} \cdot v,\left[w, x^{2} . w\right]\right]\right]$ & 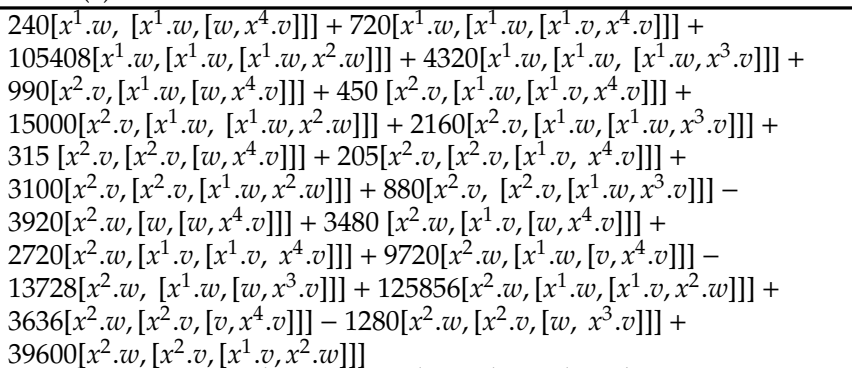 \\
\hline 4 & 1 & $150\left[x^{3} . v,\left[x^{2} . v,\left[w, x^{3} . v\right]\right]\right]$ & $\begin{array}{l}900\left[x^{1} \cdot w,\left[x^{1} \cdot w,\left[w, x^{4} \cdot v\right]\right]\right]+450\left[x^{1} \cdot w,\left[x^{1} \cdot w,\left[x^{1} \cdot v, x^{4} \cdot v\right]\right]\right]+ \\
20544\left[x^{1} \cdot w,\left[x^{1} \cdot w,\left[x^{1} \cdot v, x^{2} \cdot w\right]\right]\right]+1800\left[x^{1} \cdot w,\left[x^{1} \cdot w,\left[x^{1} \cdot w, x^{3} \cdot v\right]\right]\right]+ \\
600\left[x^{2} \cdot v,\left[x^{1} \cdot w,\left[w, x^{4} \cdot v\right]\right]\right]+450\left[x^{2} \cdot v,\left[x^{1} \cdot w,\left[x^{1} \cdot v, x^{4} \cdot v\right]\right]\right]+ \\
7200\left[x^{2} \cdot v,\left[x^{1} \cdot w,\left[x^{1} \cdot v, x^{2} \cdot w\right]\right]\right]+2100\left[x^{2} \cdot v,\left[x^{1} \cdot w,\left[x^{1} \cdot v, x^{3} \cdot v\right]\right]\right]+ \\
75\left[x^{2} \cdot v,\left[x^{2} \cdot v,\left[w, x^{4} \cdot v\right]\right]\right]+50\left[x^{2} \cdot v,\left[x^{2} \cdot v,\left[x^{1} \cdot v, x^{4} \cdot v\right]\right]\right]- \\
1600\left[x^{2} \cdot v,\left[x^{2} \cdot v,\left[x^{1} \cdot v, x^{2} \cdot w\right]\right]\right]+350\left[x^{2} \cdot v,\left[x^{2} \cdot v,\left[x^{1} \cdot w, x^{3} \cdot v\right]\right]\right]+ \\
760\left[x^{2} \cdot w,\left[w,\left[w, x^{4} \cdot v\right]\right]\right]+2460\left[x^{2} \cdot w,\left[x^{1} \cdot v,\left[w, x^{4} \cdot v\right]\right]\right]+ \\
1040\left[x^{2} \cdot w,\left[x^{1} \cdot v,\left[x^{1} \cdot v, x^{4} \cdot v\right]\right]\right]+5670\left[x^{2} \cdot w,\left[x^{1} \cdot w,\left[v, x^{4} \cdot v\right]\right]\right]- \\
5784\left[x^{2} \cdot w,\left[x^{1} \cdot w,\left[w, x^{3} \cdot v\right]\right]\right]+55368\left[x^{2} \cdot w,\left[x^{1} \cdot v,\left[x^{1} \cdot v, x^{2} \cdot w\right]\right]\right]+ \\
1533\left[x^{2} \cdot w,\left[x^{2} \cdot v,\left[v, x^{4} \cdot v\right]\right]\right]-520\left[x^{2} \cdot w,\left[x^{2} \cdot v,\left[w, x^{3} \cdot v\right]\right]\right]+ \\
18300\left[x^{2} \cdot w,\left[x^{2} \cdot v,\left[x^{1} \cdot v, x^{2} \cdot w\right]\right]\right]\end{array}$ \\
\hline 4 & 1 & $3600\left[x^{3} . v,\left[x^{2} . v,\left[x^{1} . v, x^{2} . w\right]\right]\right]$ & 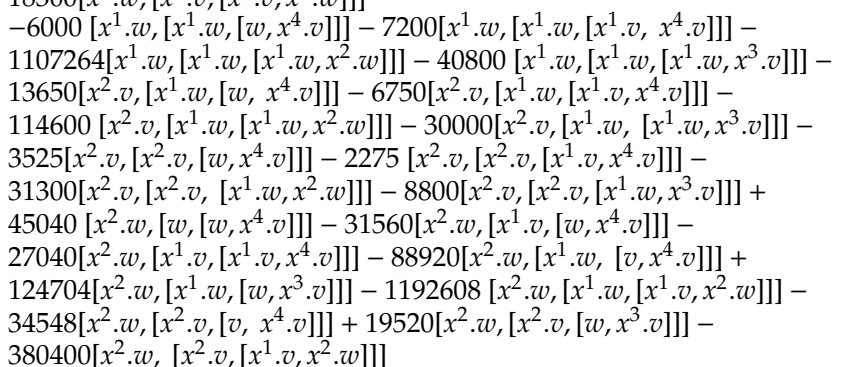 \\
\hline 4 & 1 & $150\left[x^{4} . v,\left[w,\left[v, x^{4} . v\right]\right]\right]$ & 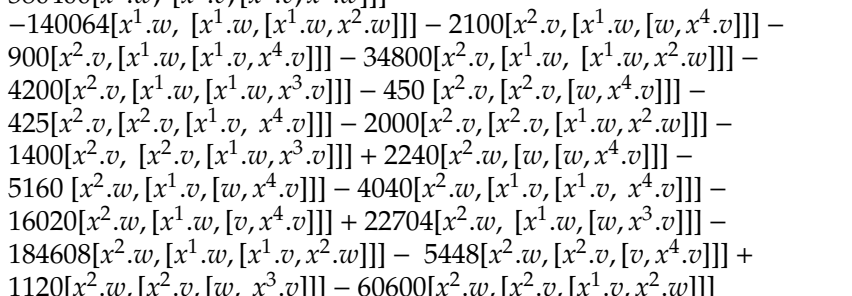 \\
\hline 4 & 1 & $28800\left[x^{4} . v,\left[w,\left[w, x^{2} . w\right]\right]\right]$ & 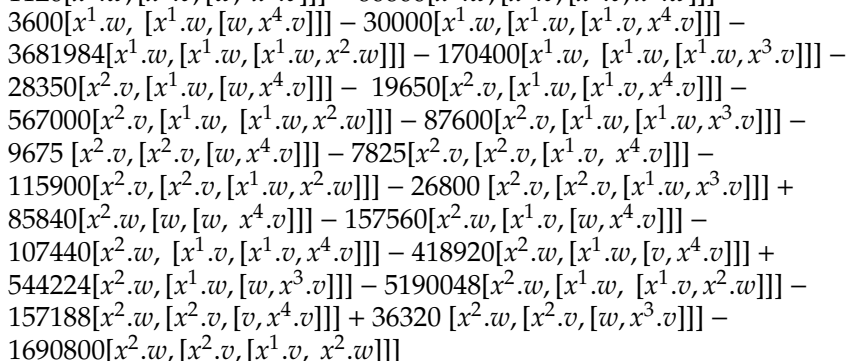 \\
\hline 4 & 1 & $2400\left[x^{4} . v,\left[w,\left[w, x^{3} . v\right]\right]\right]$ & 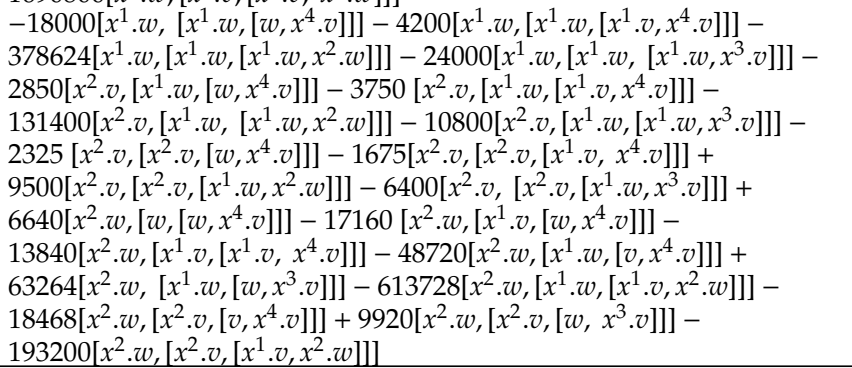 \\
\hline
\end{tabular}




\begin{tabular}{|c|c|c|c|}
\hline 1 & $\mathrm{~m}$ & $\operatorname{LM}(b)$ for $b \in \mathrm{B}$ & $b-\operatorname{LM}(b)$ \\
\hline 4 & 1 & $900\left[x^{4} . v,\left[x^{1} . v,\left[v, x^{4} . v\right]\right]\right]$ & 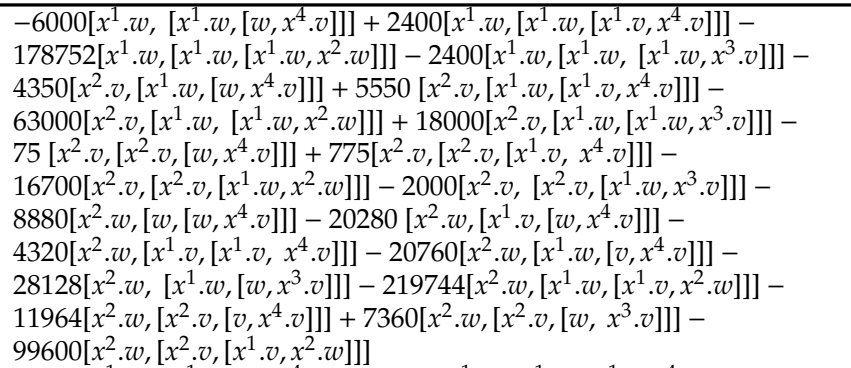 \\
\hline 4 & 1 & $8640\left[x^{4} . v,\left[x^{1} . v,\left[w, x^{2} . w\right]\right]\right]$ & 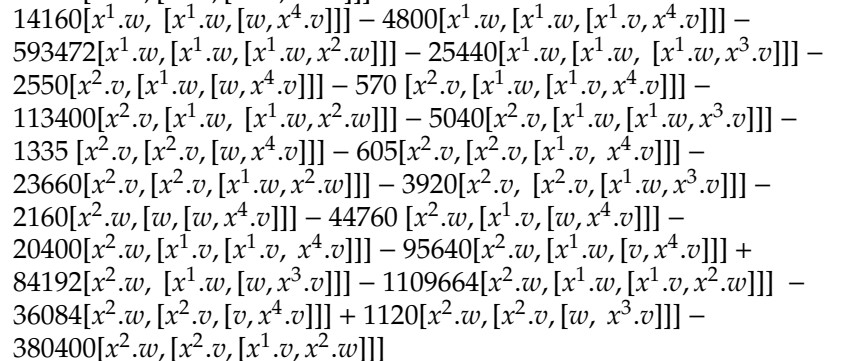 \\
\hline 4 & 1 & $900\left[x^{4} . v,\left[x^{1} . v,\left[w, x^{3} . v\right]\right]\right]$ & 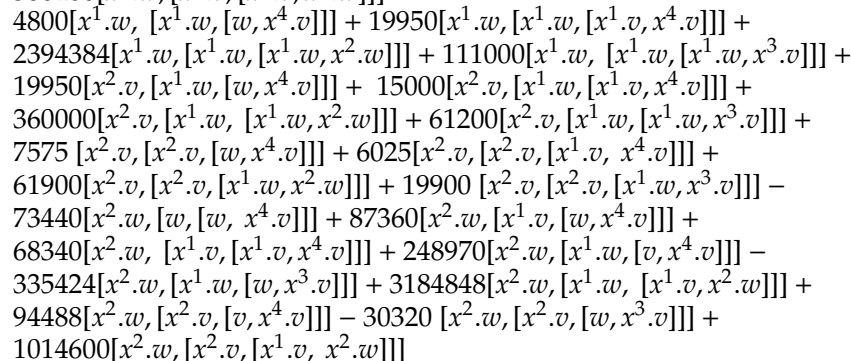 \\
\hline 4 & 1 & $21600\left[x^{4} . v,\left[x^{1} . v,\left[x^{1} . v, x^{2} . w\right]\right]\right]$ & 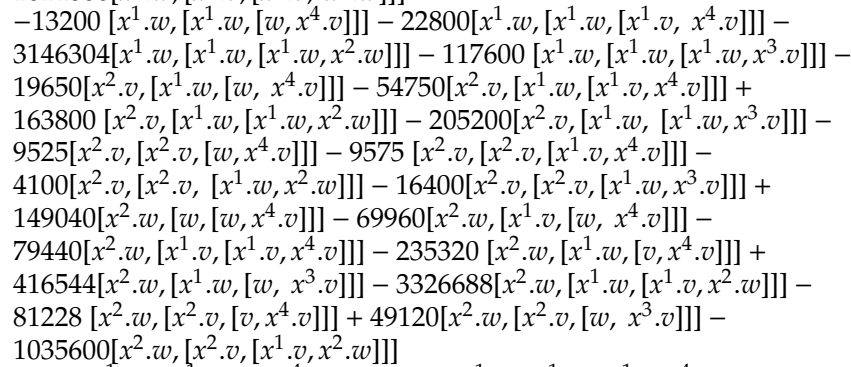 \\
\hline 4 & 1 & $43200\left[x^{4} . v,\left[x^{1} . w,\left[v, x^{2} . w\right]\right]\right]$ & 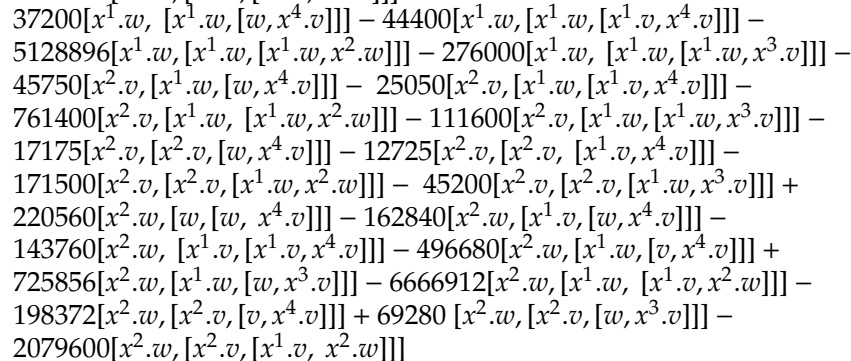 \\
\hline 4 & 1 & $1440\left[x^{4} . v,\left[x^{1} . w,\left[v, x^{3} . v\right]\right]\right]$ & 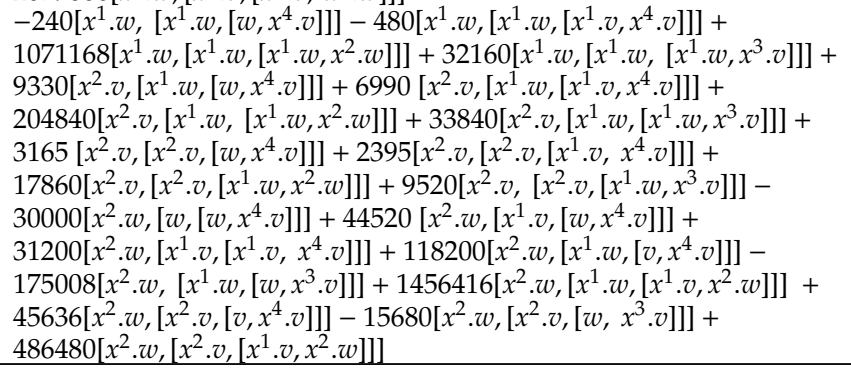 \\
\hline
\end{tabular}


Appendix D. A Pseudo-Hall basis of $\mathfrak{g}$ for $d=3$

\begin{tabular}{|c|c|c|c|}
\hline 1 & $\mathrm{~m}$ & $\operatorname{LM}(b)$ for $b \in \mathrm{B}$ & $b-\operatorname{LM}(b)$ \\
\hline 4 & 1 & $1440\left[\left[v, x^{1} . w\right],\left[x^{2} . w, x^{4} . v\right]\right]$ & 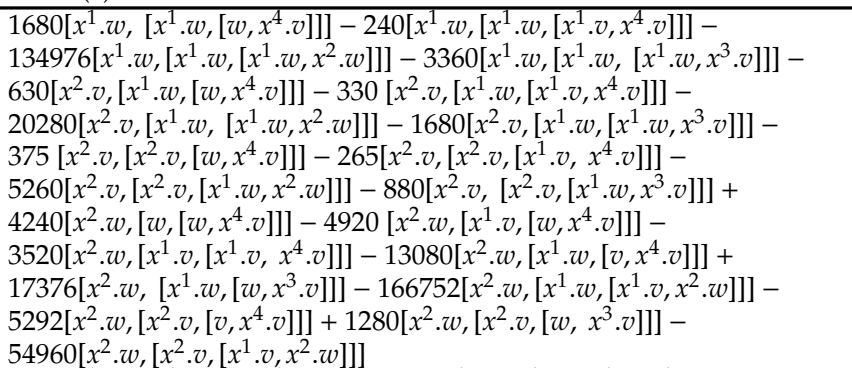 \\
\hline 4 & 1 & $5400\left[\left[v, x^{2} . v\right],\left[x^{2} . w, x^{4} . v\right]\right]$ & 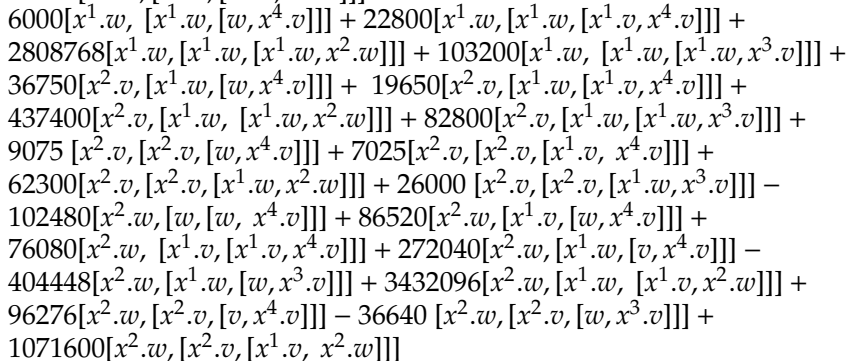 \\
\hline 4 & 1 & $1728\left[\left[v, x^{2} . w\right],\left[x^{1} . w, x^{4} . v\right]\right]$ & 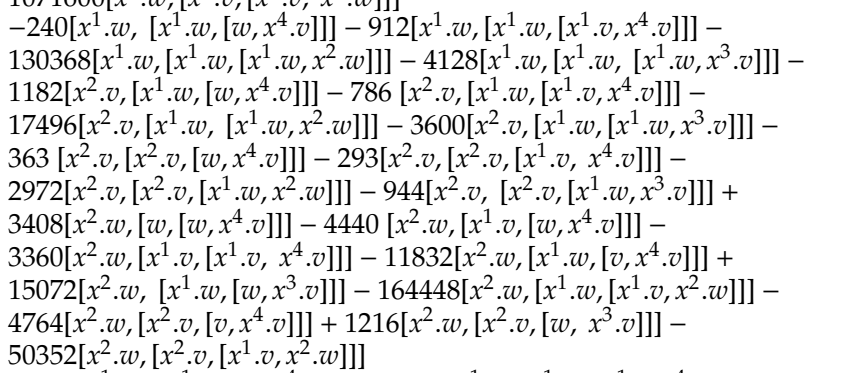 \\
\hline 4 & 1 & $10800\left[\left[v, x^{2} . w\right],\left[x^{2} . v, x^{4} . v\right]\right]$ & 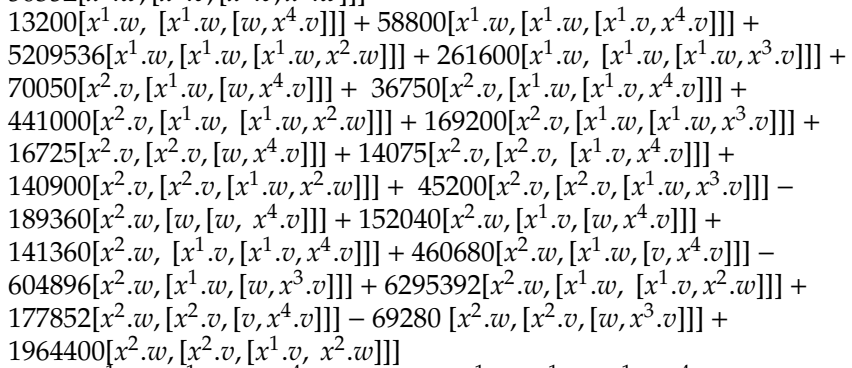 \\
\hline 4 & 1 & $7200\left[\left[v, x^{3} . v\right],\left[x^{1} . w, x^{4} . v\right]\right]$ & 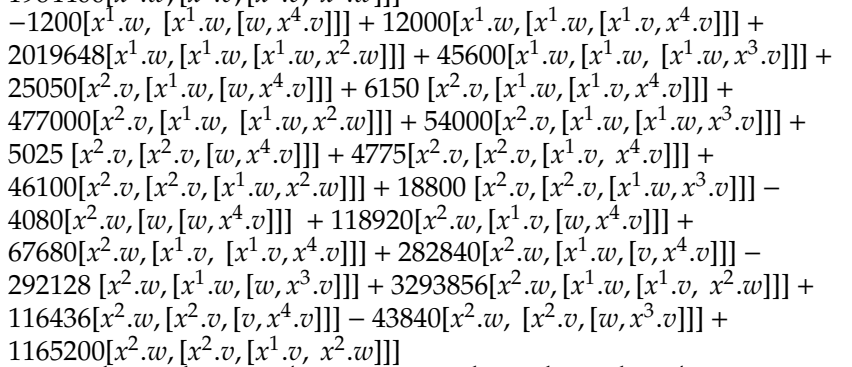 \\
\hline 4 & 1 & $1800\left[\left[v, x^{3} . v\right],\left[x^{2} . v, x^{4} . v\right]\right]$ & 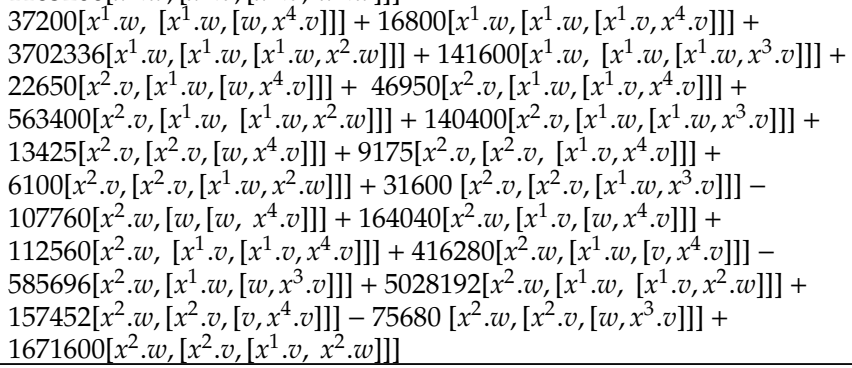 \\
\hline
\end{tabular}

continued 


\begin{tabular}{|c|c|c|c|}
\hline 1 & $\mathrm{~m}$ & $\operatorname{LM}(b)$ for $b \in \mathrm{B}$ & $b-\mathrm{LM}(b)$ \\
\hline 4 & 1 & $600\left[\left[v, x^{4} . v\right],\left[w, x^{4} . v\right]\right]$ & 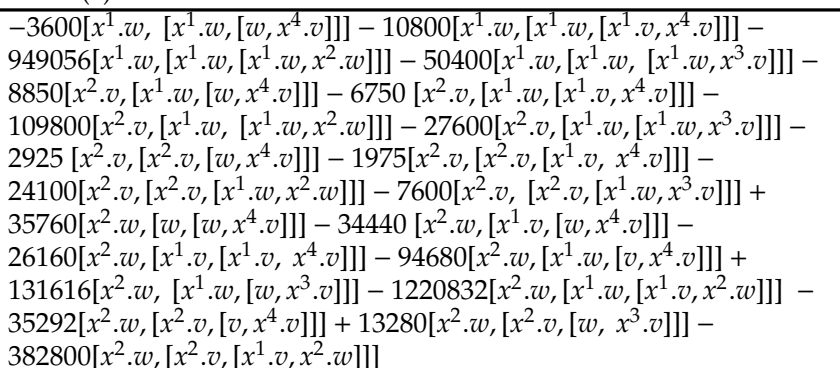 \\
\hline 4 & 1 & $75\left[\left[v, x^{4} . v\right],\left[x^{1} . v, x^{4} . v\right]\right]$ & 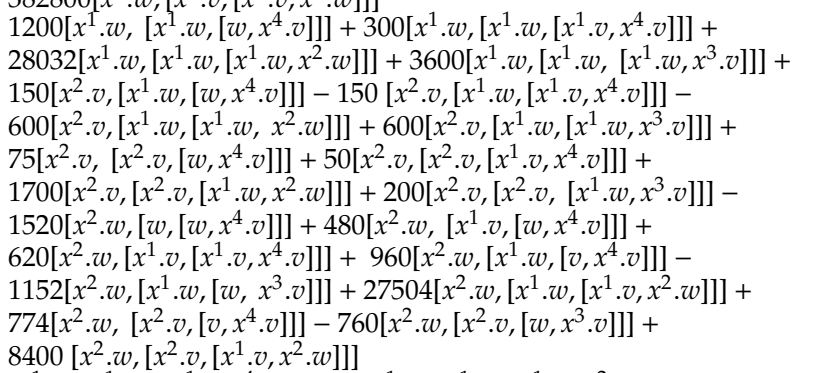 \\
\hline 4 & 1 & $2\left[\left[v, x^{4} . v\right],\left[x^{1} . w, x^{2} . w\right]\right]$ & $\begin{array}{l}{\left[x^{1} \cdot w,\left[x^{1} \cdot w,\left[x^{1} \cdot v, x^{4} \cdot v\right]\right]\right]-24\left[x^{1} \cdot w,\left[x^{1} \cdot w,\left[x^{1} \cdot w, x^{2} \cdot w\right]\right]\right]+} \\
4\left[x^{1} \cdot w,\left[x^{1} \cdot w,\left[x^{1} \cdot w, x^{3} \cdot v\right]\right]\right]-4\left[x^{2} \cdot v,\left[x^{1} \cdot w,\left[x^{1} \cdot w, x^{2} \cdot w\right]\right]\right]- \\
{\left[x^{2} \cdot w,\left[x^{1} \cdot w,\left[v, x^{4} \cdot v\right]\right]\right]}\end{array}$ \\
\hline 4 & 1 & $300\left[\left[v, x^{4} . v\right],\left[x^{1} . w, x^{3} . v\right]\right]$ & 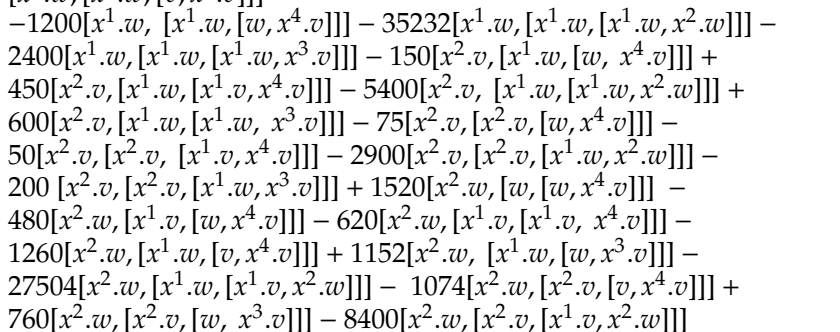 \\
\hline 4 & 1 & $28800\left[\left[w, x^{1} . w\right],\left[x^{1} . w, x^{4} . v\right]\right]$ & 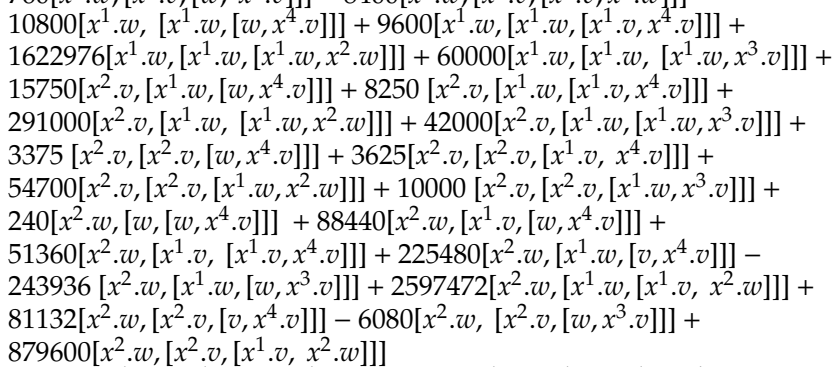 \\
\hline 4 & 1 & $7200\left[\left[w, x^{1} . w\right],\left[x^{2} . v, x^{4} . v\right]\right]$ & 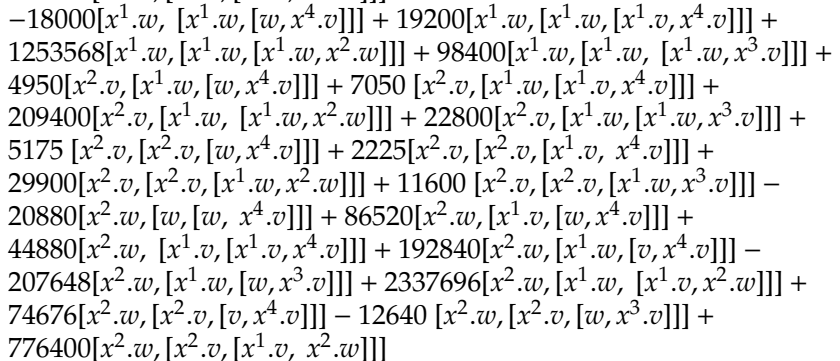 \\
\hline
\end{tabular}


Appendix D. A Pseudo-Hall basis of $\mathfrak{g}$ for $d=3$

\begin{tabular}{|c|c|c|c|}
\hline 1 & $\mathrm{~m}$ & LM $(b)$ for $b \in \mathrm{B}$ & $b-\operatorname{LM}(b)$ \\
\hline 4 & 1 & $14400\left[\left[w, x^{2} . v\right],\left[x^{1} . w, x^{4} . v\right]\right]$ & 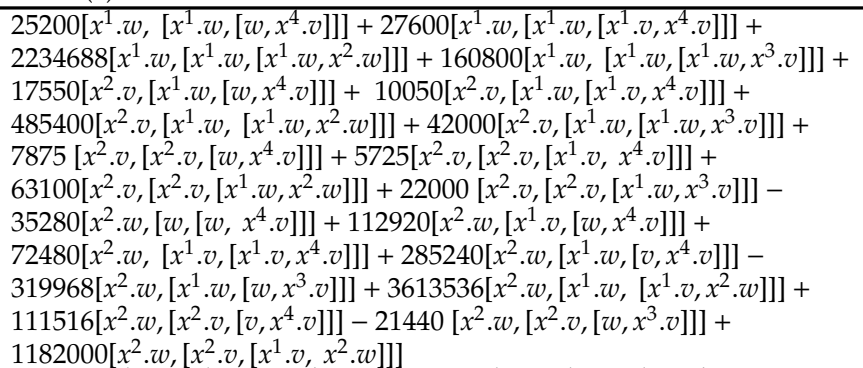 \\
\hline 4 & 1 & $3600\left[\left[w, x^{2} . v\right],\left[x^{2} . v, x^{4} . v\right]\right]$ & 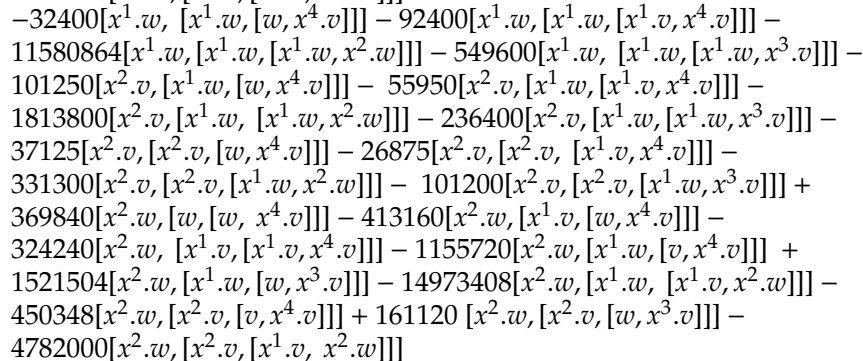 \\
\hline 4 & 1 & $4800\left[\left[w, x^{2} . w\right],\left[w, x^{4} . v\right]\right]$ & 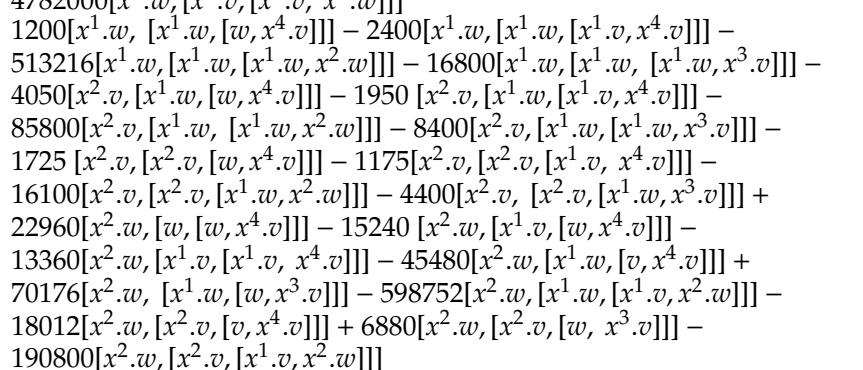 \\
\hline 4 & 1 & $600\left[\left[w, x^{2} . w\right],\left[x^{1} . v, x^{4} . v\right]\right]$ & 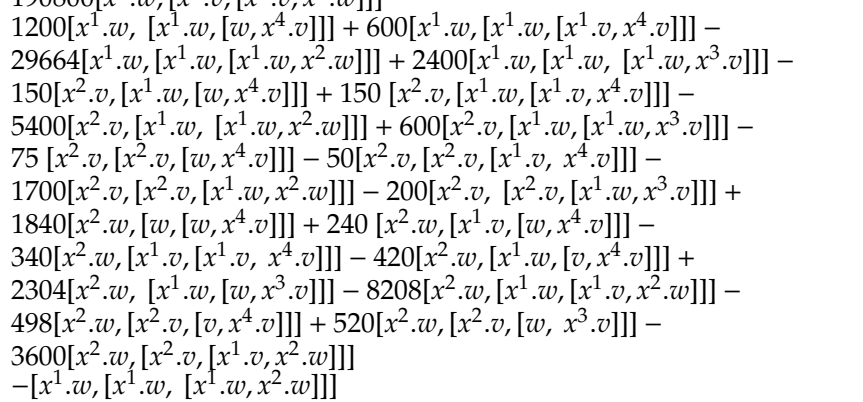 \\
\hline 4 & 1 & $2400\left[\left[w, x^{2} \cdot w\right],\left[x^{1} \cdot w, x^{3} \cdot v\right]\right]$ & 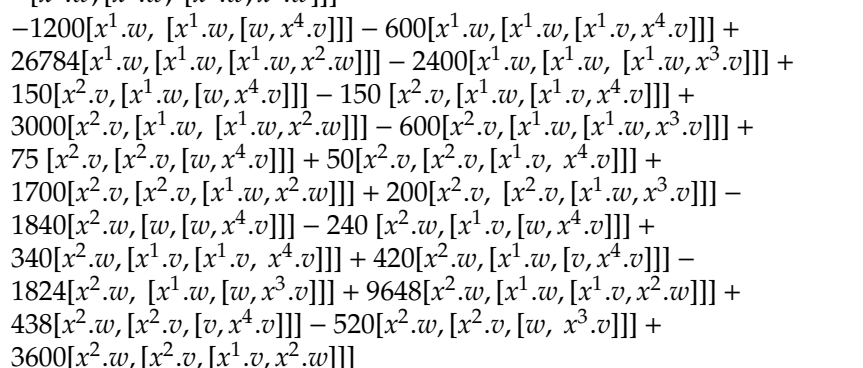 \\
\hline 4 & 1 & $2400\left[\left[w, x^{3} . v\right],\left[w, x^{4} . v\right]\right]$ & 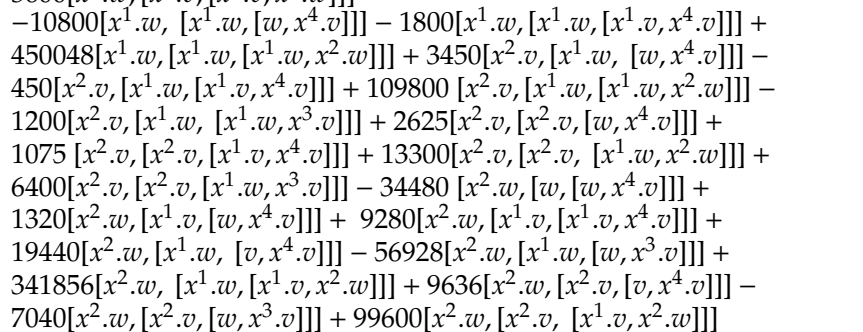 \\
\hline
\end{tabular}




\begin{tabular}{|c|c|c|c|}
\hline 1 & $\mathrm{~m}$ & $\operatorname{LM}(b)$ for $b \in \mathrm{B}$ & $b-\mathrm{LM}(b)$ \\
\hline 4 & 1 & $600\left[\left[w, x^{3} \cdot v\right],\left[x^{1} \cdot v, x^{4} \cdot v\right]\right]$ & 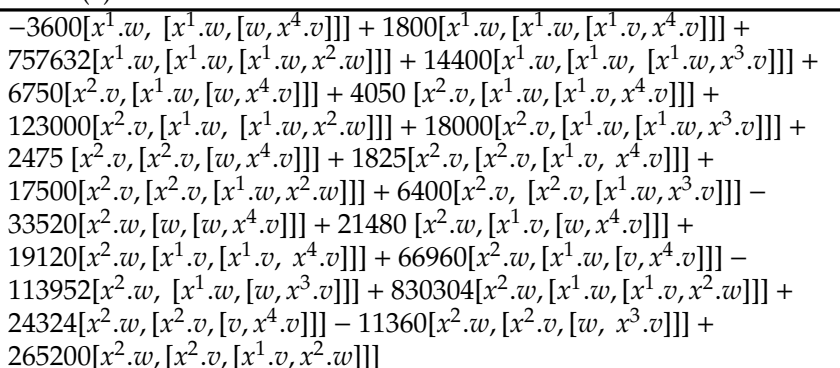 \\
\hline 4 & 1 & $300\left[\left[w, x^{3} . v\right],\left[x^{1} . w, x^{2} . w\right]\right]$ & 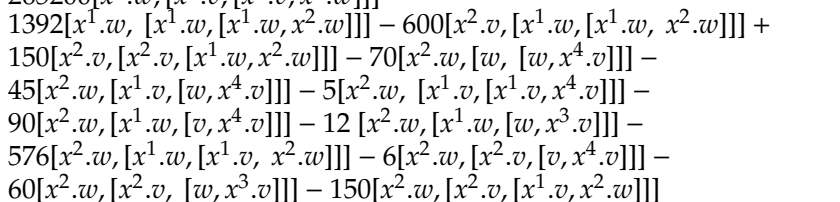 \\
\hline 4 & 1 & $2400\left[\left[w, x^{3} . v\right],\left[x^{1} . w, x^{3} . v\right]\right]$ & 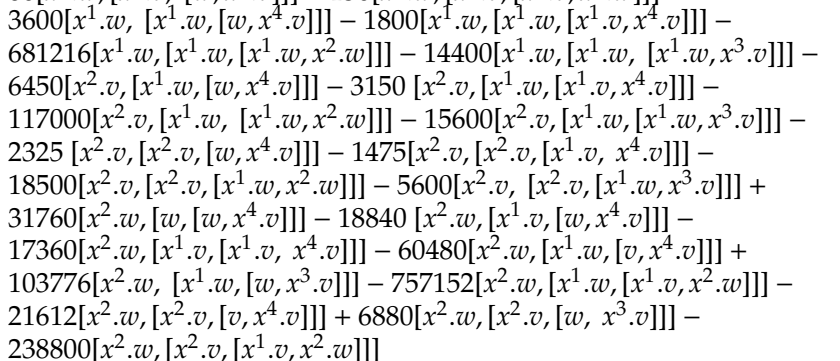 \\
\hline 4 & 1 & $7200\left[\left[w, x^{4} . v\right],\left[x^{1} . v, x^{2} . w\right]\right]$ & 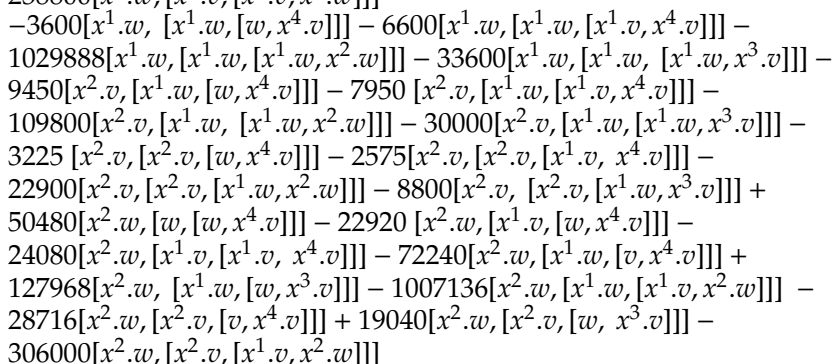 \\
\hline 4 & 1 & $900\left[\left[x^{1} . v, x^{2} \cdot w\right],\left[x^{1} \cdot v, x^{4} . v\right]\right]$ & 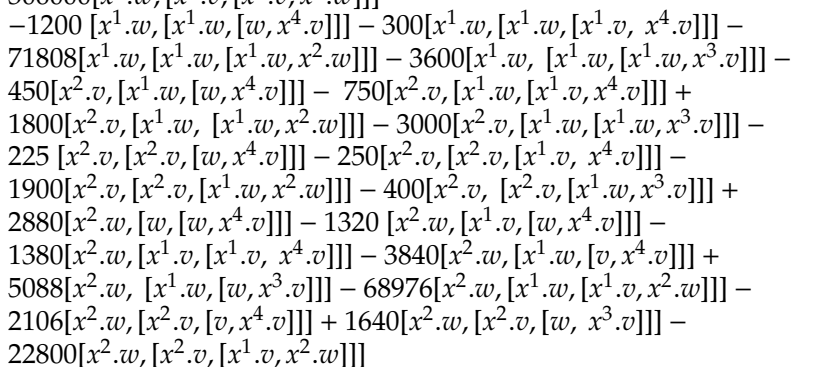 \\
\hline 4 & 1 & $60\left[\left[x^{1} . v, x^{2} . w\right],\left[x^{1} . w, x^{2} . w\right]\right]$ & 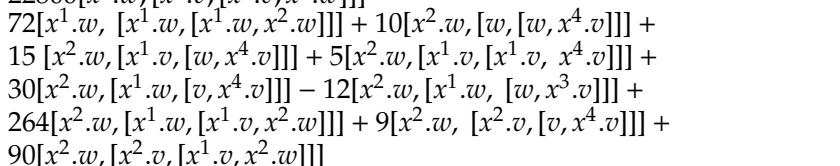 \\
\hline
\end{tabular}




\section{Appendix D. A Pseudo-Hall basis of $\mathfrak{g}$ for $d=3$}

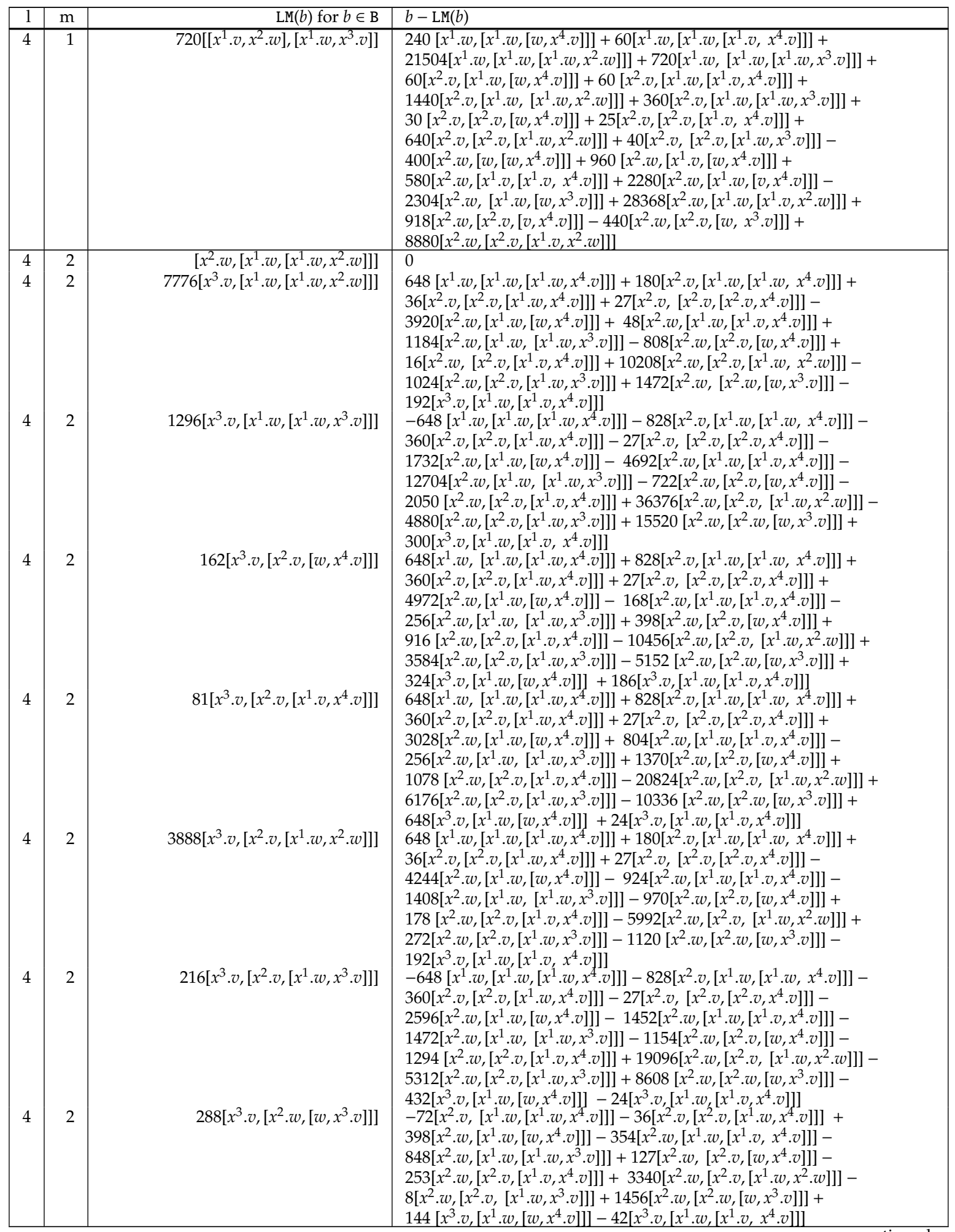




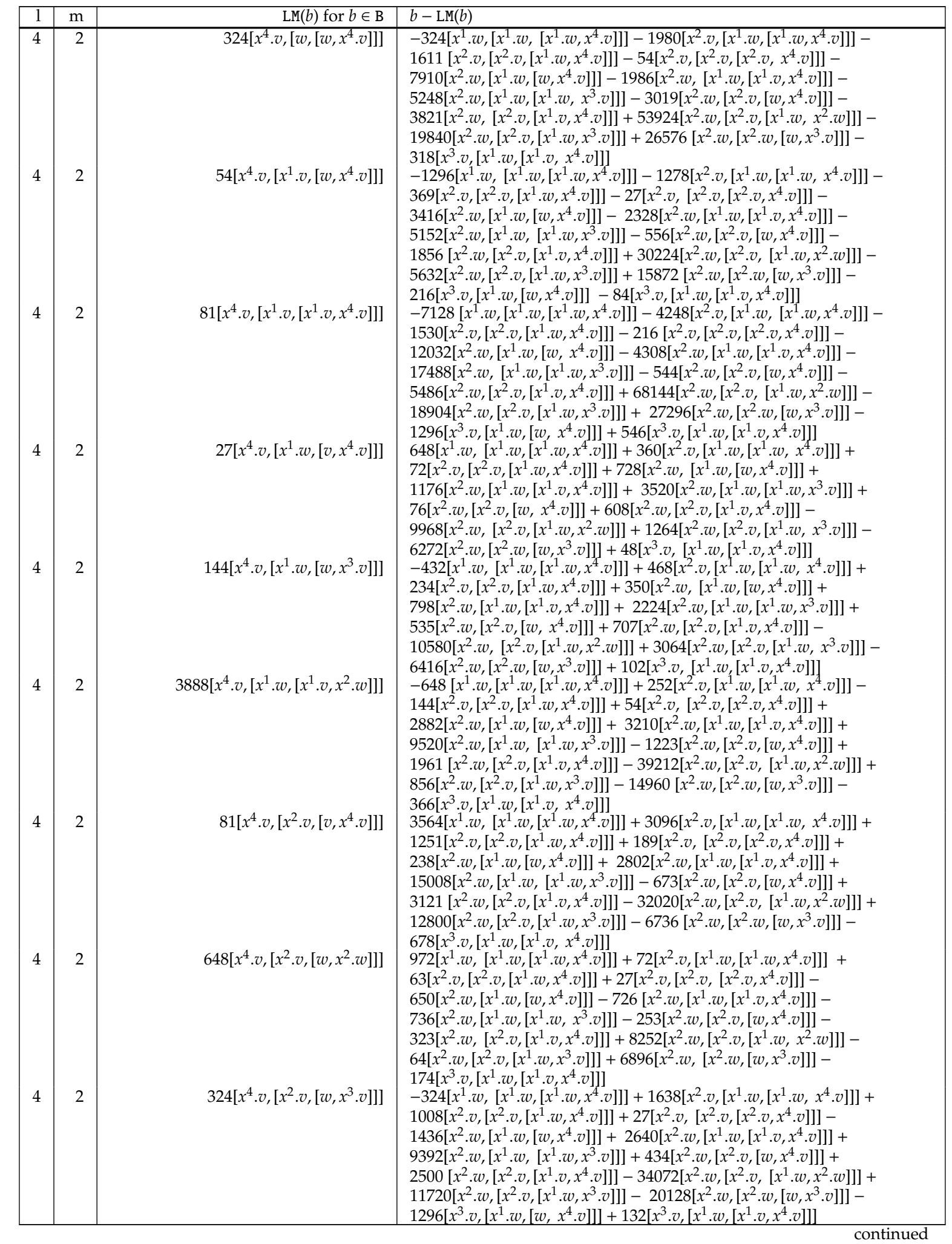


Appendix D. A Pseudo-Hall basis of $\mathfrak{g}$ for $d=3$

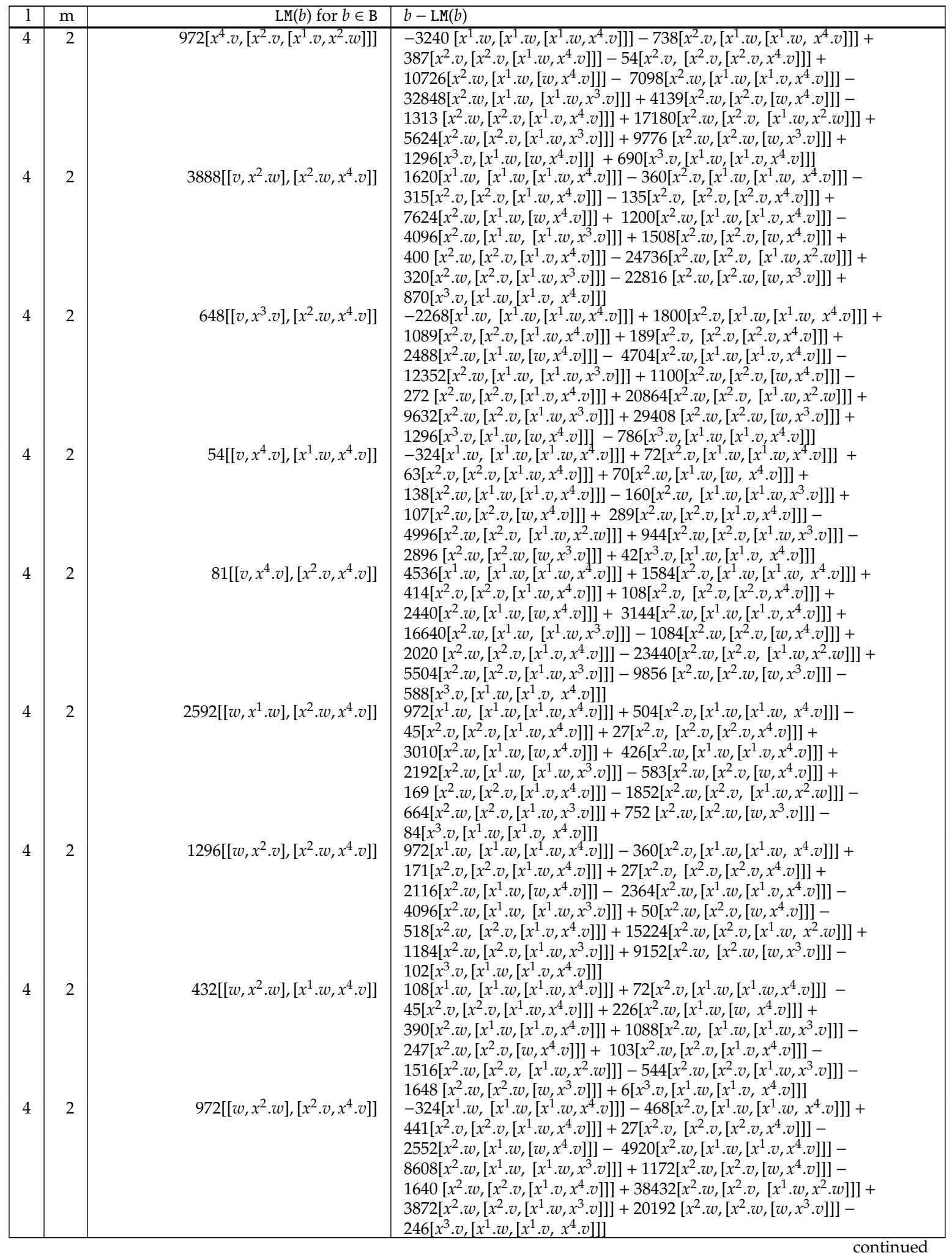




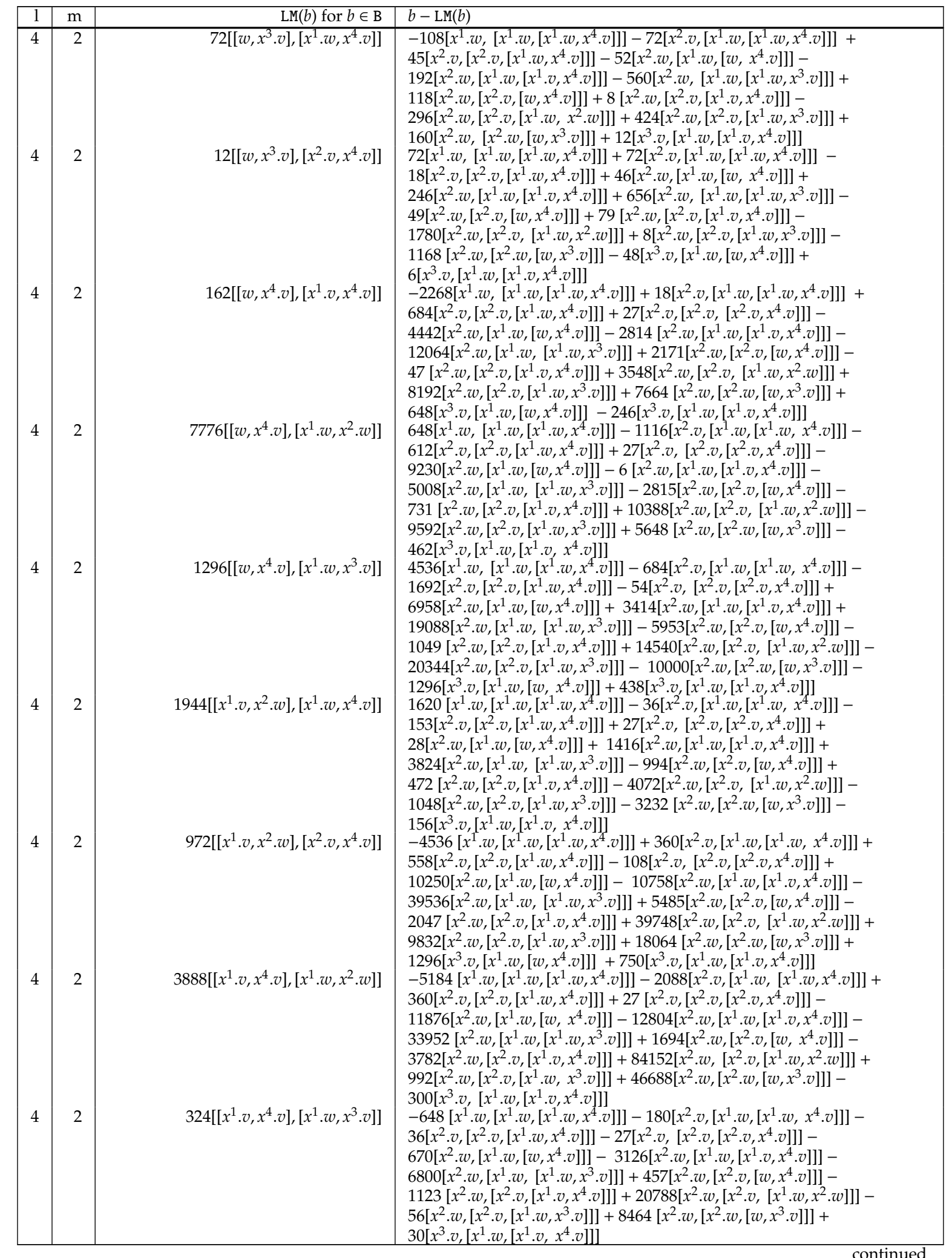


Appendix D. A Pseudo-Hall basis of $\mathfrak{g}$ for $d=3$

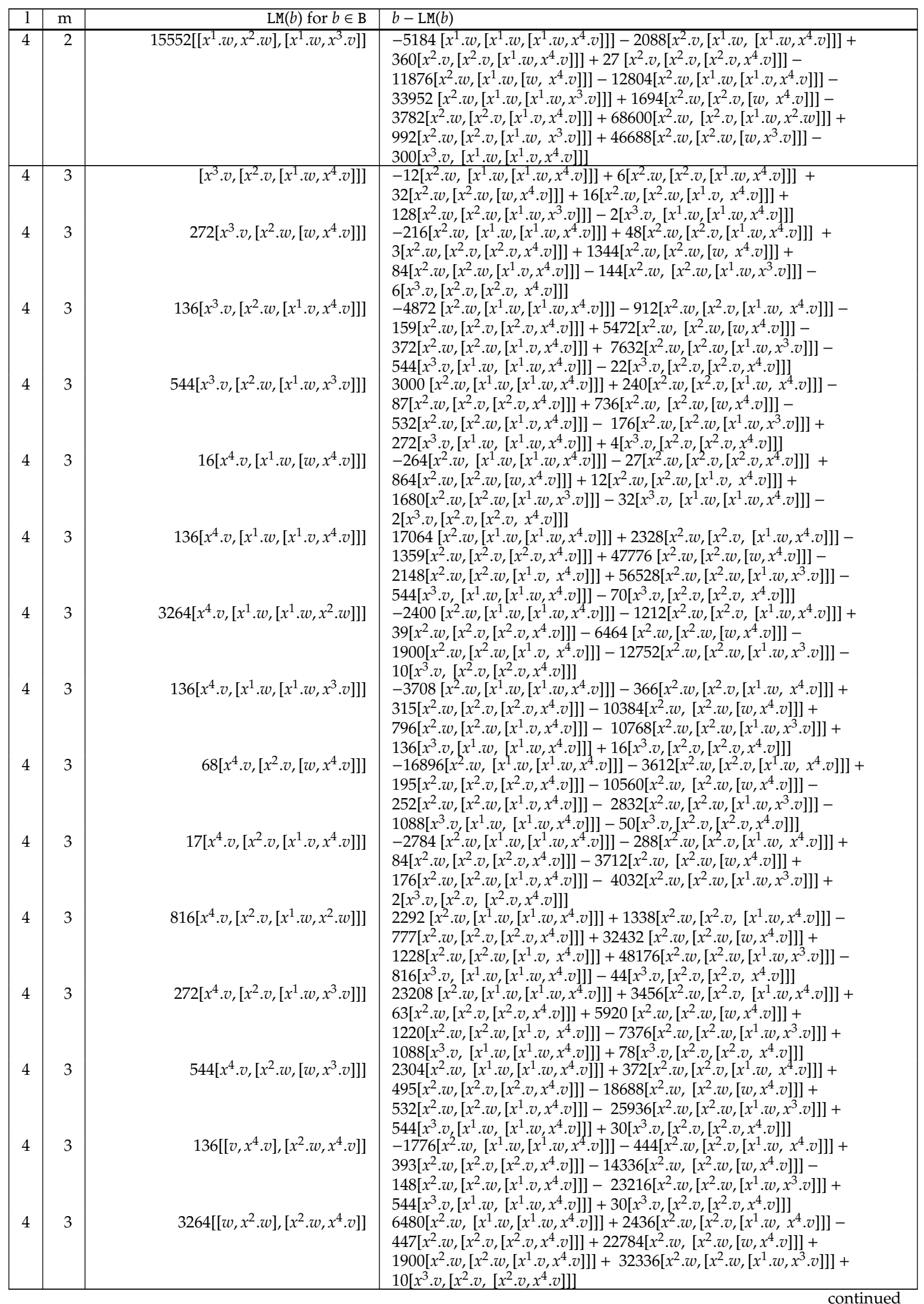




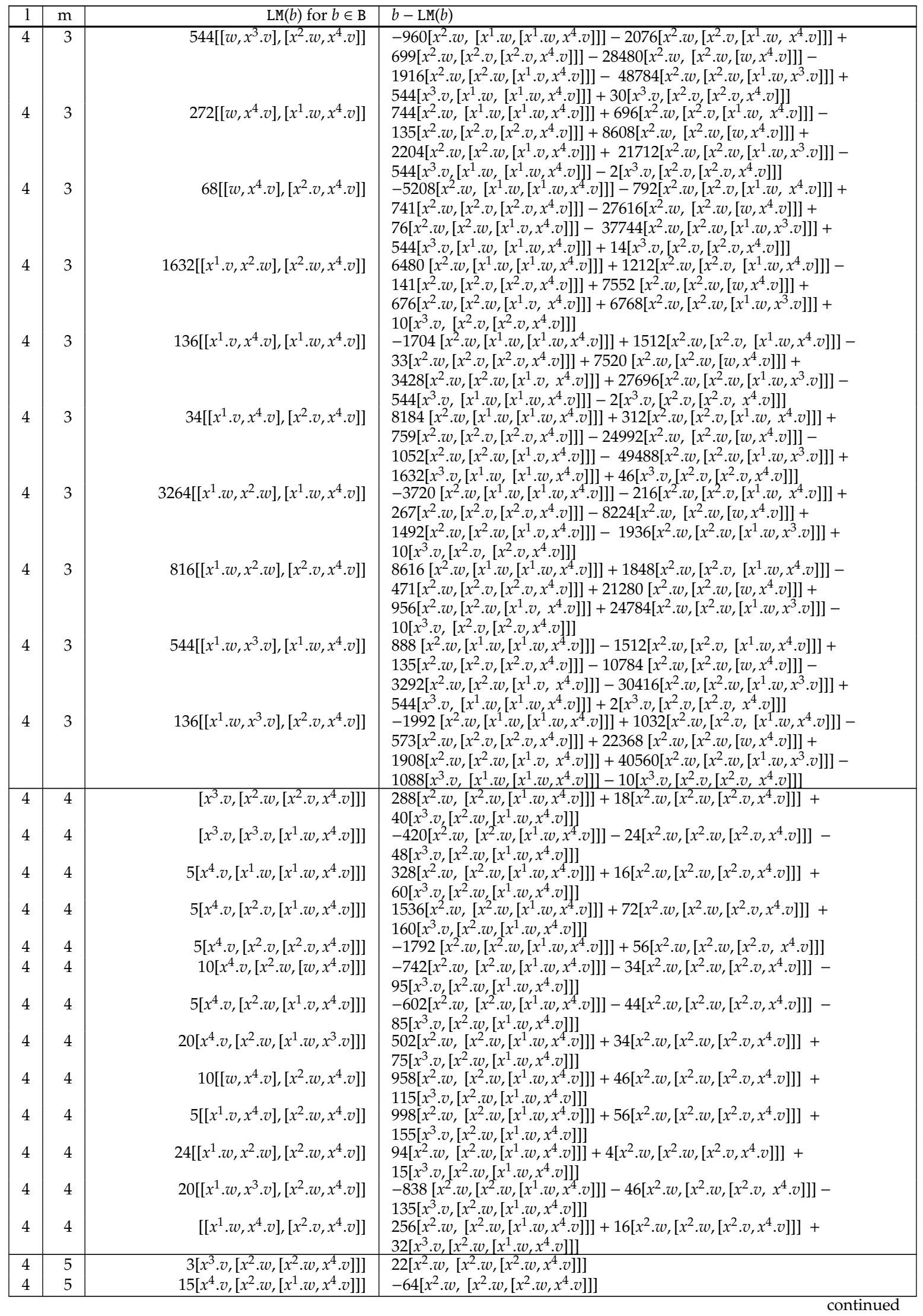


Appendix D. A Pseudo-Hall basis of $\mathfrak{g}$ for $d=3$

\begin{tabular}{|c|c|c|c|}
\hline 1 & $\mathrm{~m}$ & $\operatorname{LM}(b)$ for $b \in \mathrm{B}$ & $b-\operatorname{LM}(b)$ \\
\hline 4 & 5 & $3\left[x^{4} . v,\left[x^{2} . w,\left[x^{2} . v, x^{4} . v\right]\right]\right]$ & $112\left[x^{2} . w,\left[x^{2} . w,\left[x^{2} . w, x^{4} . v\right]\right]\right]$ \\
\hline 4 & 5 & $5\left[x^{4} . v,\left[x^{3} . v,\left[x^{1} . w, x^{4} . v\right]\right]\right]$ & $32\left[x^{2} \cdot w,\left[x^{2} \cdot w,\left[x^{2} \cdot w, x^{4} \cdot v\right]\right]\right]$ \\
\hline 4 & 5 & $3\left[\left[x^{1} . w, x^{4} . v\right],\left[x^{2} . w, x^{4} . v\right]\right]$ & $16\left[x^{2} . w,\left[x^{2} . w,\left[x^{2} . w, x^{4} . v\right]\right]\right]$ \\
\hline 4 & 5 & $15\left[\left[x^{2} . v, x^{4} . v\right],\left[x^{2} . w, x^{4} . v\right]\right]$ & $-448\left[x^{2} . w,\left[x^{2} . w,\left[x^{2} . w, x^{4} . v\right]\right]\right]$ \\
\hline 4 & 6 & {$\left[x^{4} . v,\left[x^{2} . w,\left[x^{2} . w, x^{4} . v\right]\right]\right]$} & 0 \\
\hline
\end{tabular}




\section{Bibliography}

[AL04] M. Aguiar and J.-L. Loday. Quadri-Algebras. J. Pure Appl. Algebra, 191(3):205-221, 2004.

[Ani82] D. J. Anick. Non-Commutative Graded Algebras and their Hilbert Series. J. Algebra, 78(1):120-140, 1982.

[BBS07] K. Becker, M. Becker, and J.H Schwarz. String Theory and M-Theory - A Modern Introduction. Cambridge Univ. Press, 2007.

[BM11] Dorothea Bahns and J. D. A. Meinecke. String Quantization and the Shuffle Hopf Algebra (preprint). arXiv e-prints, arXiv:1103.0974, 2011.

[dG00] W. A. de Graaf. Lie Algebras: Theory and Algorithms. Elsevier Science B.V., 1st edition, 2000.

[ES09] P. Etingof and O. Schiffmann. Lectures on Quantum Groups. Harvard University (course held 2001), 2009.

[FK15] R. Feger and T. W. Kephart. LieART - A Mathematica Application for Lie Algebras and Representation Theory. Comput. Phys. Commun., 192:166-195, 2015.

[Hal03] B. Hall. Lie Groups, Lie Algebras, and Representations. Springer, 2003.

[Han09] N. Hansen. Zur Endlichkeitshypothese der quantisierten Invariantenalgebra des Nambu-Goto-Strings. Diplomarbeit, Universität Hamburg, 2009.

[Hap93] K. M. Happle. Die Invariantenalgebra des Nambu-Goto-Strings als Poissonalgebra. Dissertation. Universität Freiburg, 1993.

[Hei90] W. Hein. Einführung in die Struktur-und Darstellungstheorie der klassischen Gruppen. Springer, 1990.

[HGK10] M Hazewinkel, N. Gubareni, and V. V. Kirichenko. Algebras, Rings and Modules. Lie Algebras and Hopf Algebras. American Mathematical Society, 1st edition, 2010.

[HPTW02] G. Handrich, C. Paufler, J. Tausk, and M. Walter. The Presentation of the Quantum Algebra of Observables of the Closed Bosonic String in 1+3 Dimensions: The Exact Quantized Generating Relations of Order 6 and 7. Mod. Phys. Lett. A, 17(40):2611-2615, 2002.

[Hum72] J. E. Humphreys. Introduction to Lie Algebras and Representation Theory. Springer, 1972. 
[Kac82] V. G. Kac. Infinite Dimensional Lie Algebras. Cambridge Univ. Press, 3rd edition, 1982.

[Kas95] C. Kassel. Quantum Groups. Springer, 1st edition, 1995.

[Lod94] J.-L. Loday. Série de Hausdorff, idempotents Eulériens et algébres de Hopf. Expo. Math., (12):165-178, 1994.

[Lov71] C. Lovelace. Pomeron Form Factors and Dual Regge Cuts. Phys. Lett. B, 34(6):500506, 1971.

[Lyn54] R. C. Lyndon. On Burnside's Problem. Trans. Am. Math. Soc., 77(2):202-215, 1954.

[Mei09] J. D. A. Meinecke. Die Invariantenalgebra des Nambu-Goto-Strings als Shuffle-HopfAlgebra und ihre Quantisierung. Diplomarbeit, Universität Göttingen, 2009.

[Mel89] Melançon, G., and Reutenauer, C. Lyndon Words, Free Algebras and Shuffles. Can. J. Math, 41(4):577-591, 1989.

[Meu01] C. Meusburger. Die Quantisierung der Invariantenalgebra des Nambu-Goto-Strings mit Hilfe einer konkreten Realisierung. Diplomarbeit. Universität Freiburg, 2001.

[MR03] C. Meusburger and K.-H. Rehren. Algebraic Quantization of the Closed Bosonic String. Commun. Math. Phys., 237(1):69-85, 2003.

[Poh82] K. Pohlmeyer. A Group-theoretical Approach to the Quantization of the Free Relativistic Closed String. Phys. Lett. B, 119 B(1-3):100-104, 1982.

[Poh85] K. Pohlmeyer. An Approach Towards the Quantization of the Relativistic Closed String Based Upon Symmetries. In N. Sanchez, editor, Non-Linear Equations in Classical and Quantum Field Theory, volume 226 of Lecture Notes in Physics, pages 159-170. Springer Berlin / Heidelberg, 1985.

[Poh89] K. Pohlmeyer. Solutions of the Constraints for Tensors Generated by Iterated Integrals. Linear Algebra Its Appl., 118:11-60, 1989.

[Poh94] K. Pohlmeyer. Uncovering the Detailed Structure of the Algebra Formed by the Invariant Charges of Closed Bosonic Strings Moving in 1+2-Dimensional Minkowski space. Commun. Math. Phys., 163(3):629-643, 1994.

[Poh99] K. Pohlmeyer. The Nambu-Goto-Theory of Closed Bosonic Strings Moving in 1+3-Dimensional Minkowski Space: The Quantum Algebra of Observables. Ann. Phys. (Berl.), 8(1):19-50, 1999.

[Poh06] K. Pohlmeyer. The Algebra of Observables of the Closed Bosonic String in (1+3)Dimensional Minkowski-Space: Updating the Structural Analysis. Mod. Phys. Lett. A, 21(23):1783-1794, 2006.

[PR86] K. Pohlmeyer and K.-H. Rehren. Algebraic Properties of the Invariant Charges of the Nambu-Goto Theory. Commun. Math. Phys., 105(4):593-627, 1986. 
[PR88] K. Pohlmeyer and K.-H. Rehren. The Invariant Charges of the Nambu-Goto Theory: Their Geometric Origin and Their Completeness. Commun. Math. Phys., 114(2):177-186, 1988.

[Rad79] D. E. Radford. A Natural Ring Basis for the Shuffle Algebra and an Application to Group Schemes. J. Algebra, 58(2):432-454, 1979.

[Reu93] C. Reutenauer. Free Lie Algebras. Oxford Science Publications, 1993.

[Ser65] J.-P. Serre. Lie Algebras and Lie Groups. Springer, 2nd edition, 1965.

[Ufn98] V. Ufnarovski. Introduction to Noncommutative Gröbner Bases Theory. London Mathematical Society Lecture Notes Series, Gröbner Bases and Applications(251):259-280, 1998.

[Wit37] E. Witt. Treue Darstellungen Liescher Ringe. J. Reine Angew. Math., 177:152-160, 1937.

[Zwi04] B. Zwiebach. A First Course in String Theory. Cambridge Univ. Press, 2004.

Lebenslauf Nils Bahne Hansen

wissenschaftliche Bildungsabschlüsse:

2008 Mathematik-Diplom, Universität Hamburg

2009 Physik-Diplom, Universität Hamburg

Stand: November 2020 